\title{
WestVirginiaUniversity
}

THE RESEARCH REPOSITORY @ WVU

Graduate Theses, Dissertations, and Problem Reports

2017

\section{Modeling, Simulation and Decentralized Control of Islanded Microgrids}

\author{
Farideh Doost Mohammadi
}

Follow this and additional works at: https://researchrepository.wvu.edu/etd

\section{Recommended Citation}

Mohammadi, Farideh Doost, "Modeling, Simulation and Decentralized Control of Islanded Microgrids" (2017). Graduate Theses, Dissertations, and Problem Reports. 7113.

https://researchrepository.wvu.edu/etd/7113

This Dissertation is protected by copyright and/or related rights. It has been brought to you by the The Research Repository @ WVU with permission from the rights-holder(s). You are free to use this Dissertation in any way that is permitted by the copyright and related rights legislation that applies to your use. For other uses you must obtain permission from the rights-holder(s) directly, unless additional rights are indicated by a Creative Commons license in the record and/ or on the work itself. This Dissertation has been accepted for inclusion in WVU Graduate Theses, Dissertations, and Problem Reports collection by an authorized administrator of The Research Repository @ WVU.

For more information, please contact researchrepository@mail.wvu.edu. 


\title{
Modeling, Simulation and Decentralized Control of Islanded Microgrids
}

\author{
Farideh Doost Mohammadi \\ Dissertation submitted to the \\ College of Engineering and Mineral Resources \\ at West Virginia University \\ in partial fulfillment of the requirements \\ for the degree of \\ Doctor of Philosophy \\ in \\ Electrical Engineering \\ Muhammad A. Choudhry, Ph.D. \\ Yaser P. Fallah, Ph.D. \\ Ali Feliachi, Ph.D., Chair \\ Powsiri Klinkhachorn, Ph.D. \\ Rong Luo, Ph.D. \\ Lane Department of Computer Science and Electrical Engineering \\ Morgantown, West Virginia \\ 2016
}

Keywords: PQ and VSI inverter, H infinity Control, Multi agent, Microgrids Control, DGs Copyright 2017 Farideh Doost Mohammadi 


\begin{abstract}
Modeling, Simulation and Decentralized Control of Islanded Microgrids Farideh Doost Mohammadi

This thesis develops a comprehensive modular state-space model of microgrids containing inverter-based Distributed Energy Resources (DERs). The model is validated and then used for small signal stability enhancement and voltage and frequency control.

State space models of various microgrid elements are first derived, which allow for the inclusion of any possible elements such as current controlled inverters that are missing in the literature. Then a complete state space model is obtained to complement the models that are available in the literature and whose objectives are system analysis only as compared to the purpose of this work which is stability enhancement and control design. Specifically,

1. Small signal stability is enhanced by adding current controlled inverters to the microgrid.

2. Decentralized secondary frequency and voltage control techniques are proposed.

For secondary frequency control purposes, at first, the control strategies of different kinds of inverters and storage devices are described. Then, a novel solution is introduced for islanded microgrids by decomposing the system into virtual control areas.

For the secondary voltage control an Average Consensus Algorithm (ACA) is used and applied on a network of agents which has been chosen optimally based on the required connectivity. The main purpose of the ACA is to keep the average voltage of all the buses at a desired level during islanding. Then another control strategy is proposed to improve the voltage profile. While the average voltage is kept fixed by the voltage controlled inverters, this voltage profile smoothness is obtained by dedicating zones to current controlled inverters and defining their responsibilities based on the location of the loads.
\end{abstract}




\section{Acknowledgements}

I bless God Almighty for His faithfulness in my life, and for the strength, sound health and sound mind $\mathrm{He}$ granted me throughout the period of the program.

I am very grateful to my supervisor and mentor Dr Ali Feliachi for his advice, support and guidance over the years. He has impacted into my life an immense deal of knowledge and without him, this work would not have been accomplished.

I also appreciate all the faculty member who have taught, encouraged and guided me in special and different ways. Special thanks go to Professor Muhammad Choudhry (WVU Lane Department of Computer Science and Electrical Engineering), Professor Yaser P. Fallah (WVU Lane Department of Computer Science and Electrical Engineering), Professor Powsiri Klinkhachorn (Lane department of Computer Science and Electrical Engineering), and Professor Rong Luo (WVU Department of Mathematics).

Lastly, I dedicate this work to my supportive husband Hessam Keshtkar and my adorable son, Nikan Keshtkar. 


\section{CONTENTS}

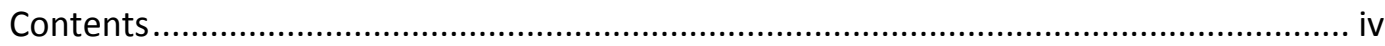

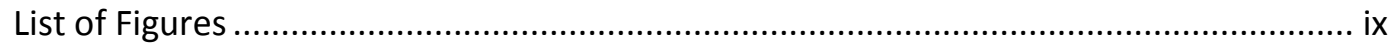

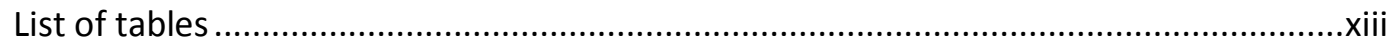

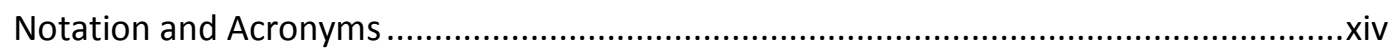

Chapter 1:

Introduction

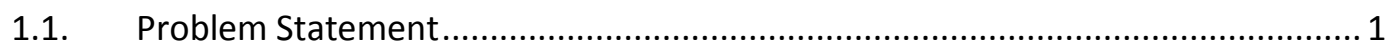

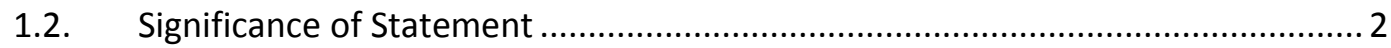

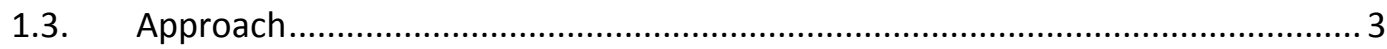

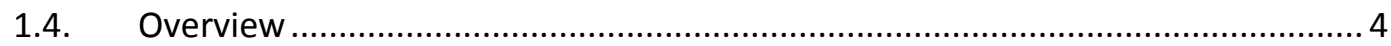

Chapter 2:

Literature Survey

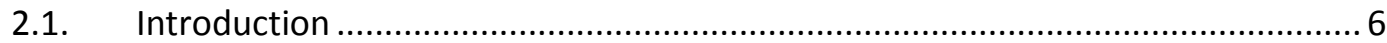

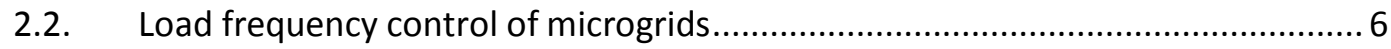

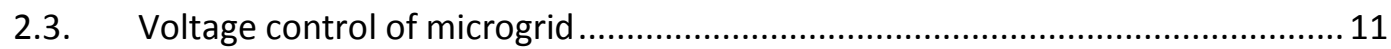

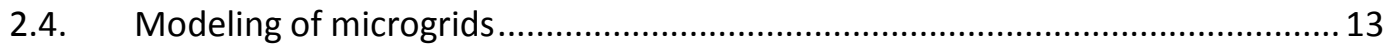

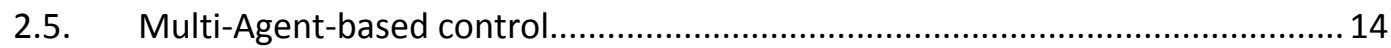

Chapter 3:

Comprehensive Modeling of Microgrids with VSI and PQ Inverters

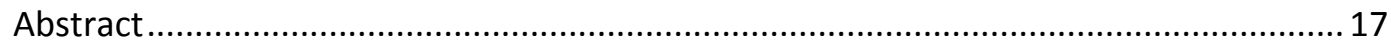

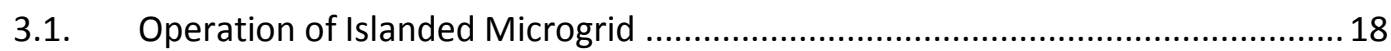

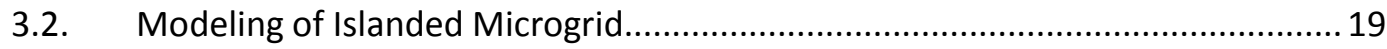

3.2.1. Modeling of Voltage Source Inverters (VSI) ......................................... 20

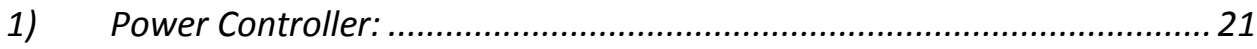


2) Voltage and Current Controllers: ....................................................... 23

3) Output LC Filter and Coupling Inductance: ...........................................26

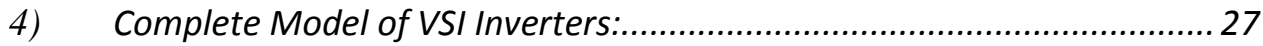

3.2.2. Modeling of $\mathrm{PQ}$ or Current Source Inverter (CSI) ................................. 28

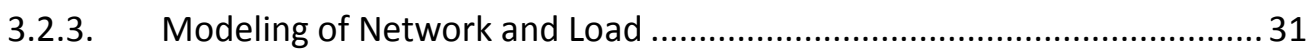

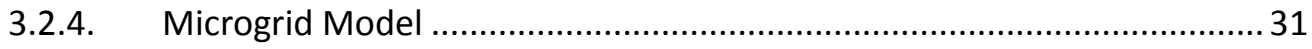

Chapter 4:

Load Frequency Control Analysis of Islanded Microgrid

4.1. Primary and Secondary Frequency Control Concepts ...................................... 34

4.2. Implementation and Analysis of secondary Frequency control ......................... 36

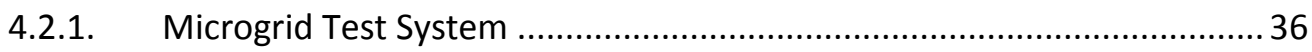

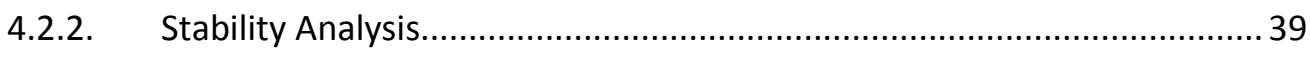

4.2.3. Small Signal Transient Analysis ....................................................... 42

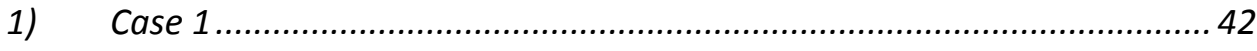

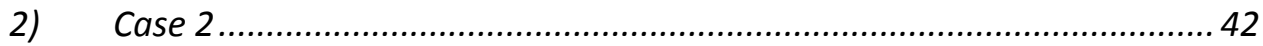

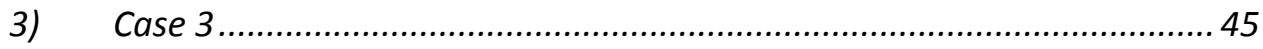

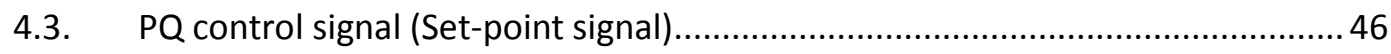

4.3.1. Simulation Result ....................................................................... 49

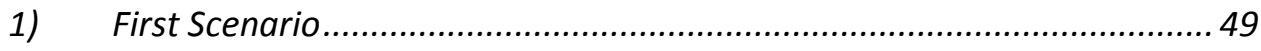

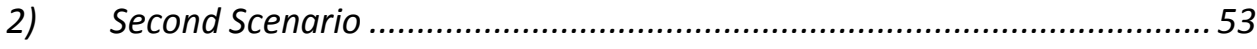

4.4. Novel Load Frequency control of microgrid with Tie Line Error ...........................54

4.4.1. Microgrid Test System …................................................................... 54

Modeling the Detailed Microgrid in PSCAD ${ }^{\circledR}$ Software ...................................... 55

4.4.2. Novel Load Frequency Control Design ................................................. 56

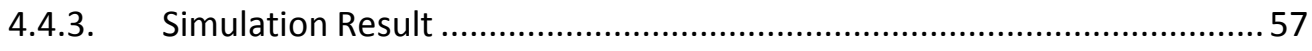


$>\quad$ First scenario

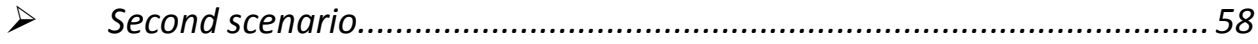

$>\quad$ Third scenario........................................................................... 58

4.5. State Space Formation of Microgrids Considering Virtual Tie Lines.................. 62

4.5.1. PI controller..................................................................... 62

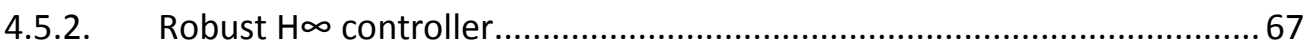

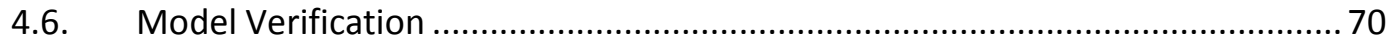

4.6.1. Detailed model (in PSCAD) VS Average model (in MATLAB) ................ 71

Chapter 5:

Control of Microgrid Voltage Profile using Consensus-Based Algorithm

Consensus Problem in Networks of Agents ...................................................... 74

Application of Consensus Solution for Secondary Voltage Control .............................. 74

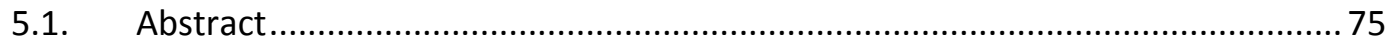

5.2. Optimum network structure for multi agents ....................................... 76

5.2.1. Consensus Problem on a Graph .................................................. 76

1) Undirected and directed graph properties .......................................... 79

5.2.2. Performance Measurement of Consensus Protocol ........................... 80

1) Mirror Graph Operation ............................................................ 81

2) Tradeoff between communication cost and connectivity........................... 81

5.2.3. appropriate network design Considering the Defined Criteria............... 81

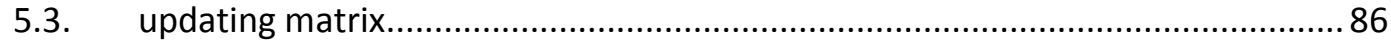

5.3.1. Coefficients setting of updating matrix....................................... 86

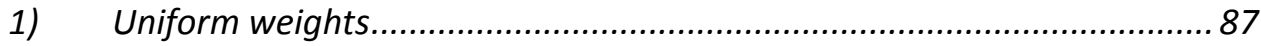

2) Maximum-Out Degree weight ...................................................... 87

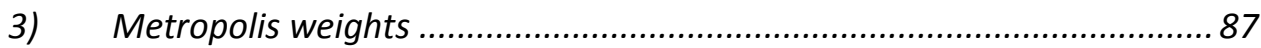




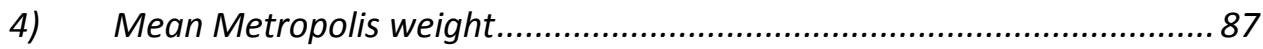

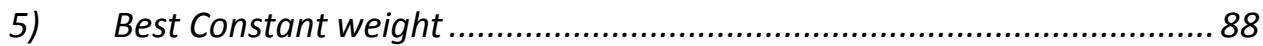

5.3.2. Iteration comparison of different updating rules ................................... 88

5.4. Time Delays of information sharing and reaching to an agreement .................. 91

5.4.1. Different delays of the system ........................................................ 91

5.4.2. Channel's time delay effect on convergence of ACA ............................. 91

5.4.3. Agreement time-delay and negotiation speed .................................... 92

1) Negotiation Speed (needed iteration for reaching an agreement).......... 92

2) Agreement Time Delay.................................................................. 93

5.4.4. Controller time-delay and possible threshold for updating controller.....94

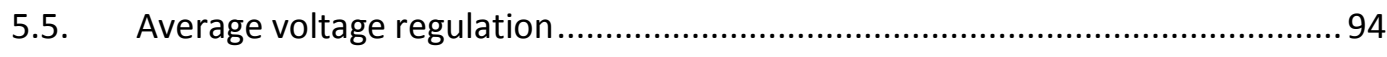

5.5.1. Average voltage regulation considering ideal agreement (No delay) ...... 95

5.5.2. Effect of consensus agreement time delay on average voltage regulation98

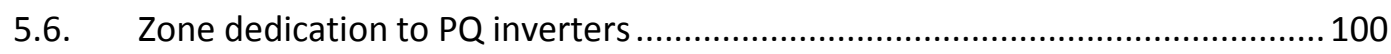

5.6.1. Required sensitivity analysis for zone definitions.............................. 103

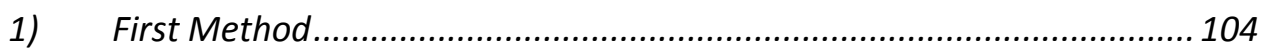

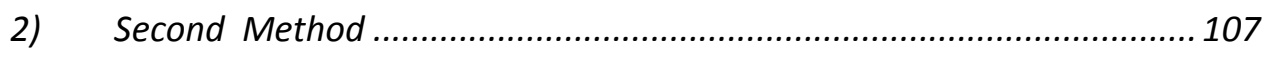

5.7. Simulation and results demonstration ................................................... 108

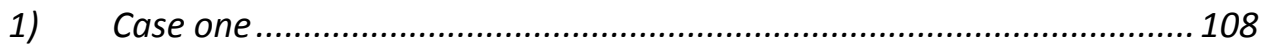

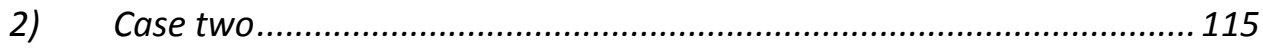

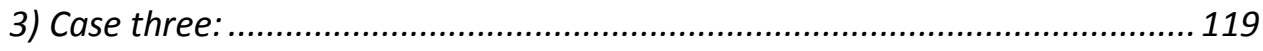

Chapter 6:

Appendices

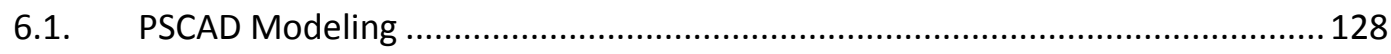

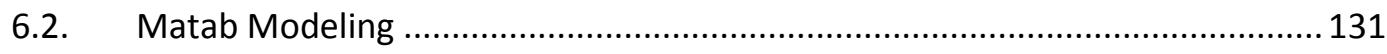




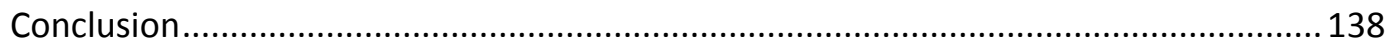

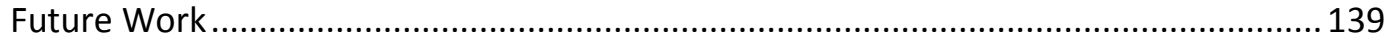

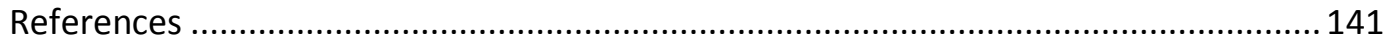




\section{LIST OF FigURES}

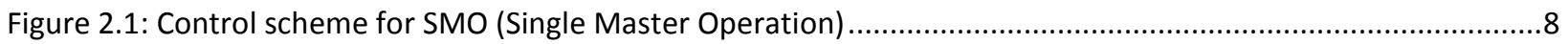

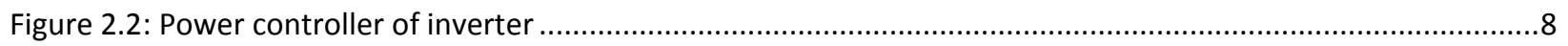

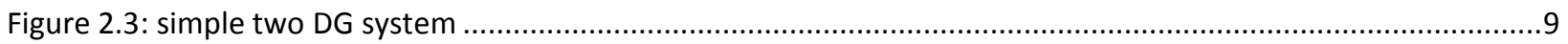

Figure 2.4: secondary control loop structure ..............................................................................................

Figure 2.5: Block diagram of the distributed secondary voltage control of VSI inverter in [2bidram] ......................12

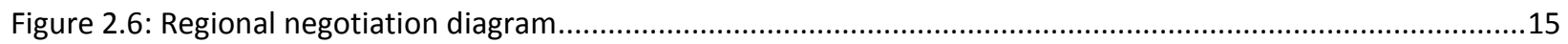

Figure 2.7: Frequency regulation of primary control in island mode .............................................................16

Figure 3.1: Control Scheme for Single Master Operation.................................................................................19

Figure 3.2: Block diagram of small signal state space model related to the microgrid component .........................19

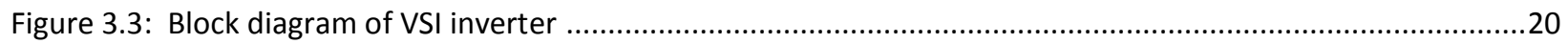

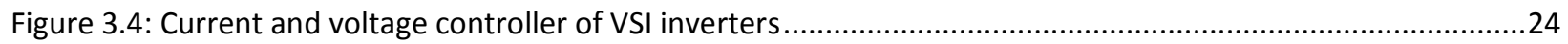

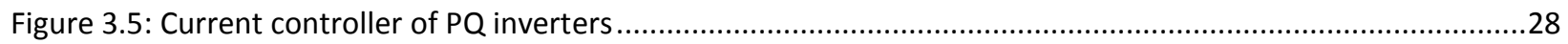

Figure 4.1: Secondary control for VSI inverters-generating units and energy storage devices .................................36

Figure 4.2: Steps of simulation and analysis procedure along with the implementation environments ...................37

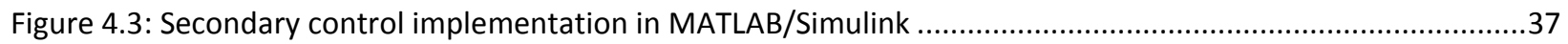

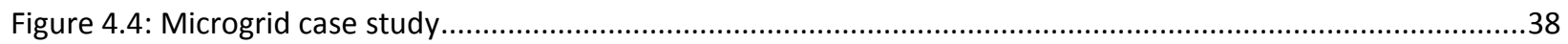

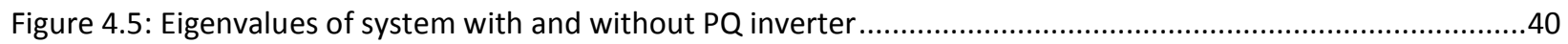

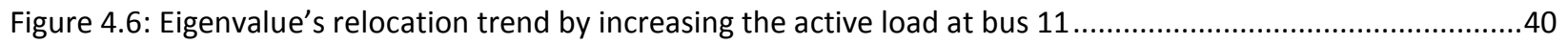

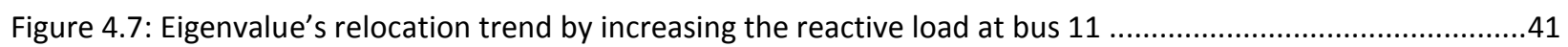

Figure 4.8: Eigenvalue's relocation trend by increasing the PQ inverter's participation factor ...............................41

Figure 4.9: Case 1-Secondary control for VSI 1, 2 and 3 (all of them are generating units) ...................................43

Figure 4.10: Case 2- Secondary control for VSI 1,2 and 3 (1 and 2 are generating units and 3 is energy storage device)

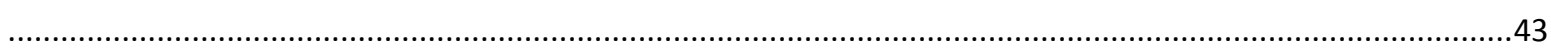

Figure 4.11: Output power changes of inverters in case 1 and 2. (Solid lines: case 1, Broken lines: Case 2) ............44

Figure 4.12: Change in nominal frequency set-points in case 1 and 2. (Solid lines: case 1, Broken lines: Case 2)......44

Figure 4.13: Frequency Error of VSI inverters in case 1 and 2. (Solid lines: case 1, Broken lines: Case 2) ................44

Figure 4.14: Change of delta in case 1 and 2. (Solid lines: case 1, Broken lines: Case 2) .....................................44

Figure 4.15: Frequency error of VSI inverters with and without $\mathrm{PQ}$ inverters participation................................45

Figure 4.16: Output power of VSI and PQ inverters with and without PQ inverter Participation ...........................45

Figure 4.17: Required communication for CSI control in the first solution ....................................................46

Figure 4.18: Required communication for CSI control in the second solution ................................................48

Figure 4.19: Required communication for CSI control in the third solution....................................................49

Figure 4.20: Reference frequency deviation of all VSI inverters of microgrid for four cases provided for signaling the $P Q$. ..49

Figure 4.21: Reference frequency deviation of VSI 1 during all four cases provided for signaling the PQ inverter ....50 Figure 4.22: Reference frequency deviation of VSI 2 during all four cases provided for signaling the PQ inverter....50 Figure 4.23: Reference frequency deviation of VSI 3 during all four cases provided for signaling the PQ inverter....51 Figure 4.24: Change of delta related to VSI 2 of the microgrid for all four cases provided for signaling the PQ inverter

Figure 4.25: Change of output active power related to VSI 1 of the microgrid for all four cases provided for signaling the $P Q$ inverter. . .52

Figure 4.26: Change of output active power related to VSI 2 of the microgrid for all four cases provided for signaling the $P Q$ inverter. 
Figure 4.27: Change of output active power related to VSI 3 of the microgrid for all four cases provided for signaling

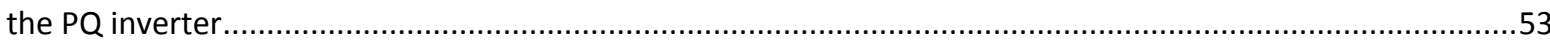

Figure 4.28: Bar graphs related to objective function values in different scenarios ..............................................54

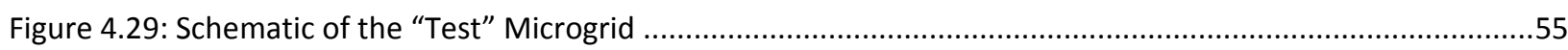

Figure 4.30: Schematic Block Diagram of a Three Phase Microgrid System .........................................................55

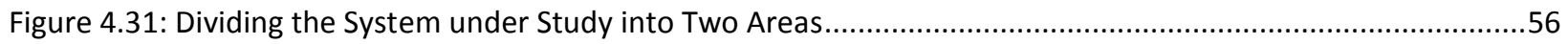

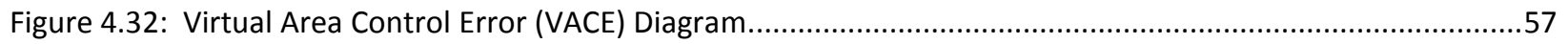

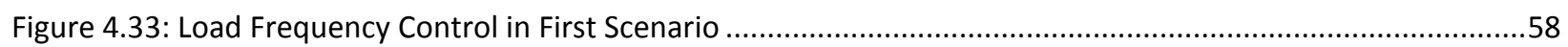

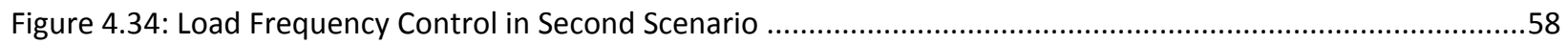

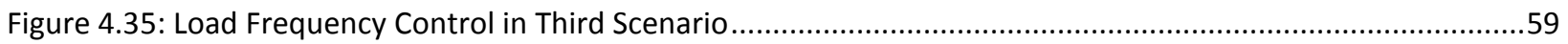

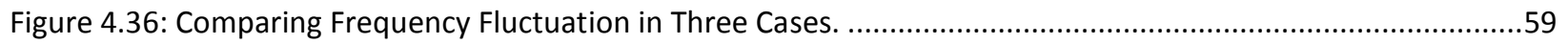

Figure 4.37: Comparing Torque of Synchronous Machine in Three Cases. .............................................................60

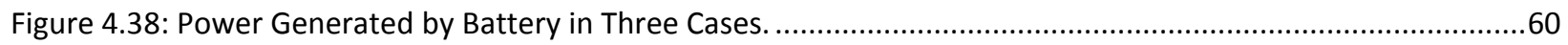

Figure 4.39: Power Generated by Synchronous Machine in Three Cases. ..........................................................60

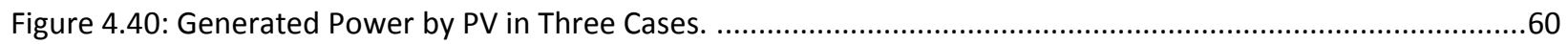

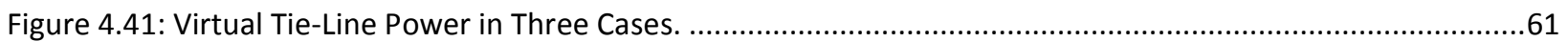

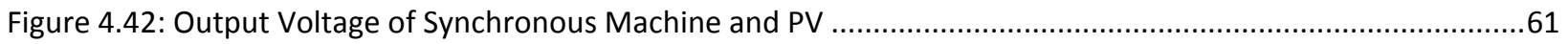

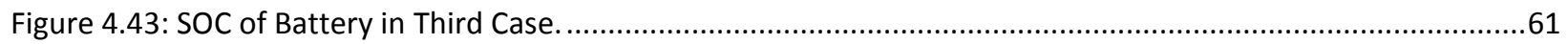

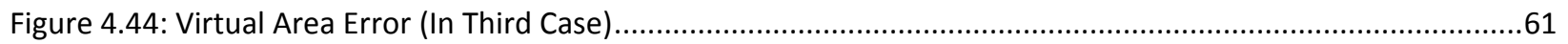

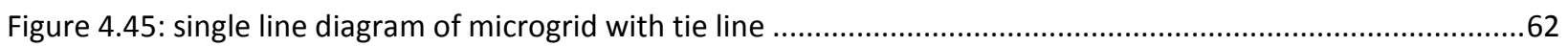

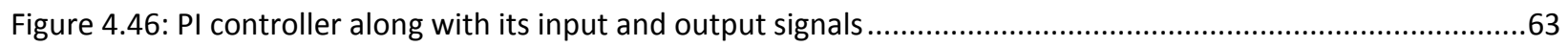

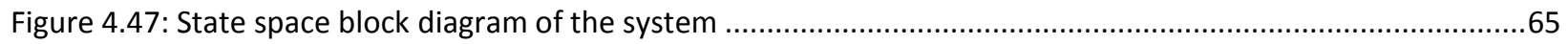

Figure 4.48: Output power of generating unit with and without tie line error (Solid lines: with tie line error - Broken

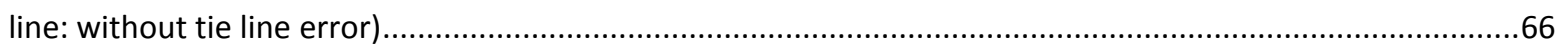

Figure 4.49: Frequency deviation with and without tie line error (Solid lines: with tie line error - Broken line: without

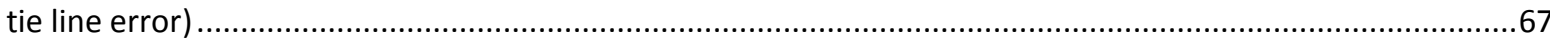

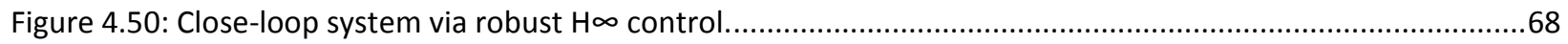

Figure 4.51: Frequency deviation with and without $\mathrm{H}_{\infty}$ (Solid lines: with PI controller-Broken line: with $\mathrm{H}_{\infty}$

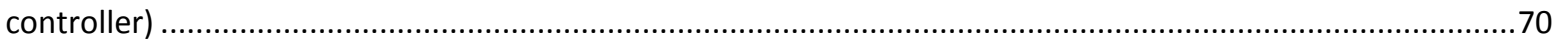

Figure 4.52: Frequency error for three scenarios (right and left figures are related to the average and detailed model)

. .71

Figure 4.53: Tie line power for three scenarios (right and left figures are related to the average and detailed model

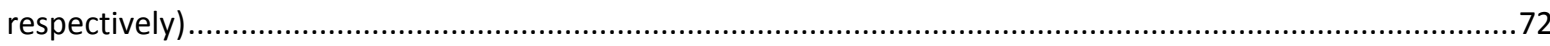

Figure 4.54: Power generated by battery for three scenarios (right and left figures are related to the average and

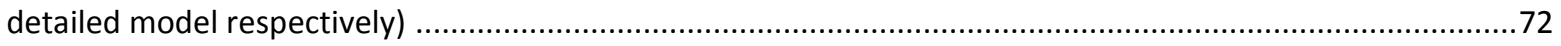

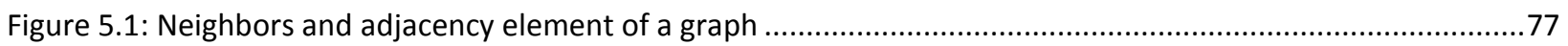

Figure 5.2: Flowchart related to the network design problem of consensus protocol ............................................83

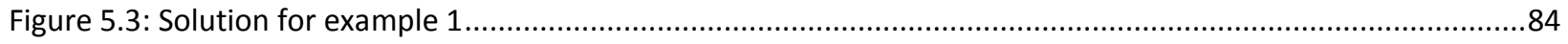

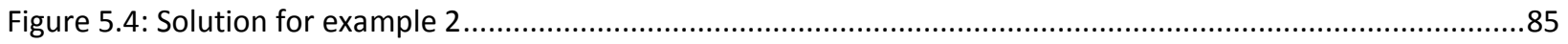

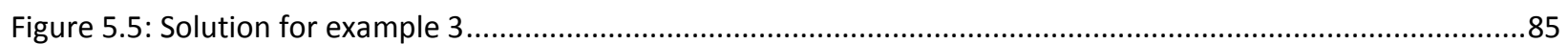

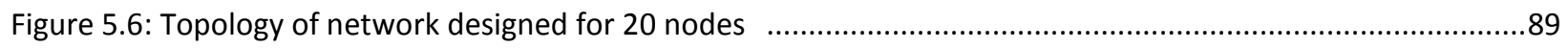

Figure 5.7: The convergence of different updating methods for network of 20 nodes .........................................89

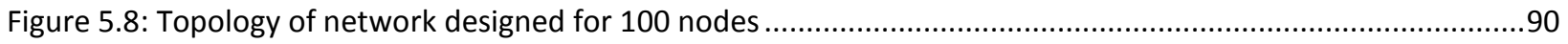

Figure 5.9: The convergence of different updating methods for network of 100 nodes ...........................................90

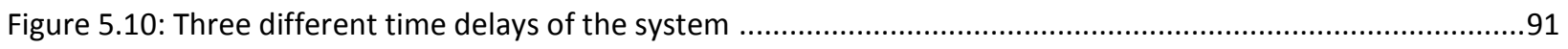

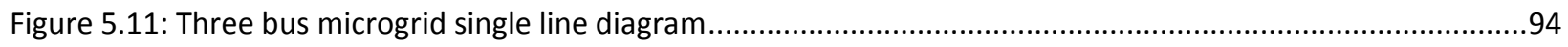


Figure 5.12: Primary and secondary control sections of one VSI inverter

Figure 5.13: Voltages at inverters terminal with (a) and without (b) using the consensus-based average voltage controller ..96

Figure 5.14: Voltages of buses with (a) and without (b) using the consensus-based average voltage controller .......96

Figure 5.15: Active power produced by VSI and PQ inverters with and without using the consensus-based average voltage controller (solid lines are representing the ACA based solution and dotted line is without ACA) ........97

Figure 5.16: Reactive power produced by VSI and PQ inverters with and without using the consensus-based average voltage controller (solid lines are representing the ACA based solution and dotted line is without ACA) ........98

Figure 5.17: Voltages of buses with different agreement time delays using the ACA-based average voltage controller .99

Figure 5.18: Voltages of buses plotted on the same graph using consensus-based average voltage controller ........99 Figure 5.19: Understudied 13 bus microgrid with two PQ inverters and two VSI inverters ..................................101

Figure 5.20: Zone dedication to $P Q$ and VSI inverters of the Understudied 13 bus microgrid considering the bus voltage sensitivity to the power production of inverters 102

Figure 5.21: Remained zones related to PQ inverters of the Understudied 13 bus microgrid along with their tie lines

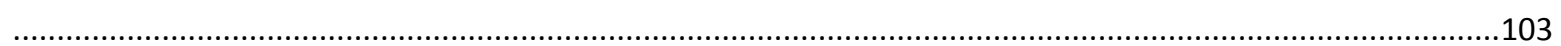

Figure 5.22: Load change location in case one and two ..........................................................................109

Figure 5.23: Voltages of main 6 buses with traditional voltage control (keeping VSI node voltages at 323.4 volt rms

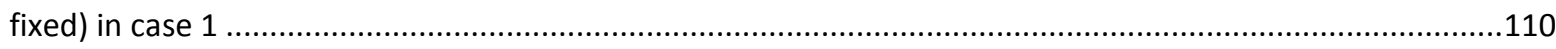

Figure 5.24: Voltages of main 6 buses with consensus-based voltage control (keeping the average voltages at 323.4

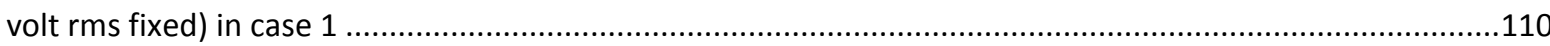

Figure 5.25: Voltages of main 6 buses with consensus-based voltage control along with zone dedication to PQ inverters in case 1

111

Figure 5.26: main bus voltages under different controller in case 1(dotted lines: traditional controller, solid line: consensus-based average voltage controller, broken line: zone dedicated method) .................................112

Figure 5.27: Active power produced by the four available inverters in the microgrid in case 1 ...........................113

Figure 5.28: Reactive power produced by the four available inverters in the microgrid in case 1 .......................114

Figure 5.29: Voltage profile of main buses with three different control scheme (traditional, consensus-based with

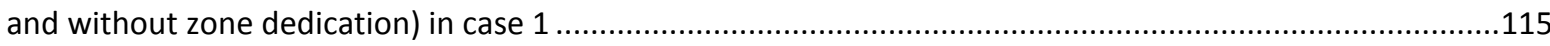

Figure 5.30: Voltages of main 6 buses with consensus-based voltage control (keeping the average voltages at 323.4 volt rms fixed) in case 2

116

Figure 5.31: Voltages of main 6 buses with consensus-based voltage control along with zone dedication to PQ inverters in case 2 116

Figure 32: main bus voltages under different controller in case 2 (solid line: consensus-based average voltage controller, broken line: zone dedicated method) ...............................................................................117

Figure 5.33: Active power produced by the four available inverters in the microgrid in case $2 \ldots \ldots \ldots \ldots \ldots \ldots \ldots \ldots . . . . . . . . . . . . . .118$

Figure 5.34: Reactive power produced by the four available inverters in the microgrid in case 2 .......................119

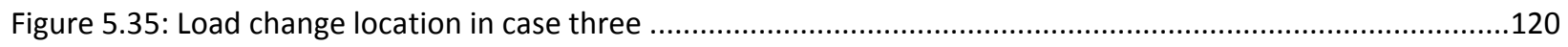

Figure 5.36: Voltages of main 6 buses with traditional voltage control (keeping VSI node voltages at 323.4 volt rms fixed) in case 3

Figure 5.37: Voltages of main 6 buses with consensus-based voltage control (keeping the average voltages at 323.4 volt rms fixed) in case 3

Figure 5.38: Voltages of main 6 buses with consensus-based voltage control along with zone dedication to PQ inverters in case 3

123

Figure 39: main bus voltages under different controller in case 3 (solid line: consensus-based average voltage controller, broken line: zone dedicated method) 124 
Figure 5.40: Active power produced by the four available inverters in the microgrid in case 3

Figure 5.41: Reactive power produced by the four available inverters in the microgrid in case 3

Figure 5.42: Voltage profile of main buses with three different control scheme (traditional, consensus-based with

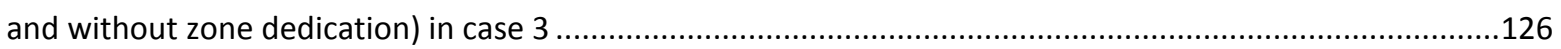

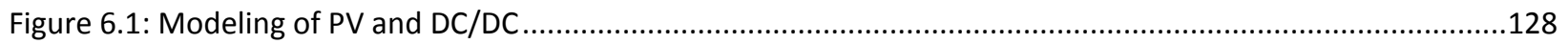

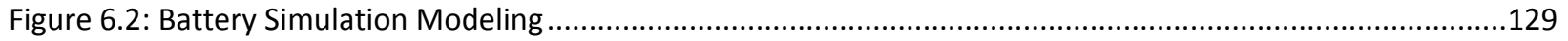

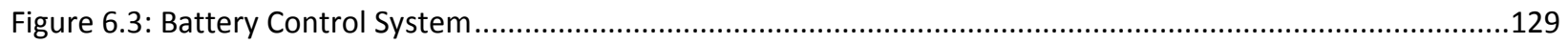

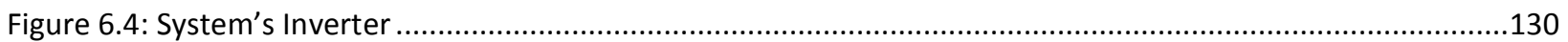

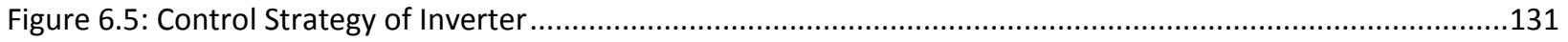




\section{LIST OF TABLES}

TABLE 4.1

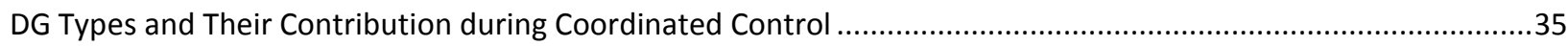

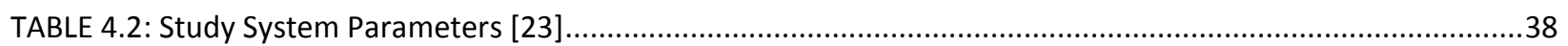

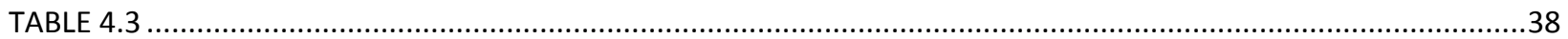

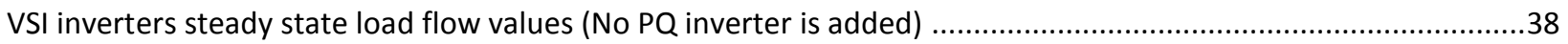

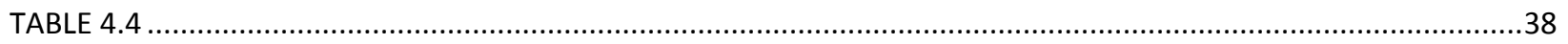

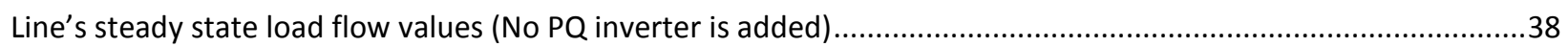

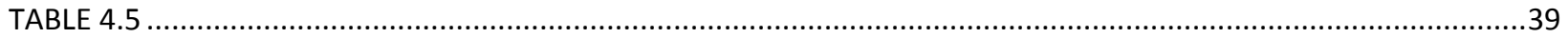

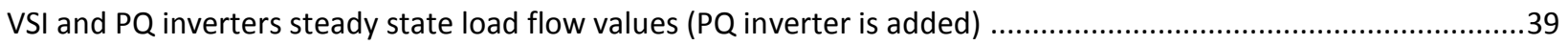

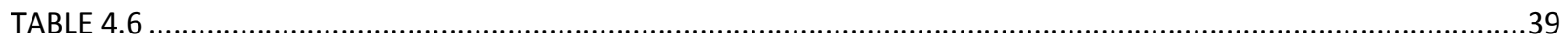

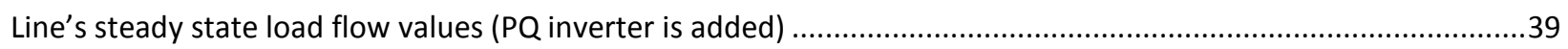

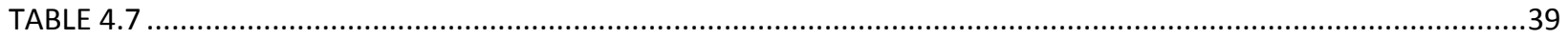

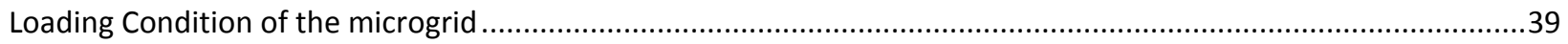

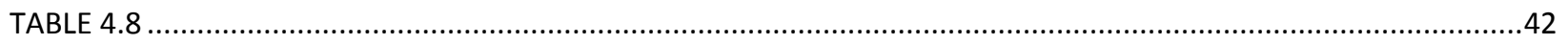

DG Types and Their Participation Factor during Coordinated Control in Second Case ..............................................42

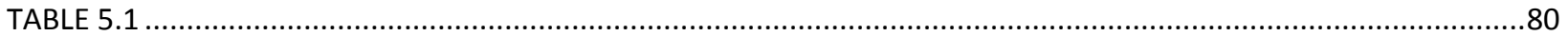

BALANCED AND UNBALANCED DIGRAPH EXAMPLE

TABLE 5.2 .

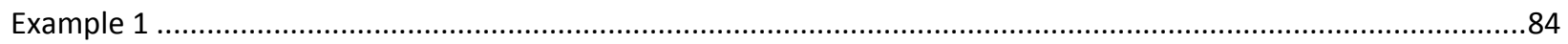

TABLE 5.3

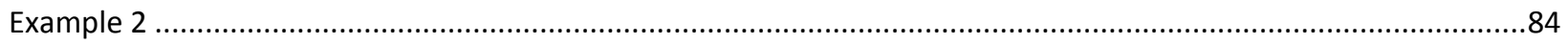

TABLE 5.4

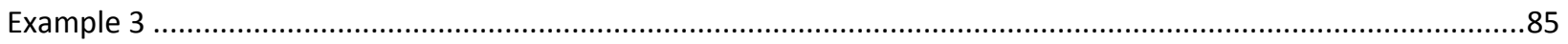

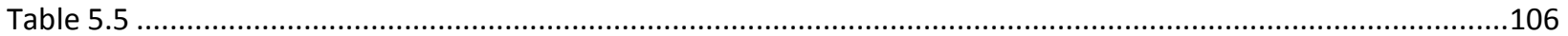

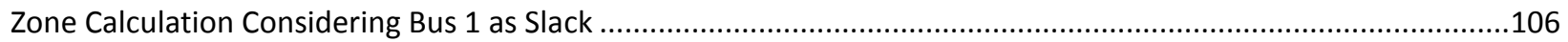

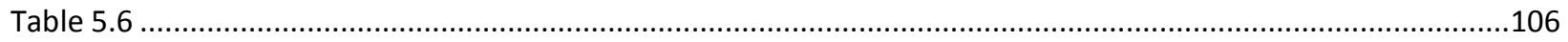

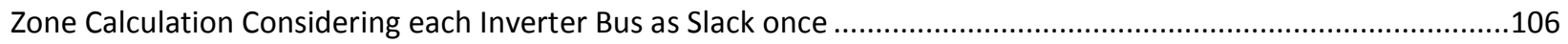

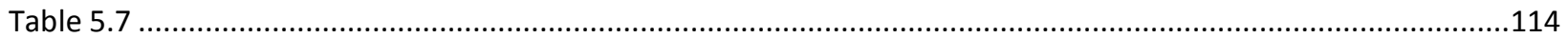

Steady state voltage value with and without zone dedication to PQ inverters for load increase in zone 4 ............114

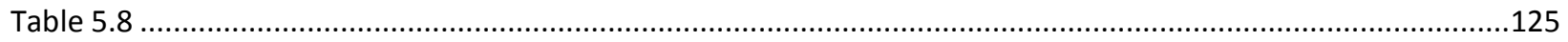

Steady state voltage value with and without zone dedication to $P Q$ inverters for load increase in zone 2 ............125 


\section{NOTATION AND ACRONYMS}

\begin{tabular}{|l|l|}
\hline LFC & Load Frequency Control \\
\hline MGCC & Micro Grid Central Controller \\
\hline VSI & Voltage Source Inverter \\
\hline DG & Distributed Generation \\
\hline DER & Distributed Energy Resources \\
\hline CSI & Current Source Inverter \\
\hline MA & Multi Agent \\
\hline RA & Reginal Agent \\
\hline LA & Local Agent \\
\hline SA & Service Agent \\
\hline CEI-DG & Constant Ellectronically Interfaced -DG \\
\hline PV & PhotoVoltaic \\
\hline BESS & Battery Energy Storage System \\
\hline PLL & Phase-Locked Loop \\
\hline PVECS & Photovoltaic Energy Conversion System \\
\hline SOC & State of Charge \\
\hline SPWM & Sinusodial Pulse Width Modulation \\
\hline STC & Standard Test Conditions \\
\hline MPPT & Maximum Power Point Tracking \\
\hline ACE & Area Control Error \\
\hline VACE & Virtual Area Control Error \\
\hline SMO & Single Master Operation \\
\hline MMO & Multi Master Operation \\
\hline ESS & Energy Storage System \\
\hline
\end{tabular}




\section{Chapter 1}

\section{Introduction}

\subsection{Problem Statement}

The integration of a variety of small-scale Distributed Generators (DGs) and storage devices, i.e. Distributed Energy Resources (DERs), in a power system results in drastic changes in the structure of traditional distribution networks. Different economic and environmental reasons are encouraging large penetration of DERs in microgrids [1-2]. These DERs allow the distribution system to operate in both gridconnected and islanded modes, adding more reliability and possibly supplying the load in off-grid mode [3]. However, this flexibility adds complexity to the control design and operation of the system.

During the operation of a power system, continuous changes in demand must be accompanied by adequate changes in generation to maintain the balance between generation and consumption. Since the load can change at any time, it is crucial to have an automated and robust balancing or a voltage and frequency control mechanism to adjust the power generation. Having such a mechanism is more significant when the microgrid is islanded [4]. Because of this need, designing appropriate control structures are becoming more 
pervasive and numerous, guaranteeing a proper set-point change of generating units to avoid instability of the microgrid. In this study, controllers are designed and installed on generating units and can affect the performance of the whole microgrid.

With the rapid growth of microgrids, the dynamic performance of control and methods of study have changed correspondingly. Designing and implementation of control techniques for DGs located far from each other to enhance the decentralized control is a more challenging problem than ever before. At the same time, one of the requirements of achieving the proper control is having appropriate modeling for the whole system. With regards to the above descriptions, the following questions are raised:

- Is there a systematic way to come up with a suitable model for the microgrid that subsequently can be used for controlling purposes?

- How is it possible to control a number of inverter-based generating units in a decentralized approach to maintain the demand-generation balance all the time?

One of the most practical and economical ways to achieve an appropriate load sharing between generating units in a distributed manner is using a droop characteristic for primary voltage and frequency control [56]. However, still it is not enough and it definitely needs some adjustment based on the micro source characteristics.

As described, the very first step being able to perform any kind of control techniques is having a general method to build any system's model. Most available modelings are specifically derived for one exclusive system. Although many of them are even detailed modeling, it is hard to expand them or change their controller structure, as they do not have state space matrices available.

\subsection{Significance of Statement}

The electric power grid has been selected by the engineering academy as the first choice for twentieth century's engineering innovation that is most beneficial to civilization [7-8]. The system with this level of attention and importance needs to be properly controlled to guarantee its normal functionality during change of operation point, contingencies and disturbances. Microgrids and smart grids are one of the most developing part of the electric power grid, which also have more complexities compared to the traditional generation. Microgrids contains so many power electronic devices and controlling them are far more complicated than the common mechanical system. In a microgrid, especially when it is disconnected from the main grid, any imbalance between the generation and demand can lead into voltage and frequency instability and several more load shedding if it is not properly controlled.

The dynamics of microgrids following considerable structural changes (plugging any DG's in and out) and loading conditions need to be controlled in order to ensure the system's integrity. These events can create stability problems in a fraction of a second. The inverters and other power electronic devices control also 
can act fast enough to maintain stability of a system due to their small time constants. Other than control of generating units of a microgrid, sometimes it is essential to manage the loads to prevent microgrid collapse or unnecessary load shedding at the time of power imbalance.

Categorizing DG's based on their characteristics and implementation of decentralized primary and secondary control of inverters in a microgrid improves the performance and provides more secure power supply for the loads.

\subsection{APPROACH}

The approach of this research is as follows. First, a comprehensive state space modeling is introduced by using and rearranging the equations related to the microgrid's components. There are a lot of modelings for microgrids and even though many of them are detailed, they have a main weakness, which is not having proper state space matrices defined. Designing controller for such a system is not easy because having state space matrices is the first requirement of designing an appropriate controller. So, during the modeling chapter of this study, a systematic way of forming state space matrices is presented. In this section, at first all the inverter-based micro sources are categorized as voltage controlled inverters (VSI) or current controlled inverters (CSI, also called PQ inverter) and their modelings are discussed. After finding out the matrices related to the inverters, it is necessary to join them all with other microgrid elements so it can form the whole microgrid state space matrices. After doing so, a decentralized secondary frequency control of system is achieved for the VSI inverters, meaning that all the information for control at each subsystem are being obtained locally and there is no need for remote signals from other subsystems. However, adding CSI inverters to the system, which play the slave role in a slave-master scenario, means some kind of communication is inevitable to signal the set-point for those inverters. The study conducted here tries to minimize the need for communication links as much as possible.

Meanwhile, a detailed model of a microgrid that was implemented in PSCAD software earlier was used for both modeling verification purposes and analyzing a novel concept for secondary frequency control. The aforementioned novel concept compares two different visions for the microgrid's control. The first vision is the common solution of considering the whole microgrid as one united electric power system in which all the micro sources have the same contribution based on their participation factors. The other vision, a more novel one, is trying to improve the transient response of system by dividing the microgrid to multi area systems and using the tie-line power error between them as a new term in Area Control Error (ACE). More than improving the transient response, there are also other reasons, which make this research necessary. By deregulation in distribution power market, the priority of micro sources for feeding loads will vary. So, as a result of this developing deregulation in distribution power market, the investigation of 
this novel load frequency control seems important. Moreover, this formation can help to reduce the frequency error by assigning an area to each fast response micro source in the microgrid.

Then by using the systematic solution described in the modeling chapter for forming microgrid's state space matrices, the concept of having multi area microgrid is implemented for the same detailed system. The state space matrices are defined in a way that the tie-line error is considered all through the modeling, which means it can be used as an input term for controller design. The results comparison with the detailed model of PSCAD can verify the accuracy of the proposed modeling. Finally, two different controllers are implemented, namely PI and H-infinity control. H-infinity control is used to counteract the effect of disturbances.

After discussing the primary and secondary frequency control of microgrids during the first and second chapters, it is important to consider the secondary voltage control of microgrids as well. So, this research dedicates the last chapter to distributed secondary voltage control of microgrids by proposing a consensusbased approach to guarantee proper voltage control and reactive power sharing among DGs. During this chapter, the droop control is used as the primary control to share the reactive power among VSIs based on some criteria such as rating; however, the secondary control is also needed to complete this task and compensate the remained voltage error. The consensus-based secondary control is implemented on the VSI inverters and relies on the data that they receive from their immediate neighboring. In spite of most other related communication-based control concepts, the proposed approach will only require sparse communication among DGs, which means central controller or all-to-all communication among DGs is no longer required. Each VSI inverter has an agent that is communicating and exchanging their data based on the consensus approach. The chosen protocol helps them to reach a global awareness about voltage and power sharing situation and subsequently, it enables the controller to make decisions based on the information it gets about the voltage.

Finally, after designing appropriate consensus-based secondary voltage control for VSI inverters, a zone dedication has been implemented to involve the PQ inverters in supplying the demanded load as well as VSI inverters and improve the voltage profile by taking the responsibility of dedicated areas to them.

\subsection{OVERVIEW}

The dissertation consists of the following chapters:

\section{$\checkmark$ Chapter 1: Introduction}

The introduction continues with an overview of outlines for this dissertation.

\section{$\checkmark$ Chapter 2: Literature Survey}

This chapter presents a survey concerning microgrid modeling and control. 
$\checkmark$ Chapter 3: Comprehensive Modeling of Microgrids with VSI and PQ inverters

$\checkmark$ Chapter 4: Load Frequency Control Analysis of Islanded Microgrid

This chapter will discuss the primary and secondary frequency control approach for VSI and PQ inverters inside a microgrid.

$\checkmark$ Chapter 5: Consensus-Based Average Voltage Control of Islanded Microgrid

The aim of this chapter is to address the distributed secondary voltage control of microgrid by use of consensus algorithm.

$\checkmark$ Chapter 6: Appendices

Appendices include system data, MATLAB computer codes and Simulink and PSCAD block diagrams. 


\section{Chapter 2}

\section{Literature Survey}

\subsection{INTRODUCTION}

In this chapter, a literature survey related to the problem investigated in this research is presented. The survey is organized as follows:

In section 2.2 and 2.3, works related to load frequency control and voltage control of microgrids are presented respectively. Then, in section 2.4, surveys on microgrid modeling is covered. Finally, in 2.5, the literature survey related to the multi-agent application in microgrid is discussed.

\subsection{LOAD FREQUENCY CONTROL OF MICROGRIDS}

In this part, a survey of studies related to development of Load Frequency Control (LFC) and power management techniques in micorgrid systems will be given. Generally, when operating a power system, continuous changes in demand must be accompanied by adequate changes in generation to maintain the balance between generation and consumption. Since the load can change at any time or any event such as a fault, or a sudden loss of a generating unit could happen unexpectedly, it is crucial to have an automated and robust balancing mechanism to adjust the active and reactive power generation. The need of such mechanism is more significant when the system is isolated-for example, an island in the middle of an ocean or a small rural area, which has no means of connecting to primary power grid. 
Several preceding studies presented different techniques of providing power management and/or LFC for small microgrids. A microgrid may comprise a variety of inverter interfaced distributed energy resources, energy storage devices and controllable loads. Controlling the inverters in a microgrid is a key point. For a microgrid with a single inverter, most of the traditional approaches use a voltage-frequency ( $\mathrm{v}-\mathrm{f})$ scheme in isolated operation or active power-reactive power (P-Q) control scheme in grid connected modes. It means that in islanded mode, the inverter should change its policy so that it can maintain the voltage and frequency at the nominal value.

Two kinds of control strategies can be used for inverters of a microgrid in isolated mode operation [9]:

- $P Q$ inverter control: the inverter is supposed to produce a pre-specified value for active and reactive power. These set-points for P and Q can be defined locally (using a local control loop) or centrally from the Micro Grid Central Controller (MGCC). Also, a group of micro sources connected to the main grid can operate all in PQ mode because the reference voltage and frequency is available.

- Voltage source inverter (VSI) control: the VSI emulates the behavior of a synchronous machine. It is controlled to feed the load with pre-defined values for voltage and frequency. As a result, despite having PQ inverters, the real and reactive output power is defined based on the load.

The equations below describe the frequency and voltage droop in a VSI inverter. Here $\omega_{0}$ and $v_{0}$ are nominal frequency and voltage respectively while $\omega$ and $v$ are representing the reference frequency and voltage and $\mathrm{k}$ is the droop gain.

$$
\begin{aligned}
& \omega=\omega_{0}-\mathrm{kP} \\
& \mathrm{v}=\mathrm{v}_{0}-\mathrm{kQ}
\end{aligned}
$$

If a cluster of VSI operates in a standalone AC system, frequency variations automatically lead to power sharing. An important point here is that in case of short circuit, the PQ inverter provides only a small amount of short circuit currents.

Study [9] also shows two possible control approaches for microgrids: Single master operation and Multimaster operation. In these approaches, the VSI inverters act as masters, and the other inverters operating in PQ mode are slaves. It is obvious that whenever there is a disturbance and the load changes in the microgrid, the PQ inverters produce their constant set-point as before in transient situation and the VSI inverters change their output power based on the frequency error, so all the load demand power is compensated with the VSI inverters (usually, the inverter of storage devices acts as a VSI inverter due to its fast response). This situation is not a desired condition for a steady state, because storage devices should only be responsible for the primary load frequency control as they have a finite storage capacity. Therefore, correcting permanent frequency deviation during islanded operation needs a secondary load frequency control. Secondary control loop changes the PQ inverter's set-point based on frequency deviations and allocates a new value for its set-point considering its rating and capability of producing power. Also, the same process 
happens in voltage control. Figure 2.1 can properly show the application of PQ and VSI inverter in a microgrid.

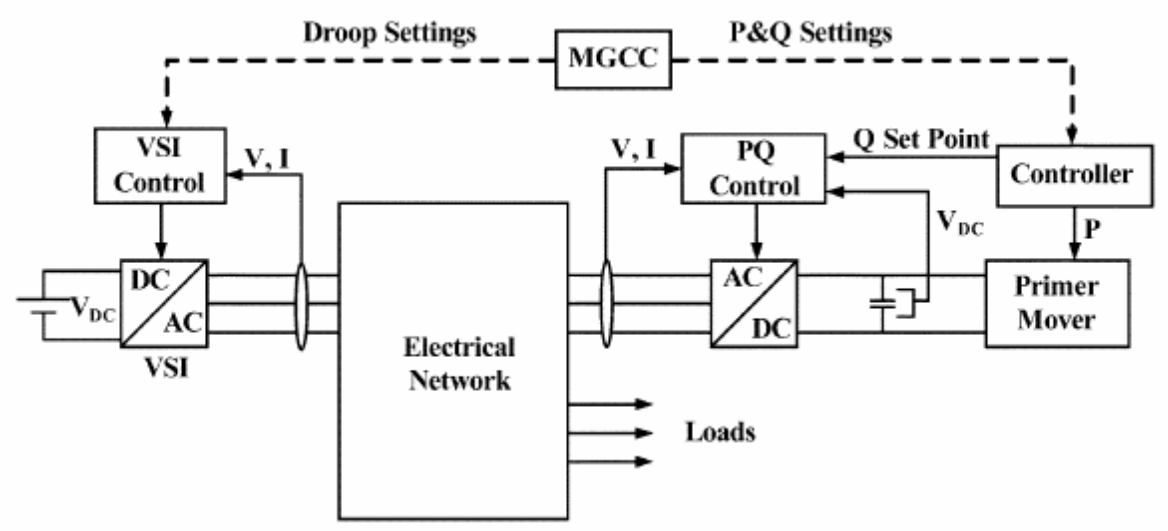

Figure 2.1: Control scheme for SMO (Single Master Operation)

Another solution for adjusting frequency violation is using the frequency error as a common signal among DGs to balance active power generation based on their droop coefficient. It should be noticed that the droop coefficients are usually determined by assuming the load demand is shared among DGs proportional to the capacity of units. This approach is not as efficient as the previous one because in this strategy, all DGs take part in the primary frequency regulation process. Due to the slow response of some of them, the transient response will not be as good as the other strategy in which only the fast response devices take action in primary frequency control loops. Figure 2.2 shows the diagram related to the control loops of the mentioned approach used in paper [10]. In this paper, the purpose is to restore the frequency of a microgrid to the nominal value while ensuring proper power sharing happens between multiple DGs considering the rating and dynamic characteristics of various energy sources. One of the critical control tasks of a microgrid is voltage and frequency regulation of the system. This paper uses the power vs. frequency droop control since it is easy to implement and it enables decentralized control of multiple distributed generations. The author believes that the power droop control alone is not enough for the microgrid because of continuous variation of load demand, so he suggests two different secondary load frequency control; the first one is using a central controller that sets the power reference signal of each DG and the other one is adding an integral controller to the existing droop controller. The second way is used by the author because of locally measured signals without requiring any further communication link.

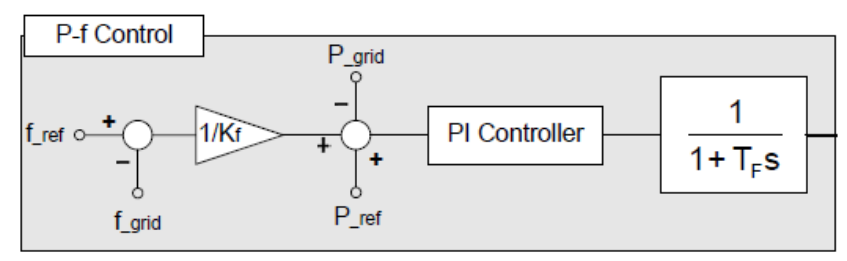

Figure 2.2: Power controller of inverter 


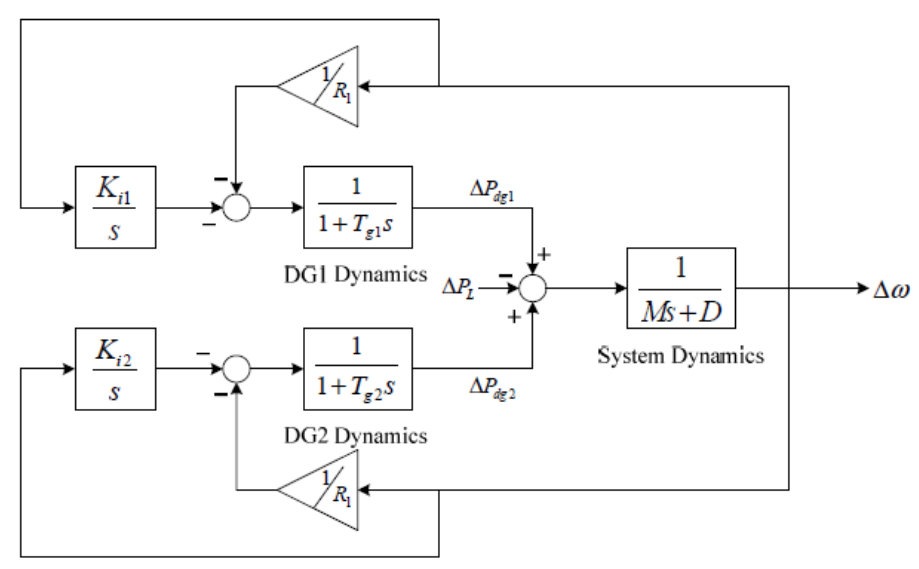

Figure 2.3: simple two DG system

Based on previously mentioned control strategies, whenever there was a single inverter in a microgrid, the inverter should use the VSI control in order to provide a reference value for voltage and frequency. However, paper [11] tries to implement a new power and voltage control (P-V) scheme for a single inverter that operates in the islanding mode. The proposed control scheme used by this paper is applied in the droop control method to handle the active power sharing among parallel inverter-interfaced distributed energy resources operating in islanded mode.

In [12], a power storage system is used with a solar photovoltaic, diesel generator and wind power generation for load frequency control of a power system. The microgrid system at Aichi Institute Technology in Japan is used as a simulation model. In this paper, secondary load frequency control was performed using two different methods. The first one is a power demand estimation and the second one is proportion control, which uses the frequency deviation. In proportional control, the purpose is bringing the frequency back to zero, which may not be the best solution when the load is fluctuating. The author has used proportional control in the absence of storage devices. Then, the results are compared to the situation, where there is storage in the system and the load demand estimation method is applied for load frequency control. In load demand estimation, the load fluctuation of an area is estimated (the estimation of some generation units like PV and wind is also needed) and it has been used for the output set-point of the storage device, so that it can compensate for this change. The drawback of the load demand estimation method is that load changes are not usually available in microgrids.

Sedghisigarchi and Feliachi[13] introduced a distribution area error (DSE) to formulate the frequency control problem. Two control loops are proposed, the first one guaranteeing that the fuel cell is protected by maintaining its cell utilization within its admissible range and the second one tracking the load and regulating the frequency. Then, the DSE error is used by the secondary control loop. In this paper, the author tries to present a distributed load frequency problem with its own distribution system error. Fuel cells are used as DG here, which is an inverter-based generation unit and its output power can be controlled 
by adjusting the firing angle of the inverter. The secondary loop controller is designed for the fuel cell to correct the frequency deviations by tuning the inverter's firing angle. In this paper, a number of distributed generators are supplying a portion of the native load while the remaining power is provided by the substation. Distribution Area Error, which is a measure to assess performance, is described as below:

$$
\operatorname{DSE}=|\Delta \mathrm{f}|+\beta|\Delta \mathrm{Ps}|
$$

Where Ps is power coming from the substation and $\Delta \mathrm{f}$ represent frequency error.

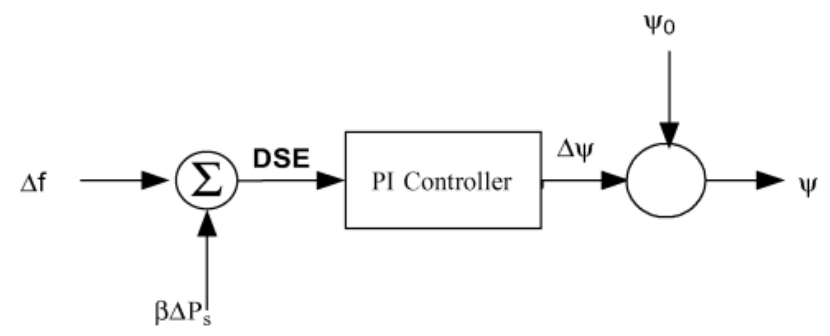

Figure 2.4: secondary control loop structure

As discussed, the microgrid is a potential solution for future distributed generation systems. It aggregates many distributed energy resources (DER), like renewable sources, and loads as an autonomous region. However, controlling a microgrid is still a complex issue and many proposed solutions, are only based on locally measured signals without any communications; thus, it is difficult to achieve global optimization. Multi-agent systems are introduced in several papers in order to be implemented in microgrids because a system with multiple interacting intelligent agents can solve problems that are difficult or impossible for an individual agent. In [14-17], Dimeas et al. proposed a three-level Multi-Agent System (MAS) for microgrid control. Their research focuses on microgrid market operations using an auction algorithm to share the power among the DER units, presenting how the local intelligence and the social ability of the agents may provide solutions in the optimal and effective control. The strategy is only adapted in grid-connected mode, because the extra energy is bought or sold to the grid that is assumed to offer or receive infinite energy. The aforementioned papers proposed a complete decentralized solution; however, many recent proposed solutions use a centralized architecture that requires expensive and reliable communication systems. For example, in [18], a three-level hierarchical control is proposed and in [19], Tsikalakis et al. suggested an optimal active and reactive power control strategy that is based on a centralized solution. In order to avoid the disadvantages of centralized solutions, a distributed multi-agent system approach is discussed in [1921]. Nowadays distributed control of microgrids with increased penetration of DERs is becoming more and more popular. Distributed control uses local communication networks and includes the positive features of both centralized and decentralized controls [22]. In [19], the authors used a multi-agent system for frequency control of islanded microgrid where each agent needs to know the global information of the 
microgrid while communicating only with neighbors. Then an optimized average consensus algorithm is used, which relies on local information and guarantees to estimate the required global information.

\subsection{VOLTAGE CONTROL OF MICROGRID}

Proper control of microgrids is a prerequisite for stable and economically efficient operation of smart grids. Once a microgrid is islanded, the so-called primary control maintains the stability of voltage and frequency. However, even in the presence of a primary control, voltage and frequency can still deviate from their nominal values. To restore the voltage and frequency of DGs to their nominal values, the so-called secondary control is also required.

Usually, in most articles, voltage and frequency droop controls are used as the primary control for both frequency and voltage to maintain the stability of the system [20,23, and 24]. Even though the primary control is mostly the same and completely decentralized to make sure the stability of the system is not dependent on the communication network, the secondary control is different and it suggests a variety of solutions from having central controller, distributed one with sparse communication network or a completely decentralized.

Bidram et.al. in [20] introduced a distributed cooperative control of multi-agent systems, which implements the secondary voltage control of microgrids. By considering DGs as agents in a microgrid, he represented the secondary control of microgrids as a tracking synchronization problem where all DGs try to synchronize their terminal voltage amplitude to a pre-specified reference value. For this purpose, each DG needs to communicate only with its neighbors. The primary voltage control is based on droop characteristic, which has a reference voltage; however, in order to apply the secondary control, the author had to choose an appropriate value for the control input (reference voltage) to implement the secondary voltage control. The purpose of the secondary control in this paper is only regulating the terminal voltage to the reference value. He improved his work later around, in [25], by bringing another term to the error so he could implement the reactive power sharing as well as voltage control. Figure 2.5 shows the implemented secondary voltage control in his latest work [25]. The error formula used in [20] and [25] are also shown in equations 2.4 and 2.5 respectively. $v_{o d i}$ and $v_{o q i}$ are actual voltages of d-q axis and $v *_{o d i}$ and $v *_{o q i}$ represent the reference voltages of d-q axis. Finally, nqi is the droop of voltage at node $i$. 


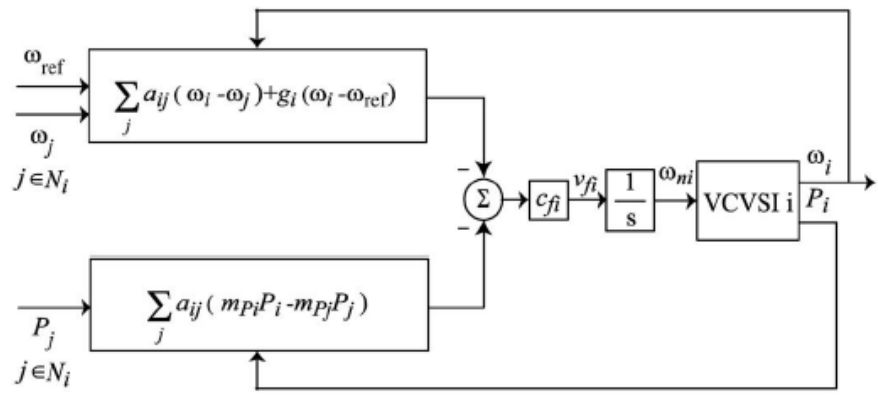

Figure 2.5: Block diagram of the distributed secondary voltage control of VSI inverter in [2bidram]

$$
\begin{aligned}
& e_{i}=\sum_{j \in N_{i}} a_{i j}\left(\left[\begin{array}{c}
v_{\text {odi }} \\
v_{\text {odi }}^{*}
\end{array}\right]-\left[\begin{array}{c}
v_{\text {odj }} \\
v_{\text {odj }}^{*}
\end{array}\right]\right)+g_{i}\left(\left[\begin{array}{c}
v_{\text {odi }} \\
v_{\text {odi }}^{*}
\end{array}\right]-\left[\begin{array}{c}
v_{\text {ref }} \\
0
\end{array}\right]\right) \\
& e_{i}=\sum_{j \in N_{i}} a_{i j}\left(v_{\text {odi }}-v_{\text {odj }}\right)+g_{i}\left(v_{\text {odi }}-v_{r e f}\right)+\sum_{j \in N_{i}} a_{i j}\left(n_{Q_{i}} Q_{i}-n_{Q_{j}} Q_{j}\right)
\end{aligned}
$$

Another difference happens when taking PQ inverter into account. A two-layer, multi-objective control framework is introduced for VSI and PQ inverters. The decoupled control framework individually regulates the voltage/frequency of VSI inverters and active/reactive powers of PQ inverters. These controllers are implemented through a communication network with a one-way communication link. In first network layer related to VSI control, a reference value for voltage is required while in the second layer of the network, the reference value determines the participation of PQ inverters.

As mentioned previously, most researchers used voltage droop controls as the primary control. However, there are some works such as [26] that replaced the primary droop control with a so-called Distributed Voltage Control (DVC), which is a consensus-based solution for the problem of reactive power sharing in autonomous inverter-based microgrids. The proposed control required sparse communication even for the primary control. It uses weighted average consensus protocol for reactive power sharing and at the same time, by selecting criteria for the control parameters, it makes the average of all voltage amplitudes in the network equivalent to the nominal voltage amplitude. This solution that considered DVC as an alternative for both primary (voltage droop control) and secondary control makes the system less reliable because of the presence of communication links even though it is sparse. Voltage droop control is mainly used by researchers as a primary control because of the totally decentralized structure that provides a confident situation in case of secondary control failure for any reason, such as a loss of communication links. The equations $(2.6,2.7)$ show the error term used between agents for power sharing purpose by [26].

Where $Q^{m}{ }_{i}$ and $\chi_{i}$ are the generated reactive power and reactive power rating of node i respectively. 


$$
\begin{aligned}
& u_{i}^{V}=V_{i}^{d}-k_{i} \int_{0}^{t} e_{i}(\tau) d \tau \\
& e_{i}(t)=\sum_{k \sim C_{i}}\left(\frac{Q_{i}^{m}(t)}{\chi_{i}}-\frac{Q_{k}{ }^{m}(t)}{\chi_{k}}\right)
\end{aligned}
$$

It is good to mention that, in [24], Abdel-Rady et.al proposed a power-sharing strategy that is based on static droop characteristic combined with an adaptive transient droop function. So, even though the author is using the droop characteristic for its primary control, he is trying to implement an adaptive decentralized droop control instead of the conventional static one. Unlike the conventional droop controllers, which yield 1-DOF tunable controllers, it has the opportunity of a 2-DOF tunable controller. So, without affecting the static droop gain, it helps to damp the oscillatory modes of the power controller. The proposed equations for droop function discussed in [24] are shown in equations 2.8 and 2.9.

Where $\widehat{m_{d}}$ and $\widehat{n_{d}}$ are dynamic droops gains related to the voltage and frequency while $m$ and $n$ represent the static values.

$$
\begin{gathered}
\omega_{0}=\omega^{*}-m P-\hat{m}_{d} \frac{d P}{d t} \\
V_{o d}^{*}=V^{*}-n Q-\hat{n}_{d} \frac{d Q}{d t}
\end{gathered}
$$

All of the above papers in voltage control area assumed that the loading in the original network is such that no power or voltage constraints are violated at any time. So, they are not concerned about possible requirements of considering load shedding in the microgrid. Lie and $\mathrm{Gu}$ in [19] proposed a multi-agent, system-based frequency control strategy that is decentralized and needs only sparse communication links between neighbors. The important point considered in this article is that other than primary and secondary frequency control, load shedding is also executed by means of global information discovered through agents. However, it has been done only for frequency regulation purposes. It can be a good idea to implement such a consideration of load shedding in voltage control.

\subsection{MODELING OF MICROGRIDS}

In order to develop a control strategy for a microgrid, an appropriate state-space model is needed. There are few works focusing on inverter-based microgrid modeling [23, 27, and 28]. In [23], Pogaku et.al. presented a modeling and analysis of autonomous operation of inverter-based microgrids. The model captures details of the inverter's control loops but not the switching action. This paper has modeled only Voltage Source Inverters (VSI), and the Current Source Inverters (PQ) are not taken into account; 
furthermore, the effect of changing nominal frequency and voltage set-points in state space modeling are not considered, which prevents implementing the secondary control.

In [20], a distributed cooperative control algorithm has been introduced using a nonlinear model and the feedback linearization approach. This paper does not consider the PQ inverters during the modeling. In a follow-up paper [25] the authors added the PQ inverter model and analyzed its effects on the secondary control. However, this modeling is used only for analysis as opposed to the purpose of this dissertation, which is stability enhancement and control design. It should be also noted that the secondary control proposed in $[20,25]$ does not differentiate between storage devices and generating units, which have distinct characteristics as proposed in this research.

\subsection{MULTI-AgENT-BASED CONTROL}

Multi-Agent (MA) control is an emerging field in power systems and has been reported in many applications, and some promising results were obtained in several areas including operation, markets, diagnosis and protection. With increasing complexities of power systems, there is more need for intelligent and learning controllers that can adapt themselves to different operating conditions and learn the proper control action in case of unpredicted situations. Therefore, making the conventional controllers more intelligent has been investigated in many research studies.

In classical load frequency controllers, a fixed gain for the controllers is used. Even with optimized values for the controller's gains, the controllers still cannot perform satisfactory when the operating condition of the system deviate from the nominal values. Also, the optimal controllers are functions of all system's states, which, in practice, may not be available. Therefore, along with various areas in power systems, intelligent controllers have also been applied for load frequency control purposes in order to make it applicable to a real system and compensate for the drawbacks of conventional controllers. Having intelligent agents and using them in a multi-agent scheme can improve the system in many aspects.

In [29], a multi-agent system is introduced for DERs control in a microgrid in both grid-connected mode and islanded mode. The DER units and loads are classified and three types of agents are defined, namely the regional agent (RA), the local agent (LA), and the service agent (SA). After having different agents, two-layer control strategies can be used to achieve local autonomy and global optimization respectively. An agent is capable of making decisions and acting independently and takes certain approaches to finish the task given according to some principles. The important characteristics of an agent are described below:

- Agents have a certain level of autonomy; they can function properly and control their internal status. For example, the agent controlling the battery unit can automatically cease generating power when energy storage has decreased to a certain level. 
- They can interact with each other or human being to achieve certain goals. A DG agent can change its set-point according to local needs and can also change the security level of the microgrid in a global view.

- The agents have self-adaptability, which is possible by a learning mechanism online or offline. In other words, a regional agent will evaluate the behavior of each subordinate agent and then stimulate or punish them, so they will learn to take the actions that bring about long-term benefits.

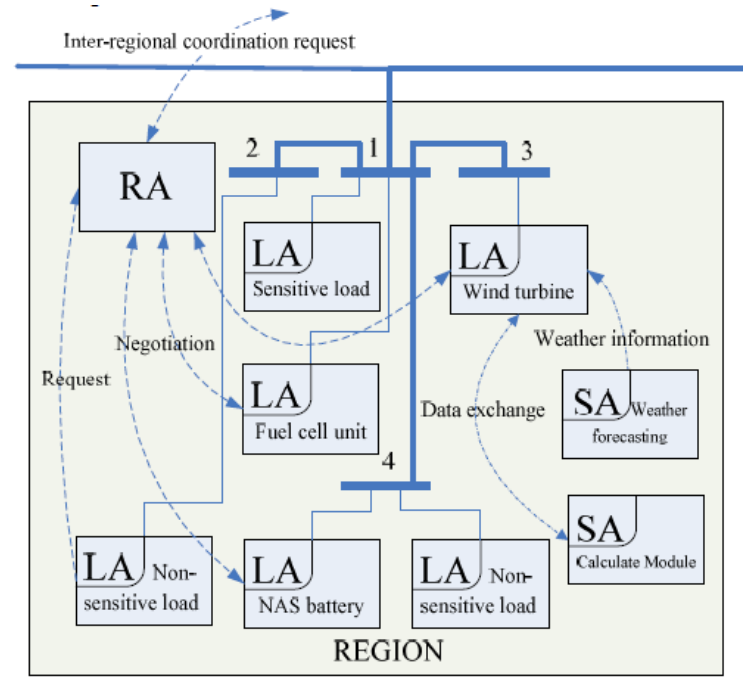

Figure 2.6: Regional negotiation diagram

In [29], during the primary control, the DG units-like PV and wind turbines, which are weather dependent - still operate in (PQ), generating as much power as possible on the premise of voltage stability. In contrast, the other Constant Electronically Interfaced DG units (CEI-DG), like fuel cells, storage units and conventional DG units in the microgrid, are responsible for frequency regulations. A real power vs. frequency droop characteristic is introduced here for CEI-DG units and available storage devices; this droop characteristic control cooperates with primary frequency regulations of conventional DG units to share real power proportional to their capacities. The initial power set-point of each frequency regulating DER is obtained from a database based on daily generation/load forecast in the microgrid. Due to frequency deviations caused by droop characteristic, a limited number of DERs with relatively larger capacities and faster responses are involved in frequency restoration, or also called secondary frequency regulation. 


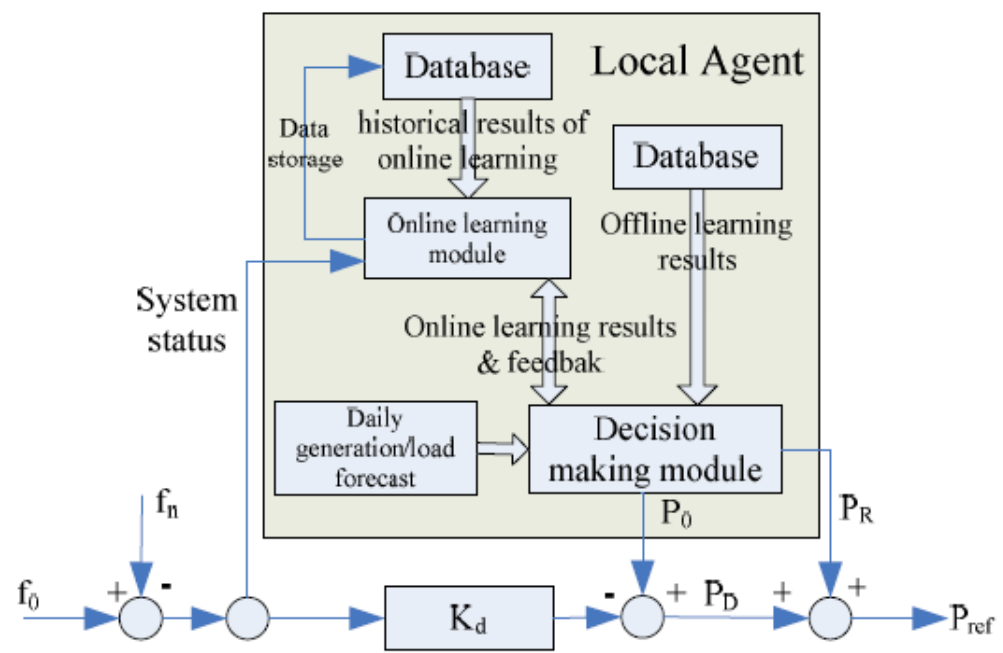

Figure 2.7: Frequency regulation of primary control in island mode

Here in this dissertation at first, a comprehensive state space model of a microgrid consisting of PQ and VSI inverters is developed and the relative stability is analyzed. In the sense of the small-signal dynamics of a microgrid, it can be shown that as the PQ inverter is added to the system, the low-frequency modes of the power-sharing dynamics drift to new locations and the relative stability is improved by giving a better stability margin to the system. The effect of changing the nominal value of voltage and frequency for VSI inverters and active and reactive power set-points for PQ inverters has been taken into account all through the modeling, which provides a proper input matrix to use for further control actions. Meanwhile, to preserve the power-sharing stability, a decentralized droop controller of paralleled VSI inverter units is presented. Then, the model is used to design and implement distributed secondary frequency controllers by means of the inputs provided during the modeling. Also, the consideration of storage device's nature on secondary control yields a stable long-term performance as they are not involved in power sharing in steady state situation. Then, for the secondary voltage control, an Average Consensus Algorithm (ACA) is used and applied to a network of agents that has been optimally chosen based on the required connectivity. The main purpose of the ACA is to keep the average voltage of all the buses at a desired level during islanding. Then, another control strategy is proposed to improve the voltage profile. While the average voltage is kept fixed by the voltage controlled inverters, this voltage profile smoothness is obtained by dedicating zones to current controlled inverters and defining their responsibilities based on the location of the loads.

The proposed modeling and control methodologies are demonstrated using an 11-bus microgrid with and without current controlled inverters. 


\title{
Chapter 3
}

\section{Comprehensive Modeling of Microgrids with VSI and PQ inverters}

\begin{abstract}
In this chapter, a comprehensive state space modeling of microgrids consisting of PQ and VSI inverters is developed first. The effect of changing the nominal set-points of voltage and frequency for VSI inverters and active and reactive power set-point for PQ inverters has been taken into account all through the modeling, which is then used to investigate the effect of different secondary controllers on the microgrid. This modeling also provides a convenient modeling tool for any further secondary load frequency and voltage analysis. Then, during the next chapter, the state space matrices of an 11-bus microgrid is formed to illustrate how the proposed modeling can be used for implementation of different control methodologies.
\end{abstract}




\subsection{OPERATION OF ISLANDED MICROGRID}

A typical characteristic of a microgrid is that it can be operated either in grid connected or in an islanded mode. Normally, in a grid connected mode the voltage and frequency are supplied by the main grid, so the inverters can act as constant power sources (PQ inverters). However, in an islanded mode, some of the inverters are controlled to supply the power needed by the local loads while maintaining the voltage and frequency within the nominal range (VSI inverters). Autonomous operation of a microgrid might be planned either due to maintenance or economics, or the operation might be unplanned due to faults in the main grid [23].

The voltage and frequency control of an islanded microgrid includes the primary and secondary controls as in a conventional power system. During the primary frequency control the fast response sources, such as storage devices, will help the microgrid maintain frequency stability quickly and automatically. However, the primary loop is not enough. The energy storage devices have to be recharged to increase the reserve power for dealing with emergencies. The frequency and voltage have to be regulated to the rated values. Also, the power sharing between DER's should be done based on some predefined criteria such as rating or economics. Obviously, another control level is necessary for restoring the voltage and frequency. A secondary control loop is needed to bring the voltage and frequency back to nominal values. This fact has been considered in modeling to make sure that proper input matrices are obtained by applying the aforementioned secondary controllers for voltage and frequency.

Some of the control techniques related to the secondary loops are based on communication links, such as the master-slave approach, which can be adapted mostly in systems where inverters are not far from each other.

As described in the background chapter, two control approaches are possible [9]: Single-Master Operation (SMO) and Multi-Master Operation (MMO). The single master operation can be seen in Fig. 3.1. The VSI inverter acts as master and the other inverters operating in PQ modes are slaves. Whenever there is a disturbance, PQ inverters produce a constant set-point and the VSI inverters change their output power based on the frequency error to track the demand. This situation is not desirable as there is still a frequency error that needs to be compensated and also storage devices have a finite storage capacity, which should be dedicated only to the primary load frequency control. The secondary frequency and voltage control can be obtained by changing the nominal set-point values of VSI inverters and also the active and reactive power set-point of PQ inverters according to their rating and capability of producing power. These set-points are the fundamental elements of input matrices. 


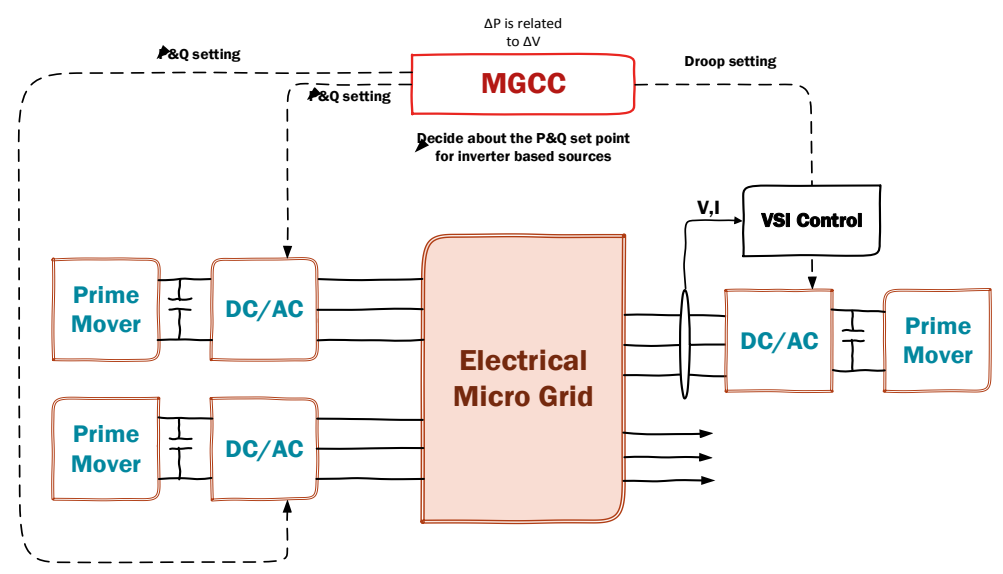

Figure 3.1: Control Scheme for Single Master Operation

\subsection{MODELING OF ISLANDED MiCROGRID}

The modeling approach presented in this chapter divides the system into four modules: VSI inverters, PQ inverters, network and loads. Each inverter is modeled on its individual reference frame and its rotational frequency is set by the local power sharing controller. The VSI inverter model includes the dynamics of current, voltage and power sharing controller, output filter and coupling inductor. While the PQ inverter only models the dynamic of current controller and RL filter. The small-signal flow among the modules is shown in Figure 3.2. Network dynamics are generally neglected in the small-signal modeling of conventional power systems due to the small time constant of the network compared to the rotating machines and their controllers. By contrast, microgrids have inverter-based generating units whose response times are very small so network dynamics can influence the system stability.

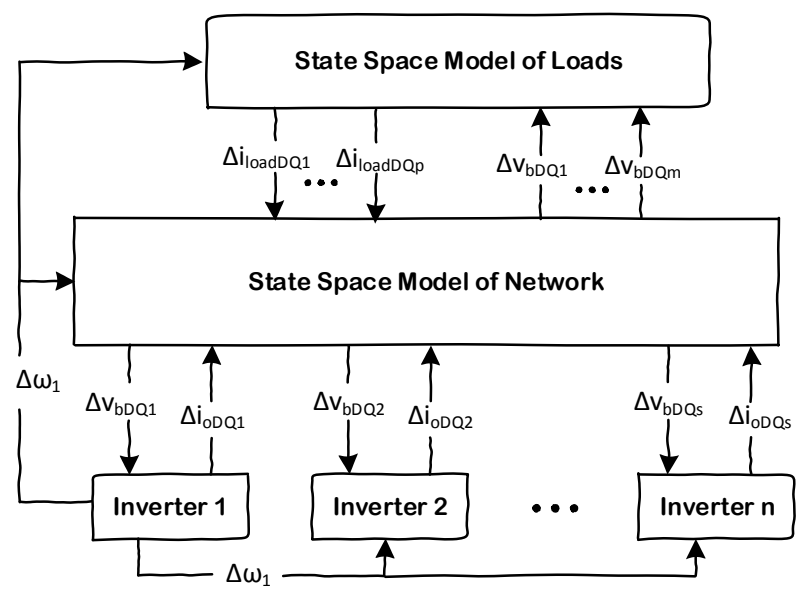

Figure 3.2: Block diagram of small signal state space model related to the microgrid component

Here, the state equations of the network and the loads are represented in the reference frame of one of the individual inverters, which is considered the common reference frame. All the other inverters are translated to this common reference frame using the transformation technique given by equation 3.1. 
In the following equations, $(D-Q)$ is the common reference frame rotating at a frequency of $\omega_{c o m}$, whereas axes $(d-q)_{i}$ are the reference frame of $i$ th inverter rotating at $\omega_{i}$.

$$
\left[f_{D Q}\right]=\left[\begin{array}{cc}
\cos \left(\delta_{i}\right) & -\sin \left(\delta_{i}\right) \\
\sin \left(\delta_{i}\right) & \cos \left(\delta_{i}\right)
\end{array}\right]\left[f_{d q}\right]
$$

In equation 3.1, $\delta_{i}$ is the angle of the reference frame of $i$ th inverter with respect to the common reference frame. In the following sections modeling of all four modules is discussed in more detail. It should be noted that in the following equations, the voltages and currents are described in $d-q$ frame while the other variables such as active and reactive power are scalars.

\subsubsection{MODELING OF VOLTAGE SOURCE INVERTERS (VSI)}

VSI inverters are mostly used to connect distributed generators to the network. Figure 3.3 shows the block diagram of a VSI inverter connected to a microgrid. It has a power electronic section including a three-leg inverter, an output filter and a coupling inductor. Assuming an ideal source from the DG side, the DC bus dynamics can be neglected. There is also a control section comprised of three parts: (1) a power control loop that sets the voltage magnitude and frequency of the fundamental component of the inverter output voltage according to the defined droop characteristics related to the real and reactive powers. (2) The voltage and (3) current controllers. In many papers, the dynamic of the second and third controllers are eliminated for simplification purposes [10]; however, in this work, the detail of these parts are considered in order to have a more accurate model. A state space model is presented for all of the subsystems: control loops, output filter and coupling inductor. The model is constructed in a rotational reference frame set by the power controller of the particular individual inverter.

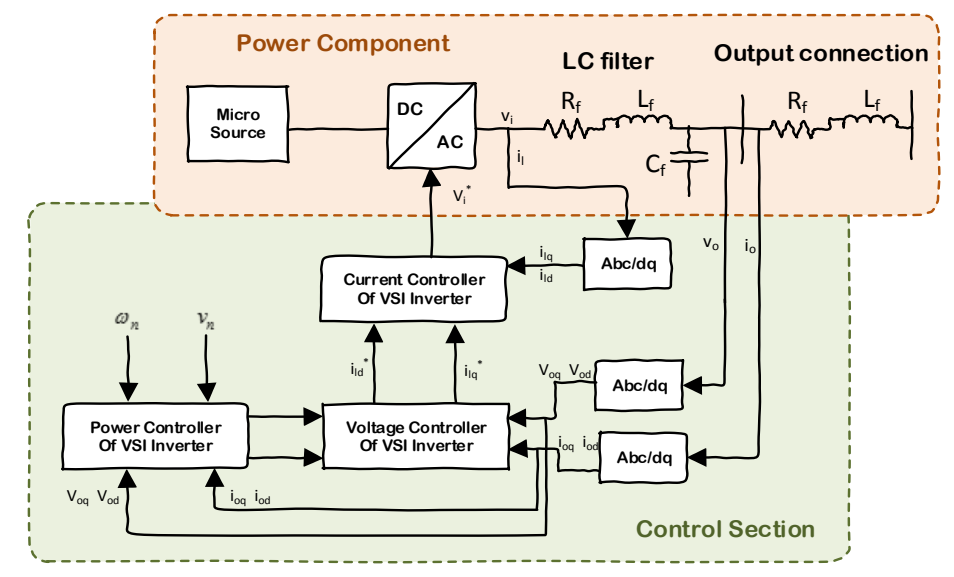

Figure 3.3: Block diagram of VSI inverter 


\section{1) Power Controller:}

The underlying idea behind the droop control is to simulate the governor of a rotating machine. In a conventional power system, rotating machines share any increase in the demand by decreasing the frequency based on their governor droop characteristic. This principle is implemented in VSI inverters by decreasing the reference frequency when there is an increase in the load. Likewise, reactive power is shared by introducing a droop characteristic in the voltage magnitude.

Instantaneous active and reactive power components can be calculated from the measured output voltage and current; afterward, by passing them through low-pass filters (with a cut-off frequency of $\omega_{c o}$ ), the fundamental components, $\mathrm{P}$ and $\mathrm{Q}$, are obtained as equation 3.2-3.3. In these equations, $s$ is the Laplace variable. Finally, the small power changes can be calculated as in $3.4-3.5$.

$$
\begin{aligned}
& p=v_{o d} i_{o d}+v_{o q} i_{o q} \rightarrow P=\frac{\omega_{c o}}{S+\omega_{c o}} p \\
& q=v_{o d} i_{o q}-v_{o q} i_{o d} \rightarrow Q=\frac{\omega_{c o}}{S+\omega_{c o}} q \\
& \Delta \dot{P}=-\omega_{c o} \Delta P+\omega_{c o} \Delta v_{o d} \Delta i_{o d}+\omega_{c o} \Delta v_{o q} \Delta i_{o q} \\
& \Delta \dot{Q}=-\omega_{c o} \Delta Q+\omega_{c o} \Delta v_{o d} \Delta i_{o q}-\omega_{c o} \Delta v_{o q} \Delta i_{o d}
\end{aligned}
$$

The real power sharing between VSIs is attained by using an artificial droop in the inverter's frequency shown in equation 3.6. In the following equations, $\omega_{n}$ is the nominal frequency set-point. The reference frequency, $\omega$, is set according to the droop gain $m$. This will provide artificial inertia characteristics and subsequently will produce a degree of negative feedback.

$$
\omega=\omega_{n}-m P
$$

To share the reactive power among the VSIs, a droop is also introduced in the voltage magnitude as given by equation 3.7. Here, $V_{n}$ stands for the nominal set-point of d-axis output voltage and $l$ defines the voltage droop gain. The control strategy is chosen such that the output reference voltage magnitude is associated with the d-axis of the VSI reference frame, and the q-axis reference value is set to zero.

$$
v_{o d}^{*}=V_{n}-l Q, v_{o q}^{*}=0
$$


As discussed earlier, to construct the complete model on a common reference frame, the reference frame of one of the inverters is taken as the common frame. The variables of each VSI inverter should be expressed in the common reference frame by using an angle for each VSI, given by 3.10. Note that $\delta$ is the angle between each VSI reference frame and the common reference frame.

$$
\begin{aligned}
& \Delta \omega=\Delta \omega_{n}-m \Delta P \\
& \delta=\int\left(\omega-\omega_{\text {com }}\right) \\
& \dot{\delta}=\omega-\omega_{\text {com }} \\
& \dot{\delta}_{2}=\omega_{2}-\omega_{\text {com }}=\left(\omega_{n 2}-m_{2} P_{2}\right)-\left(\omega_{n 1}-m_{1} P_{1}\right)
\end{aligned}
$$

Now, based on 3.11 and 3.12, $\Delta \dot{\delta}$ can be calculated for all VSI inverters. Equations $3.13-3.15$ show this variable for each VSI of a microgrid. The understudied microgrid which is used for modeling in the next chapter has three VSI inverters and $m_{1}, m_{2}$ and $m_{3}$ are considered to be equal for them.

$$
\begin{gathered}
\Delta \dot{\delta}_{1}=\Delta \omega_{1}-\Delta \omega_{\text {com }}=0 \\
\Delta \dot{\delta}_{2}=\Delta \omega_{2}-\Delta \omega_{c o m}=\Delta \omega_{n 2}-\Delta \omega_{n 1}-\left(m_{2} \Delta P_{2}-m_{1} \Delta P_{1}\right) \\
\vdots \\
\Delta \dot{\delta}_{i}=\Delta \omega_{i}-\Delta \omega_{c o m}=\Delta \omega_{n i}-\Delta \omega_{n 1}-\left(m_{i} \Delta P_{i}-m_{1} \Delta P_{1}\right)
\end{gathered}
$$

By changing the nominal frequency set-point of each VSI inverter, $\omega_{n i}$, and considering its variations, $\Delta \omega_{n i}$, as a tool for implementing the secondary frequency control, the secondary control input signal will be obtained by the equations. For example, for the understudied microgrid, $\Delta \omega_{n 1}, \Delta \omega_{n 2}$ and $\Delta \omega_{n 3}$ are used for the secondary frequency control.

The state space model of the power controller is obtained by rearranging equation 3.15 and linearizing equations $3.4-3.5$ and it is finally given in 3.21. The power controller outputs are the variations of output reference voltage $\Delta v^{*}$ and frequency $\Delta \omega$.

Introducing the vector notations of $3.16-3.19$ combines the d-q axis component in the equations. $\Delta i_{l d q}$, $\Delta v_{o d q}, \Delta i_{o d q}$ are the state variables of LC filter and coupling inductance represented in a compact notation by $\Delta x_{f, C I}$ in 3.20 . 


$$
\begin{aligned}
& v_{o d q}^{*}=\left[\begin{array}{c}
v_{o d}^{*} \\
v_{o q}^{*}
\end{array}\right], i_{l d q}=\left[\begin{array}{c}
i_{l d} \\
i_{l q}
\end{array}\right], v_{o d q}=\left[\begin{array}{c}
v_{o d} \\
v_{o q}
\end{array}\right], i_{o d q}=\left[\begin{array}{c}
i_{o d} \\
i_{o q}
\end{array}\right] \\
& {\left[\Delta x_{f, C I}\right]=\left[\begin{array}{c}
\Delta i_{l d q} \\
\Delta v_{o d q} \\
\Delta i_{o d q}
\end{array}\right]} \\
& {\left[\begin{array}{c}
\Delta \dot{\delta} \\
\Delta \dot{P} \\
\Delta \dot{Q}
\end{array}\right]=A_{i}\left[\begin{array}{c}
\Delta \delta \\
\Delta P \\
\Delta Q
\end{array}\right]+B_{i 1}\left[\Delta x_{f, C I}\right]+B_{i 2}\left[\Delta \omega_{c o m}\right]+\left[\begin{array}{l}
1 \\
0 \\
0
\end{array}\right]\left[\Delta \omega_{n}\right]+B_{i 2} \Delta \omega_{n c o m}} \\
& {\left[\begin{array}{c}
\Delta \omega \\
\Delta v^{*}{ }_{\text {odq }}
\end{array}\right]=\left[\begin{array}{c}
C_{i \omega} \\
C_{i v}
\end{array}\right]\left[\begin{array}{c}
\Delta \delta \\
\Delta P \\
\Delta Q
\end{array}\right]+\left[\begin{array}{l}
1 \\
0 \\
0
\end{array}\right]\left[\Delta \omega_{n}\right]} \\
& A_{i}=\left[\begin{array}{ccc}
0 & -m & 0 \\
0 & -\omega_{c o} & 0 \\
0 & 0 & -\omega_{c o}
\end{array}\right], B_{i 2}=\left[\begin{array}{c}
-1 \\
0 \\
0
\end{array}\right] \\
& B_{i 1}=\left[\begin{array}{cccccc}
0 & 0 & 0 & 0 & 0 & 0 \\
0 & 0 & \omega_{c o} I_{o d} & \omega_{c o} I_{o q} & \omega_{c o} V_{o d} & \omega_{c o} V_{o q} \\
0 & 0 & \omega_{c o} I_{o q} & -\omega_{c o} I_{o d} & -\omega_{c o} V_{o q} & \omega_{c o} V_{o d}
\end{array}\right] \\
& C_{i \omega}=\left[\begin{array}{lll}
0 & -m & 0
\end{array}\right], \quad C_{i v}=\left[\begin{array}{ccc}
0 & 0 & -l \\
0 & 0 & 0
\end{array}\right]
\end{aligned}
$$

\section{2) Voltage and Current Controllers:}

Voltage and current controllers of VSI inverters are responsible for providing reference output current, $i^{*}{ }_{\text {odq }}$, and reference input voltage, $v_{i d q}^{*}$ respectively. In Figure 3.4, the block diagram of voltage and current controllers, with standard PI terms, are shown. The state equations of the voltage controller and the current controller are described in 3. 28-3.29 and 3.32-3.33 respectively. Then, the algebraic equations are represented next. 


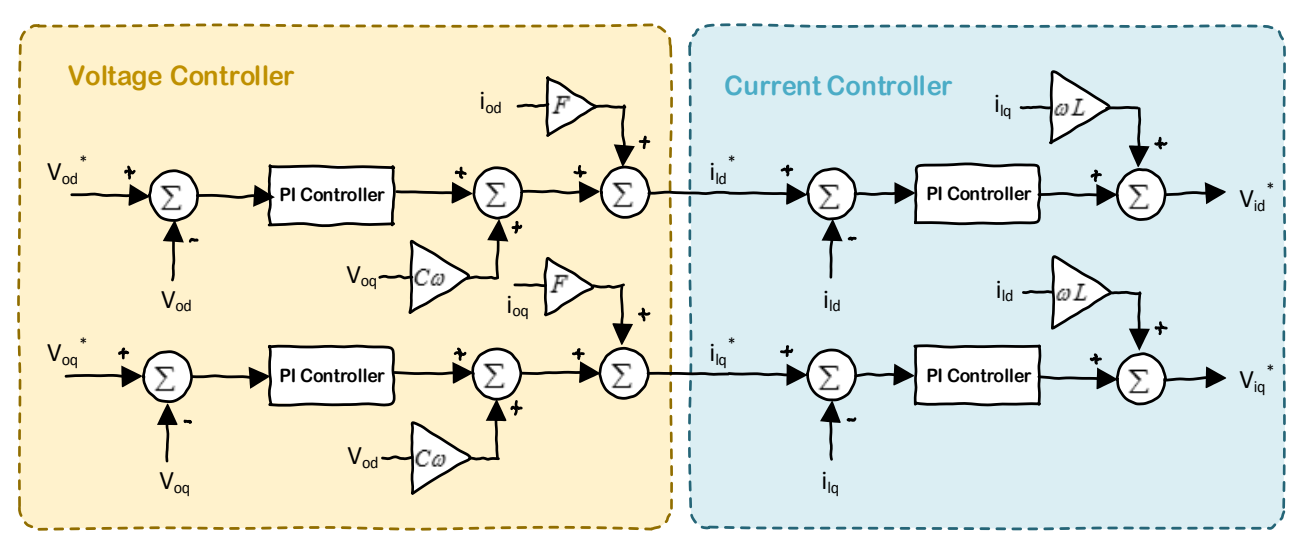

Figure 3.4: Current and voltage controller of VSI inverters

$\frac{d \phi_{d}}{d t}=v_{o d}^{*}-v_{o d}$

$\frac{d \phi_{q}}{d t}=v_{o q}^{*}-v_{o q}$

$i_{l d}^{*}=F i_{o d}-\omega_{n} C_{f} v_{o q}+K_{p v}\left(v_{o d}^{*}-v_{o d}\right)+K_{i v} \phi_{d}$

$i_{l q}^{*}=F i_{o q}+\omega_{n} C_{f} v_{o d}+K_{p v}\left(v_{o q}^{*}-v_{o q}\right)+K_{i v} \phi_{q}$

$\frac{d \gamma_{d}}{d t}=i_{l d}^{*}-i_{l d}$

$\frac{d \gamma_{q}}{d t}=i^{*}{ }_{l q}-i_{l q}$

$v^{*}{ }_{i d}=-\omega_{n} L_{f} i_{l q}+K_{p c}\left(i_{l d}^{*}-i_{l d}\right)+K_{i c} \gamma_{d}$

$v_{i q}^{*}=\omega_{n} L_{f} i_{l d}+K_{p c}\left(i_{l q}^{*}-i_{l q}\right)+K_{i c} \gamma_{q}$

Here, the input to the subsystems is split into two terms: the reference input and the feedback inputs. Equations 3.36 - 3.37 show the states and output of voltage controller and in $3.38-3.39$ those related to current controller are demonstrated. 


$$
\begin{aligned}
& \int\left[\Delta \dot{\phi}_{d q}\right]=[0]\left[\Delta \phi_{d q}\right]+B_{v 1}\left[\Delta v_{o d q}^{*}\right]+B_{v 2}\left[\Delta x_{f, C I}\right] \\
& \left\{\left[\Delta i_{l d q}^{*}\right]=C_{v}\left[\Delta \phi_{d q}\right]+D_{v 1}\left[\Delta v_{o d q}^{*}\right]+D_{v 2}\left[\Delta x_{f, C I}\right]\right. \\
& \left\{\left[\Delta \dot{\gamma}_{d q}\right]=[0]\left[\Delta \gamma_{d q}\right]+B_{c 1}\left[\Delta i_{l d q}^{*}\right]+B_{c 2}\left[\Delta x_{f, C I}\right]\right. \\
& \left\{\left[\Delta v^{*}{ }_{i d q}\right]=C_{c}\left[\Delta \gamma_{d q}\right]+D_{c 1}\left[\Delta i^{*}{ }_{l d q}\right]+D_{c 2}\left[\Delta x_{f, C I}\right]\right.
\end{aligned}
$$

$\varphi_{d q}$ and $\gamma_{d q}$ are auxiliary state variables, which measure the variation of voltage and current $\left(v_{o d q}, i_{l d q}\right)$ from the reference values. $v_{i}$ and $i_{i}$ are the voltage and current of DC/AC inverter, $v_{o}$ is the voltage measured after LC filter and $i_{o}$ is the current of coupling inductance. These variables are depicted in Figure 3.3.

$k_{p v}$ and $k_{i v}$ are the parameters of PI inside the voltage controller and $k_{p c}$ and $k_{i c}$ are those related to the current controller PI. $R_{f}, C_{f}$ and $L_{f}$ are also parameters of LC filter.

$$
\begin{aligned}
& \Delta \phi_{d q}=\left[\begin{array}{c}
\Delta \phi_{d} \\
\Delta \phi_{q}
\end{array}\right] \\
& B_{v 1}=\left[\begin{array}{ll}
1 & 0 \\
0 & 1
\end{array}\right], B_{v 2}=\left[\begin{array}{cccccc}
0 & 0 & -1 & 0 & 0 & 0 \\
0 & 0 & 0 & -1 & 0 & 0
\end{array}\right] \\
& C_{v}=\left[\begin{array}{cc}
K_{i v} & 0 \\
0 & K_{i v}
\end{array}\right], D_{v 1}=\left[\begin{array}{cc}
K_{p v} & 0 \\
0 & K_{p v}
\end{array}\right] \\
& D_{v 2}=\left[\begin{array}{cccccc}
0 & 0 & -K_{p v} & -\omega_{n} C_{f} & F & 0 \\
0 & 0 & \omega_{n} C_{f} & -K_{p v} & 0 & F
\end{array}\right] \\
& \Delta \gamma_{d q}=\left[\begin{array}{c}
\Delta \gamma_{d} \\
\Delta \gamma_{q}
\end{array}\right], \quad B_{c 1}=\left[\begin{array}{ll}
1 & 0 \\
0 & 1
\end{array}\right] \\
& B_{c 2}=\left[\begin{array}{cccccc}
-1 & 0 & 0 & 0 & 0 & 0 \\
0 & -1 & 0 & 0 & 0 & 0
\end{array}\right] \\
& C_{c}=\left[\begin{array}{cc}
K_{i c} & 0 \\
0 & K_{i c}
\end{array}\right], \quad D_{c 1}=\left[\begin{array}{cc}
K_{p c} & 0 \\
0 & K_{p c}
\end{array}\right] \\
& D_{c 2}=\left[\begin{array}{cccccc}
-K_{p c} & -\omega_{n} L_{f} & 0 & 0 & 0 & 0 \\
\omega_{n} L_{f} & -K_{p c} & 0 & 0 & 0 & 0
\end{array}\right]
\end{aligned}
$$




\section{3) Output LC Filter and Coupling Inductance:}

The state space model for the output filter and the coupling inductance, shown in 3.58, can be formed by use of equations $3.52-3.57$ considering that the VSI can provide the reference voltage.

$$
\begin{aligned}
& \frac{d i_{l d}}{d t}=\frac{-r_{f}}{L_{f}} i_{l d}+\omega i_{l q}+\frac{1}{L_{f}} v_{i d}-\frac{1}{L_{f}} v_{o d} \\
& \frac{d i_{l q}}{d t}=\frac{-r_{f}}{L_{f}} i_{l q}-\omega i_{l d}+\frac{1}{L_{f}} v_{i q}-\frac{1}{L_{f}} v_{o q} \\
& \frac{d v_{o d}}{d t}=\omega v_{o q}+\frac{1}{C_{f}} i_{l d}-\frac{1}{C_{f}} i_{o d} \\
& \frac{d v_{o q}}{d t}=-\omega v_{o d}+\frac{1}{C_{f}} i_{l q}-\frac{1}{C_{f}} i_{o q} \\
& \frac{d i_{o d}}{d t}=\frac{-r_{c}}{L_{c}} i_{o d}+\omega i_{o q}+\frac{1}{L_{c}} v_{o d}-\frac{1}{L_{c}} v_{b d} \\
& \frac{d i_{o q}}{d t}=\frac{-r_{c}}{L_{c}} i_{o q}-\omega i_{o d}+\frac{1}{L_{c}} v_{o q}-\frac{1}{L_{c}} v_{b q} \\
& {\left[\begin{array}{l}
\Delta i_{l d q} \\
\Delta \dot{v}_{o d q} \\
\Delta i_{o d q}
\end{array}\right]=A_{l}\left[\begin{array}{l}
\Delta i_{l d q} \\
\Delta v_{o d q} \\
\Delta i_{o d q}
\end{array}\right]+B_{l 1}\left[\Delta v_{i d q}\right]+B_{l 2}\left[\Delta v_{b d q}\right]+} \\
& B_{l 3}\left[\Delta \omega_{c o m}\right]+B_{l 3}\left[\Delta \omega_{n c o m}\right]
\end{aligned}
$$

$\Delta \omega_{n c o m}=\Delta \omega_{n 1}$ 


$$
\begin{aligned}
A_{l} & =\left[\begin{array}{cccccc}
\frac{-r_{f}}{L_{f}} & \omega_{0} & \frac{-1}{L_{f}} & 0 & 0 & 0 \\
-\omega_{0} & \frac{-r_{f}}{L_{f}} & 0 & \frac{-1}{L_{f}} & 0 & 0 \\
\frac{1}{C_{f}} & 0 & 0 & \omega_{0} & -\frac{1}{C_{f}} & 0 \\
0 & \frac{1}{C_{f}} & -\omega_{0} & 0 & 0 & -\frac{1}{C_{f}} \\
0 & 0 & \frac{1}{C_{f}} & 0 & \frac{-r_{c}}{L_{c}} & \omega_{0} \\
0 & 0 & 0 & \frac{1}{L_{c}} & -\omega_{0} & \frac{-r_{c}}{L_{c}}
\end{array}\right] \\
B_{l 1} & =\left[\begin{array}{cc}
\frac{1}{L_{f}} & 0 \\
0 & \frac{1}{L_{f}} \\
0 & 0 \\
0 & 0 \\
0 & 0 \\
0 & 0
\end{array}\right] \quad B_{l 2}=\left[\begin{array}{cc}
0 & 0 \\
0 & 0 \\
0 & 0 \\
0 & 0 \\
\frac{-1}{L_{c}} & 0 \\
0 & \frac{-1}{L_{c}}
\end{array}\right] \quad B_{l 3}=\left[\begin{array}{c}
I_{l q} \\
-I_{l d} \\
V_{o q} \\
-V_{o d} \\
I_{o q} \\
-I_{o d}
\end{array}\right]
\end{aligned}
$$

\section{4) Complete Model of VSI Inverters:}

In order to connect the VSI inverters to a microgrid, all output currents and voltages need to be expressed in a common reference frame. So, here the $\Delta i_{o d q}$ will be converted to the $\Delta i_{o D Q}$ by use of the transformation technique given in equation 3.1. Finally, after merging all previous state equations, the model of one VSI inverter will be organized as in 3.64. After building the state space model for each VSI inverter, they can be added up to form a unit model for all the VSI inverters. Since the understudied microgrid that is going to be used during the next chapter has three VSIs, the corresponding VSI state space model will be obtained as shown in $3.65-3.66$.

$$
\left[\Delta \dot{x}_{V S I i}\right]=A_{V S I i}\left[\Delta x_{V S I i}\right]+B_{V S I i}\left[\Delta v_{b D Q}\right]+B_{i \omega c o m}\left[\Delta \omega_{c o m}\right]+\left[\begin{array}{c}
\Delta \omega_{n i}-\Delta \omega_{n c o m} \\
0 \\
B_{l 3-i} \Delta \omega_{n c o m}
\end{array}\right]
$$




$$
\begin{gathered}
\left\{\begin{array}{c}
{\left[\Delta \dot{x}_{V S I}\right]=A_{V S I}\left[\Delta x_{I N V}\right]+B_{V S I}\left[\Delta v_{b D Q}\right]+B_{c o m}\left[\Delta \omega_{c o m}\right]+B_{n}\left[\Delta \omega_{n}\right]} \\
{\left[\Delta i_{o D Q}\right]=C_{V S I}\left[\Delta x_{V S I}\right]}
\end{array}\right. \\
B_{n}=\left[\begin{array}{ccc}
1-1 & 0 & 0 \\
\vdots & \vdots & \vdots \\
B_{l c l 3-1} & 0 & 0 \\
-1 & 1 & 0 \\
\vdots & \vdots & \vdots \\
B_{l c l 3-2} & 0 & 0 \\
-1 & 0 & 1 \\
\vdots & \vdots & \vdots \\
B_{l c l 3-3} & 0 & 0
\end{array}\right],\left[\Delta \omega_{n}\right]=\left[\begin{array}{c}
\Delta \omega_{n 1} \\
\Delta \omega_{n 2} \\
\Delta \omega_{n 3}
\end{array}\right]
\end{gathered}
$$

\subsubsection{MODELING OF PQ OR CURRENT SOURCE INVERTER (CSI)}

CSI (PQ) inverters are those inverters who receive the specific set-point about active and reactive powers. As they are not responsible for the frequency and voltage regulations, their state space has only four state variables because of only one current controller instead of three controllers for VSIs. These four state variables are shown in 3.69

$$
X_{P Q}=\left[\begin{array}{llll}
\gamma_{d} & \gamma_{q} & i_{l d} & i_{l q}
\end{array}\right]^{T}
$$

$\gamma_{d}$ and $\gamma_{q}$ are the auxiliary state variables used for considering the error of PQ inverter currents $\left(i_{l d}, i_{l q}\right)$ from the reference values. Figure 3.5 shows the block diagram related to the current controller of a PQ inverter. The current controller adjusts the $d$-q terms of output current $i_{l}$. The term $\alpha$ in this figure is defined so that the quadrature term of output voltage reaches zero.

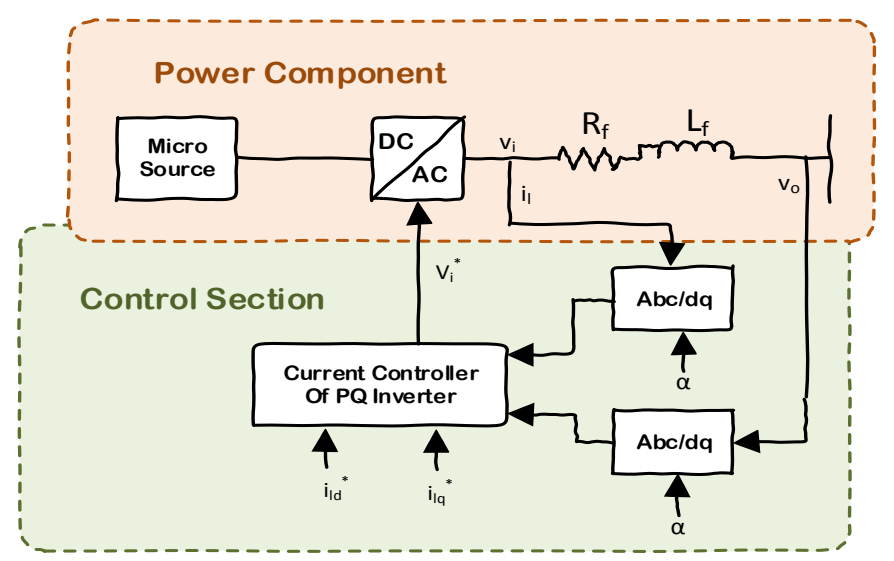

Figure 3.5: Current controller of $P Q$ inverters 
Two state variables of PQ inverters are related to the dynamics of the current controller block. These equations are described in $3.70-3.71$. In fact, these state variables are auxiliary variables that illustrate the behavior of PI controllers used inside the current controller block.

$$
\begin{aligned}
& \frac{d \gamma_{d}}{d t}=i_{l d}^{*}-i_{l d} \\
& \frac{d \gamma_{q}}{d t}=i_{l q}^{*}-i_{l q} \\
& v_{i d}^{*}=v_{o d}-\omega_{n} L_{f} i_{l q}+K_{p c}\left(i_{l d}^{*}-i_{l d}\right)+K_{i c} \gamma_{d} \\
& v^{*}{ }_{i q}=v_{o d}+\omega_{n} L_{f} i_{l d}+K_{p c}\left(i_{l q}^{*}-i_{l q}\right)+K_{i c} \gamma_{q} \\
& \left\{\begin{array}{l}
{\left[\Delta \dot{\gamma}_{d q}\right]=[0]\left[\Delta \gamma_{d q}\right]+B_{1}\left[\Delta i^{*}{ }_{l d q}\right]+B_{2}\left[\Delta i_{l d q}\right]} \\
{\left[\Delta v^{*}{ }_{i d q}\right]=C_{1}\left[\Delta \gamma_{d q}\right]+D_{1}\left[\Delta i^{*}{ }_{l d q}\right]+D_{2}\left[\Delta i_{l d q}\right]+D_{3}\left[\Delta v_{o d q}\right]}
\end{array}\right. \\
& \Delta \gamma_{d q}=\left[\begin{array}{l}
\Delta \gamma_{d} \\
\Delta \gamma_{q}
\end{array}\right], \quad B_{1}=\left[\begin{array}{ll}
1 & 0 \\
0 & 1
\end{array}\right], B_{2}=\left[\begin{array}{cc}
-1 & 0 \\
0 & -1
\end{array}\right] \\
& C_{1}=\left[\begin{array}{cc}
K_{i c} & 0 \\
0 & K_{i c}
\end{array}\right], \quad D_{1}=\left[\begin{array}{cc}
K_{p c} & 0 \\
0 & K_{p c}
\end{array}\right] \\
& D_{2}=\left[\begin{array}{cc}
-K_{p c} & -\omega_{n} L_{f} \\
\omega_{n} L_{f} & -K_{p c}
\end{array}\right], D_{3}=\left[\begin{array}{ll}
1 & 0 \\
0 & 1
\end{array}\right]
\end{aligned}
$$

Equations $3.83-3.84$ calculate the active and reactive power. As previously mentioned $\alpha$ is set so that $v_{\text {oq }}$ becomes zero. According to this, the $\mathrm{P}$ and $\mathrm{Q}$ equation will be simplified as below:

$$
\begin{aligned}
& \left.\begin{array}{l}
P=v_{o d} i_{l d}+v_{o q} i_{l q} \\
Q=v_{o q} i_{l d}-v_{o d} i_{l q}
\end{array}\right\} \rightarrow A s \quad v_{o q}=0 \Rightarrow\left\{\begin{array}{c}
P=v_{o d} i_{l d} \\
Q=-v_{o d} i_{l q}
\end{array}\right.
\end{aligned}
$$

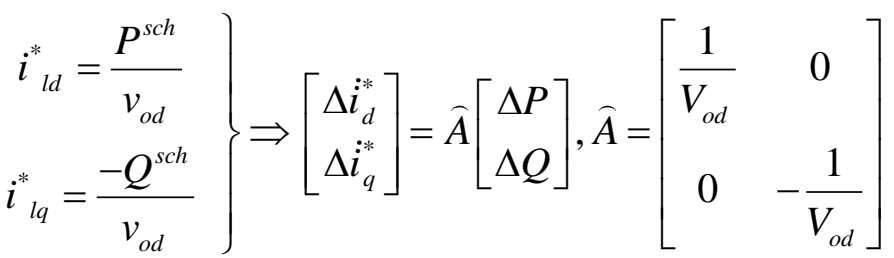


The other two state variable of the PQ inverter are related to the dynamics of the RL filter. The change in $d-q$ term of output current that is fed to the microgrid through the RL filter are shown in $3.88-3.89$, which form the remaining state variables.

$$
\begin{aligned}
& \frac{d i_{l d}}{d t}=\frac{-r_{f}}{L_{f}} i_{l d}+\omega_{c o m} i_{l q}+\frac{1}{L_{f}} v_{i d}-\frac{1}{L_{f}} v_{o d} \\
& \frac{d i_{l q}}{d t}=\frac{-r_{f}}{L_{f}} i_{l q}-\omega_{c o m} i_{l d}+\frac{1}{L_{f}} v_{i q}-\frac{1}{L_{f}} v_{o q}
\end{aligned}
$$

Equation 3.88 - 3.89 can be rewritten to shape the state space form used for small signal dynamic model, shown in $3.90-3.91$.

It can be seen that the current state variables are related to different parameters, which some of them were defined in 3.75; by substituting them, the total model for the PQ inverter will be achieved in 3.96. By transforming the variable to the reference frame, the final model will be obtained as in $3.101-3.102$.

$$
\begin{aligned}
& {\left[\Delta \dot{i}_{l d q}\right]=\bar{A}_{l}\left[\Delta i_{l d q}\right]+\bar{B}_{l 1}\left[\Delta v_{i d q}\right]+\bar{B}_{l 2}\left[\Delta v_{b d q}\right]} \\
& +\bar{B}_{l 3}\left[\Delta \omega_{\text {com }}\right]+\bar{B}_{l 3}\left[\Delta \omega_{\text {ncom }}\right] \\
& \Delta \omega_{n c o m}=\Delta \omega_{n 1} \\
& \bar{A}_{l}=\left[\begin{array}{cc}
\frac{-r_{f}}{L_{f}} & \omega_{0} \\
-\omega_{0} & \frac{-r_{f}}{L_{f}}
\end{array}\right], \quad \bar{B}_{l 1}=\left[\begin{array}{cc}
\frac{1}{L_{f}} & 0 \\
0 & \frac{1}{L_{f}}
\end{array}\right] \\
& \bar{B}_{l 2}=\left[\begin{array}{cc}
\frac{-1}{L_{f}} & 0 \\
0 & \frac{-1}{L_{f}}
\end{array}\right], \quad \bar{B}_{l 3}=\left[\begin{array}{c}
I_{l q} \\
-I_{l d}
\end{array}\right]
\end{aligned}
$$

There is an assumption here: the $\Delta \delta$ is constant for PQ inverters. 


$$
\begin{aligned}
& {\left[\begin{array}{c}
\Delta \dot{\gamma}_{d q} \\
\Delta \dot{i}_{l d q}
\end{array}\right]=\bar{A}\left[\begin{array}{c}
\Delta \gamma_{d q} \\
\Delta i_{l d q}
\end{array}\right]+\bar{B}_{1}\left[\Delta v_{o d q}\right]+\bar{B}_{2}\left[\begin{array}{c}
\Delta P_{I n} \\
\Delta Q_{I n}
\end{array}\right]} \\
& +\bar{B}_{3}\left[\omega_{\text {com }}\right]+\bar{B}_{3}\left[\Delta \omega_{\text {ncom }}\right] \\
& \bar{A}=\left[\begin{array}{cc}
0 & B_{2} \\
\bar{B}_{l 1} C_{1} & \bar{A}_{l}+\bar{B}_{l 1} D_{2}
\end{array}\right], \bar{B}_{2}=\left[\begin{array}{c}
B_{1} \widehat{A} \\
\bar{B}_{l 1} D_{1} \widehat{A}
\end{array}\right] \\
& \bar{B}_{1}=\left[\begin{array}{c}
B_{1} \\
\bar{B}_{l 1}\left(D_{1}+D_{3}\right)+\bar{B}_{l 2}
\end{array}\right], \bar{B}_{3}=\left[\begin{array}{c}
0 \\
\bar{B}_{l 3}
\end{array}\right] \\
& \left\{\begin{aligned}
{\left[\Delta \dot{x}_{P Q}\right]=\bar{A}\left[\Delta x_{P Q}\right]+} & \bar{B}_{11}\left[\Delta v_{o D Q}\right]+\bar{B}_{2}\left[\begin{array}{c}
\Delta P_{I n} \\
\Delta Q_{I n}
\end{array}\right] \\
& +\bar{B}_{3}\left[\omega_{c o m}\right]+\bar{B}_{3}\left[\Delta \omega_{n c o m}\right] \\
{\left[\Delta i_{o D Q}\right]=\bar{C}\left[\Delta x_{P Q}\right] } &
\end{aligned}\right. \\
& \bar{B}_{11}=\bar{B}_{1}\left[T_{s}^{-1}\right] \\
& \bar{C}=\left[\begin{array}{lll}
0 & 0 & T_{s}
\end{array}\right]
\end{aligned}
$$

\subsubsection{MODELING OF NETWORK AND LOAD}

Considering a typical RL load and line modeled by a series impedance, their state equations are given in 3.105 - 3.106. In [23], more detail about the small-signal state-space model of loads and lines are discussed.

$$
\begin{aligned}
& {\left[\Delta \dot{x}_{\text {Net }}\right]=A_{N}\left[\Delta x_{N e t}\right]+B_{1 N}\left[\Delta v_{b D Q}\right]+B_{2 N}\left[\Delta \omega_{c o m}\right]+B_{2 N}\left[\Delta \omega_{n c o m}\right]} \\
& {\left[\Delta \dot{x}_{\text {Load }}\right]=A_{L}\left[\Delta x_{\text {Load }}\right]+B_{1 L}\left[\Delta v_{b D Q}\right]+B_{2 L d}\left[\Delta \omega_{c o m}\right]+B_{2 L}\left[\Delta \omega_{\text {ncom }}\right]}
\end{aligned}
$$

\subsubsection{MICROGRID MODEL}

The microgrid model is obtained by combining the models of the 4 modules given above by equations 3.65, 3.101, 3.105 and 3.106. A short summary of each element equation is brought here. 


$$
\begin{gathered}
\left\{\begin{array}{c}
{\left[\Delta \dot{x}_{V S I}\right]=A_{V S I}\left[\Delta x_{V S I}\right]+B_{V S I}\left[\Delta v_{b D Q}\right]+B_{c o m}\left[\Delta \omega_{c o m}\right]+B_{n}\left[\Delta \omega_{n}\right]} \\
{\left[\Delta i_{o D Q}\right]=C_{V S I}\left[\Delta x_{V S I}\right]}
\end{array}\right. \\
\left\{\begin{array}{c}
{\left[\Delta \dot{x}_{P Q}\right]=\bar{A}\left[\Delta x_{P Q}\right]+\bar{B}_{11}\left[\Delta v_{o D Q}\right]+\bar{B}_{2}\left[\begin{array}{c}
\Delta P_{I n} \\
\Delta Q_{I n}
\end{array}\right]+\bar{B}_{3}\left[\omega_{c o m}\right]+\bar{B}_{3}\left[\Delta \omega_{n c o m}\right]} \\
{\left[\Delta i_{o D Q}\right]=\bar{C}\left[\Delta x_{P Q}\right]}
\end{array}\right. \\
{\left[\Delta x_{N e t}\right]=A_{N}\left[\Delta x_{N e t}\right]+B_{1 N}\left[\Delta v_{b D Q}\right]+B_{2 N}\left[\Delta \omega_{c o m}\right]+B_{2 N}\left[\Delta \omega_{n c o m}\right]} \\
{\left[\Delta \dot{x}_{\text {Load }}\right]=A_{L}\left[\Delta x_{L o a d}\right]+B_{1 L}\left[\Delta v_{b D Q}\right]+B_{2 L}\left[\Delta \omega_{c o m}\right]+B_{2 L}\left[\Delta \omega_{n c o m}\right]}
\end{gathered}
$$

Microgrid model is given in the form of:

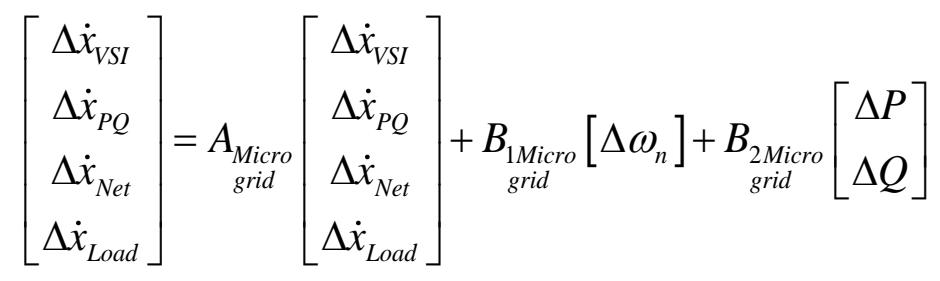

$\mathrm{A}_{\text {microgrid }}, \mathrm{B}_{1 \text { Microgrid }}$ and $\mathrm{B}_{2 \mathrm{Microgrid}}$ matrices can completely describe the microgrid. The obtained model has appropriate input terms that allows it to work on the secondary frequency control and power management of the microgrid.

It can be seen that the $\Delta \mathrm{V}_{\mathrm{bDQ}}$ vector is needed for all four equations $3.107,3.109,3.111$, and 3.112 because it is inputted for the subsystem. This node voltage vector is defined by considering a virtual resister between each node and ground.

$$
\left[\Delta \dot{v}_{b D Q}\right]=R_{N}\left(M_{V S I}\left[\Delta i_{o D Q}\right]+M_{P Q}\left[\Delta i_{P Q o D Q}\right]+M_{N}\left[\Delta i_{\text {LineDQ }}\right]+M_{L}\left[\Delta i_{\text {LoadDQ }}\right]\right)
$$

$\mathrm{M}_{\mathrm{VSI}}, \mathrm{M}_{\mathrm{PQ}}, \mathrm{M}_{\mathrm{N}}$ and $\mathrm{M}_{\mathrm{L}}$ are defined based on the configuration of the system. Eventually, the Microgrid matrices are as $3.115-3.117$. 


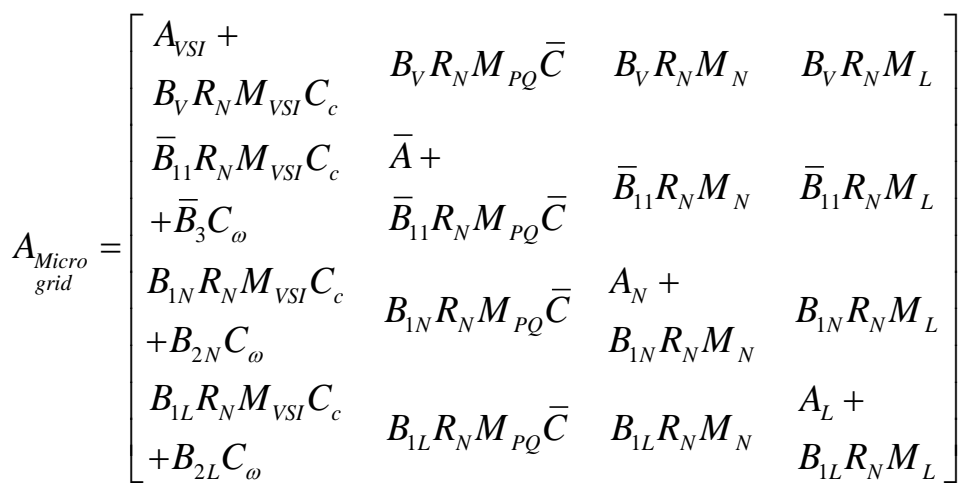

$$
\begin{aligned}
& B_{\text {gricro }}=\left[\begin{array}{ccc}
B_{n} & \\
\bar{B}_{3} & 0 & 0 \\
B_{2 N} & 0 & 0 \\
B_{2 L} & 0 & 0
\end{array}\right], B_{\text {gricro }}=\left[\begin{array}{c}
0 \\
\bar{B}_{2} \\
0 \\
0
\end{array}\right]
\end{aligned}
$$




\section{Chapter 4}

\section{Load Frequency Control Analysis of Islanded Microgrid}

\subsection{Primary and Secondary Frequency Control Concepts}

In an islanded microgrid, a coordinated active power regulation is essential for having active power management. Especially, an autonomous power control strategy is needed in order to ensure robustness of microgrid in the case of failure of any communication link. Droop control strategy using frequency error of each unit to distribute active power is an acceptable solution to this requisite. However, an important point is that a coordinated active power control strategy should consider the status of all microgrid elements, the available power of generating units and demand of power consumption. If there are any energy storage devices, the state of charge (SOC) must also be fully taken into account.

Microgrids have different kinds of DGs with their own particular functionality in the load frequency control. Based on the participation of DG's in the primary frequency control algorithm, they are divided into two categories of VSI and PQ inverters. During the primary control loop, the VSI inverters are those that act 
like a rotating machine and control the voltage and frequency, while the PQ inverters only produce a certain amount of active and reactive power. The PQ inverters will just act in the secondary control loop by changing their set-point and providing additional requested power for the microgrid. The VSI inverters themselves consist of two different categories. All of them are helpful in primary control but a group of them used as storage devices are not supposed to have any role in secondary control. The reason behind that is the limited power they can produce long-term. Table 4.1 shows how DG's are categorized based on their contribution in primary and secondary load frequency control:

TABLE 4.1

DG TYPES AND THEIR CONTRIBUTION DURING COORDINATED CONTROL

\begin{tabular}{lccc}
\hline \hline \multicolumn{2}{c}{ DG TYPE } & $\begin{array}{c}\text { Primary } \\
\text { Control }\end{array}$ & $\begin{array}{c}\text { Secondary } \\
\text { Control }\end{array}$ \\
\hline \multirow{2}{*}{ VSI inverters } & Generating units & $\mathbf{X}$ & $\mathbf{X}$ \\
& $\begin{array}{c}\text { Energy Storage } \\
\text { Devices }\end{array}$ & $\mathbf{X}$ & \\
CSI (PQ) inverters & & & $\mathbf{X}$ \\
\hline
\end{tabular}

Communication links make the system more expensive and less reliable. Also, in a typical microgrid, inverters can be far away from each other, making a communication link less attractive. In this chapter, it is shown that the communication of DGs is very limited. In other words, the communication between VSI inverters are eliminated and only PQ inverters are supposed to communicate. In fact, the secondary control for VSI inverters is a decentralized approach, which means that there is no need for communication in order to coordinate the VSI inverters; however, having PQ inverters in the microgrid means communication between VSI and PQ inverters is necessary to send the PQ signal for these inverters. These communication links are optimally chosen to have as little communication as possible. Proposed control techniques are based on local measurements using minimum or no communication at all when feasible.

As discussed earlier, controlling the frequency of microgrid has two level of primary and secondary control. In the primary control, the nominal frequency set-point, $\omega_{n i}$, is fixed and any change in the load will cause a change in $\omega$ of the inverter based on the defined droop for power controller. Then, in the secondary control, if the inverter is connected to a generating unit, $\omega_{n i}$ will be changed in a way that the frequency is brought back to the nominal value and if it is connected to a storage device, $\omega_{n i}$ will remain fixed as before. The reason is that these elements only participate in primary frequency control (because their inverters are working as VSI inverters) and since they should not be considered as a long-term power producer, they cannot contribute to secondary controls like other VSI inverters. Figure 4.1 shows how these two categories of VSI inverters act during the two levels of frequency control. 

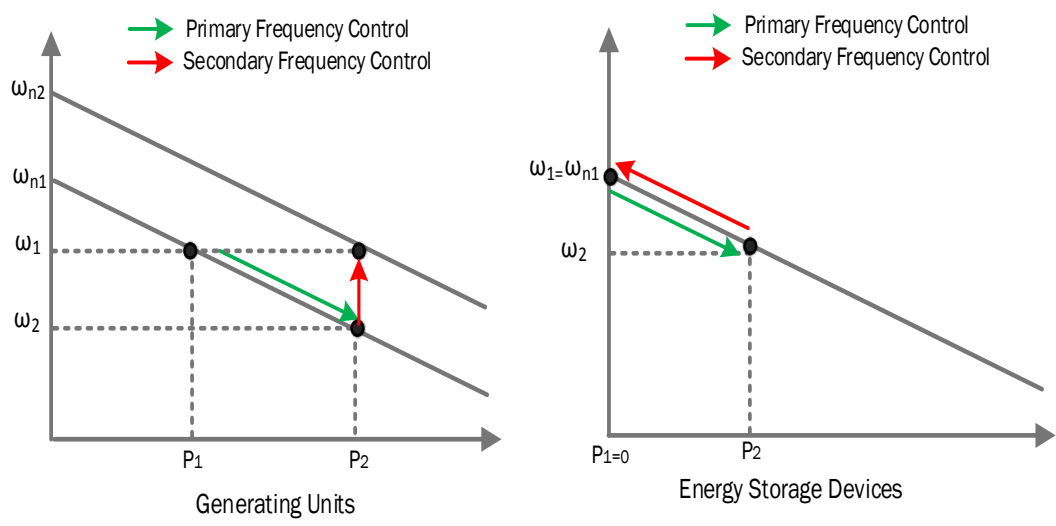

Figure 4.1: Secondary control for VSI inverters-generating units and energy storage devices

Based on Figure 4.1, it can be seen that $\omega_{\text {ni }}$ will change for all VSI inverters except those who are connected to the storage devices. These devices should come back to the nominal frequency point without any change in the $\omega_{\mathrm{ni}}$ of their curves.

It should be noticed that, usually, the droop defined in the primary control is related to the rating of each DGs, but in the secondary control, the criteria can be changed to consider other parameters in steady-state situations. In other words, each DG has a participating factor that can be a function of the speed of response or cost of producing power or any other criteria or it can remain the same as before.

Microgrids also consist of PQ inverters that do not have droop curves. They measure the frequency by phase lock loop (PLL) and their $\omega$ for the inverter is calculated based on the measured frequency. These elements should receive PQ set-point. One way is using communication network and the other solution can be bus signaling.

\subsection{IMPLEMENTATION AND ANALYSIS OF SECONDARY FREQUENCY CONTROL}

\subsubsection{MICROGRID TEST SYSTEM}

Using the procedure discussed in chapter 3, the state space matrices can be formed for any microgrid with different number of VSI and PQ inverters along with the loads and the network. However, to have the numerical values of the matrices, the system parameters along with the steady state values of the operating point is required. So, first of all, a load flow analysis is performed, (using MATLAB software in this study) to obtain the required values. Then, the obtained state space model can be used for both stability analysis and small signal transient analysis. The eigenvalues of A matrix are used for investigating the stability issues. It should be noticed that for different cases (with different operating points), the A matrix differs and the eigenvalues will lie in various locations which are going to be discussed in more detail in stability analysis section. Figure 4.2 shows the steps of these investigation procedure in which MATLAB 
environment is being used to conduct it. For small signal transient analysis, a state space block model is used in Simulink. The calculated A and B matrices along with C and D are provided for the mentioned block. Three inputs are defined for the microgrid where the first one is related to the nominal set-points of frequency, second one provides the P-Q set-points of PQ inverters and finally the last one provides a proper input to simulate the load change as a disturbance for the system. The input related to VSI inverters $\left(\Delta \omega_{n i}\right)$ is formed by receiving the feedback from the outputs $\left(\omega_{i}\right)$, comparing it with $60 \mathrm{~Hz}$ and giving the error to a PI controller at the end to set the $\Delta \omega_{n i}$ for all three VSIs. This procedure is also shown in Figure 4.3.

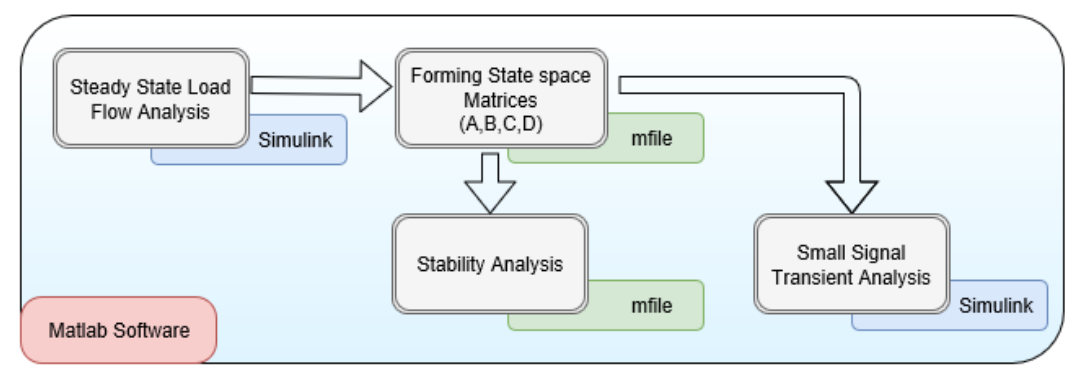

Figure 4.2: Steps of simulation and analysis procedure along with the implementation environments

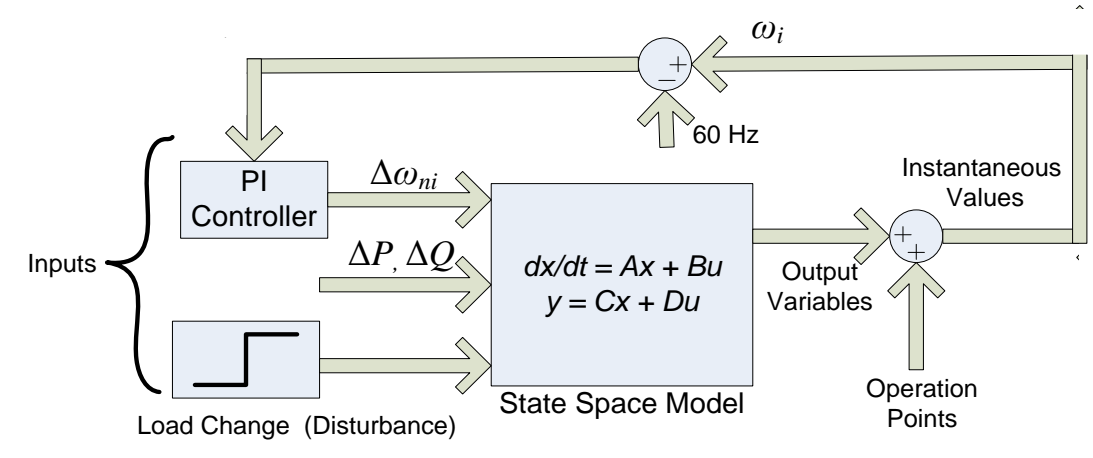

Figure 4.3: Secondary control implementation in MATLAB/Simulink

Here in this study, an isolated microgrid with three VSIs and one PQ inverter is used to demonstrate the validity of secondary frequency control and the effect of different parameters such as PQ inverter contribution on the stability margin of microgrid. Figure 4.4 depicts the single-line diagram of the case study. The nominal voltage and frequency are $380 \mathrm{~V}$ and $60 \mathrm{~Hz}$. The microgrid parameters are summarized in Table 4.2. All inverters are assumed to have the same rating, consequently all of them have similar droop gain. The operating point parameters in different cases are outlined in Tables 4.3 to 4.7. Table 4.3 and 4.4 show the load flow results when the PQ inverter is not connected to the microgrid and the other three tables show the operating point values when PQ is attached. 


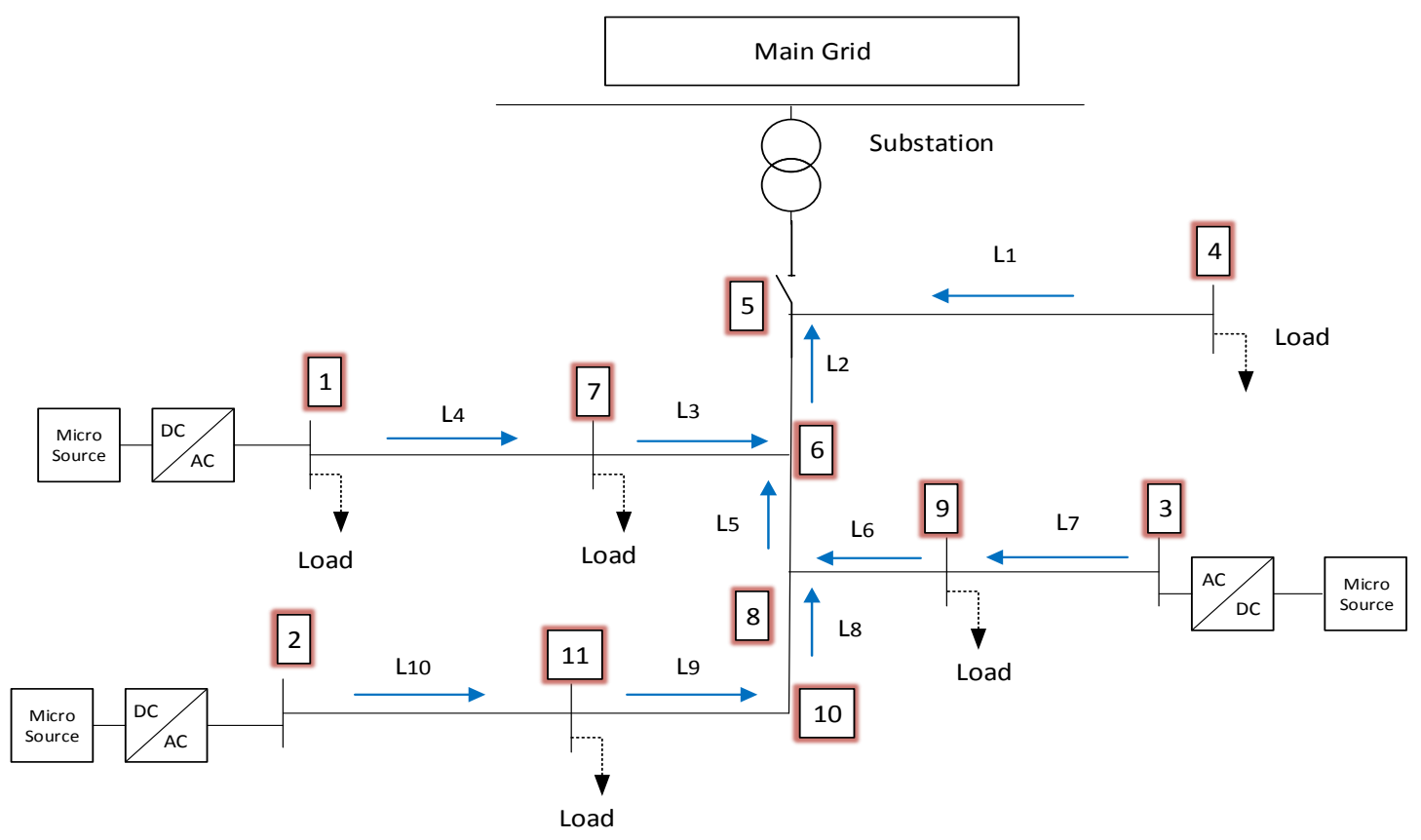

Figure 4.4: Microgrid case study

TABLE 4.2: STUDY SYSTEM PARAMETERS [23]

\begin{tabular}{ll|lc|ll|ll}
\multicolumn{6}{l}{ Inverters Parameters } \\
\hline $\mathrm{r}_{\mathrm{f}}$ & $0.1 \Omega$ & $\mathrm{L}_{\mathrm{c}}$ & $0.35 \mathrm{mH}$ & $\mathrm{K}_{\mathrm{pc}}$ & 10.5 & $\mathrm{~m}$ & $9.4 \mathrm{e}-5$ \\
\hline $\mathrm{L}_{\mathrm{f}}$ & $1.35 \mathrm{mH}$ & $\omega_{\mathrm{co}}$ & 31.41 & $\mathrm{~K}_{\mathrm{ic}}$ & $16 \mathrm{e} 3$ & 1 & $1.3 \mathrm{e}-3$ \\
\hline $\mathrm{C}_{\mathrm{f}}$ & $50 \mu \mathrm{F}$ & $\mathrm{r}_{\mathrm{c}}$ & $0.03 \Omega$ & $\mathrm{K}_{\mathrm{pv}}$ & 0.05 & $\mathrm{~K}_{\mathrm{iv}}$ & 390 \\
\hline \hline
\end{tabular}

TABLE 4.3

\begin{tabular}{c|c|c|c|c|}
\hline \multicolumn{5}{|c|}{ VSI INVERTERS STEADY STATE LOAD FLOW VALUES (NO PQ INVERTER IS ADDED) } \\
\hline \multirow{2}{*}{ VSI\#1 } & {$\left[\mathrm{I}_{\mathrm{od}}, \mathrm{I}_{\mathrm{oq}}\right]$} & {$\left[\mathrm{I}_{\mathrm{ld}}, \mathrm{I}_{\mathrm{lq}}\right]$} & {$\left[\mathrm{V}_{\mathrm{od}}, \mathrm{V}_{\mathrm{oq}}\right]$} & {$\left[\mathrm{V}_{\mathrm{bd}}, \mathrm{V}_{\mathrm{bq}}\right]$} \\
\hline VSI\#2 & {$[7.559,-4.441]$} & {$[7.559,4.359]$} & {$[381.41,0]$} & {$[372,-93.88]$} \\
\hline VSI\#3 & {$[7.538,-4.056]$} & {$[7.538,4.714]$} & {$[381.49,0]$} & {$[377.57,-93.69]$} \\
\hline \hline
\end{tabular}

TABLE 4.4

\begin{tabular}{c|c|c|c|c|c}
\hline \hline \multicolumn{7}{|c|}{ LiNE'S STEADY STATE LOAD FLOW VALUES (NO PQ INVERTER IS ADDED) } \\
\hline & {$\left[\mathrm{I}_{\text {Lined }}, \mathrm{I}_{\text {lineq }}\right]$} & & {$\left[\mathrm{I}_{\text {Lined }}, \mathrm{I}_{\text {lineq }}\right]$} & & {$\left[\mathrm{I}_{\text {Lined }}, \mathrm{I}_{\text {lineq }}\right]$} \\
\hline Line1,2 & {$[3.33,-2.86]$} & Line 5 & {$[5.26,-3.01]$} & Line 8 & {$[3.17,-1.47]$} \\
\hline Line 3 & {$[-1.93,0.15]$} & Line 6 & {$[2.09,-1.54]$} & Line 9 & {$[3.17,-1.47]$} \\
\hline Line 4 & {$[4.16,-2.47]$} & Line 7 & {$[7.55,-4.14]$} & Line 10 & {$[7.54,-4.06]$} \\
\hline \hline
\end{tabular}


TABLE 4.5

\begin{tabular}{c|c|c|c|c}
\hline \hline \multicolumn{5}{|c|}{ VSI AND PQ INVERTERS STEADY STATE LOAD FLOW VALUES (PQ INVERTER IS ADDED) } \\
\hline & {$\left[\mathrm{I}_{\mathrm{od}}, \mathrm{I}_{\mathrm{oq}}\right]$} & {$\left[\mathrm{I}_{\mathrm{ld}}, \mathrm{I}_{\mathrm{lq}}\right]$} & {$\left[\mathrm{V}_{\mathrm{od}}, \mathrm{V}_{\mathrm{oq}}\right]$} & {$\left[\mathrm{V}_{\mathrm{bd}}, \mathrm{V}_{\mathrm{bq}}\right]$} \\
\hline VSI\#1 & {$[6.281,-3.09]$} & {$[6.281,5.682]$} & {$[381.49,0]$} & {$[377.5,-90.8]$} \\
\hline VSI\#2 & {$[6.261,-3.045]$} & {$[6.261,5.727]$} & {$[381.49,0]$} & {$[378.68,-90.52]$} \\
\hline VSI\#3 & {$[6.277,-3.092]$} & {$[6.277,5.679]$} & {$[381.49,0]$} & {$[377.49,-90.72]$} \\
\hline PQ & {$[5.09,-2.61]$} & ---- & --- & {$[377.5,-90.8]$} \\
\hline \hline
\end{tabular}

TABLE 4.6

\begin{tabular}{c|c|c|c|c|c|}
\hline \hline \multicolumn{7}{|c|}{ LINE'S STEADY STATE LOAD FLOW VALUES (PQ INVERTER IS ADDED) } \\
\hline & {$\left[\mathrm{I}_{\text {Lined }}, \mathrm{I}_{\text {lineq }}\right]$} & & {$\left[\mathrm{I}_{\text {Lined }}, \mathrm{I}_{\text {lineq }}\right]$} & & {$\left[\mathrm{I}_{\text {Lined }}, \mathrm{I}_{\text {lineq }}\right]$} \\
\hline Line1,2 & {$[3.56,-2.77]$} & Line 5 & {$[2.23,-1.30]$} & Line 8 & {$[1.67,-0.61]$} \\
\hline Line 3 & {$[1.33,-1.47]$} & Line 6 & {$[0.56,-0.69]$} & Line 9 & {$[1.67,-0.61]$} \\
\hline Line 4 & {$[7.73,-3.86]$} & Line 7 & {$[6.28,-3.09]$} & Line 10 & {$[6.26,-3.04]$} \\
\hline \hline
\end{tabular}

TABLE 4.7

\begin{tabular}{c|c|c|c|c|c|}
\hline \hline \multicolumn{7}{|c|}{ LOADING CONDITION OF THE MICROGRID } \\
\hline & Bus 1 & Bus 4 & Bus 7 & Bus 9 & Bus 11 \\
\hline Active load (kW) & 2.8 & 3 & 5 & 4.5 & 3.5 \\
\hline Reactive load (kVAR) & 0.8 & 1.5 & 0.8 & 0.9 & \\
\hline \hline
\end{tabular}

\subsubsection{STABILITY ANALYSIS}

The eigenvalues of the state space model are depicted in Figure 4.5 with and without PQ inverter's involvement in supplying the demand. The green crosses show the system's eigenvalues when the PQ inverter is not attached to bus 1 and the blue crosses show the eigenvalues when it is connected to the microgrid providing $25 \%$ of the total load. The communication link is assumed to be perfect without any loss or delay. It is noted that during the higher demand, the low-frequency modes drift toward locations that decrease the stability margin. This fact is described in Figure 4.6 and 4.7. Here, by assigning a portion of the demand to the PQ inverter, the dominant modes of the power sharing dynamics move toward a more stable region. So, participation of the PQ inverter improves the stability margin.

As mentioned, adding active and reactive load to the microgrid will affect the location of the eigenvalues. Even though, the trend of relocating the eigenvalues are different, they both push the most dominant eigenvalues towards the right-hand side of real axis, which means the stability margin of the microgrid is being reduced when either active or reactive load is increased. This fact is shown in Figure 4.6 and 4.7 for active and reactive load increase, respectively. 


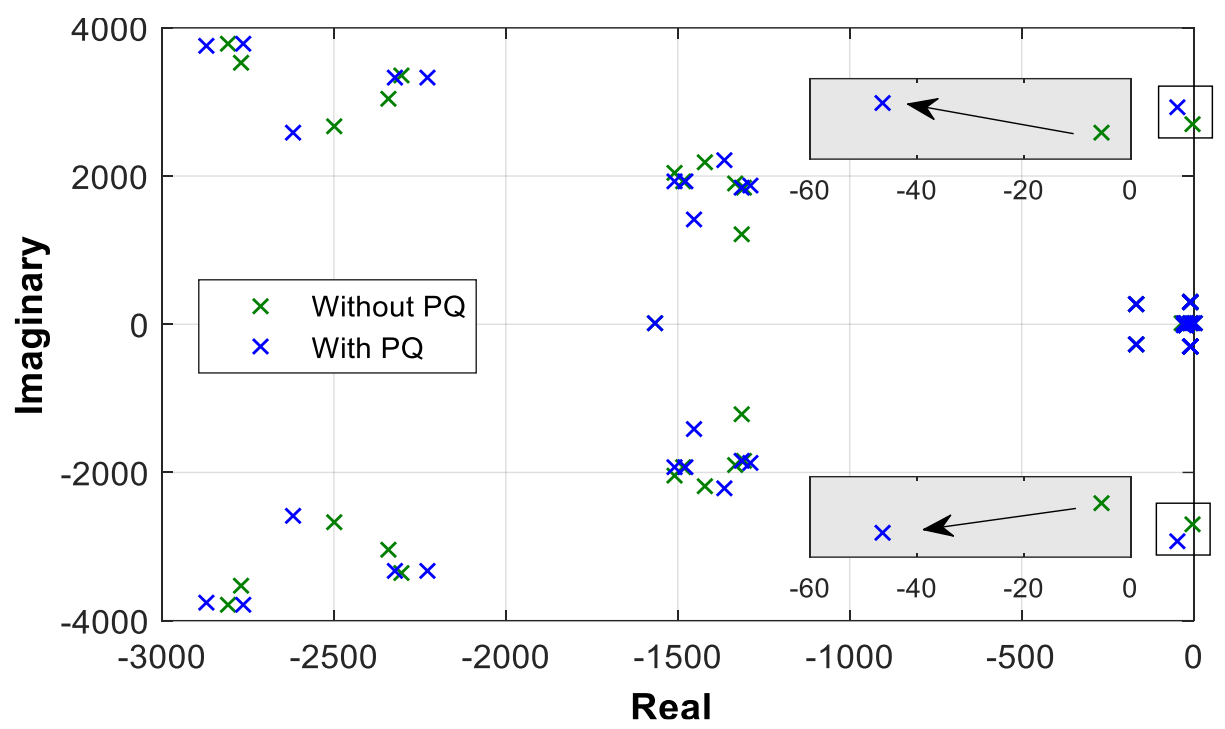

Figure 4.5: Eigenvalues of system with and without $P Q$ inverter

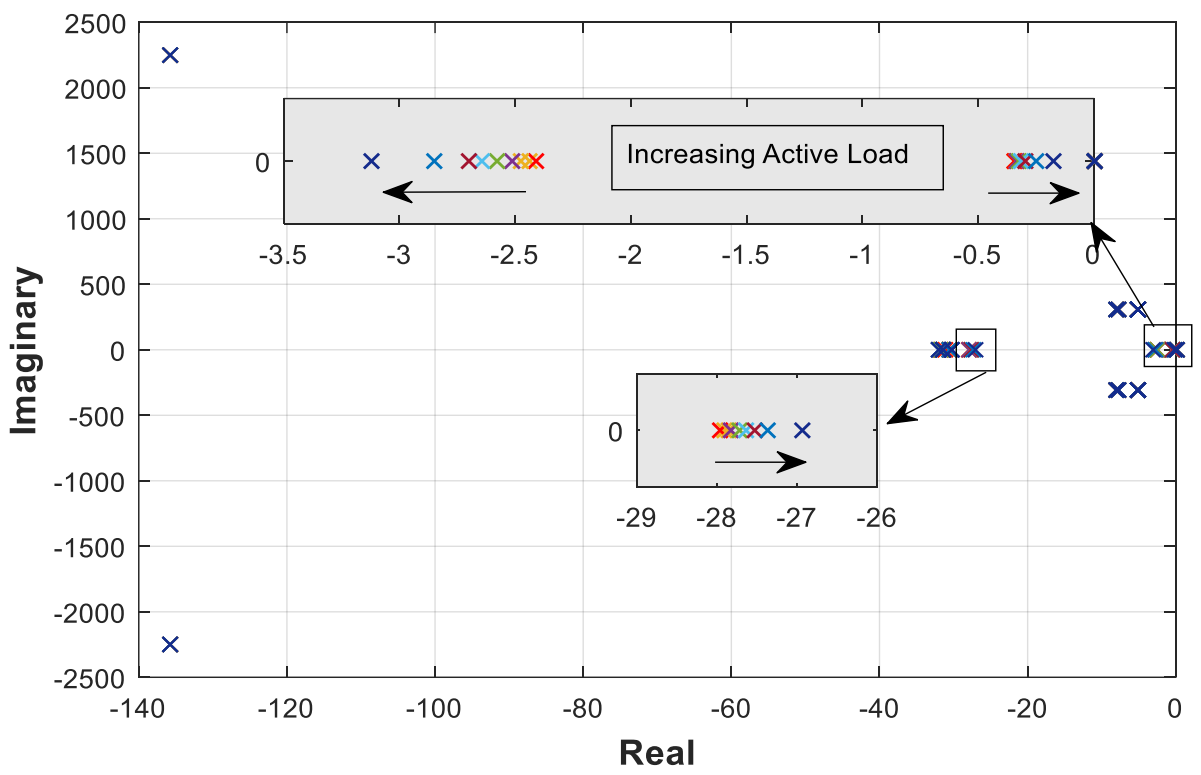

Figure 4.6: Eigenvalue's relocation trend by increasing the active load at bus 11 


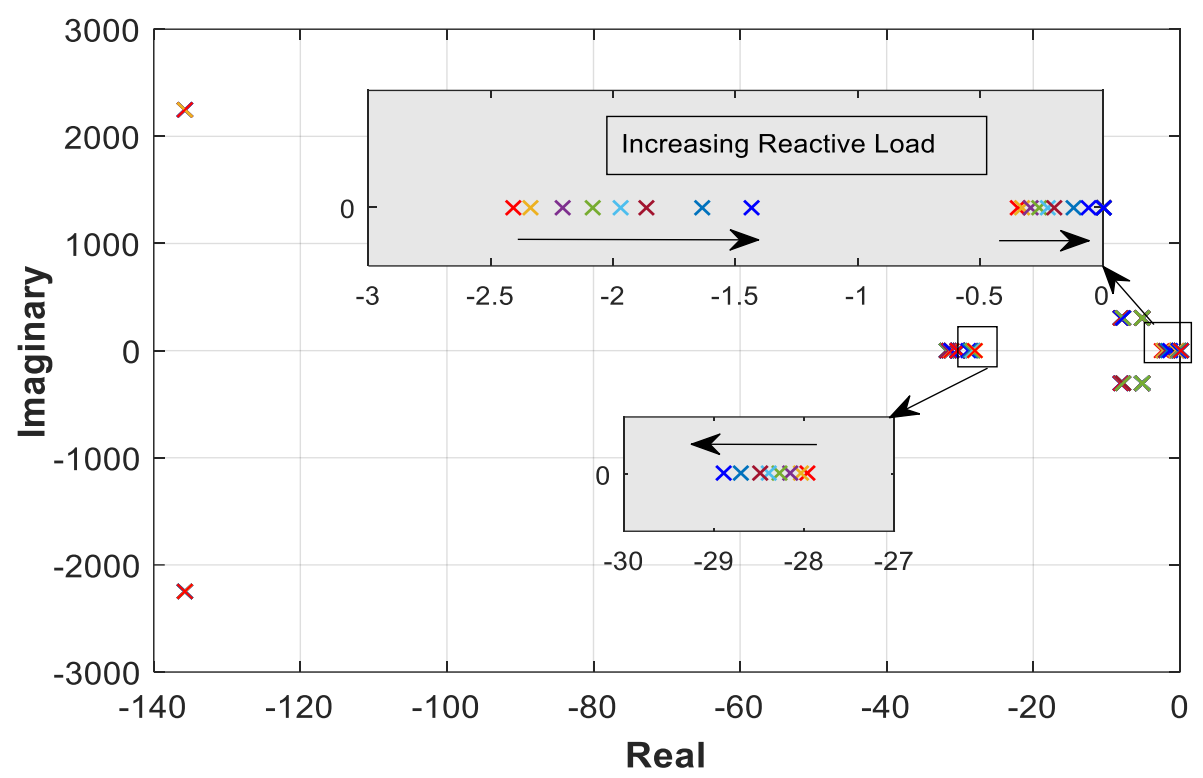

Figure 4.7: Eigenvalue's relocation trend by increasing the reactive load at bus 11

Finally, it is also important to notice the PQ inverters loading condition as well. As it was discussed earlier, adding PQ inverters to the microgrid can improve the stability of the system. But it is also important to see how different participation factors, defined for PQ inverter, can influence the stability dynamics. Figure 4.8 is showing where the eigenvalues lie when the participation factor is changing from $10 \%$ to $20 \%$ of the total demand. As it could be expected, the figure is denoting that having a higher participation factor will bring the dominant eigenvalues to a more stable region.

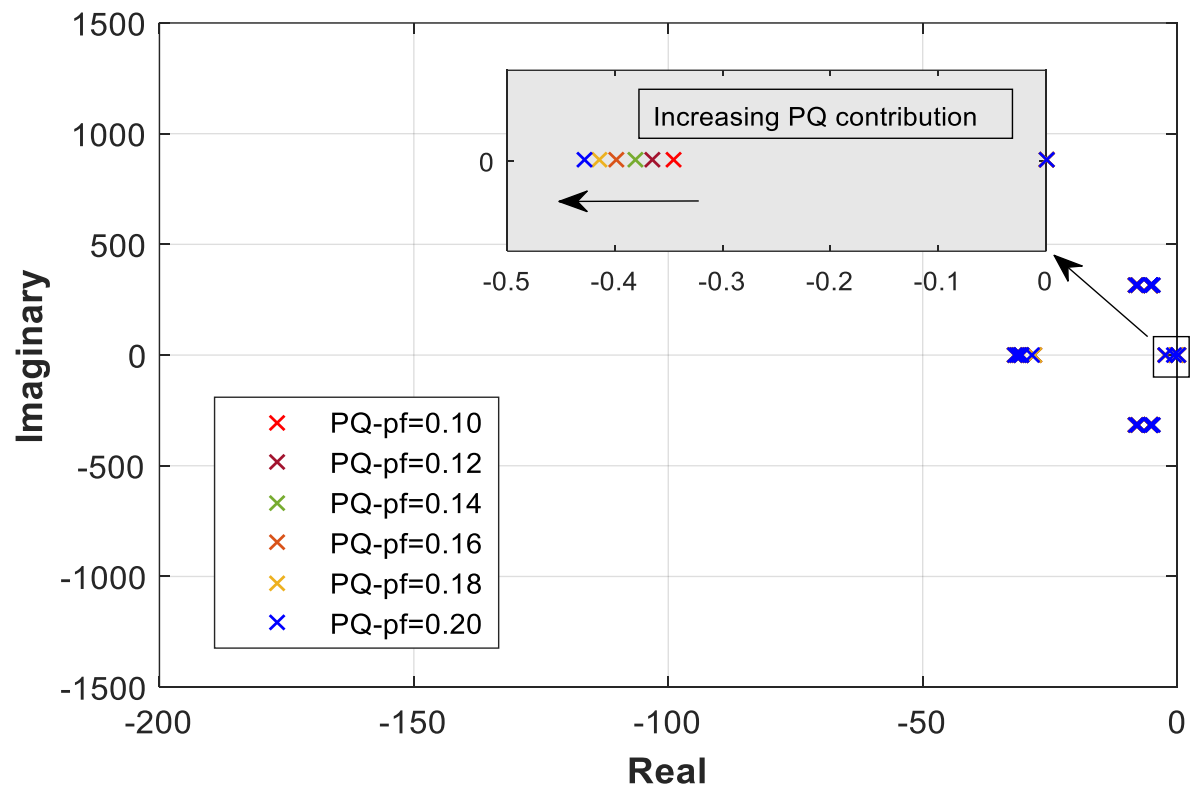

Figure 4.8: Eigenvalue's relocation trend by increasing the $P Q$ inverter's participation factor 


\subsubsection{SMall Signal Transient ANALYSIS}

The simulation results are presented for three different scenarios. In the first and second cases, there is no PQ inverter in the microgrid. The PQ inverter will be added to the microgrid during the third case. The only difference between first and second case is in the VSI inverter used inside the microgrid. In the first case, all three inverters of microgrid are generating unit VSIs, which means they all participate in both primary and secondary frequency control but in the second case, one of the generating units is substituted by a storage device to see how the frequency control strategy will be changed. In all cases, it has been assumed that there is a sudden load change in bus 11 .

\section{1) Case 1}

In the first case, all DG's will act the same and they will change their $\omega_{n i}$ similarly because the $m$ is equal for all three DGs. In other words, they will act in primary and secondary control with the same participation factor of 0.33 .

Figure 4.9 can completely show the way secondary control is traced on all three inverters. Before the load change, the microgrid frequency is $\omega_{1}$, considered to be the nominal frequency, and then it has been decreased to $\omega_{2}$ because of the load increase. Finally, by changing $\omega_{n i}$, the frequency will be brought back to $\omega_{1}$.

\section{2) Case 2}

In the second scenario, two DGs are considered as generating unit VSI inverters and the third one is ESS. This means that the participation factor in primary control is 0.33 but in secondary control, this value will change. Table 4.8 shows how the participation factor will change in the second case.

The secondary control for two generating unit-VSIs and one storage device-VSI are illustrated in figure 4.10. It can be seen in this figure that the $\Delta \omega_{n i}$ for the third inverter (inverter connected to energy storage device) is equal to zero.

TABLE 4.8

\begin{tabular}{cccc} 
DG TYPES AND THEIR PARTICIPATION FACTOR DURING COORDINATED CONTROL IN SECOND & DG Type & \multicolumn{2}{c}{ Participation factor } \\
\cline { 3 - 4 } & & Primary Control & $\begin{array}{c}\text { Secondary } \\
\text { Control }\end{array}$ \\
\hline \hline DG 1 & Generating Unit & 0.33 & 0.5 \\
DG 2 & Generating Unit & 0.33 & 0.5 \\
DG 3 & Energy Storage & 0.33 & 0 \\
\hline
\end{tabular}




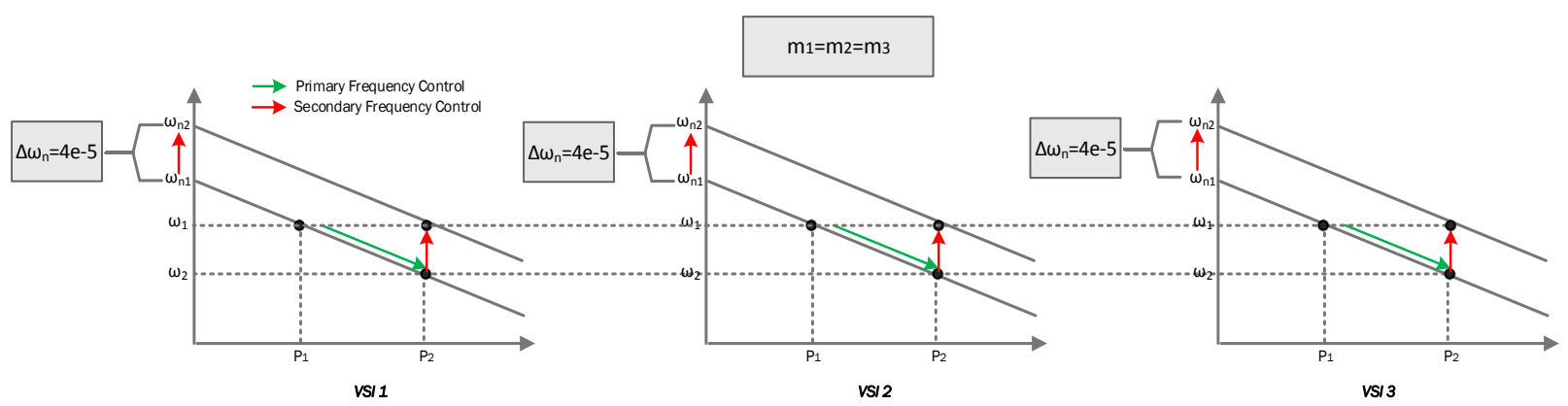

Figure 4.9: Case 1-Secondary control for VSI 1,2 and 3 (all of them are generating units)

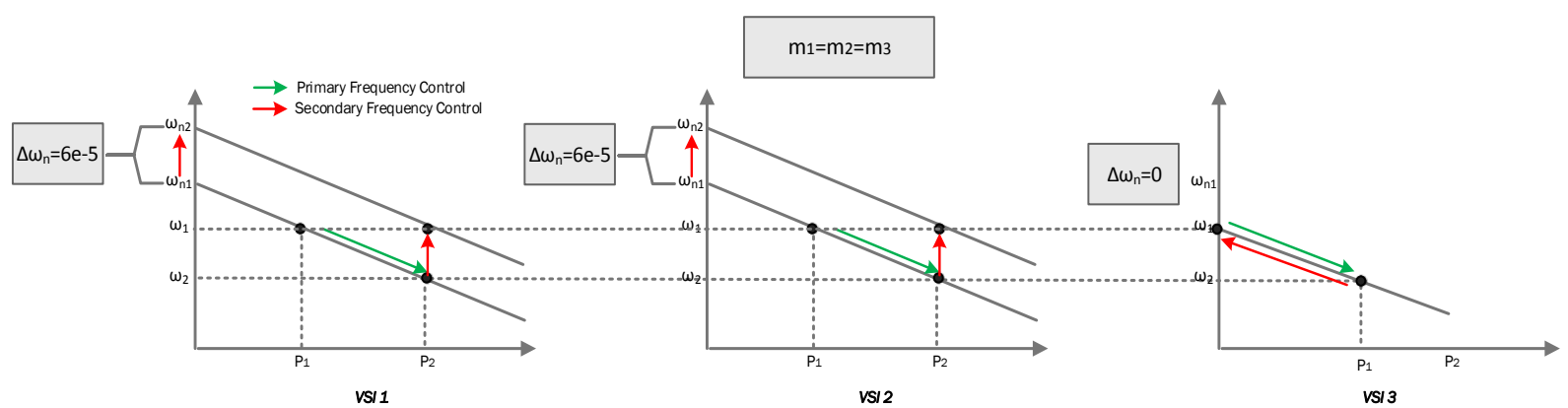

Figure 4.10: Case 2-Secondary control for VSI 1,2 and 3 (1 and 2 are generating units and 3 is energy storage device)

The result of the simulation for case 1 and 2 are depicted in Figures 4.11-4.14. Figure 4.11 compares the output power of inverters in cases 1 and 2. The broken lines are the output power of inverters related to case 1 and the solid lines are associated with case 2. By examining the red curve, relevant to the third inverter, it can be found out that in the second case, this inverter only contributes in transient situations, which means it supports the system exclusively in primary control, not the secondary one.

Figure 4.12 shows the change in nominal frequency set-point $\left(\Delta \omega_{n i}\right)$ in both cases. It clarifies that in the second case, the generating units need to increase their nominal frequency set-points more than the first case because of the more participation they require to have.

Figure 4.13 illustrates the reference frequency changes of inverters. Because the load change happens in bus 11 and the second inverter is the nearest inverter to this bus, its frequency is more affected than the others.

Finally, Figure 4.14 shows the trace of delta for all three inverters in both scenarios. Obviously, only the delta of the third inverter will change in the second scenario. 


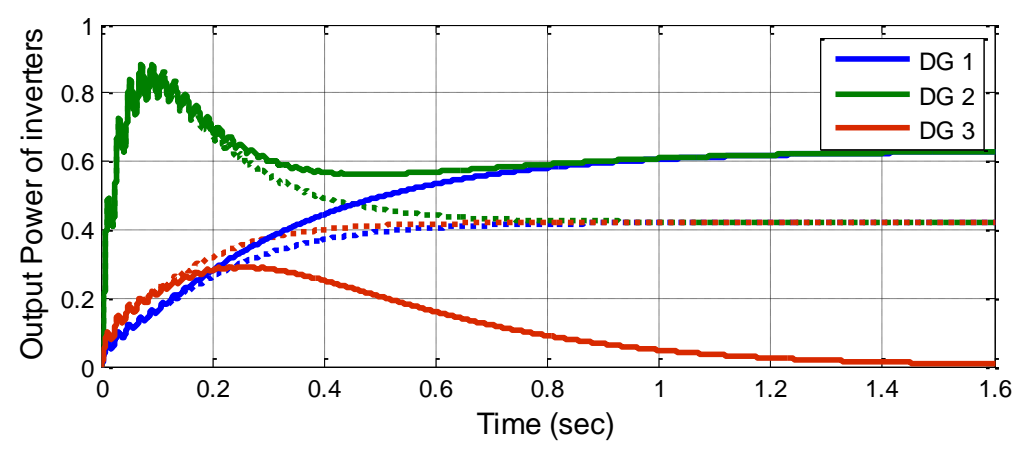

Figure 4.11: Output power changes of inverters in case 1 and 2. (Solid lines: case 1, Broken lines: Case 2)

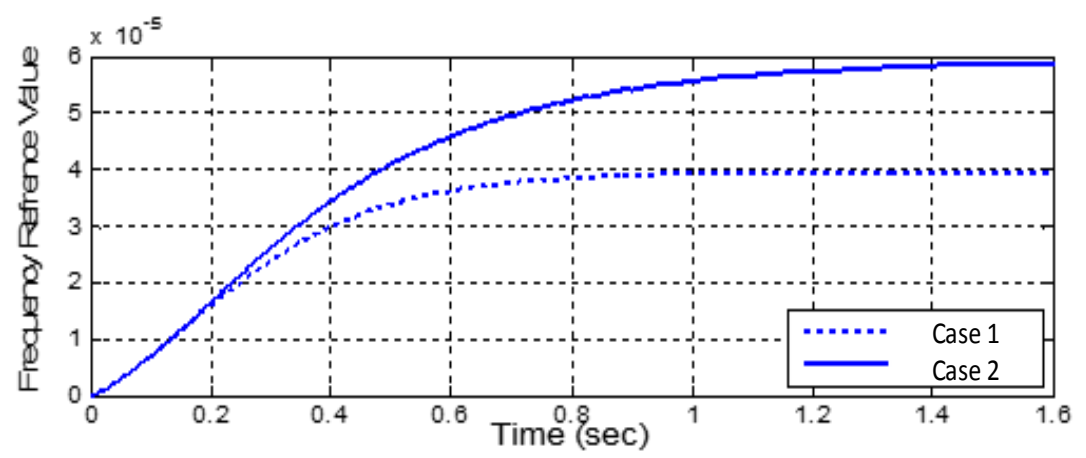

Figure 4.12: Change in nominal frequency set-points in case 1 and 2. (Solid lines: case 1, Broken lines: Case 2)

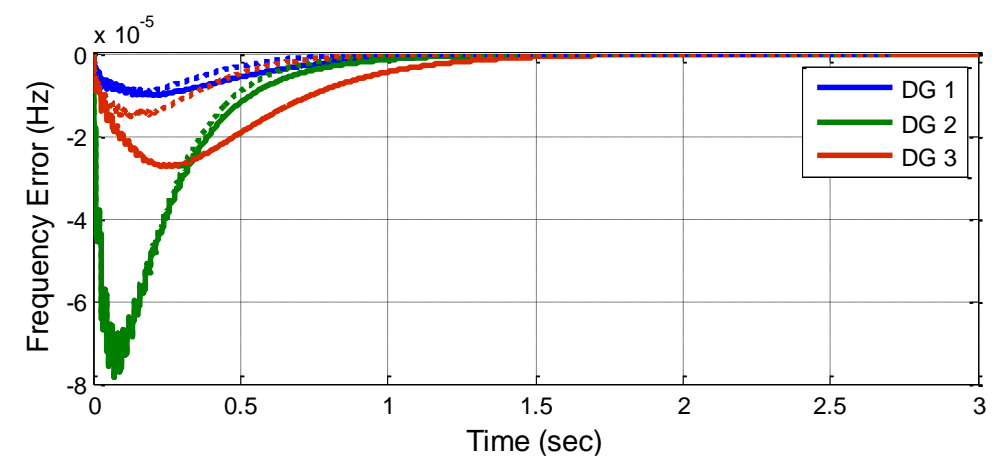

Figure 4.13: Frequency Error of VSI inverters in case 1 and 2. (Solid lines: case 1, Broken lines: Case 2)

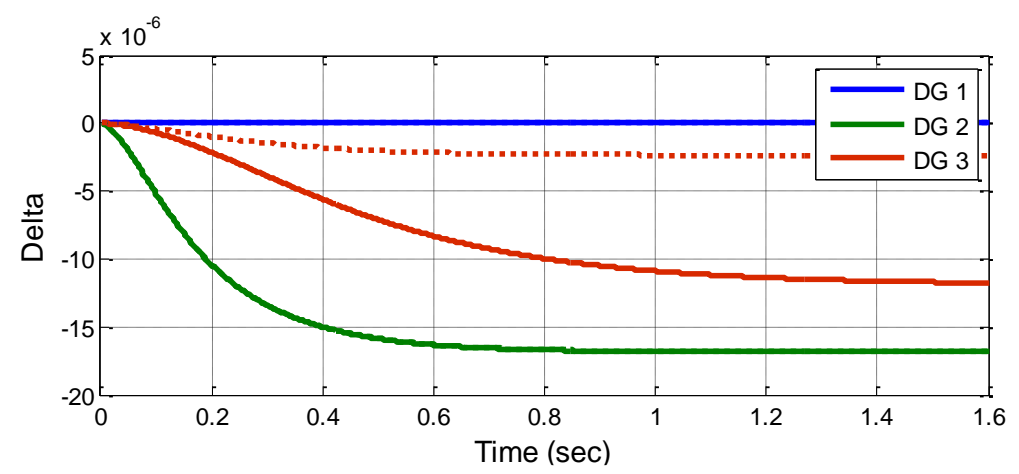

Figure 4.14: Change of delta in case 1 and 2. (Solid lines: case 1, Broken lines: Case 2) 


\section{3) Case 3}

In the third case, a PQ inverter is added to bus 1, producing power based on the set-point it receives through the communication link from VSI1. The participation factor for the PQ inverter here is chosen to be $25 \%$ of a total load change. Figure 4.15 shows the frequency error of a VSI inverter when the participation factor is 0 and $25 \%$. In fact, solid lines demonstrate the frequency error when the PQ inverter does not participate in load change and the broken line shows frequency error when the PQ inverter participation factor is equal to $25 \%$.

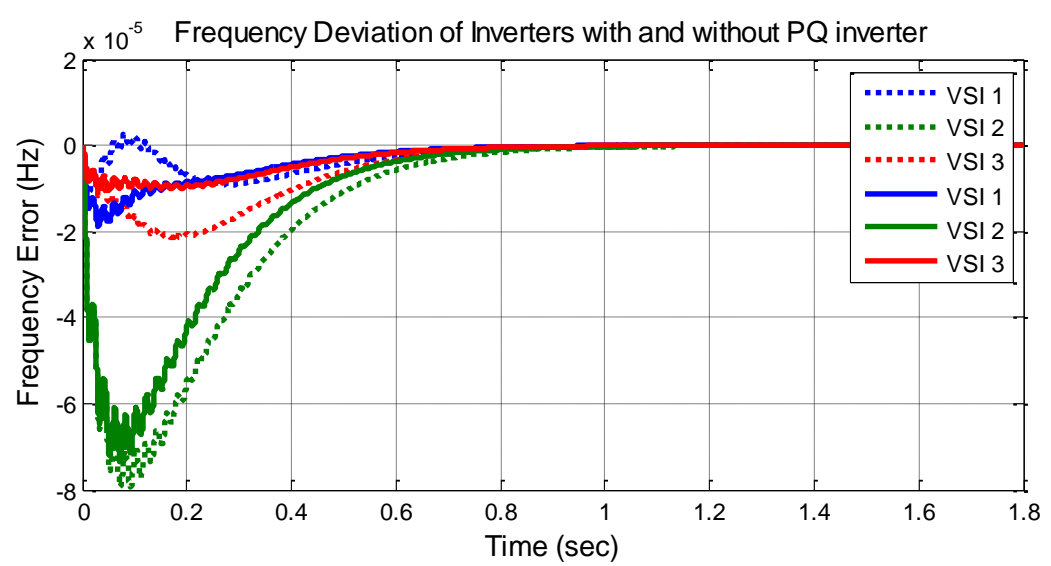

Figure 4.15: Frequency error of VSI inverters with and without PQ inverters participation.

Finally, figure 4.16 depicts the contribution of DGs in producing the demand power when the participation factor is between $0 \%$ and $25 \%$.

a) Without $P Q$ inverters

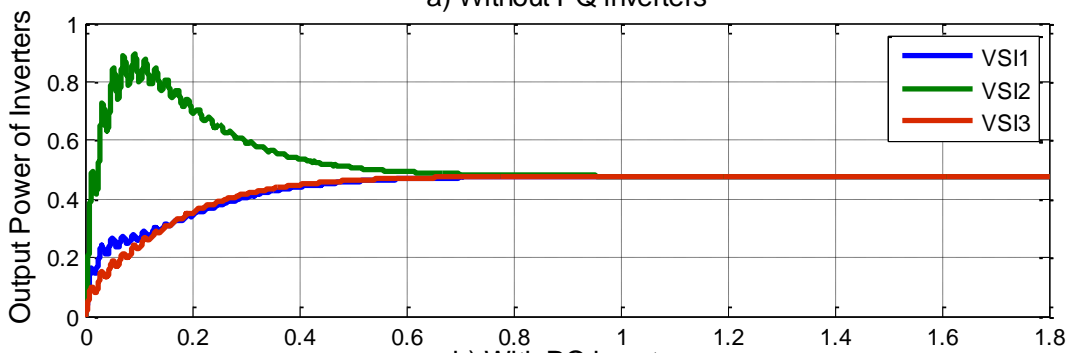

b) With $P Q$ inverter

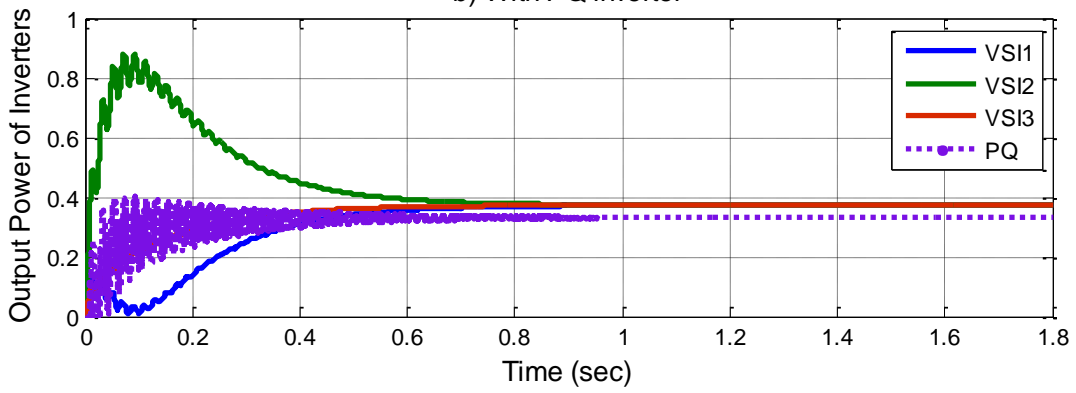

Figure 4.16: Output power of VSI and PQ inverters with and without PQ inverter Participation 


\subsection{PQ CONTROL SIGNAL (SET-POINT SIGNAL)}

The PQ inverters provide active and reactive power for the microgrid based on the set-point signal they receive. This signal can be achieved by communicating with one or more VSI inverters. In fact, they are supposed to provide a specific portion of the total change of demand based on their participation factor. Equation 4.1 shows the power balance in the microgrid, which means the total change in load active power would be equal to the summation of changes in the inverter's output power. Then, by substituting each inverter's output power, equation 4.2 will be achieved. In these equations, it is assumed that $n$ is the number of VSI inverters and $m$ is the number of PQ inverters.

$$
\begin{aligned}
& \Delta P_{\text {Load }}=\Delta P_{V S I 1}+\ldots+\Delta P_{V S I n}+\Delta P_{P Q 1}+\ldots+\Delta P_{P Q m} \\
& \Delta P_{P Q x}=\alpha_{x} \Delta P_{L o a d} \\
& \frac{\Delta P_{P Q x}}{\alpha_{x}}=\Delta P_{V S I 1}+\ldots+\Delta P_{V S I n}+\Delta P_{P Q 1}+\ldots+\Delta P_{P Q x}+\ldots+\Delta P_{P Q m} \\
& \Delta P_{P Q x}\left(\frac{1-\alpha_{x}}{\alpha_{x}}\right)=\Delta P_{V S I 1}+\ldots+\Delta P_{V S I n}+\Delta P_{P Q 1}+\ldots+\Delta P_{P Q(x-1)}+\Delta P_{P Q(x+1)} \ldots+\Delta P_{P Q m} \\
& \Delta P_{P Q x}=\left(\frac{\alpha_{x}}{1-\alpha_{x}}\right)\left(\sum \Delta P_{V S I i}+\sum_{j \neq x} \Delta P_{P Q j}\right)
\end{aligned}
$$

So, based on what we have in equation 4.5, it can be seen that a possible set-point signal for PQ inverters is formed by adding the output power changes of the other inverters. It is obvious that such a signal may need a lot of communication connecting a PQ inverter to all other nodes as it is shown in figure 4.17.

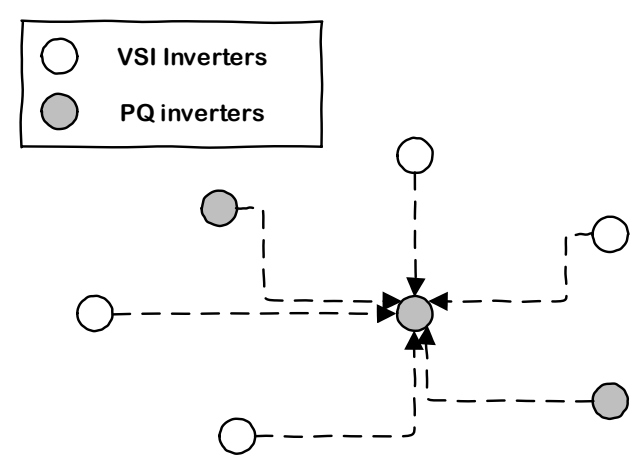

Figure 4.17: Required communication for CSI control in the first solution 
In order to come up with a better solution, the communication link between PQ inverters can be eliminated, which means PQ inverters will only use the data coming from the VSI inverters. Obviously, the advantage is reducing a portion of communication links but at the same time, each PQ node is required to know the participation factor of other PQ inverters.

It does not seem to be difficult as this factor can be considered constant during operation of microgrids. However, it should be noticed that if any new PQ inverter starts working inside the microgrid or any of existing PQ inverters stop working, the knowledge of other PQ inverters has to be updated for appropriate operation.

$$
\begin{aligned}
& \Delta P_{P Q i}=\alpha_{i} \Delta P_{\text {Load }} \\
& \Delta P_{P Q x}\left(\frac{1-\alpha_{x}}{\alpha_{x}}\right)=\Delta P_{V S I 1}+\ldots+\Delta P_{V S I n}+ \\
& \alpha_{1} \Delta P_{\text {Load }}+\ldots+\alpha_{x-1} \Delta P_{\text {Load }}+\alpha_{x+1} \Delta P_{\text {Load }}+\ldots+\alpha_{m} \Delta P_{\text {Load }} \\
& \Delta P_{P Q x}\left(\frac{1-\alpha_{x}}{\alpha_{x}}\right)=\Delta P_{V S I 1}+\ldots+\Delta P_{V S I n}+\left(\alpha_{1}+\ldots+\alpha_{x-1}+\alpha_{x+1}+\ldots+\alpha_{m}\right) \frac{\Delta P_{P Q x}}{\alpha_{x}} \\
& \Delta P_{P Q x}\left(\begin{array}{c}
1-\sum_{i=1}^{m} \alpha_{i} \\
\alpha_{x}
\end{array}\right)=\Delta P_{V S I 1}+\ldots+\Delta P_{V S I n} \\
& \Delta P_{P Q x}=\left(\begin{array}{c}
\alpha_{x} \\
1-\sum_{i=1}^{m} \alpha_{i}
\end{array}\right) \sum_{j=1}^{n} \Delta P_{V S I j}
\end{aligned}
$$

Equation 4.10 verifies that by having the signal from VSI inverters and knowing the participation factor of all PQ inverters, there is no more need to have the communication links between the PQ inverters. Figure 4.18 illustrates this solution. 


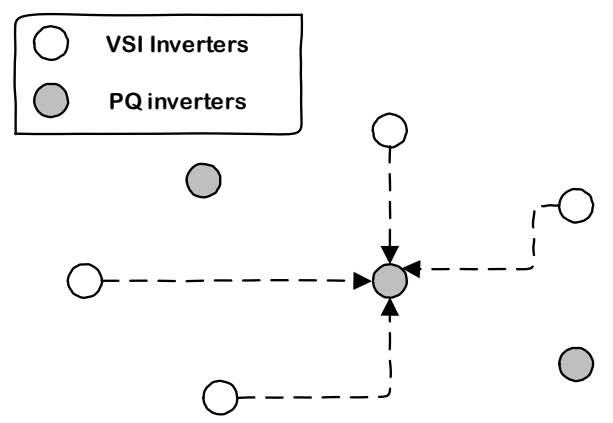

Figure 4.18: Required communication for CSI control in the second solution

However, this solution may be inapplicable in some situation where there are many VSI inverters possibly far apart from each other. So, in the following equation, the PQ signal is rebuilt in a way that it just needs to communicate with only one VSI inverter, which can be chosen based on how far it is. It should be noted that proper performance of PQ inverter and subsequently the whole system depends on only one signal, so it should be received by the PQ inverter without any delay or loss. However, for having a redundancy, it is possible to consider more than one (like two or three) communication link.

$$
\begin{aligned}
& \Delta \omega_{i}=\Delta \omega_{n i}-m_{P i} \Delta P_{V S I i} \\
& \text { AtSS: } m_{P 1} \Delta P_{V S I 1}=m_{P 2} \Delta P_{V S I 2}=\cdots=m_{P i} \Delta P_{V S I i} \\
& \Delta P_{P Q x}\left(\frac{1-\sum_{i=1}^{m} \alpha_{i}}{\alpha_{x}}\right)=\frac{m_{p i}}{m_{p 1}} \Delta P_{V S I i}+\frac{m_{p i}}{m_{p 2}} \Delta P_{V S I i}+\cdots+\Delta P_{V S I i}+\cdots+\frac{m_{p i}}{m_{p n}} \Delta P_{V S I i} \\
& \Delta P_{P Q x}\left(\frac{1-\sum_{i=1}^{m} \alpha_{i}}{\alpha_{x}}\right)=m_{p i} \Delta P_{V S I i}\left(\sum_{j=1}^{n} \frac{1}{m_{p j}}\right) \\
& \Delta P_{P Q x}=m_{p i} \alpha_{x}\left(\frac{\sum_{j=1}^{n} \frac{1}{m_{p j}}}{1-\sum_{i=1}^{m} \alpha_{i}}\right) \Delta P_{V S I i}
\end{aligned}
$$

Figure 4.19 shows the required communication link in the last solution. 


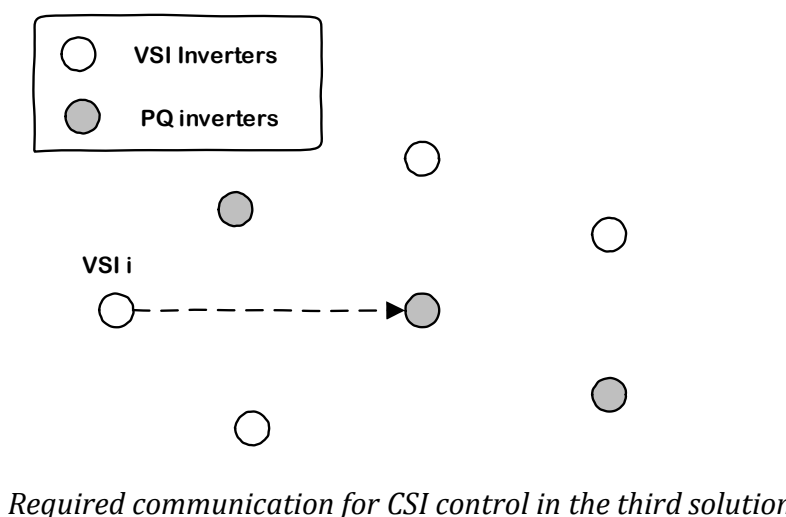

Figure 4.19: Required communication for CSI control in the third solution

\subsubsection{Simulation ReSUlT}

\section{1) First Scenario}

To continue this section, a PQ inverter is first added to the understudied microgrid at bus 1 (section 4.2.1). Then, the different possible signals are examined to see the performance of the system. In this system, there are 3 VSI inverters that can communicate with the PQ inverter. Four different cases are considered here. In the first case, only VSI 1 is communicating with the PQ inverter located at bus 1 . In the second case, VSI 2 has the appropriate link to signal to the PQ inverter. In the third case, VSI 3 is sending the signal, and finally, in the last case, all three VSIs are sending signals to the PQ inverter. Simulation results are depicted in the following. These results are based on a load change in bus 1 (load 1 is changing). Figure 4.20 shows the frequency deviation of all three VSI of the microgrid under four different cases.

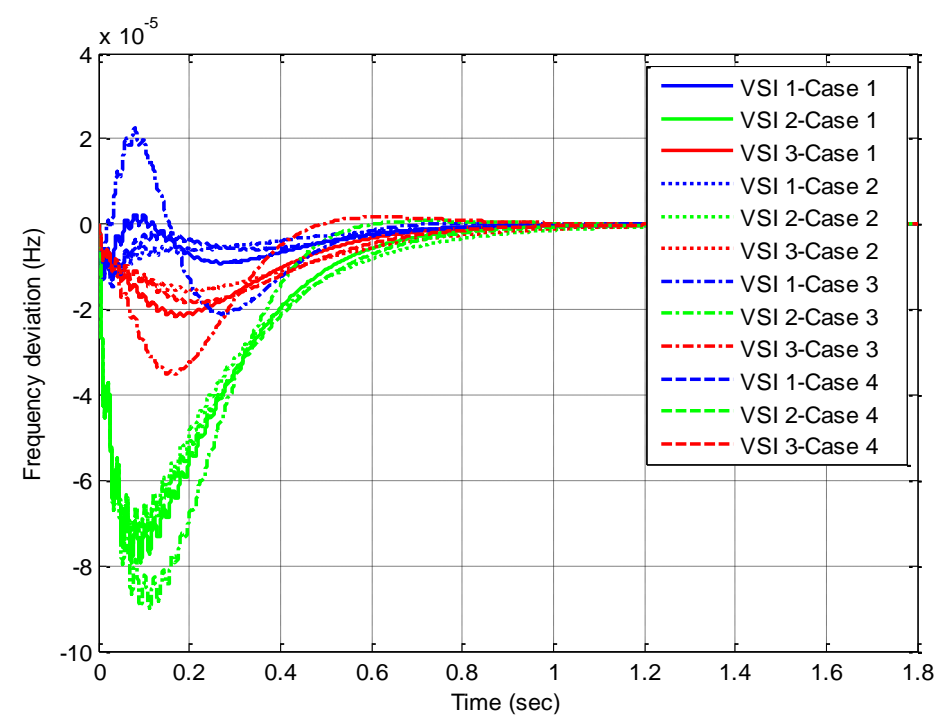

Figure 4.20: Reference frequency deviation of all VSI inverters of microgrid for four cases provided for signaling the PQ 
Then, in figures 4.21-4.23, the results related to frequency deviation is displayed in separate graphs. In these graphs, the blue line represents the frequency deviation related to the first case, in which only VSI 1 is communicating with the PQ. Then, the green line shows the results related to case 2 . The red line is related to case 3 , and finally, the grey line represents the $4^{\text {th }}$ case, in which the PQ is receiving signals from all three VSI inverters.

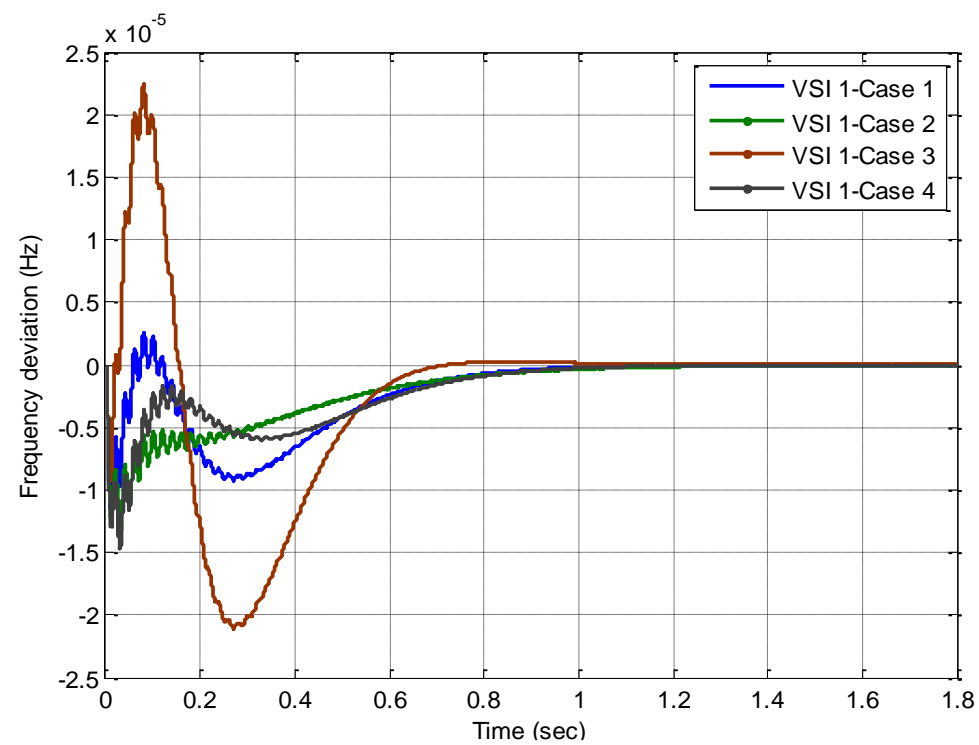

Figure 4.21: Reference frequency deviation of VSI 1 during all four cases provided for signaling the PQ inverter

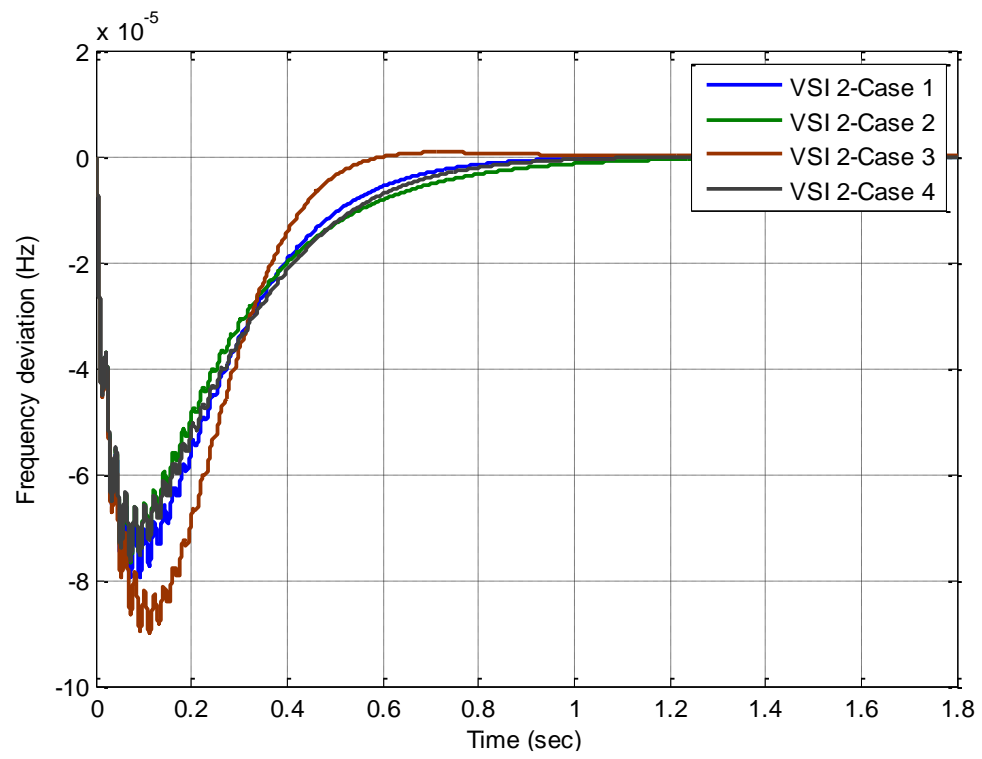

Figure 4.22: Reference frequency deviation of VSI 2 during all four cases provided for signaling the PQ inverter 


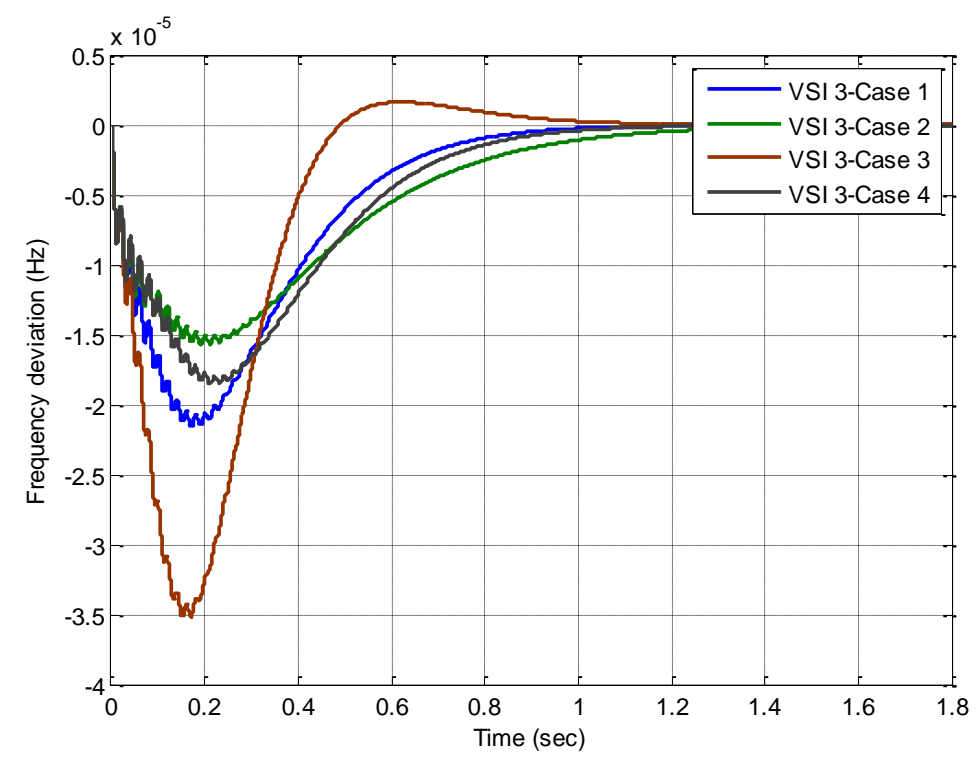

Figure 4.23: Reference frequency deviation of VSI 3 during all four cases provided for signaling the PQ inverter

Figure 4.24 shows the transient change of delta of VSI 2. The remaining figures 4.25-4.27 shows the output active power of each VSI inverter separately for all four cases.

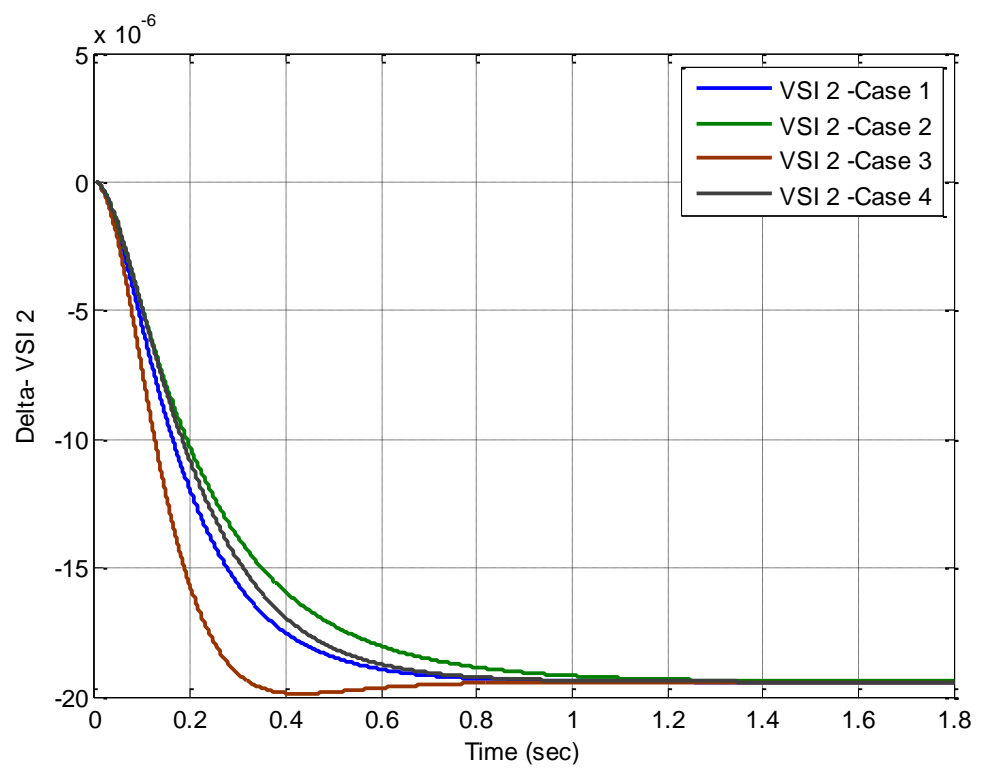

Figure 4.24: Change of delta related to VSI 2 of the microgrid for all four cases provided for signaling the PQ inverter 


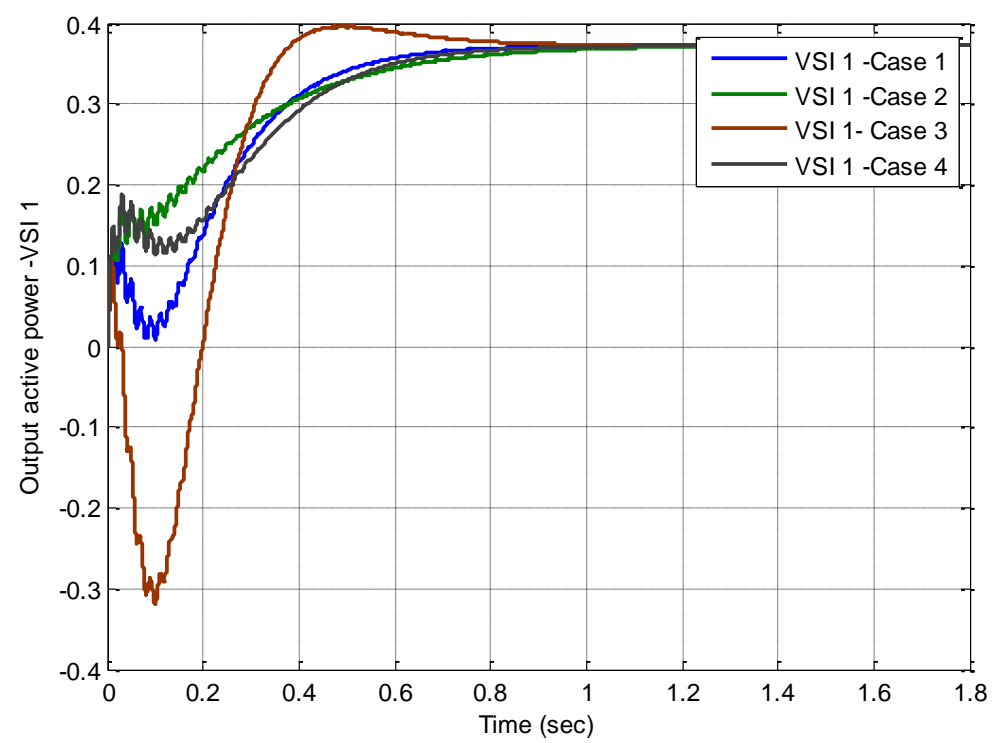

Figure 4.25: Change of output active power related to VSI 1 of the microgrid for all four cases provided for signaling the

$P Q$ inverter

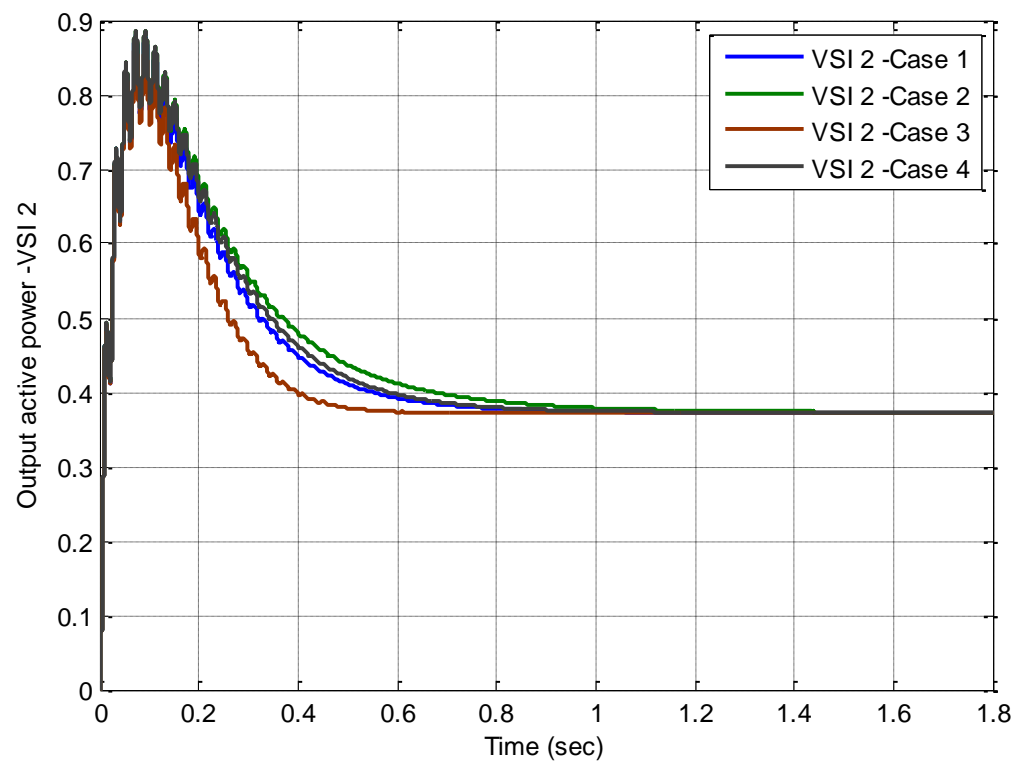

Figure 4.26: Change of output active power related to VSI 2 of the microgrid for all four cases provided for signaling the $P Q$ inverter 


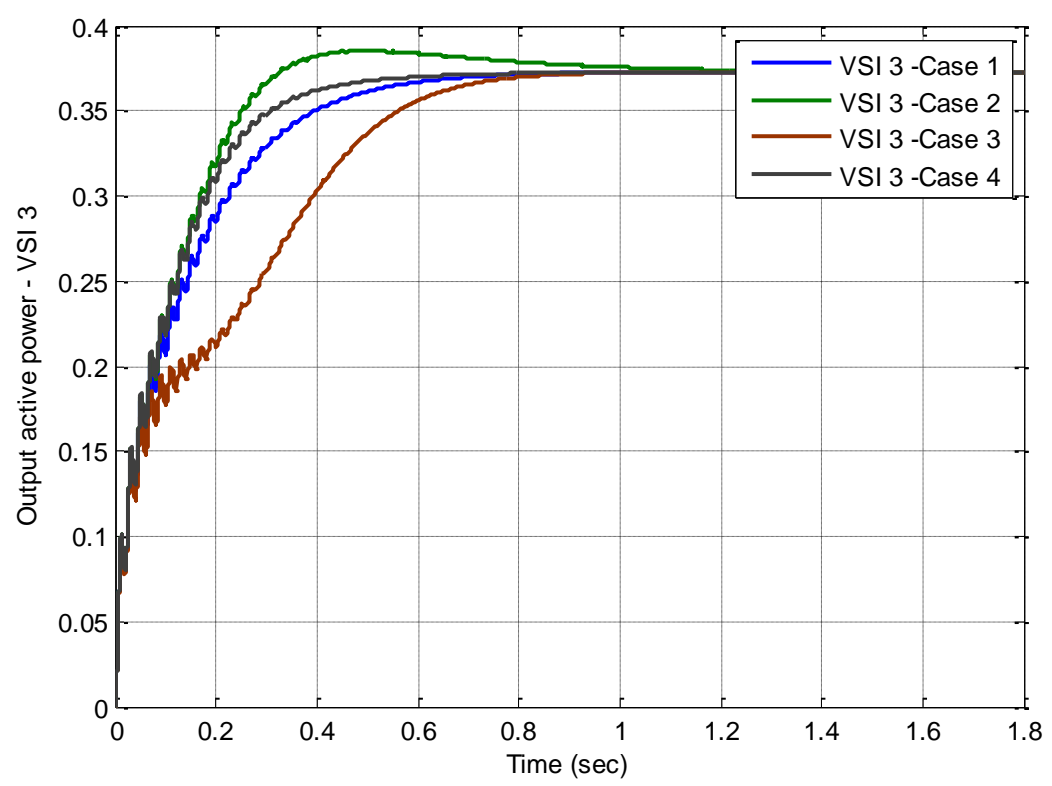

Figure 4.27: Change of output active power related to VSI 3 of the microgrid for all four cases provided for signaling the $P Q$ inverter

\section{2) Second Scenario}

In the previous scenario, the PQ inverter was located at bus 1 . In the second scenario, it has been relocated to bus 11. In order to compare the effect of choosing different communication links on the transient responses, the first and the second scenario has been repeated several times while considering the change of demand in different buses. An objective function is used to measure the transient quality of frequency response of the microgrid.

Equation 4.16 states the objective function used in these simulations. Figure 4.28 demonstrates the results on a bar graph. In graph a, the PQ inverter is located at bus 1 and in graph b, it is located at bus 11. Each graph shows the objective function value for load change in bus 1, 7, 4, 9 and 11 separately. The main conclusion gained from these graphs is that each signal selection can be good for specific scenarios and cases, but having some more signals means there will be an average so it can help to avoid the worst case scenarios.

$$
O B J=\int \Delta f^{2} d t
$$



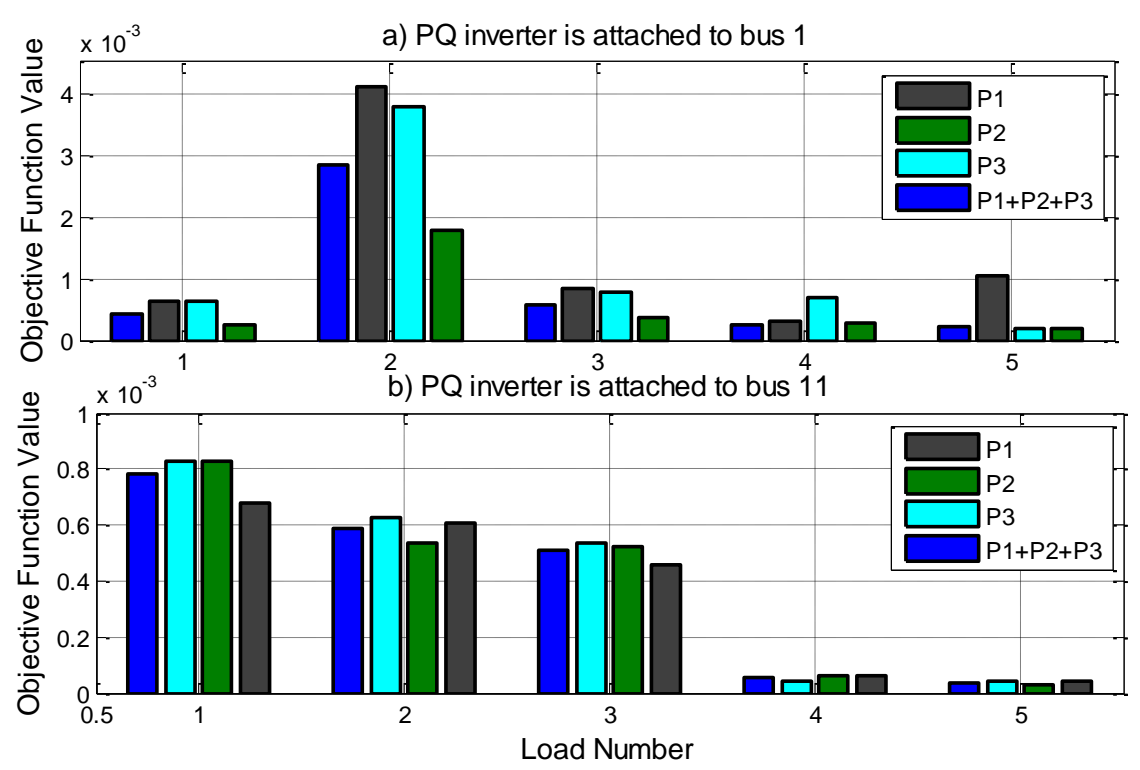

Figure 4.28: Bar graphs related to objective function values in different scenarios

\subsection{NOVEL LOAD FREQUENCY CONTROL OF MICROGRID WITH TIE LINE ERROR}

In this section, a novel load frequency control that can significantly improve the frequency response to load fluctuations is developed; the proposed approach is trying to divide the microgrid into virtual areas and control the frequency of the microgrid by using the tie line error, which is called Virtual Area Control Error (VACE), instead of just using the frequency deviation error.

In order to evaluate the proposed concept of frequency control, a detailed model of a microgrid implemented in PSCAD software is used. The microgrid has two generating units, a Photovoltaic (PV) source coupled with a Battery Energy Storage System (BESS), and a rotating synchronous generator, described in section 4.4.1. After investigating the proposed concept for frequency control on a detailed microgrid, a systematic way is introduced later in 4.5. which forms the state space model of microgrid when the system is divided into different zones and the tie line error is needed to be considered as new input matrix for further control purposes.

In the following sections, the configuration of the PV and battery system is briefly described. More details about the PSCAD modeling is provided in the appendix.

\subsubsection{MiCROGRID TEST SYSTEM}

A schematic of the microgrid under study is shown below. It has two generating units, a Photovoltaic (PV) source coupled with a Battery Energy Storage System (BESS) and a rotating synchronous generator as schematically shown in Figure 4.29. 


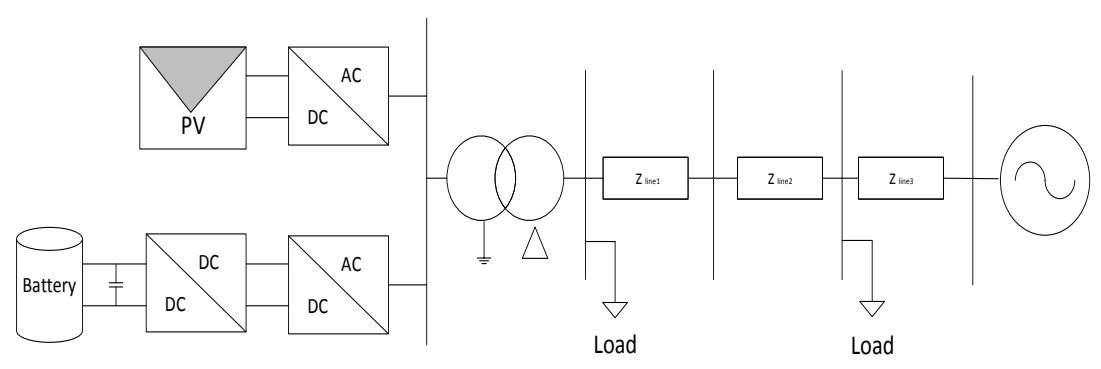

Figure 4.29: Schematic of the "Test" Microgrid

The three phase microgrid block depicted in Figure 4.30 is comprised of a Phase-Locked Loop (PLL) that ensures the output currents are in phase with the generator's terminal voltages. The PV panel/battery system is connected as shown in Figure 4.29.

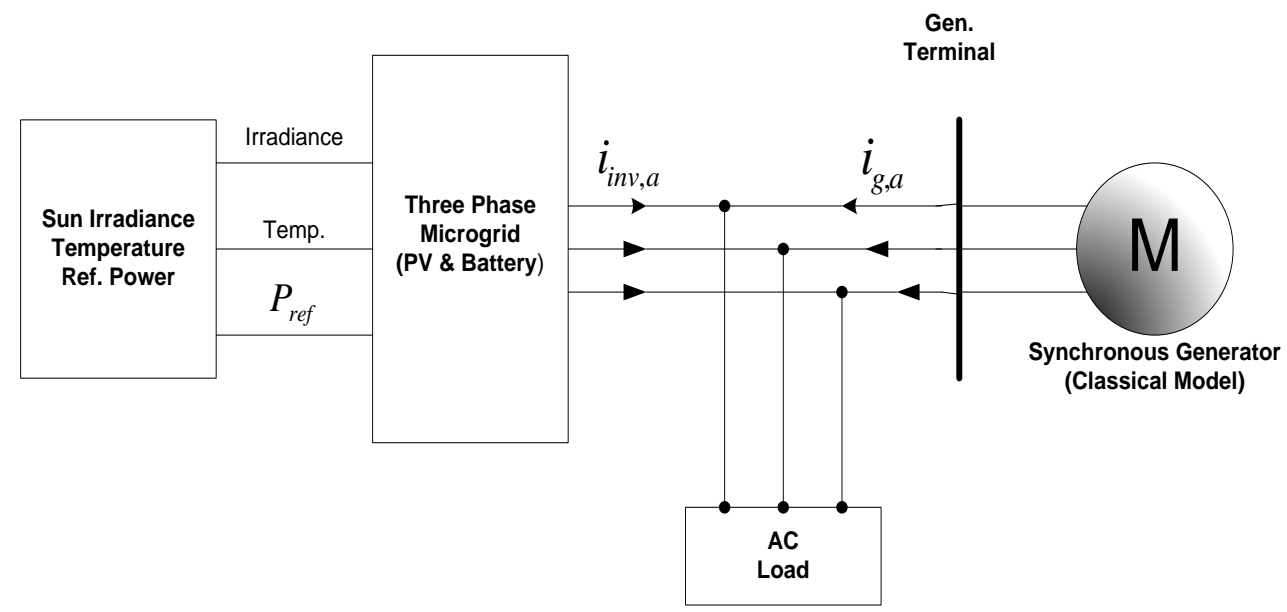

Figure 4.30: Schematic Block Diagram of a Three Phase Microgrid System

The three phase microgrid contains a hybrid source consisting of a Photovoltaic Energy Conversion System (PVECS), a BESS and their converters.

The objective of this section is to utilize the PV/Battery system for implementing a novel frequency control of the microgrid. The hybrid system is to generate a pre-defined or reference value of active power. This value can either be delivered to the grid or it can be used to supply a native load.

When there is excess in power generation from the sun with respect to the pre-defined value, the excess power is used to charge the battery. However, if the power generated by the PV is less than the pre-defined value, the BESS will discharge (provided as charged) to cover the deficiency.

\section{Modeling the Detailed Microgrid in PSCAD® Software}

PSCAD® stands for Power System Computer Aided Design. This software is used to model and analyze the PV inverter system in this research. The simulation environment includes a default master library that 
includes typical circuit components of a Spice library but goes one step further in complexity by also including items ranging from advanced power electronic devices all the way to rotating machines' models such as a synchronous generator. The strength and uniqueness of the program is the ability to integrate complex power electronic circuits with large power systems and view the system responses in the time and frequency domains.

\subsubsection{NOVEL LOAD FREQUENCY CONTROL DESIGN}

As previously mentioned, there are different approaches for controlling the frequency of microgrids; almost all of them consider the low voltage microgrid as one area and most of them use the frequency error as ACE. One of the main contributions of this study is introducing a new approach for load frequency control in which each fast response generating sources (like PV/Batt system) takes the responsibility of a virtual area around them and tries to support them in a transient period whenever there is a load change in their own area. It should be noted that the assigned ACE to each area may differ from other areas due to the specific characteristics of that area's power source. In this part of the research, a specific ACE is defined for PV/Batt system that can improve the load frequency control compared to the usual approaches.

Considering the configuration of the system used in this chapter, we will divide the microgrid into two areas. Figure. 4.31 shows the way we made up the areas in this system.

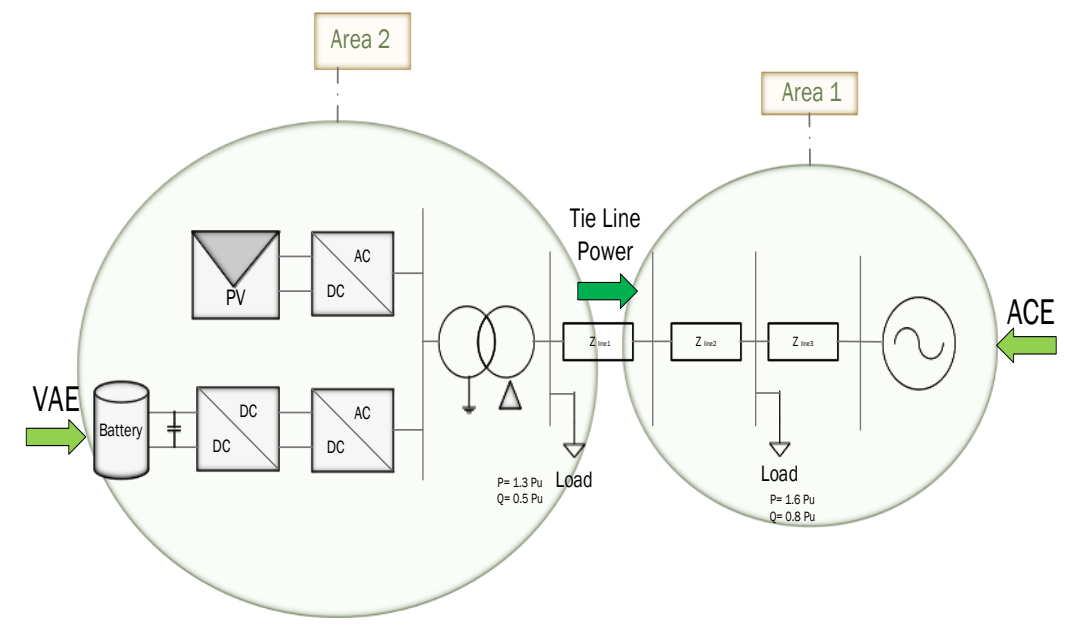

Figure 4.31: Dividing the System under Study into Two Areas

In fact, in this case, we have two assigned areas and each of them is supposed to take care of its own frequency, so whenever the load is changed in each area, the particular area does its best to control the frequency. In this case, there is an imaginary tie line trying to have a predetermined value for its power flow. The error of tie-line power from the predetermined value is added as ACE to the set-point of PV/Batt 
system. So, the important term defining our new ACE is a coefficient of tie-line error, but this term alone cannot work for a long period due to the finite amount of power the battery is producing. As a result, we need another term that can bring the characteristic of the battery into the defined ACE. So, the ACE should be multiplied by the battery's state of charge subtracted by the minimum SOC.

This new measurement to assess performance is called Virtual Area Control Error (VACE) and is introduced as equation 4.17. Figure. 4.32 also demonstrates the block diagram of VACE.

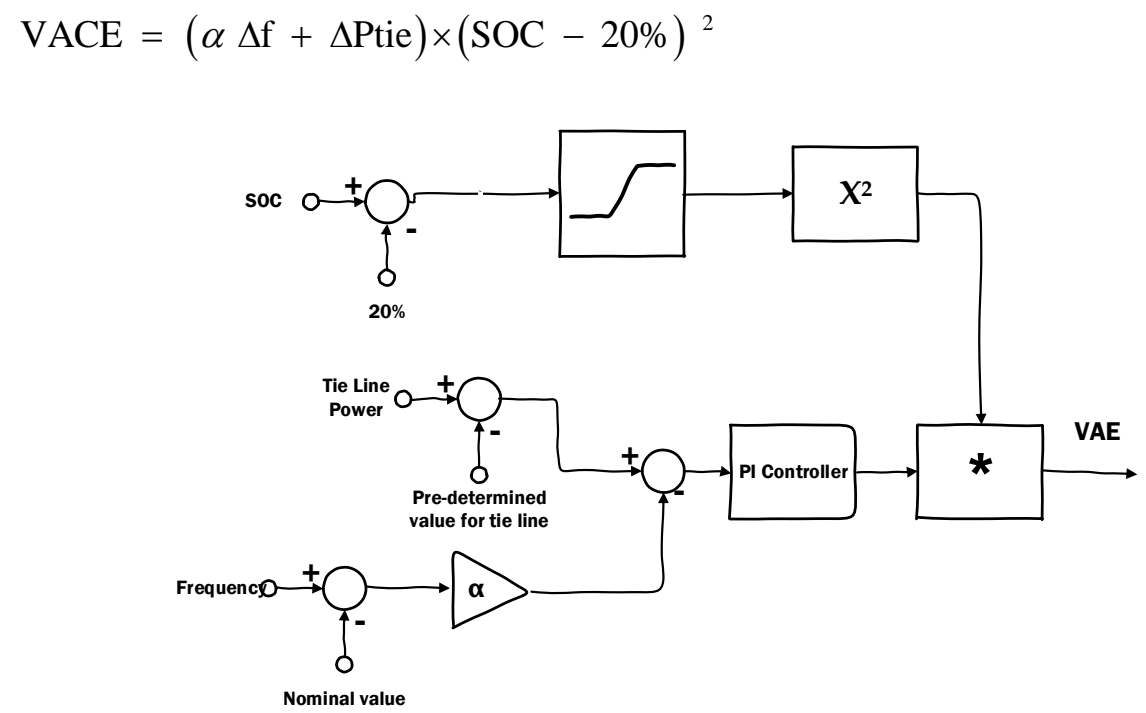

Figure 4.32: Virtual Area Control Error (VACE) Diagram

The term of SOC-20\% helps area two (PV/Batt) to consider its own situation in addition to the frequency's condition. The effectiveness of this approach is shown in the simulation section.

\subsubsection{Simulation ReSUlt}

In order to investigate the application of the strategy above, it has been tested on the previously mentioned detailed model of a microgrid in a PSCAD environment which was described at the beginning of this section.

Three different scenarios have been tested and the results were then compared together.

\section{$>$ First scenario}

In the first scenario the PV/Battery is assumed to produce a 1.5 pu power which is constant during the simulation. It is working as a PQ inverter. In this case the synchronous generator, which is a rotating machine with inertia, is responsible for the microgrid load frequency control. The schematic of load frequency control in this scenario is depicted in Figure 4.33. 


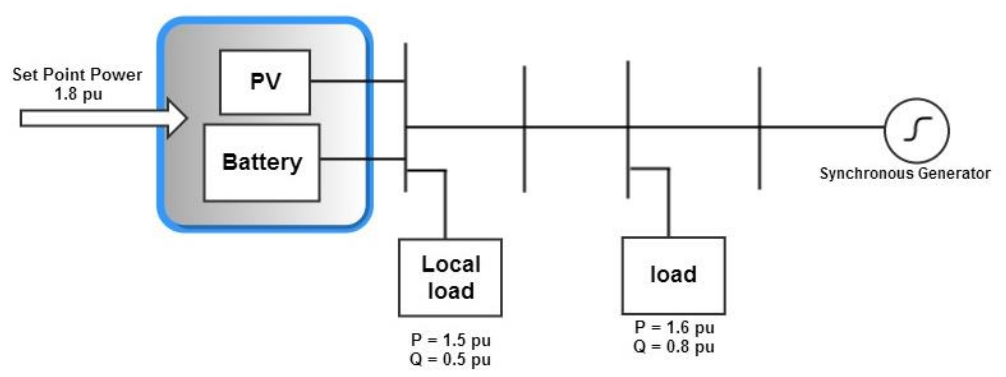

Figure 4.33: Load Frequency Control in First Scenario

\section{Second scenario}

In the second scenario, the common ACE of the microgrid, which is the frequency error compared to the reference value, is also sent to the PV/Battery system so the PV/Battery helps the synchronous machine to bring back the frequency to the nominal value. The simulation results shown afterward demonstrate that there is an improvement in the frequency deviation compared to the first scenario. Yet a better result is achieved through the proposed approach discussed in this research as the third scenario. The second and third cases are compared with the first one to show the improvement in frequency control. Figure 4.34 shows the load frequency control schematic of the second scenario.

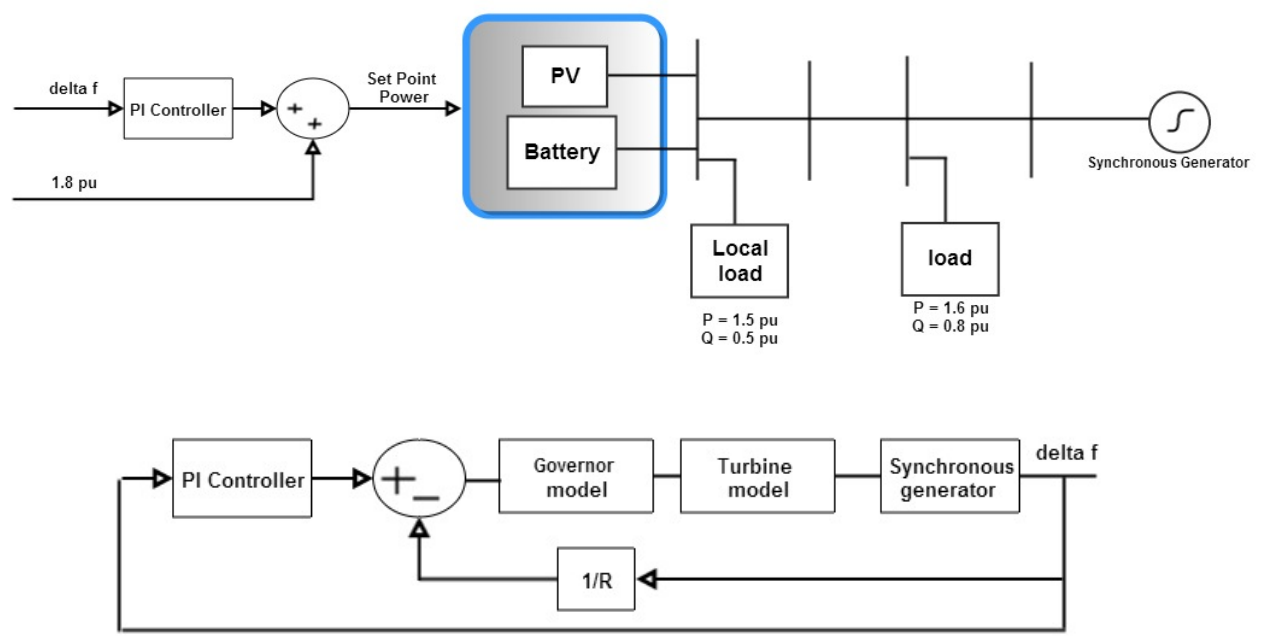

Figure 4.34: Load Frequency Control in Second Scenario

\section{$>$ Third scenario}

In the third case, the ACE used to adjust the PV/Batt set-point power, is changed from frequency deviation to VACE (which is the tie line error of virtual area considering the state of charge of battery). In fact, in 
this case, we have two assigned areas where each of them is supposed to take care of its own frequency, so whenever the load is changed in each area, the particular area does its best to control the frequency. In this case, there is an imaginary tie line trying to have a predetermined value for its power flow. The error of tieline power from the predetermined value is added as ACE to the set-point of PV/Batt system. Finally, the situation of the battery will be kept in mind by multiplying a term including battery's SOC to the ACE. The diagram of the third scenario's frequency control is shown in Figure 4.35.

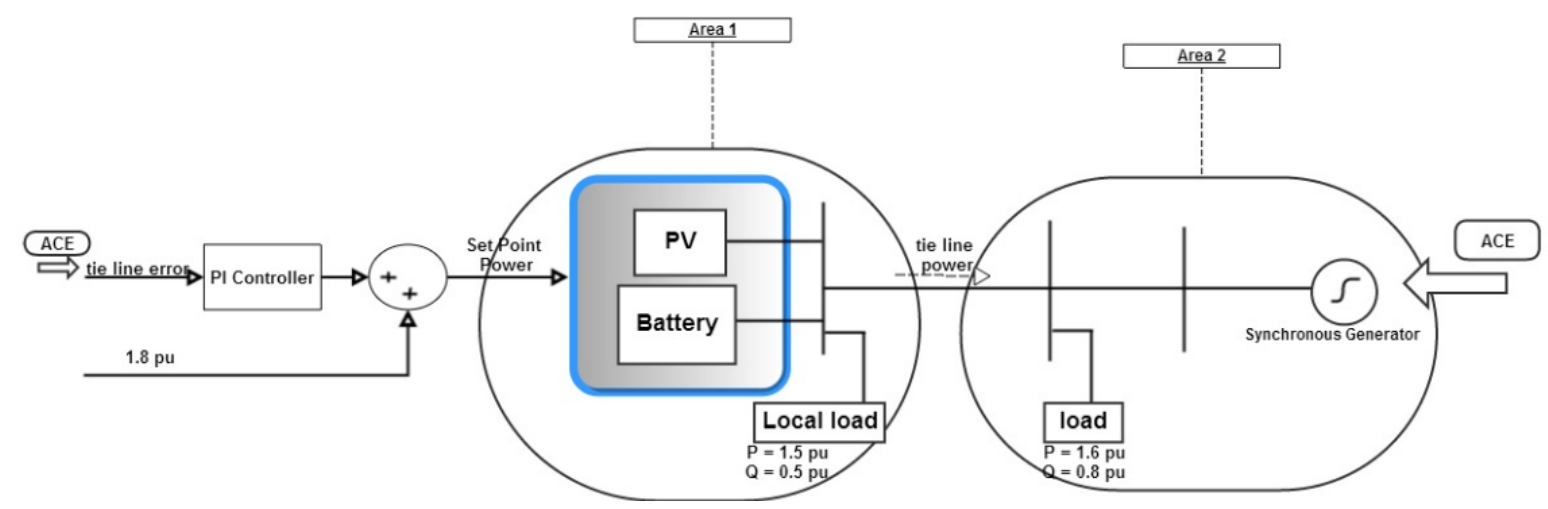

Figure 4.35: Load Frequency Control in Third Scenario

The results show that this approach can cause a significant improvement in controlling the frequency of microgrids. In other words, to have better frequency control, each fast response inverter based micro source is supposed to be responsible for a group of loads near its location, forming an area connected to a microgrid via an imaginary tie line. So, if any load in their dominant area changes, the micro sources can change their set-point to keep the tie-line power flow constant equal to its pre-determined value. However, characteristics of the micro source can make some changes in final VACE given to the DG.

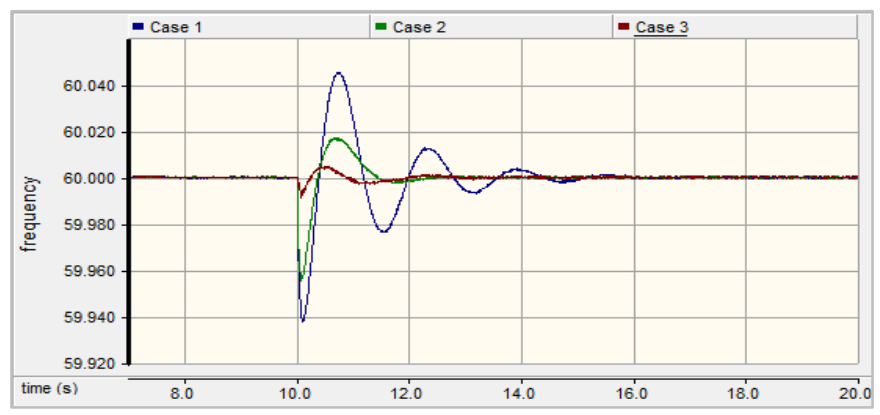

Figure 4.36: Comparing Frequency Fluctuation in Three Cases. 


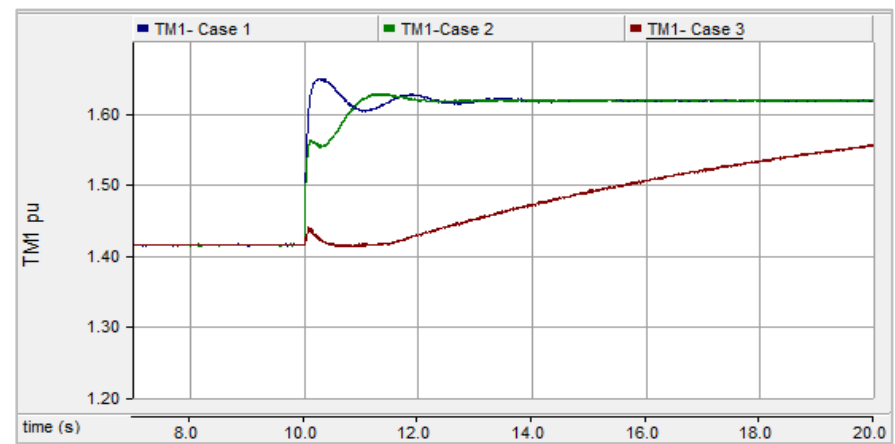

Figure 4.37: Comparing Torque of Synchronous Machine in Three Cases.

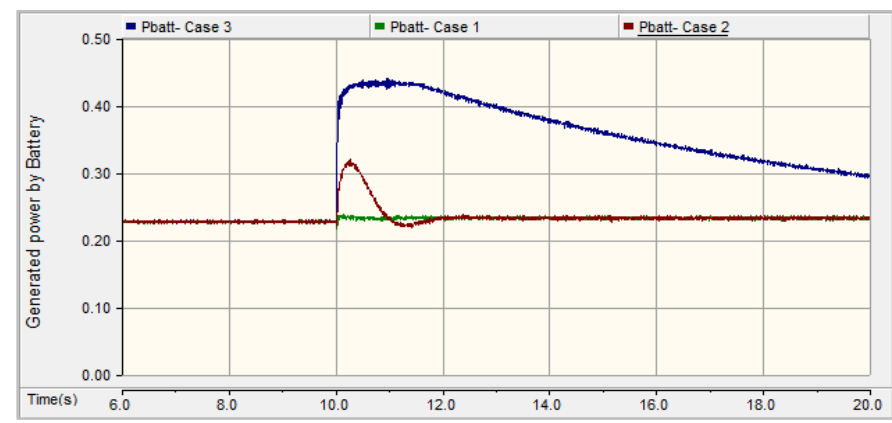

Figure 4.38: Power Generated by Battery in Three Cases.

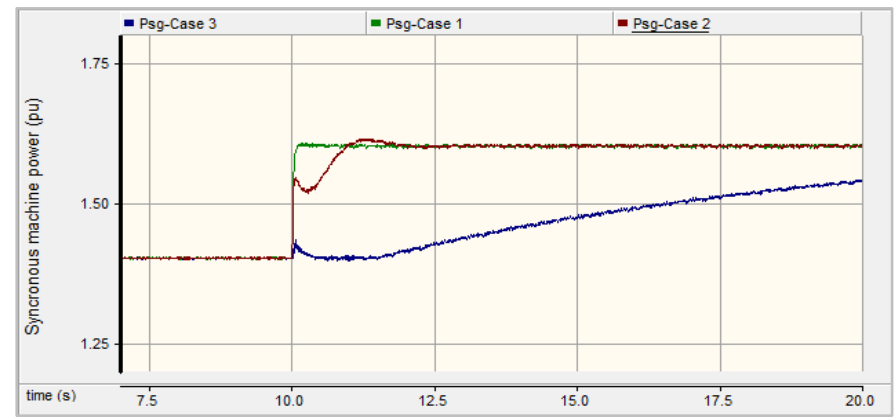

Figure 4.39: Power Generated by Synchronous Machine in Three Cases.

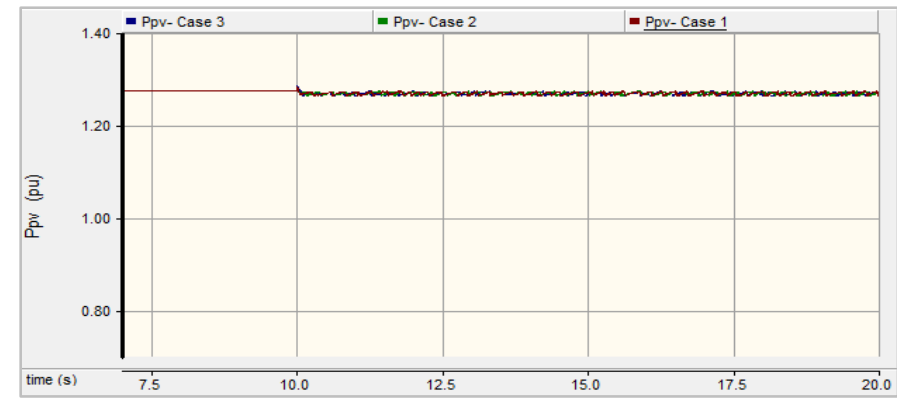

Figure 4.40: Generated Power by PV in Three Cases. 


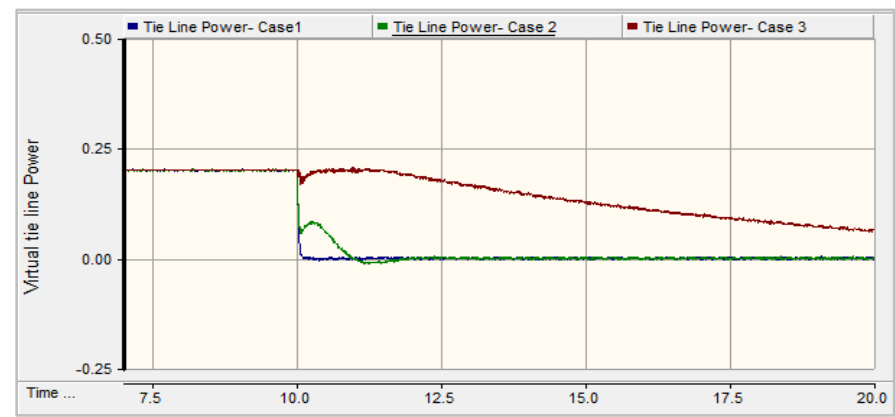

Figure 4.41: Virtual Tie-Line Power in Three Cases.

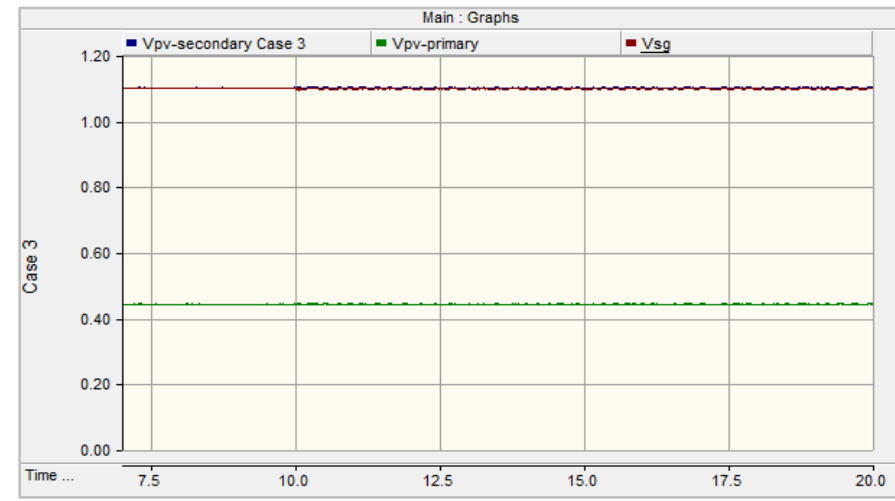

Figure 4.42: Output Voltage of Synchronous Machine and PV

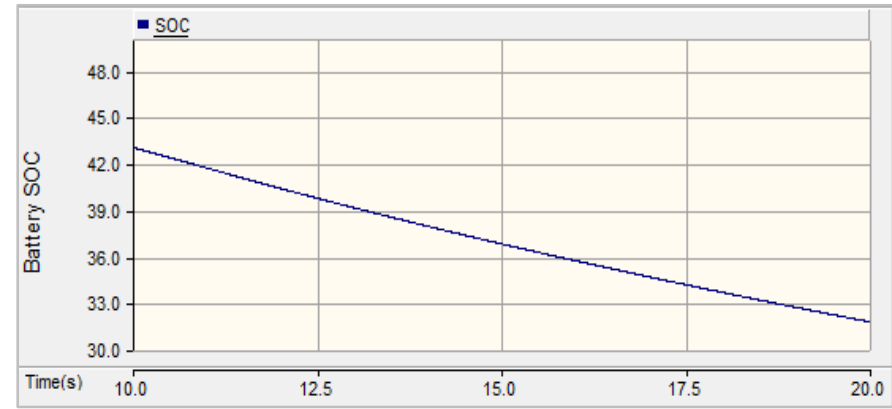

Figure 4.43: SOC of Battery in Third Case.

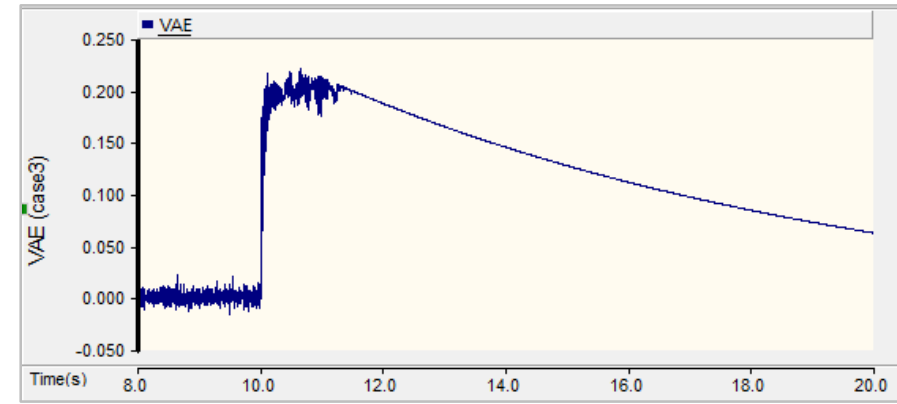

Figure 4.44: Virtual Area Error (In Third Case) 
Figures $4.36-4.44$ show the result of the simulation. In figure 4.36 , it can be seen that the frequency in case three is much better than the other two cases.

\subsection{State Space Formation of Microgrids Considering Virtual Tie Lines}

In this section, the state space model of a microgrid when considering a virtual tie line, is formed to design different controllers for the micro sources inside the microgrid. At first, the PI controller is designed and after that, robust $\mathrm{H} \infty$ controller is proposed to solve the frequency regulation problem.

\subsubsection{PI CONTROLLER}

An isolated microgrid with three VSI inverters is used to demonstrate the validity of the secondary frequency control. Figure 4.45 depicts the single line diagram of the understudied microgrid. The nominal voltage is $380 \mathrm{~V}$ and the nominal frequency is $60 \mathrm{~Hz}$. This microgrid is divided into two areas, trying to regulate the power going through the tie line.

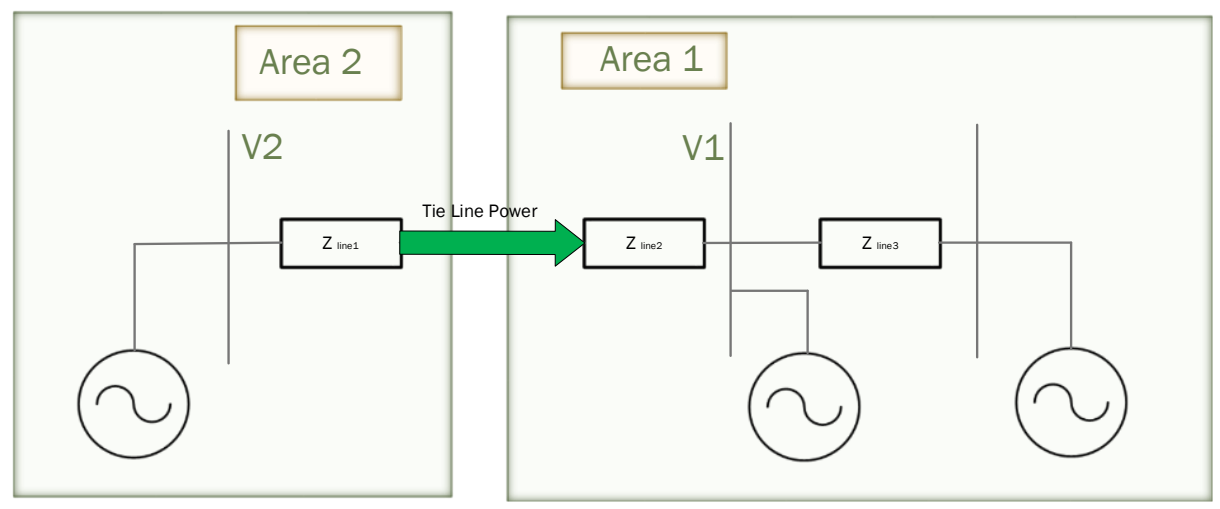

Figure 4.45: single line diagram of microgrid with tie line

After coming up with the system's steady space according to the modeling approach (provided in the previous chapter), it is important to find out the tie-line power flow equation based on the state space variable in order to use it for frequency control and tie-line power regulation. The equations below form the tie-line power from scratch and finally rebuilt it in equation 4.26 based on a constant matrix multiplied by the state space variable.

$$
\begin{aligned}
& P_{\text {tieline }}=V_{2} \times I_{\text {tieline }} \\
& \Delta P_{\text {tieline }}=\left(V_{2}+\Delta v_{2}\right) \times\left(I_{\text {tieline }}+\Delta i_{\text {tieline }}\right)-V_{2} \times I_{\text {tieline }} \\
& \Delta P_{\text {tieline }}=I_{\text {tieline }} \Delta v_{2}+V_{2} \Delta i_{\text {tieline }}+\Delta v_{2} \Delta i_{\text {tieline }}
\end{aligned}
$$




$$
\begin{aligned}
& \Delta P_{\text {tieline }}=I_{\text {tieline }-d} \Delta v_{2 d}+V_{2 d} \Delta i_{\text {tieline-d }}+I_{\text {tieline }-q} \Delta v_{2 q}+V_{2 q} \Delta i_{\text {tieline }-q} \\
& \left\{\begin{array}{l}
\Delta i_{\text {tieline-d }}=A_{2} \Delta x \\
\Delta v_{2 d}=C C_{1} \Delta x \\
\Delta i_{\text {tieline-q }}=A_{3} \Delta x \\
\Delta v_{2 q}=C C_{2} \Delta x
\end{array}\right. \\
& \Delta P_{\text {tieline }}=\left(I_{\text {tieline-d }} C C_{1}+V_{2 d} A_{2}+I_{\text {tieline- } q} C C_{2}+V_{2 q} A_{3}\right) \Delta x
\end{aligned}
$$

After finding the tie line power equation, it can be used to compound the control signal feeding the controller. The PI controller will produce the $\Delta \omega_{n i}$ with respect to its input signal, which has the $\Delta \omega_{n}$ and $\Delta P_{\text {tie }}$ as consistent terms. The PI controller and its input and output signals are depicted in figure 4.46.

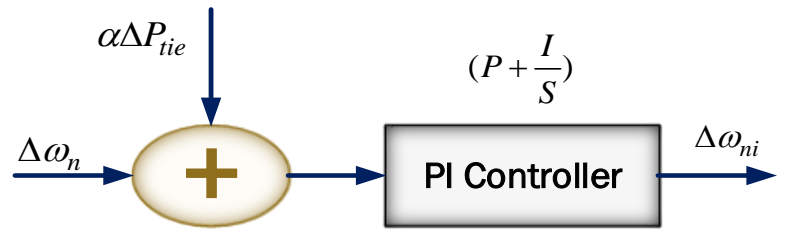

Figure 4.46: PI controller along with its input and output signals

Each VSI inverter of the microgrid here needs a secondary control signal as an input $\left(\Delta \omega_{n 1}, \Delta \omega_{n 2}\right.$ and $\Delta \omega_{n 3}$ ). Equation 4.27 shows how this input signal is built for the VSI 1. By substituting the $\Delta \omega_{n}$ and $\Delta P_{\text {tie }}$ from equation 4.26 , equation 4.27 can be rearranged so that it can form a new state equation shown in 4.35 . The procedure of coming up with this new state equation is shown through equations $4.28-4.34$. This process should be repeated for all three VSIs so that their state equations with their own control inputs are obtained.

$$
\begin{aligned}
& \Delta \omega_{n 1}=\left(\Delta \omega_{n}+\alpha \Delta P_{\text {tie }}\right)\left(P+\frac{I}{S}\right) \\
& \Delta \omega_{n 1}=\left(\Delta \omega_{n 1}-m_{p} A_{1} \Delta x+\alpha \Delta P_{\text {tie }}\right)\left(P+\frac{I}{S}\right) \\
& \Delta \omega_{n 1}\left(1-P-\frac{I}{S}\right)=-P m_{p} A_{1} \Delta x-\frac{I m_{p} A_{1} \Delta x}{S}+P \alpha \Delta P_{\text {tie }}+\frac{I \alpha \Delta P_{\text {tie }}}{S}
\end{aligned}
$$




$$
\begin{aligned}
& \Delta \omega_{n 1}\left(1-P-\frac{I}{S}\right)=P\left(-m_{p} A_{1} \Delta x+\alpha\left(I_{\text {tieline }-d} C C_{1}+V_{2 d} A_{2}+I_{\text {tieline }-q} C C_{2}+V_{2 q} A_{3}\right) \Delta x\right) \\
& +\frac{I}{S}\left(-m_{p} A_{1} \Delta x+\alpha\left(I_{\text {tieline-d }} C C_{1}+V_{2 d} A_{2}+I_{\text {tieline }-q} C C_{2}+V_{2 q} A_{3}\right) \Delta x\right) \\
& \Delta \omega_{n 1}\left(1-P-\frac{I}{S}\right)=P\left(-m_{p} A_{1}+\alpha I_{\text {tieline-d }} C C_{1}+\alpha V_{2 d} A_{2}+\alpha I_{\text {tieline }-q} C C_{2}+\alpha V_{2 q} A_{3}\right) \Delta x \\
& +\frac{I}{S}\left(-m_{p} A_{1}+\alpha I_{\text {tieline }-d} C C_{1}+\alpha V_{2 d} A_{2}+\alpha I_{\text {tieline }-q} C C_{2}+\alpha V_{2 q} A_{3}\right) \Delta x \\
& \left\{-m_{p} A_{1}+\alpha I_{\text {tieline-d }} C C_{1}+\alpha V_{2 d} A_{2}+\alpha I_{\text {tieline-q }} C C_{2}+\alpha V_{2 q} A_{3} \triangleq \psi\right. \\
& \Delta \omega_{n 1}((1-P) S)=I \Delta \omega_{n 1}+P \psi S \Delta x+I \psi \Delta x \\
& \Delta \dot{\omega}_{n 1}=\frac{I}{1-P} \Delta \omega_{n 1}+\frac{P}{1-P} \psi \Delta \dot{x}+\frac{I}{1-P} \psi \Delta x \\
& \Delta \dot{\omega}_{n 1}=\frac{I}{1-P} \Delta \omega_{n 1}+\frac{P}{1-P} \psi\left(A_{m g} \Delta x+B_{m g} u\right)+\frac{I}{1-P} \psi \Delta x
\end{aligned}
$$

The microgrid (the plant) state space matrices- $\mathrm{A}_{\mathrm{mg}}, \mathrm{B}_{\mathrm{mg}}, \mathrm{C}_{\mathrm{mg}}$ and $\mathrm{D}_{\mathrm{mg}}$ - will be combined with controller matrices, which are obtained from the three new state equations built through the procedure above. Equations 4.36 - 4.37 show the state space model of the plant and equations 4.38 - 4.39 shows those related to the controller. Also, it can be seen through equation 4.40 that the state variables of the controller are $\Delta \omega_{n 1}, \Delta \omega_{n 2}$ and $\Delta \omega_{n 3}$. Figure 4.47 shows these matrices and their dimensions in the state space block diagram of the whole system.

$$
\begin{gathered}
\text { Microgrid } \rightarrow\left\{\begin{array}{c}
\Delta \dot{x}=A_{m g} \Delta x+B_{m g} u \\
y=\Delta x
\end{array}\right. \\
\text { Feedback } \rightarrow\left\{\begin{array}{c}
\Delta \dot{\zeta}=A_{f} \Delta \varsigma+B_{f} y \\
u=\Delta \varsigma
\end{array}\right. \\
\Delta \varsigma=\left[\begin{array}{c}
\Delta \omega_{n 1} \\
\Delta \omega_{n 2} \\
\Delta \omega_{n 3}
\end{array}\right]
\end{gathered}
$$




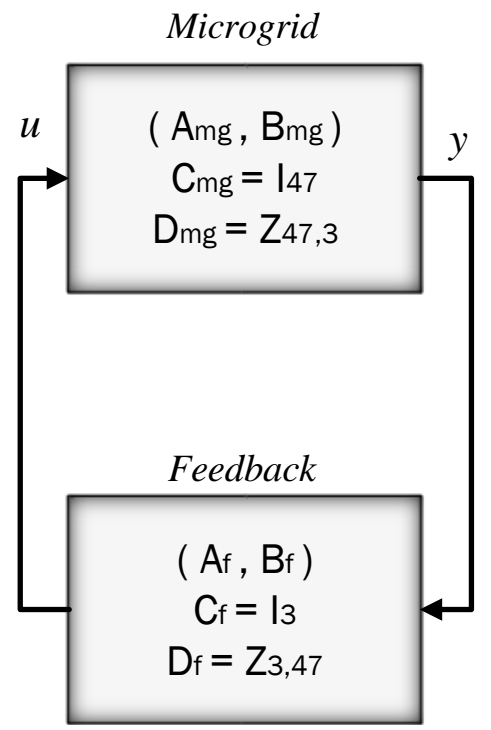

Figure 4.47: State space block diagram of the system

$\Delta \omega_{\mathrm{n} 1}$ is for the generating unit of area 1 and will be calculated as shown previously. However, $\Delta \omega_{\mathrm{n} 2}$ and $\Delta \omega_{\mathrm{n} 3}$ are related to the generating units of area 2 , so the only difference between their equations is the minus sign of tie-line power. Equation 4.41 shows the state space model of the augmented system. Figure 4.48 shows the output power of each generating units in presence and absence of the tie-line power error.

$$
\left[\begin{array}{c}
\Delta \dot{x} \\
\Delta \dot{\zeta}
\end{array}\right]=\left[\begin{array}{cc}
A_{m g} & B_{m g} \\
B_{f} & A_{f}
\end{array}\right]\left[\begin{array}{l}
\Delta x \\
\Delta \varsigma
\end{array}\right]
$$




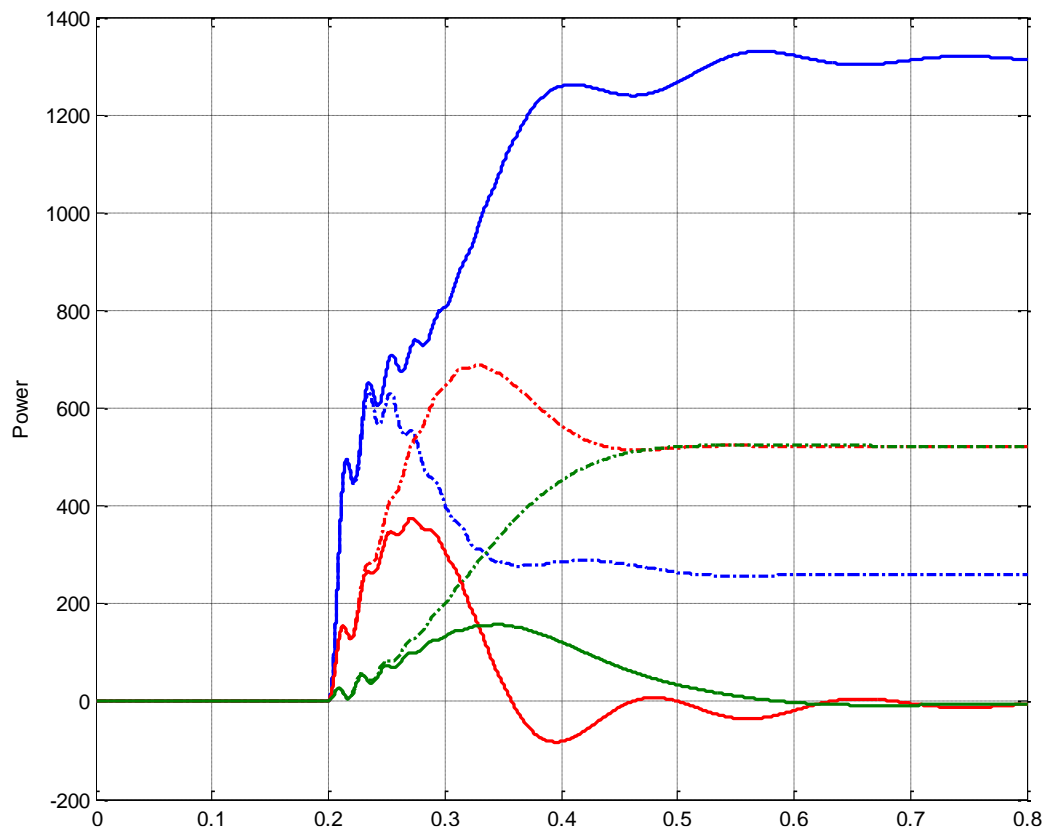

Figure 4.48: Output power of generating unit with and without tie line error (Solid lines: with tie line error - Broken line: without tie line error)

As seen in figure 4.48, when the tie line error is used as a term in the input control signal, the VSI 2 and 3 only interfere in the transient period, not the steady state, which means they will finally have a $0 \%$ contribution in power demand of area 1. It also shows that when the tie-line power is not used, all three VSI contribute in the load change based on their participation factors related to their ratings. 


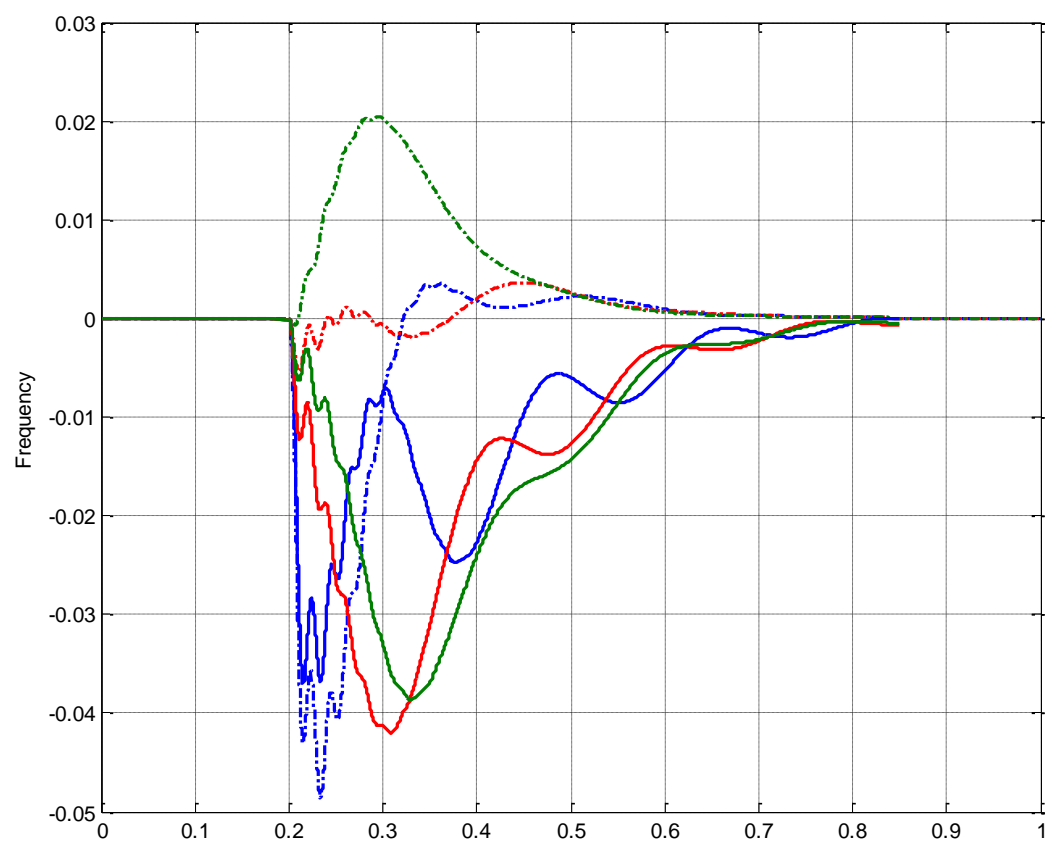

Figure 4.49: Frequency deviation with and without tie line error (Solid lines: with tie line error - Broken line: without tie line error)

\subsubsection{ROBUST Ho CONTROLLER}

In this section, the robust $\mathrm{H} \infty$ control design is proposed to solve the load frequency control problem. Similar to the previous control design, this robust control technique treats the load change as a disturbance, but it tends to minimize the worst case disturbance effects on the controlled variables rather than using it as feedback variables.

Over the past two decades, robust control theory has been useful when applied to control system designs that require robustness against possible disturbances such as parameter uncertainties, system modeling errors, plant and measurement noises, and external disturbances.

One major objective of robust control is synthesizing a controller that will guarantee the internal stability of the system when bounded perturbations are present. This proposed control design is different from other previous work. Specifically, the control design is cast into a linear matrix inequality (LMI) formulation, so that the powerful LMI control toolbox can be used to solve the constrained optimization problem to achieve the desired robustness.

Here, the robust $\mathrm{H} \infty$ control theory is adopted to design a controller for load frequency control. This wellknown approach provides the closed-loop system with robustness against bounded uncertainties. 


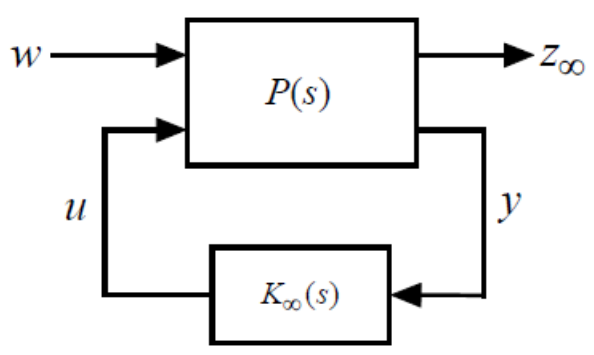

Figure 4.50: Close-loop system via robust $H \infty$ control.

Figure 4.50 shows a classical block diagram of the robust $\mathrm{H} \infty$ control problem. The objective is to design a control law $u$ based on the measured variables $y$ such that the effects of the disturbance, $w$, on the controlled variables $\mathrm{Z}_{\infty}$, which is expressed by the infinity norm of its transfer function $\left\|T_{z_{\infty} \omega}\right\|_{\infty}$ do not exceed a given value, $\gamma$, defined as guaranteed robust performance. In order to synthesize an $H \infty$ controller via LMI approach, state space realizations of the system $\mathrm{P}(\mathrm{s})$ and controller $\mathrm{K} \infty(\mathrm{s})$ are needed. They are given by equations $4.42-4.44$ and $4.45-4.46$ respectively. The State Space System Model is described below. [39]

$$
\begin{aligned}
& \Delta \dot{x}=A_{m g} \Delta x+B_{\omega} \omega+B_{m g} u \\
& y=C_{y} \Delta x+D_{y 1} \omega+D_{y 2} u \\
& z_{\infty}=C_{\infty} \Delta x+D_{\infty 1} \omega+D_{\infty 2} u
\end{aligned}
$$

(A, $\left.B_{m g}\right)$ is stabilizable, and $\left(A, C_{y}\right)$ is detectable. The State Space Controller Model below:

$$
\begin{aligned}
& \dot{\zeta}_{\infty}=A_{k \infty} \zeta_{\infty}+B_{k \infty} y \\
& u=C_{k \infty} \zeta_{\infty}+D_{k \infty} y
\end{aligned}
$$

Combining the above equations results in the following closed-loop system:

$$
\begin{aligned}
& \dot{x}_{c l}=A_{c l} x_{c l}+B_{c l} \omega \\
& z_{\infty}=C_{c l} x_{c l}+D_{c l} \omega
\end{aligned}
$$

Where

$$
\begin{aligned}
& x_{c l}=\left[\begin{array}{c}
x \\
\zeta_{\infty}
\end{array}\right] \\
& A_{c l}=\left[\begin{array}{cc}
A+B_{2} C_{k \infty} C_{y} & B_{2} C_{k \infty} \\
B_{k \infty} C_{y} & A_{k \infty}
\end{array}\right]
\end{aligned}
$$




$$
\begin{aligned}
B_{c l} & =\left[\begin{array}{c}
B_{1}+B_{2} C_{k \infty} D_{y 1} \\
B_{k \infty} D_{y 1}
\end{array}\right] \\
C_{c l} & =\left[\begin{array}{ll}
C_{\infty}+D_{\infty 2} D_{k \infty} C_{y} & D_{\infty 2} C_{k \infty}
\end{array}\right] \\
D_{c l} & =D_{\infty 1}+D_{\infty 2} D_{k \infty} D_{y 1}
\end{aligned}
$$

The following lemma relates Hळo control design to LMI.

Lemma1: the closed-loop RMS gain or the Hळ norm of the transfer function from w to $\mathrm{z} \infty,\left\|T_{z_{\infty} \omega}\right\|_{\infty}$, does not exceed $\gamma$, if and only if there exists a symmetric matrix $\mathrm{X} \infty$ such that [39]

$$
\begin{aligned}
& {\left[\begin{array}{ccc}
A_{c l} X_{\infty}+X_{\infty} A_{c l}{ }^{T} & B_{c l} & X_{\infty} C_{c l}{ }^{T} \\
B_{c l}{ }^{T} & -I & D_{c l}{ }^{T} \\
C_{c l} X_{\infty} & D_{c l} & -\gamma^{2} I
\end{array}\right]<0} \\
& X_{\infty}>0
\end{aligned}
$$

An optimal $\mathrm{H} \infty$ control design can be achieved by minimizing the guaranteed robust performance index, $\gamma$, subject to the constraints given by the matrix inequalities 4.54 and 4.55. The MATLAB's LMI control toolbox provides the function "hinflmi" to solve an $\mathrm{H} \infty$ control problem directly. This function returns the controller parameters, $\mathrm{K} \infty(\mathrm{s})$, as shown in 4.45 , with the optimal robust performance index $\gamma *$. The obtained controller is a dynamic type whose order is the size of the system matrix.

The system here has 47 state variables and 3 state variables related to the controller have also been added to the state equations so the system has totally 50 state variables.

$$
\begin{aligned}
& \Delta \dot{x}=A_{m g} \Delta x+B_{\omega} \omega+B_{m g} u \\
& y=C_{y} \Delta x+D_{y 1} \omega+D_{y 2} u \\
& z_{\infty}=C_{\infty} \Delta x+D_{\infty 1} \omega+D_{\infty 2} u \\
& z_{\infty}=\left[\begin{array}{c}
A C E \\
\int A C E
\end{array}\right] \Rightarrow \text { for one area } s y s: z_{\infty}=\left[\begin{array}{c}
\Delta \omega_{i} \\
\int \Delta \omega_{i}
\end{array}\right]
\end{aligned}
$$




$$
z_{\infty}=\left[\begin{array}{c}
\Delta \omega_{i} \\
\int \Delta \omega_{i}
\end{array}\right]=\left[\begin{array}{c}
\Delta \omega_{1} \\
\Delta \omega_{2} \\
\Delta \omega_{3} \\
\int \Delta \omega_{1} \\
\int \Delta \omega_{2} \\
\int \Delta \omega_{3}
\end{array}\right]=\left[\begin{array}{c}
\Delta \omega_{n 1}-m_{p 1} \Delta p_{1} \\
\Delta \omega_{n 2}-m_{p 2} \Delta p_{2} \\
\Delta \omega_{n 3}-m_{p 3} \Delta p 3 \\
\int \Delta \omega_{1} \\
\int \Delta \omega_{2} \\
\int \Delta \omega_{3}
\end{array}\right]=\left[\begin{array}{c}
u_{1}-m_{p 1} x_{2} \\
u_{2}-m_{p 2} x_{15} \\
u_{3}-m_{p 3} x_{28} \\
x_{48} \\
x_{49} \\
x_{50}
\end{array}\right]=C_{\infty} \Delta x+D_{\infty 2} u
$$

Figure 4.51 shows the results related to frequency deviation with PI and $\mathrm{H} \infty$ controller.

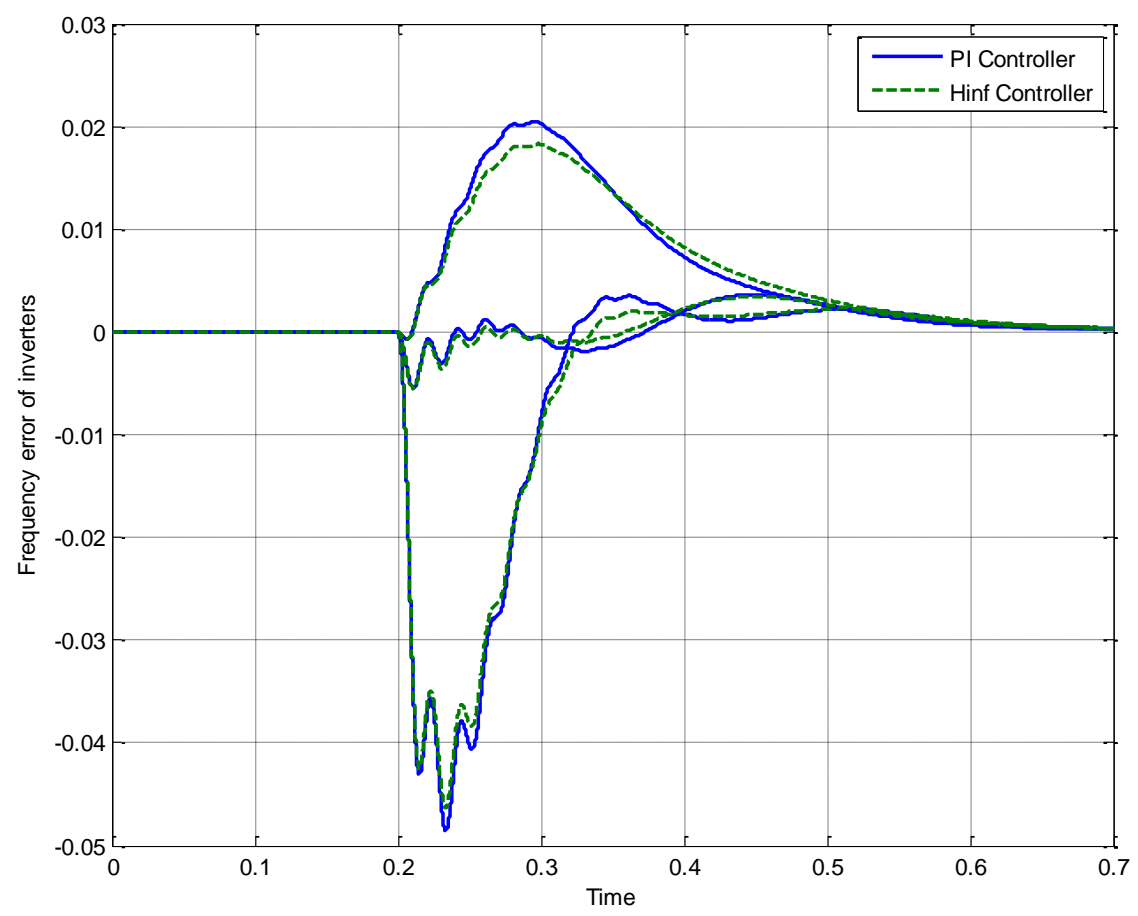

Figure 4.51: Frequency deviation with and without $H \infty$ (Solid lines: with PI controller-Broken line: with $H \infty$ controller)

\subsection{MODEL VERIFICATION}

The studied microgrid in section 4.4.1 is modeled by MATLAB based on the equations discussed in the modeling chapter (chapter three). The purpose is verifying the accuracy of the modeling implemented in MATLAB by comparing it to the PSCAD detailed modeling.

As previously stated, the main components of the system are the PV generator, the battery energy storage, the load demand, the microgrid and the power electronics components. The PV and battery are connected to the microgrid through a DC/AC inverter. Controlling the inverters in a microgrid is a key point. In this 
modeling, the frequency of a microgrid has been controlled by sending an appropriate control signal to the inverters.

Considering the configuration of the system used, the microgrid is divided into two areas. Figure 4.31 shows the way we made the areas in this system. In fact, for the third case, we have two assigned areas where each of them is supposed to take care of its own frequency, so whenever the load is changed in each area, that particular area does its best to control the frequency.

In this case, there is an imaginary tie line trying to have a predetermined value for its power flow. The error of tie-line power from the predetermined value is added as ACE to the set-point of PV/Batt system. So, the important term defining our new ACE is a coefficient of tie line error.

\subsubsection{DETAILED MODEL (IN PSCAD) VS AVERAGE MODEL (IN MATLAB)}

The understudied microgrid is simulated in PDCSAD environment with the detailed component to investigate the accurate result of having a virtual tie line error. However, in order to verify the equationbased modeling (the average model) and compare it with the detailed model, a similar system with average components is implemented in MATLAB software. The main elements affected by the average model are power electronic devices such as inverters. By neglecting the switching action of these converters and assuming the ideal functionality of the three legs inverter, their detailed model is simplified significantly. Below, some of the results have been compared with the detailed and average model. First, the frequency deviation in both simulations are shown, then the power produced by battery and finally the tie-line power is illustrated.
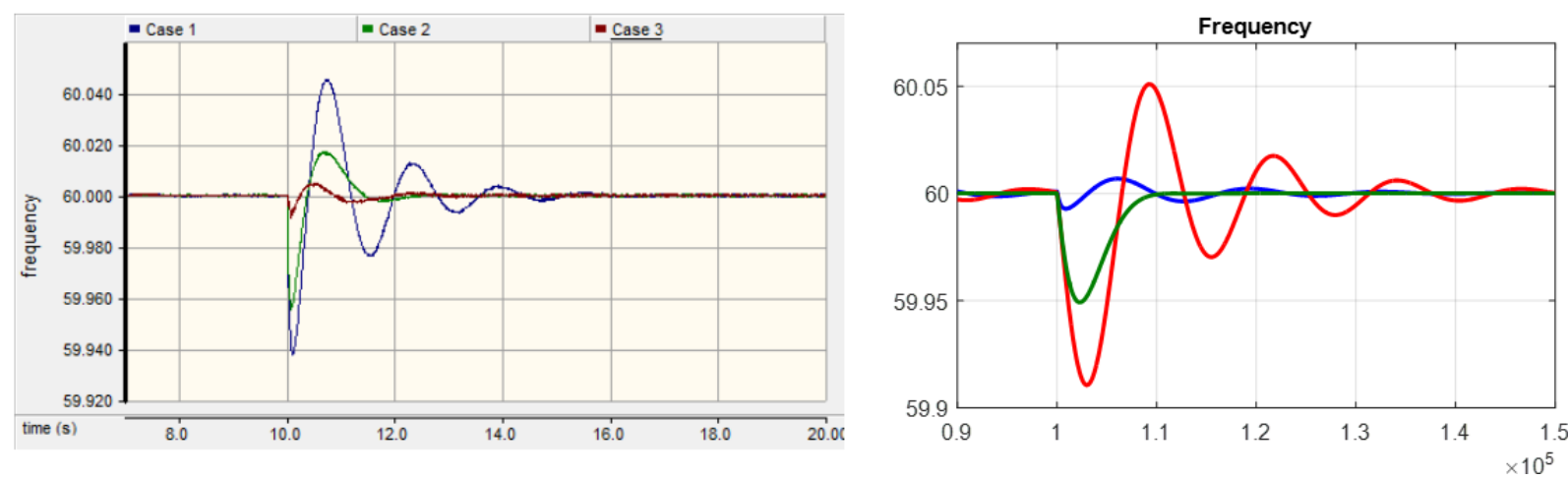

Figure 4.52: Frequency error for three scenarios (right and left figures are related to the average and detailed model) 

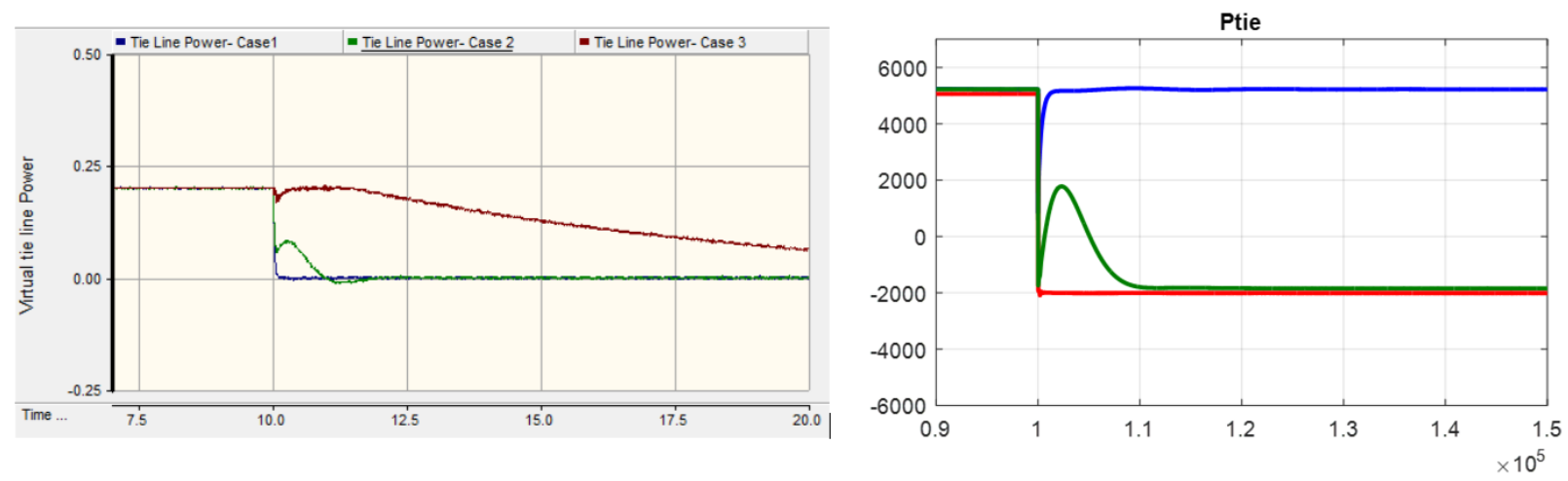

Figure 4.53: Tie line power for three scenarios (right and left figures are related to the average and detailed model respectively)
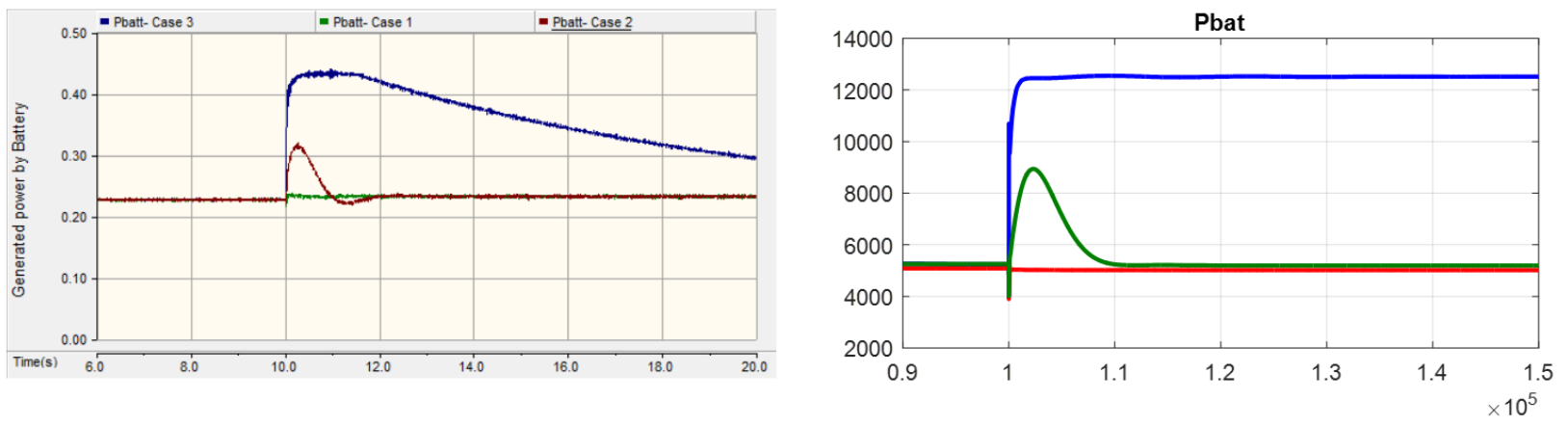

Figure 4.54: Power generated by battery for three scenarios (right and left figures are related to the average and detailed model respectively)

The reason behind the differences are stated below:

The main difference between these results is because of considering the effect of SOC of battery in the detailed model and not considering that in the average model.

Other than that, the rotating machine and battery used in these simulations are the default model available in related software's library. So, some of the parameters are not exactly the same, resulting in some differences in the figures.

$>$ The PI controller used for controlling frequency in the PSCAD and MATLAB software are not the exact same one. 


\section{Chapter 5}

\section{Control of Microgrid Voltage Profile using Consensus-Based Algorithm}

One of the important issues about microgrids is voltage control and reactive power sharing. As it was discussed during the literature survey, there are many recent works concentrating on this problem. Droop control is mostly used as a primary control to share the reactive power among DGs based on some criteria, such as rating; however, the secondary control is also needed to complete this task and compensate for the remaining voltage error. There are different solutions for secondary control; however, a consensus-based distributed voltage control is proposed here to guarantee proper reactive power sharing among DGs.

In spite of most other related communication-based control concepts, the proposed approach will only require sparse communication among DGs, which means central controller or all-to-all communication among DGs is no longer required.

The chosen protocol for exchanging data among agents is consensus protocol, which helps agents to reach a global awareness about voltage and power sharing situations. 


\section{Consensus Problem in Networks of Agents}

Consensus problems have a long history in the field of computer science [38]. In many applications, groups of agents need to agree upon certain quantities of interest. Such quantities might or might not be related to the motion of the individual agents. As a result, it is important to address agreement problems in their general form for networks of dynamic agents with directed information flow under link failures and the creation of new ones (i.e., switching network topology).

Whenever the nodes of a network are all in agreement, the common value of all nodes is called the group decision value. We are interested in distributed solutions of the consensus problem in which no node is connected to all other nodes. There are special cases such as average-consensus, max-consensus and minconsensus, which have a broad application in distributed decision making for multi-agent systems.

\section{ApPlication of Consensus Solution fOR SECONDARY Voltage ConTrol}

Each bus in a microgrid has some condition on its voltage such as minimum and maximum thresholds. Usually, the main constraint needed to be considered is the lowest possible voltage at the bus. However, the highest possible voltage is also a constraint as well. Moreover, the average voltage of microgrid buses can be a consideration. In order to ensure that the average of all voltage amplitude in the network is equivalent to the nominal voltage amplitude, or at least in a nominal range, the average consensus solution can be used. The other two consensus solution also can be used to ensure all buses individually have a voltage in acceptable ranges.

A good point to consider is that in any region, there may be a bus that is more critical, meaning that by satisfying the constraint for that specific bus can ensure the other buses are also in safe voltage ranges. These critical buses are called pilot voltage buses and by finding these points, the communication network can be reduced significantly. 


\subsection{AbSTRACT}

A decentralized multi-agent-based voltage control strategy is proposed and investigated in this chapter on an islanded microgrid with communication constraints, where each agent can only communicate with a few neighboring agents, not all of them. The connection between neighbors is defined by the network design problem to achieve a trade-off between the communication cost and the agreement time delay for consensus algorithm. After designing an appropriate graph for the network of agents, the average consensus algorithm is used to accurately share global information, such as the average voltage of microgrid's buses, in a decentralized way. Depending on the discovered global information, the cooperative average voltage control strategy, which involves the secondary voltage control, is executed to achieve a cooperative voltage regulation. Simulation results indicate that the proposed average voltage control approach can guarantee the consensus and coordination of the distributed agents and maintain the voltage stability of islanded microgrids and bring back the average voltage to the proper value.

This chapter introduces a decentralized multi-agent system without an MGCC for voltage control of an islanded microgrid to overcome the shortcomings related to the centralized solution. The advantages of the decentralized scheme include the ability to survive uncertain disturbances and decentralized data updating, which leads to efficient information sharing, eventually causing a faster decision making process and operation [16], [17]. Olfati-Saber and Murray in [18] introduced the average consensus algorithm, which relies on local information from the agents and solves the consensus problems of the decentralized multiagent system. Further, theoretical extensions of this work were presented in [19]-[22] and a recent survey can be found in [23].

In this study, an average consensus algorithm (ACA) is proposed to guarantee that required information is shared in a distributed way, where agents can only communicate with a few of their neighboring agents based on the communication network design. In addition, the impacts of communication time delays are further discussed in this study.

The voltage control of microgrids is a two-layered cooperative control using the droop controller in the primary layer and the ACA-based voltage regulation in the secondary layer, which together enables microgrids to maintain the voltage stability as well as the voltage quality by smoothing the voltage profile. As mentioned earlier during the frequency control discussion, the primary voltage control is implemented through the generating units as well as energy storage devices while the secondary ACA-based voltage regulation is done only by the participation of generating units so that the power output of the energy storage devices are regulated to the initial state. The required global information of the system can be discovered only by communication of the neighboring agents according to the chosen ACA. (The network design problem will decide about neighboring of agents.) As a result, the control modes and corresponding decision making for each agent can be determined. 
To evaluate the proposed voltage control strategy, a simulation system was designed and the corresponding dynamic simulation model based on MATLAB/Simulink was developed. Simulation results are presented and discussed to illustrate the dynamic performance of the proposed control strategy.

In order to achieve an optimum solution for decentralized and multi-agent-based voltage control of an islanded microgrid, the following steps need to be taken:

1- The best network structure for multi-agents should be decided: this topology will be used for implementing the average consensus algorithm and it has the direct effect of the agreement time delay of the consensus algorithm, so by considering the communication cost as a constraint, the best topology that gives the lowest agreement time can be decided.

2- The updating matrix should be calculated: there are different methods for forming the updating matrix and they have different speeds of convergence. So, these methods should be compared and one of them should be selected to be used for the average consensus protocol.

3- After deciding the network structure and the updating matrix, the number of iteration needed for the agreement and subsequently the time interval needed for reaching this agreement has to be calculated.

4- The decision-making algorithm should be introduced and examined by considering the agreement delay interval.

\subsection{OPTIMUM NETWORK STRUCTURE FOR MULTI AGENTS}

\subsubsection{CONSENSUS Problem ON A Graph}

Here in this section, the consensus protocol for continuous and discrete systems is presented. The protocol is supposed to solve network agreement problem. There are different parameters that need to be defined to have an optimal consensus problem. In the following, the procedure of finding the optimal solution is discussed.

Equation 5.1 and 5.2 show the agent's dynamic in continuous and discrete systems and equation 5.3 is the linear consensus protocol applied to them, which assumes that there is not any communication delay. Here $x(t)$ represent the state variables and $u(t)$ is the control input. $a_{i j}$ is representing the elements of adjacency matrix, which is described later around. [38]

$$
\begin{aligned}
& \dot{x}_{i}(t)=u_{i}(t) \\
& x_{i}(k+1)=x_{i}(k)+\varepsilon u_{i}(k)
\end{aligned}
$$




$$
u_{i}=\sum_{v_{j} \in N_{i}} a_{i j}\left(x_{j}-x_{i}\right)
$$

As seen in figure 5.1, $\mathrm{N}_{\mathrm{i}}$ is the neighboring set of node $v_{i}$ and $a_{i j}$ (each node is representing an associated agent) is the element of Adjacency Matrix, $\mathrm{A}=\left[\mathrm{a}_{\mathrm{ij}}\right]$; if there is a connection link between node $v_{\mathrm{i}}$ and node $v_{j}$, then $a_{i j}$ is nonzero, otherwise it would be zero. If the nonzero elements of Adjacency matrix are something different than one, then it would also be called Updating Matrix, W. (The nonzero elements of Updating matrix can be defined based on different methods, which would be discussed later.) In order to achieve an agreement in the minimum period of time, it is important to design the best adjacency matrix. It is obvious that having more communication links can help the algorithm to reach the agreement in a shorter interval; however, at the same time, it will cause more communication cost.

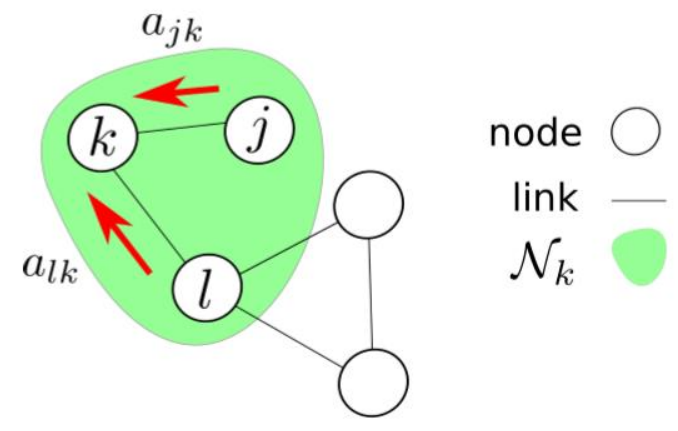

Figure 5.1: Neighbors and adjacency element of a graph

It is crucial to define the communication cost of the multi-agent network before going forward. The communication cost equation of a graph with adjacency matrix A is shown below in 5.4. It should be considered that we need to keep the communication cost limited.

$$
C=\sum_{i, j=1}^{n} \operatorname{sgn}\left(a_{i j}\right)
$$

Equation 5.4 shows that there would be a cost for having any communication link between two nodes. It also emphasizes that a two-way communication link will cost twice as much as a one way link. An important problem in the network layer is the network design problem, which means choosing the appropriate $a_{i j}$ for the adjacency matrix considering a bounded communication cost while optimizing a specific performance index. In another words, it means that neighbors should have direct connection links between them.

Using the mentioned consensus protocol, the state of a continuous system of integrator will change according to the following linear system described as 5.5:

$$
\dot{x}(t)=-L x(t)
$$




$$
L_{i j}= \begin{cases}\sum_{k=1, k \neq i}^{n} a_{i k} & i=j \\ -a_{i j} & i \neq j\end{cases}
$$

Here in these equations, $\mathrm{L}$ is called the graph Laplacian, which can be calculated from the adjacency matrix elements. The eigenvalues of the graph Laplacian matrix are important for determining the stability and convergence of the consensus algorithm.

For those networks having a discrete model, the network dynamic can be described as 5.7-5.8:

$$
\begin{aligned}
& x(k+1)=P_{\varepsilon} x(k) \\
& P_{\varepsilon}=I-\varepsilon L \quad \varepsilon \in\left(0, \frac{1}{d_{\text {max }}}\right)
\end{aligned}
$$

Assuming $\mathrm{d}_{\max }=\max \mathrm{L}_{\mathrm{ii}}, \mathrm{P}_{\varepsilon}$ would be a non-negative and stochastic matrix, which is called Perron Matrix. Perron matrix can be formed based on equation 5.8 by using Laplacian matrix.

Each node in a directed graph has in-degree and out-degree values. In fact, the out-degree of a node in a graph with $0-1$ adjacency elements is the number of immediate neighbors related to that particular node. Out-degree and in-degree of each node can be calculated as shown in 5.9-5.10.

$$
\begin{aligned}
& \operatorname{deg}_{\text {out }}\left(v_{i}\right)=\sum_{j=1}^{n} a_{i j} \\
& \operatorname{deg}_{\text {in }}\left(v_{i}\right)=\sum_{j=1}^{n} a_{j i}
\end{aligned}
$$

In order to define an appropriate criterion for comparing the convergence speed, the Degree Matrix, $\Delta$, is needed to be defined. The degree matrix is diagonal, which uses the out-degree value of each node for its diagonal elements. This matrix is shown in 5.11 .

$$
\Delta=\left[\begin{array}{cccc}
\operatorname{deg}_{\text {out }}\left(v_{1}\right) & 0 & 0 & 0 \\
0 & \operatorname{deg}_{\text {out }}\left(v_{2}\right) & 0 & 0 \\
0 & 0 & \ddots & 0 \\
0 & 0 & 0 & \operatorname{deg}_{\text {out }}\left(v_{n}\right)
\end{array}\right]
$$

The graph Laplacian of a network can be calculated through equation 5.12. According to the definition of a Laplacian matrix, the sum of each row is equal to zero, so there would be a zero eigenvalue in the set of eigenvalues for sure. As a result of this zero eigenvalue and identical nonzero elements of L, rank (L) would be less or equal to $n-1$. 


$$
\ell(G)=L=\Delta-A
$$

\section{1) Undirected and directed graph properties}

In order to solve the consensus problem in a network of agents and to reach an agreement between them, some properties of the graphs should be investigated. The first property would be the connectivity.

a) Strongly connected graph

A digraph (directed graph) is called strongly connected, SC, if and only if each two separate nodes can be connected through a path of edges with correct direction. This property has a strong relation to the rank of the graph. There is a theorem in [38] that proves if a digraph is strongly connected, then $\operatorname{rank}(L)=n-1$. However, for an undirected graph, the aforementioned theorem will also remain correct in the opposite direction. In another words, if the rank of an undirected graph is n-1, it can be concluded that it is strongly connected. This is not necessarily correct for a digraph.

Notation 1: considering a network of integrators, where each node applies the consensus protocol; then the protocol will solve the consensus problem if the digraph is strongly connected. However, it does not guarantee that the group decision value would be equal to the average of initial values. It will be discussed later that another constraint should be satisfied in order to reach an average consensus agreement between nodes.

Notation 2: A sufficient condition for a graph to reach average consensus agreement is that $\sum_{i=1}^{n} u_{i} \equiv 0$. This condition will automatically hold for any undirected graph but for diagraph, it is not generally true, unless the digraph is balanced. The balance property of a graph would be investigated afterward.

\section{b) Balanced graph}

A graph is called balanced if and only if all the included nodes are balanced and being a balanced node means that the out-degree and in-degree of that node is equal. So, the equation 5.13 will hold for any balanced graph:

$$
\text { in }-\operatorname{deg} \operatorname{ree}\left(v_{i}\right)=\text { out }-\operatorname{deg} r e e\left(v_{i}\right) \quad \forall i
$$

It should be noticed that any undirected graph is automatically balanced. Table 5.1 shows some balanced and unbalanced digraphs. 
TABLE 5.1

BALANCED AND UNBALANCED DIGRAPH EXAMPLE

\begin{tabular}{|l|l|}
\hline Unbalanced Digraph & balanced Digraph \\
\hline
\end{tabular}

\subsubsection{Performance Measurement of Consensus Protocol}

The consensus protocol that is going to be applied to the network of agents needs to have an appropriate performance measurement criteria. It was discussed previously that any balanced digraph will agree on the average of initial values, which means that the agreement value is an invariant quantity. Considering Agreement value, $\alpha$ - equal to the average of initials - the state of nodes can be described as equation 5.14, where $\delta$ is referred to as a disagreement vector and satisfies $\sum_{i} \delta_{i}=0$.

$$
x=\alpha I+\delta
$$

The dynamic of $\delta$ is described in 5.15. It is obvious that the damping speed of $\delta$ is related to the eigenvalues of L. So, Algebraic connectivity, which is an important criterion associated with a graph, has been defined to describe that. Fiedler established a connection between this criterion and the second largest eigenvalue of Laplacian matrix of an undirected graph, $\lambda_{2}(\mathrm{~L})$. Because of that, it is called Fiedler eigenvalue. However, for expanding this idea to algebraic connectivity of digraph, this notation was then used in [40] by defining the mirror operation on digraphs $(G)$, which resulted in an undirected graph $(\widehat{G})$ that has the same graph Laplacian properties. So, in order to measure this speed, first, the mirror graph operation needs to be discussed because the Laplacian disagreement of digraphs, $\mathrm{L}$, is not necessarily nonnegative.

$$
\dot{\delta}=-L \delta
$$




\section{1) Mirror Graph Operation}

Assume that $G$ is a weighted digraph. Graph $\widehat{G}$ will be an undirected graph having the same set of nodes as $\mathrm{G}$ while the set of edges related to it consists of the edge set of $\mathrm{G}$ plus a set of the reversed direction edges. The adjacency matrix of graph $\hat{G}$ is a symmetric matrix with element shown in 5.16:

$$
\widehat{a}_{i j}=\widehat{a}_{j i}=\frac{1}{2}\left(a_{i j}+a_{j i}\right)
$$

Theorem [38]: When considering digraph $\mathrm{G}$ with adjacency matrix $\mathrm{A}$ and $\hat{G}$ as its undirected mirrored graph with adjacency element described in equation 5.16, the Laplacian matrix of $\widehat{G}$ would be $L_{S}=\left(L+L^{T}\right) / 2$ if and only if $G$ is balanced. Moreover, if $G$ is balanced, the Laplacian disagreement of digraph $G$ and undirected graph $\widehat{G}$ would be equal.

After discussing mirrored graph Laplacian, the performance criterion can be presented. In fact, the performance of the consensus algorithm applied to a digraph (showing the worst case speed of reaching an agreement) can be measured by the second largest eigenvalue or so-called Fiedler eigenvalue of $L_{s}$. This criterion is called Graph Connectivity (or Algebraic Connectivity), $k$, and can show how fast the disagreement vector would vanish.

$$
\begin{aligned}
& \|\delta(t)\| \leq\|\delta(0)\| e^{-k t} \\
& k=\lambda_{2}(\widehat{G})
\end{aligned}
$$

Based on the observation, $\lambda_{2}$ is usually a large value for dense graphs and small for sparse graphs. This is the reason behind calling it graph connectivity. So, it means that having a graph with a larger set of edges can reach the agreement faster. However, at the same time, it increases the cost function related to the graph.

\section{2) Tradeoff between communication cost and connectivity}

For a strongly connected digraph with a large number of edges, the graph connectivity is usually high, which represent a high performance of consensus protocol. However, it obviously increases the complexity and communication cost related to the network. So, there is a tradeoff between the graph connectivity criterion and the communication cost, which leads to design network problem that is discussed and investigated in 5.2.3.

\subsubsection{APPROPRIATE NETWORK DESIGN CONSIDERING THE DEFINED CRITERIA}

When designing a network of agents, there are some criteria that need to be considered. The first one is the communication cost, which can be approximated based on the number of one-way communication links 
provided for the network. This cost is limited so there would be a maximum cost value for the network of $n$ nodes. Based on the application that the network is going to be used for, the minimum required graph connectivity can be defined as well. Finally, based on the provided maximum communication cost, $C_{\max }$, and the required minimum graph connectivity, $k_{\min }$, a possible structure can be designed for a graph of $\mathrm{n}$ nodes by the use of the flowchart shown in figure 5.2.a.

Based on this flowchart, $C_{\max }$ and $k_{\min }$ would be the input of the program, which is defined based on the application requirement and limitations. Then a random adjacency matrix with $0-1$ elements will be formed considering the cost threshold. The matrix will be then examined to see if it is balanced and strongly connected respectively. If any of these two matrix properties are violated, then another random matrix will be chosen and the process goes to the first step.

After finding an appropriately balanced and strongly connected adjacency matrix, the graph and mirrored graph Laplacian would be calculated to find out the graph connectivity. Then if the calculated graph connectivity satisfies the minimum requirement, it means that a proper network topology has been designed; otherwise, it should go to the start.

In order to show the effectiveness of the program for solving the network design problem, the solutions to some cases are shown afterward. Table 5.2 describes the input value for the first example. It is been assumed that there are 10 nodes in the network and the maximum communication cost is 12 while the minimum required graph connectivity is 0.25 . The result of this example is demonstrated in figure 5.3.

The tables related to the second and third examples are also shown in table 5.3 and table 5.4 respectively and the solutions to these examples are shown in figure 5.4 and 5.5. In these examples, the process stops as soon as it finds a proper answer.

\subsubsection{OPTIMUM NETWORK STRUCTURE}

Designing a proper graph to satisfy the network criteria, using the mentioned flowchart in section 5.2.3 may propose a suitable topology for the system network but it does not mean that it is optimum. So, in order to find the optimum solution, an objective function is introduced in 5.18. The mentioned flowchart will be used as an inside block and another loop will be added to the program to find out the optimum solution by calculating the introduced objective function and the related flowchart is shown in 5.2.b. In 5.18, $C$ is communication cost, $k$ is the graph connectivity and $\alpha$ is the defined connectivity gain weight. In these study $\alpha$ is set as 4 .

$$
o b f=C+\frac{\alpha}{k}
$$




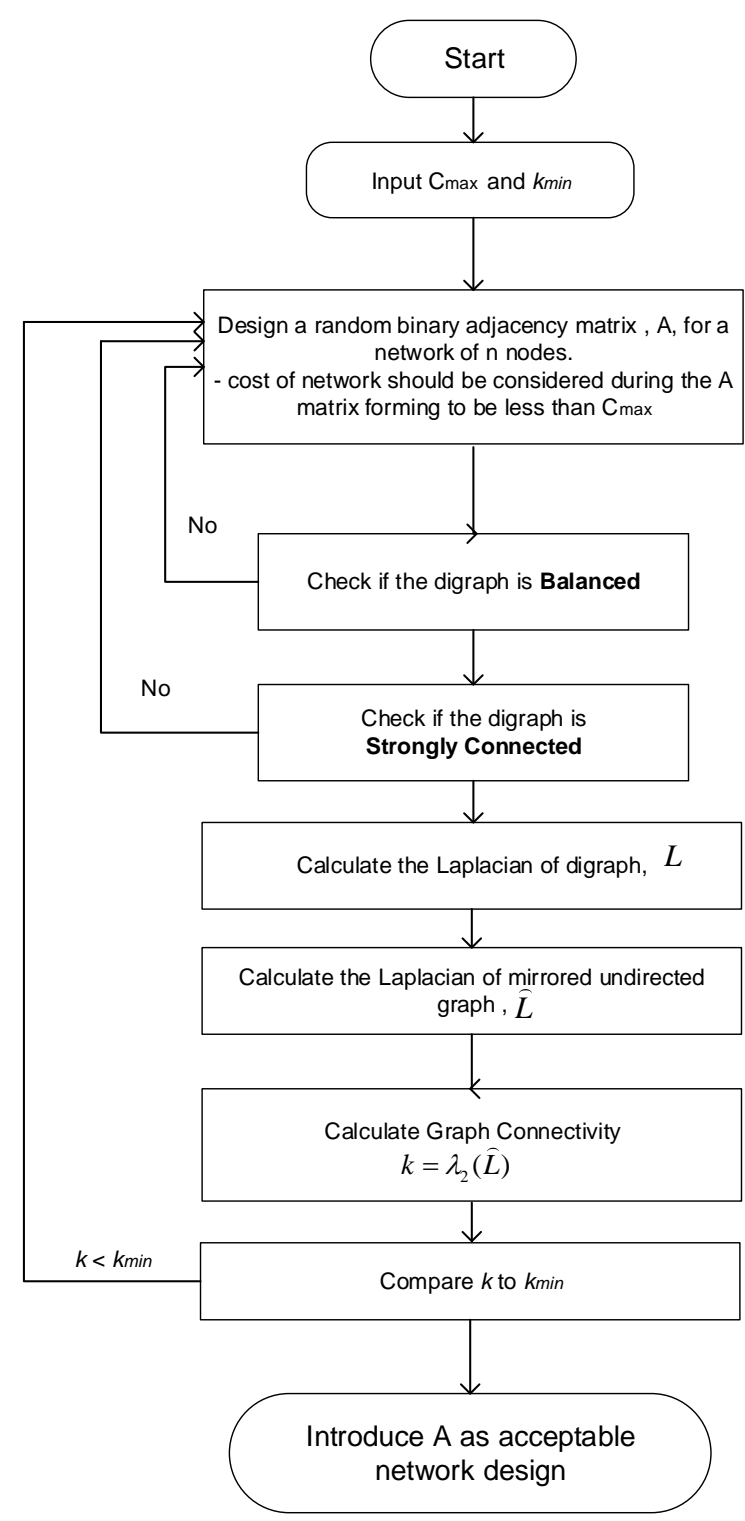

Figure 5.2.a: Flowchart related to the proper random network design problem of consensus protocol

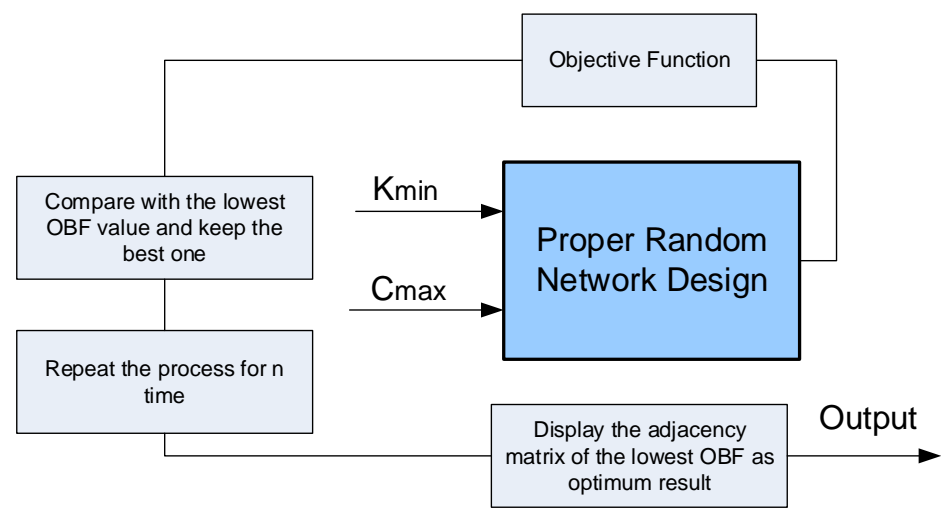

Figure 5.2.b: Flowchart related to the optimum network design 
TABLE 5.2

EXAMPLE 1

\begin{tabular}{|c|c|c|c|}
\hline \multirow{2}{*}{ Input Criteria } & No. of nodes & $\begin{array}{c}\text { Max Digraph Cost } \\
\text { Cmax }\end{array}$ & $\begin{array}{c}\text { Min Digraph Connectivity } \\
\text { Kmin }\end{array}$ \\
\cline { 2 - 4 } & $\mathbf{1 0}$ & $\mathbf{1 2}$ & $\mathbf{0 . 2 5}$ \\
\hline $\begin{array}{c}\text { Acceptable } \\
\text { Aesigned Network }\end{array}$ & $\mathbf{0 b f}$ & Digraph Cost & Digraph Connectivity \\
\hline (B) & $\mathbf{2 7 . 4 2 0 0}$ & $\mathbf{C}$ & $\mathbf{K}$ \\
\hline Optimum Network & Obf opt & Digraph Cost & Digraph Connectivity \\
(after 100 iteration) & $\mathbf{2 5 . 6 5 6 9}$ & $\mathbf{C}$ & $\mathbf{0 . 2 5 9 4}$ \\
\hline
\end{tabular}

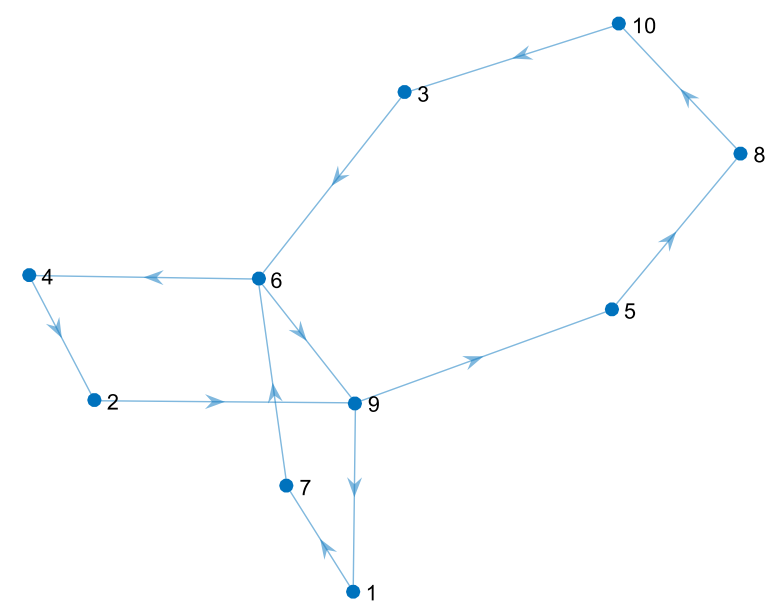

Figure 5.3: Solution for example 1- (A: Acceptable Designed Network)

TABLE 5.3

EXAMPLE 2

\begin{tabular}{|c|c|c|c|}
\hline \multirow[t]{2}{*}{ Input Criteria } & No. of nodes & $\begin{array}{c}\text { Max Digraph Cost } \\
\text { Cmax }\end{array}$ & $\begin{array}{c}\text { Min Digraph Connectivity } \\
\text { Kmin }\end{array}$ \\
\hline & 10 & 12 & 0.2 \\
\hline \multirow{2}{*}{$\begin{array}{c}(\mathrm{A}) \\
\text { Acceptable } \\
\text { Designed Network }\end{array}$} & obf & $\begin{array}{c}\text { Digraph Cost } \\
\text { C }\end{array}$ & $\begin{array}{c}\text { Digraph Connectivity } \\
\text { K }\end{array}$ \\
\hline & 30.2462 & 12 & 0.2192 \\
\hline \multirow{2}{*}{$\begin{array}{c}\text { (B) } \\
\text { Optimum Network } \\
\text { (after } 100 \text { iteration) }\end{array}$} & Obf opt & $\begin{array}{c}\text { Digraph Cost } \\
\text { C }\end{array}$ & $\begin{array}{c}\text { Digraph Connectivity } \\
\text { K }\end{array}$ \\
\hline & 25.6569 & 12 & 0.2929 \\
\hline
\end{tabular}




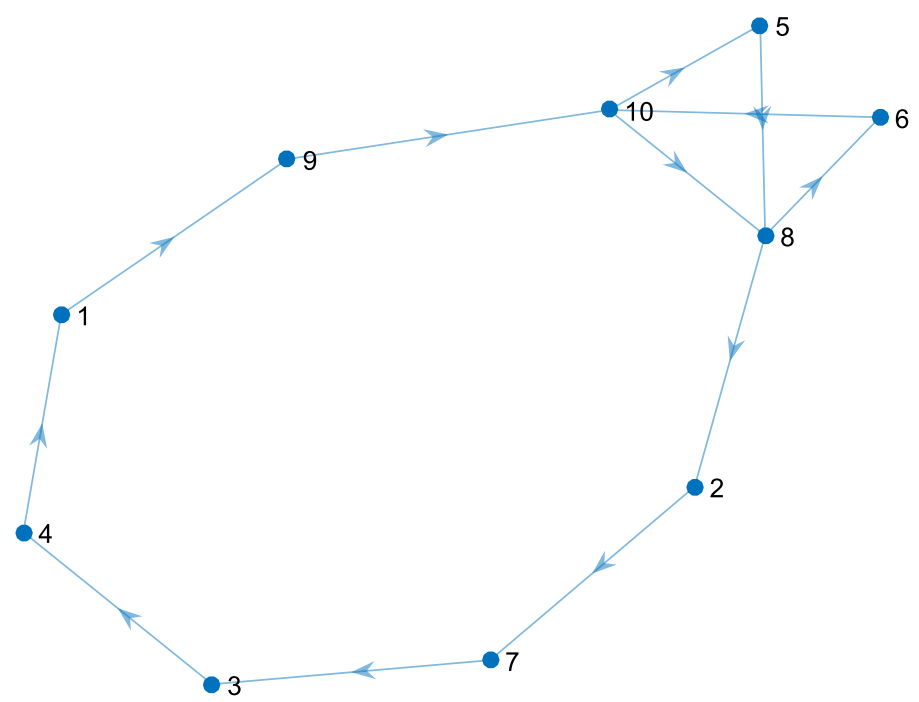

Figure 5.4: Solution for example 2- (A: Acceptable Designed Network)

TABLE 5.4

EXAMPLE 3

\begin{tabular}{|c|c|c|c|}
\hline \multirow{2}{*}{ Input Criteria } & No. of nodes & $\begin{array}{c}\text { Max Digraph Cost } \\
\text { Cmax }\end{array}$ & $\begin{array}{c}\text { Min Digraph Connectivity } \\
\text { Kmin }\end{array}$ \\
\cline { 2 - 4 } & $\mathbf{1 0}$ & $\mathbf{1 5}$ & $\mathbf{0 . 3 2}$ \\
\hline (A) & obf & Digraph Cost & Digraph Connectivity \\
Acceptable & $\mathbf{C}$ & $\mathbf{K}$ \\
\hline Designed Network & $\mathbf{2 7 . 3 2 5 0}$ & $\mathbf{1 5}$ & $\mathbf{0 . 3 2 4 5}$ \\
\hline (B) & Obf opt & Digraph Cost & Digraph Connectivity \\
\hline Optimum Network & $\mathbf{C}$ & $\mathbf{K}$ \\
\hline (after 100 iteration) & $\mathbf{2 3 . 6 8 1 0}$ & $\mathbf{1 5}$ & $\mathbf{0 . 4 6 0 8}$ \\
\hline
\end{tabular}

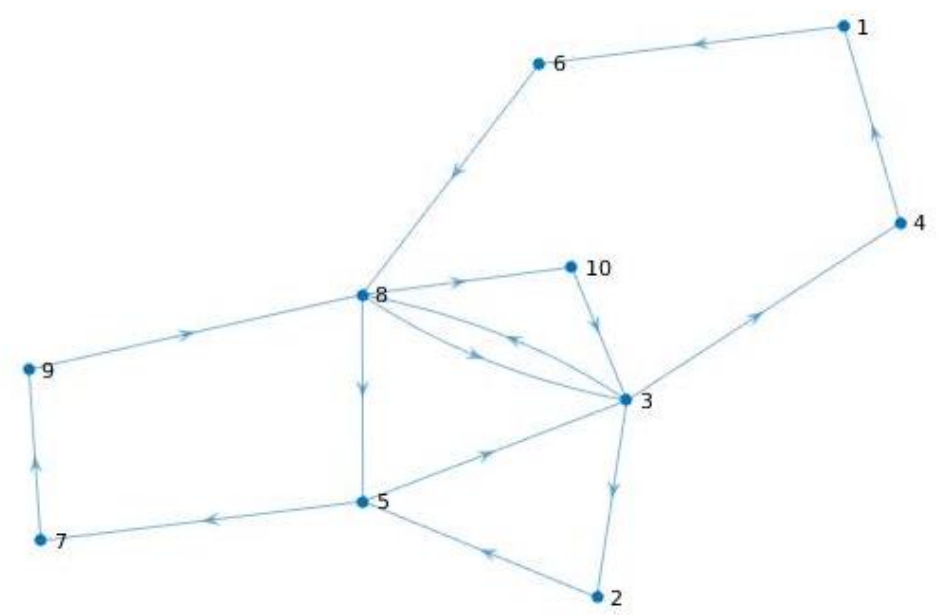

Figure 5.5.a: Solution for example 3- (A: Acceptable Designed Network) 


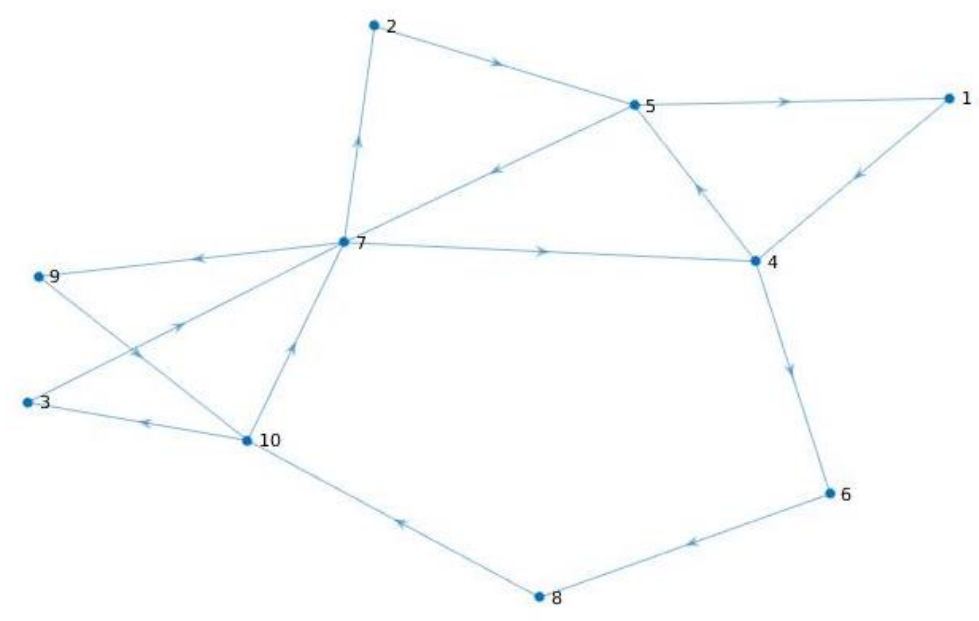

Figure 5.5.b: Solution for example 3- (B: Optimum Network)

\subsection{UPDATING MATRIX}

The coefficient for information exchange between the neighboring agents firstly depends on the structure of the network so the updating matrix would change according to the topology. After deciding the network structure, the updating matrix should be calculated. There are different methods for forming the updating matrix and they have different speeds of convergence. So, these methods should be compared and one of them should be selected to be used for the average consensus protocol.

Based on what was said earlier, $V_{b}$ (bus voltages) can then converge to the new average value. Consequently, the global information of average bus voltages $\mathrm{V}_{\text {bavg }}$ is shared in a distributed way to all decentralized agents for decision making. Finally, based on the shared global information achieved by proposed ACA, the ACA-based voltage controller can be implemented to achieve a cooperative voltage recovery.

\subsubsection{COEFFICIENTS SETTING OF UPDATING MATRIX}

An average consensus algorithm is a subdivision of distributed averaging algorithm in which all the nodes are being activated at each time slot $t$ to communicate with their neighbors and update their current state. There are different average consensus strategies for the choice of the weights. This choice can directly affect the number of steps needed for convergence. In another words, for a constant network topology, the selection of updating matrix coefficient can affect the agreement time delay significantly. Thus, it is needed to look for the best adjusting coefficient for the updating matrix to achieve near optimal performance. 


\section{1) Uniform weights}

One of the common and easy choices for setting the updating matrix elements is Uniform method. According to the method, the weights are fixed and can be calculated based on 5.19. $n$ is showing the number of nodes.

$$
a_{i j}=\left\{\begin{array}{lr}
\frac{1}{n} & j \in N_{i} \\
1-\sum_{j \in N_{i}} \frac{1}{n} & j=i \\
0 & \text { otherwise }
\end{array}\right.
$$

\section{2) Maximum-Out Degree weight}

Let us call $d$ the maximum out-degree of the network.

$$
d=\underset{i \in v}{\operatorname{Max}}\left\{\operatorname{deg}_{\text {out }}\left(v_{i}\right)\right\}
$$

Every node weighs the state of its neighbor $\frac{1}{d+1}$ and gives to its own state the remaining weight. Therefore, the coefficient would be formed as in 5.21 .

$$
a_{i j}=\left\{\begin{array}{lr}
\frac{1}{d+1} & j \in N_{i} \\
1-\frac{\operatorname{deg}_{\text {out }}\left(v_{i}\right)}{d+1} & i=j \\
0 & \text { otherwise }
\end{array}\right.
$$

\section{3) Metropolis weights}

Metropolis method is another option that was introduced in [19] to improve the speed of the uniform method. One of the positive points about metropolis method is that it is adaptive toward the changes in system configurations. The weight obtained by this method is described by equation 5.22 .

$$
a_{i j}=\left\{\begin{array}{lr}
\frac{1}{\left[\operatorname{Max}\left(n_{i}+n_{j}\right)+1\right]} & j \in N_{i} \\
1-\sum_{j \in N_{i}} \frac{1}{\left[\operatorname{Max}\left(n_{i}+n_{j}\right)+1\right]} & j=i \\
0 & \text { otherwise }
\end{array}\right.
$$

\section{4) Mean Metropolis weight}

This is an improved algorithm proposed in [41], which is inspired by the Lyapunov stability analysis. Similar to the Metropolis method, this new algorithm has the properties of stability and adaptability. The 
improvement is with the near optimal converging speed, which will be demonstrated in the result section. The updating rule of this method is presented in 5.23.

$$
a_{i j}=\left\{\begin{array}{lr}
\frac{2}{\left(n_{i}+n_{j}+\varepsilon\right)} & j \in N_{i} \\
1-\sum_{j \in N_{i}} \frac{2}{\left(n_{i}+n_{j}+\varepsilon\right)} & j=i \\
0 & \text { otherwise }
\end{array}\right.
$$

Where $\mathcal{E}$ is a very small number and for complex systems, it can be set as zero.

5) Best Constant weight

The best constant weighing method is defined as in 5.24:

$$
a_{i j}=\left\{\begin{array}{lr}
\alpha & j \in N_{i} \\
1-\operatorname{deg}_{\text {out }}\left(v_{i}\right) \alpha & i=j \\
0 & \text { otherwise }
\end{array}\right.
$$

In [42], it has been shown that the optimum choice for $\alpha$ in best constant weighing method is $\alpha^{*}=\frac{2}{\lambda_{1}(L)+\lambda_{n-1}(L)}$ where $\lambda \mathrm{i}(L)$ denotes $\mathrm{i}^{\text {th }}$ largest eigenvalue of $\mathrm{L}$ in which $\mathrm{L}$ is the Laplacian matrix.

\subsubsection{ITERATION COMPARISON OF DIFFERENT UPDATING RULES}

Two different cases have been used in this section in order to compare the needed iteration for agreement for each updating matrix.

The first network is assumed to have 20 nodes in its network layer. The maximum number of edges allowed is 40 and the minimum graph connectivity that is required is 0.18 . These requirement is used by the program and a proper network of agents is designed. Then different mentioned updating rules are implemented on the same topology to see how these updating rules can affect the number of iteration needed for reaching an agreement. The graph topology is shown in figure 5.6 and the convergences of various updating methods are demonstrated in figure 5.7. It is obvious that the uniform matrix has a very slow convergence rate, which make it inappropriate for online purposes and between other solutions the Mean Metropolis matrix has the best speed of convergence. It converges in less than 10 iterations for this network. 


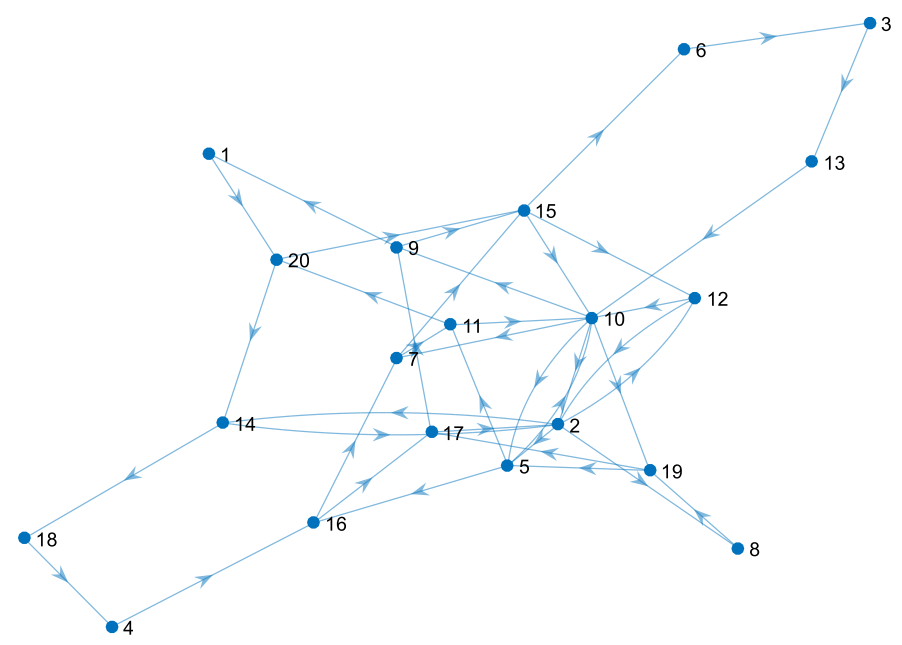

Figure 5.6: Topology of network designed for 20 nodes
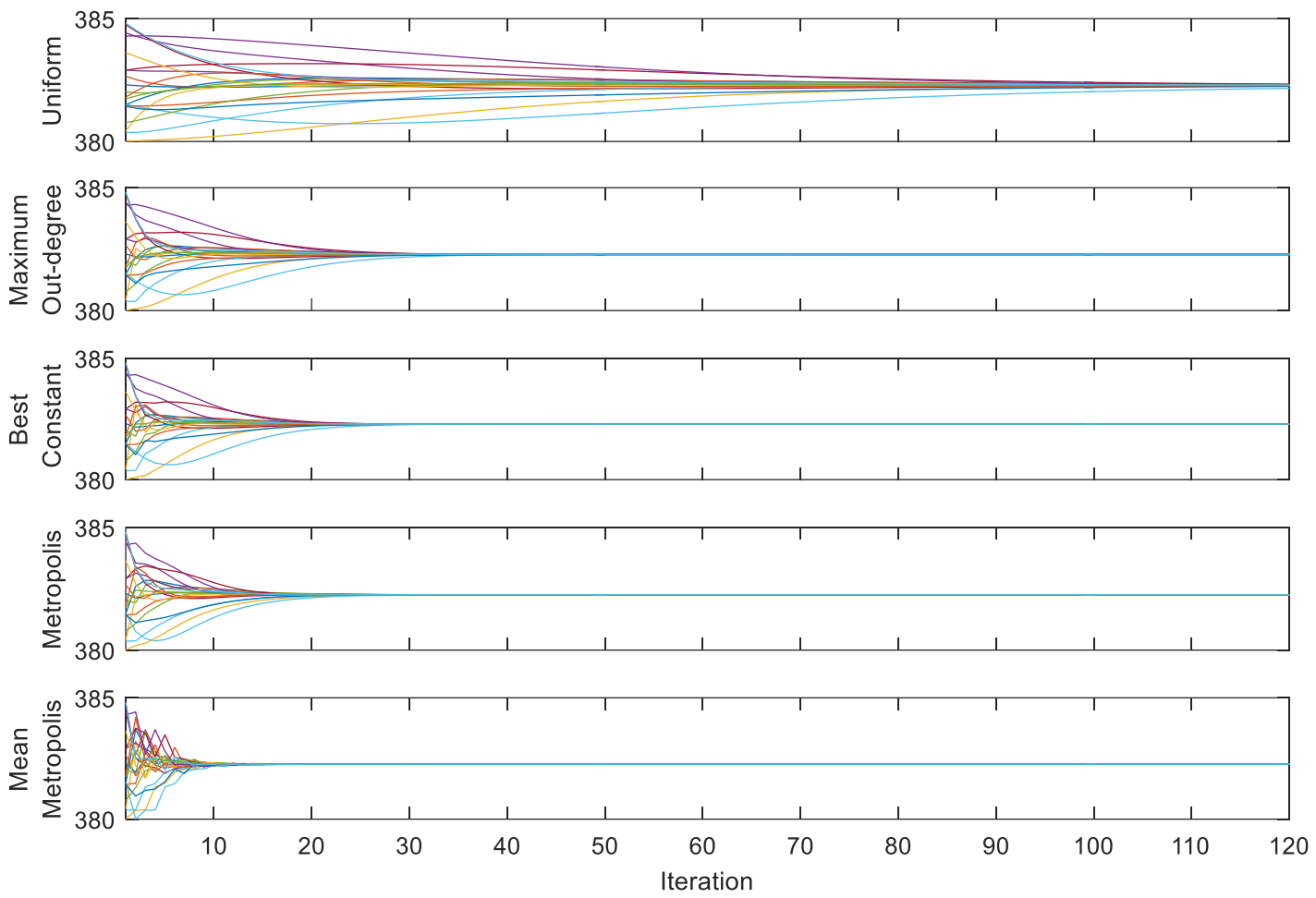

Figure 5.7: The convergence of different updating methods for network of 20 nodes

The second network is assumed to have 100 nodes in its network layer. The maximum number of edges allowed is 300 and the minimum graph connectivity that is required is 0.25 . These requirements are used by the program and a proper network of agents is designed, which is shown in figure 5.8. 


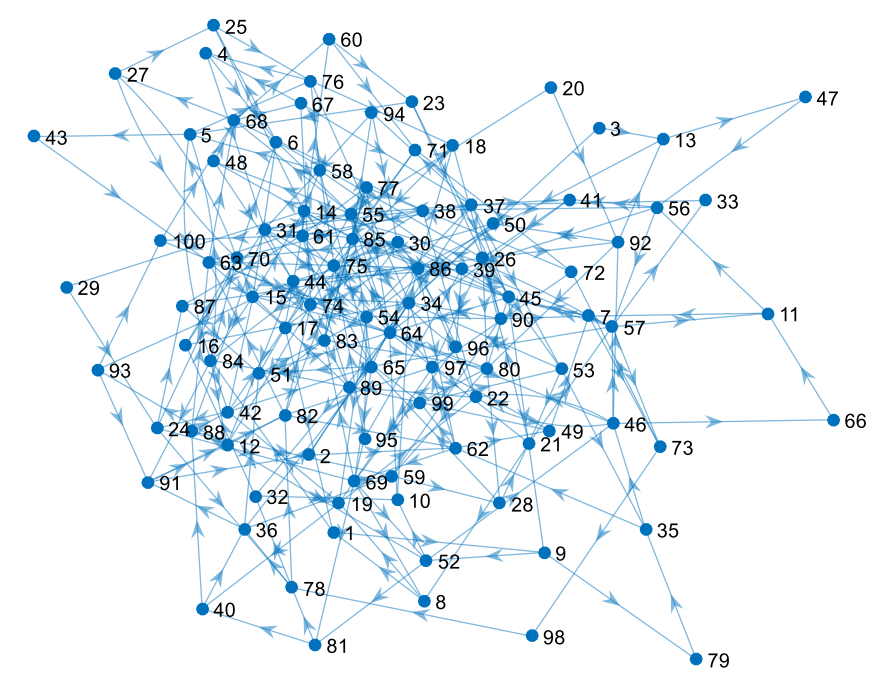

Figure 5.8: Topology of network designed for 100 nodes
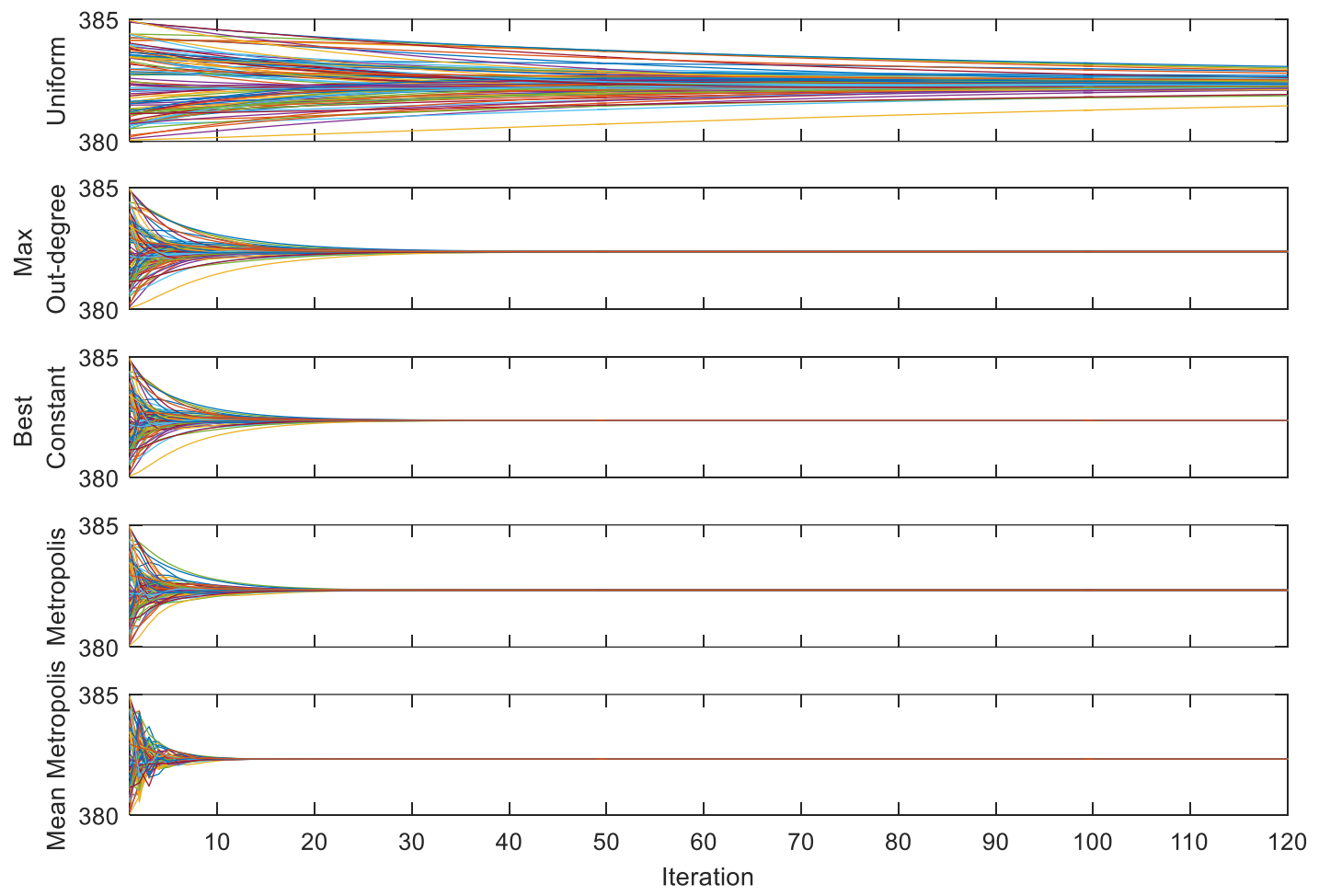

Figure 5.9: The convergence of different updating methods for network of 100 nodes

Then the aforementioned updating rules are implemented on the same topology to investigate the convergence of various updating methods and the results are demonstrated in figure 5.9. Again, it shows that the uniform matrix has a very slow convergence rate and the best solution is Mean Metropolis, which has the best speed of convergence. It converges in less than 10 iteration for this network as well. 
An important conclusion about consensus algorithm is that the agreement time delay is not really dependent on the size of the system; it is mostly affected by the network structure design and the updating matrix chosen for it. This is why that for two different systems with 20 and 100 nodes, by using mean metropolis method, the number of iterations needed for agreement is less than ten iteration. Another positive point of the consensus solution is that the calculation burden is not going to be changed by the size of the whole system. In fact, it only depends on the number of immediate neighbors of each node.

\subsection{TIME DELAYS OF INFORMATION SHARING AND REACHING TO AN AGREEMENT}

\subsubsection{DIFFERENT DELAYS OF THE SYSTEM}

For a fully connected system, the number of connections equals to n(n-1). The system can reach consensus within one step. However, for practical power systems, the iteration matrix is usually a sparse matrix, and so it takes time to reach an agreement. This time interval is called agreement time delay. There are also two other possible time delays, namely the channel's time delay and the controller's time delay. All these three different kinds of delay are shown in figure 5.10.

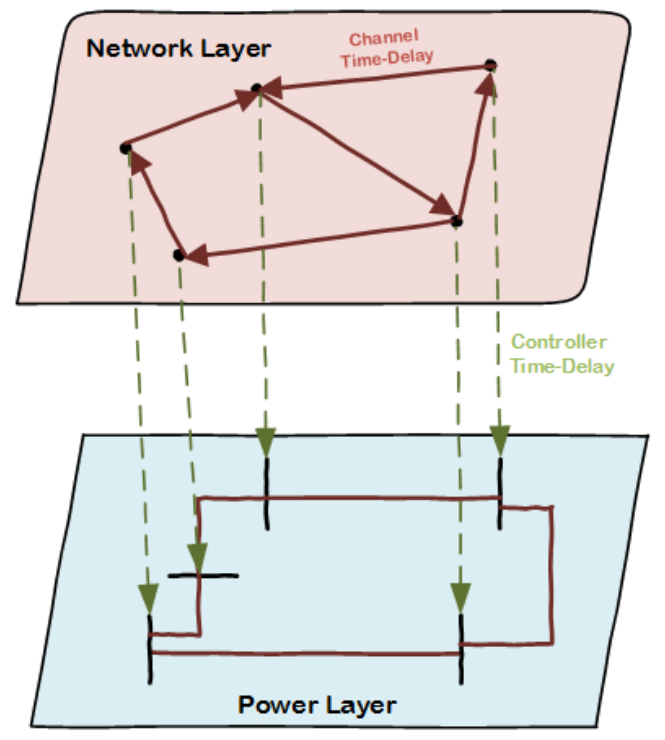

Figure 5.10: Three different time delays of the system

\subsubsection{CHANNEL'S TIME DELAY EFFECT ON CONVERGENCE OF ACA}

As it is been investigated in [38], the upper bound on the admissible channel time delay in the network is inversely proportional to $\lambda_{\mathrm{n}}$, which is the largest eigenvalue of the Laplacian of the information flow. From the Gersgorin theorem, it can be concluded that $\lambda_{n} \leq 2 d_{\max }(G)$ where $d_{\max }(G)$ is the maximum outdegree of all nodes of graph G. Therefore, a sufficient condition for convergence of consensus protocol is 


$$
\tau \leq \frac{\pi}{4 d_{\max }(G)}
$$

This means that networks with nodes having relatively high out-degrees cannot tolerate relatively high communication time delays.

By changing the aforementioned inequality $\left(\lambda_{n} \leq 2 d_{\max }(G)\right)$ to an equation, we would have:

$$
2 d_{\max }(G)=k \lambda_{n}
$$

Where for $k>0$ equation 5.26 will satisfy the mentioned inequality. By substituting the equation 5.26 in 5.25 , we will have an equality described in 5.27 where scaling down the weights of a digraph an arbitrary large time-delay can be tolerated. There is a trade-off here in which the negotiation speed or $\lambda_{2}$ degrades by a factor of $1 / k>0$ so that the delay comes in a tolerable range. In another words, there is a trade-off between the robustness of protocol to the channel's time delay and its performance. [38]

$$
\tau \leq \frac{\pi}{2 k \lambda_{n}}
$$

\subsubsection{AGREEMENT TIME-DELAY AND NEGOTIATION SPEED}

In order to find out how much time it takes for the consensus block to provide the average value for the controller, we need to first know how many iterations will be performed to achieve an agreement. After that, the total time interval needed by the consensus block to provide the result can be calculated.

\section{1) Negotiation Speed (needed iteration for reaching an agreement)}

As discussed previously, the speed of the consensus-based information discovery algorithm is directly determined by the Laplacian matrix of the graph and its second largest eigenvalues (Fiedler eigenvalue). Since it takes too long to reach the exact equilibrium, it is necessary to define a terminating criterion [41]. For this purpose, the error at iteration $k$ can be defined as $E_{k}$. If $E_{k}$ satisfies a predefined precision requirement, it can be concluded that a consensus has been reached and the number of steps needed for convergence is approximately determined according to 5.28. E in this equation is the error tolerance and $\lambda_{2}$ is the second largest eigenvalue of $\mathrm{L}$.

$$
K=\frac{-1}{\log _{E}\left(\frac{1}{\left|\lambda_{2}\right|}\right)}
$$




\section{2) Agreement Time Delay}

After calculating the number of iteration needed for the agreement, it is time to calculate the total time delay to make sure that this whole process is acceptable and practical for the microgrid.

In the previous section, it was demonstrated that different updating methods of ACA can be used for information sharing. So, the corresponding updating matrix would be set according to the related equation and the information sharing processes can be shown in 5.1-5.3 accordingly. However, since the Mean Metropolis method can give us the lowest number of iteration for protocol convergence because of the highest Fiedler eigenvalue, it has the coefficients of updating matrix designs based on this method. The second largest eigenvalues of the corresponding updating matrices for the 20-nodes and 100-nodes systems are 0.8111 and 0.9229 respectively. According to 5.28, the estimated required iteration needed for achieving consensus with a tolerance of 0.1 are 11 and 18 respectively, which is supported by Figs. 5.7 and 5.9.

According to 5.28, it can be seen that the speed of information discovery process is not decided by the size of the system but decided by how the buses are connected and how the coefficients are decided. However, it is good to notice that 5.28 is not precisely accurate because some assumptions had to be made during analysis. It is also important to consider that converging speed of the information discovery algorithm is independent of the initial values. This property can also be observed from 5.28. For verification, the local voltage of each agent is set to a random value during the demonstration of different updating methods in section 5.3.2.

The time delay is the key issue for online voltage control of a microgrid. Generally, the time delay consists of the communication and calculation time delays. In this case, the calculation time delay in MATLAB can be ignored, and the communication time delay depends on the number of iteration needed for agreement and the length of each iteration. The length of each iteration itself depends on the communication speeds in terms of megabit/seconds so the communication time delay can consequently be achieved according to equation 5.29.

$$
\begin{aligned}
& D_{\text {tot }}=D_{\text {cal }}+D_{\text {com }} \\
& D_{\text {com }}=\frac{n_{i t} \times n_{e} \times n_{b}}{V}
\end{aligned}
$$

Where $D_{t o t}$ is the total time delay related to the information sharing process in each agreement process. $D_{c a l}$ is the calculation time delay that is ignored here and $D_{c o m}$ is the communication (or channel) time delay. $n_{i t}$ is the number of iteration to reach the required termination error; $n_{e}$ is the number of element in an information sharing matrix and $n_{b}\left(n_{b i t} \times n_{p}\right.$ [19]) is the number of bits used to represent the element in an information sharing matrix. Finally, $V$ is the data communication speed in terms of bits/second [19]. So, by setting $n_{e}=4, n_{b i t}=16, n_{p}=8, V=8 \mathrm{Mb} / \mathrm{s}$ as practical numbers [19], for the system with 20 nodes which achieves consensus in 11 iterations, the time delay is equal to $0.000704 \mathrm{sec}$ and for the 100 node-system 
that reaches the consensus agreement in 18 iterations this time delay is equal to $0.001152 \mathrm{sec}$. By increasing the communication speed to $20 \mathrm{Mb} / \mathrm{sec}$, these delays will be reduced to 0.000281 and $0.00046 \mathrm{sec}$. It is shown in section 5.5.2 that a delay less than 1 millisecond is almost negligible and does not have any effect on the performance of the average voltage regulator of the power system.

\subsubsection{CONTROLLER TIME-DELAY AND THE POSSIBLE THRESHOLD FOR UPDATING THE CONTROLLER}

After calculating the agreement time delay based on the communication's speed of the network, it is important to consider the controller time delay. The whole microgrid has a threshold for delay. So it should be noted that the total time delay of agreement and controller should not exceed this threshold.

\subsection{AVERAGE VOLTAGE REgUlation}

In order to investigate the average voltage controller, the nonlinear modeling of the system shown in figure 5.11 is simulated in MATLAB software. It is been assumed that a mostly reactive load is added to the system at bus 1 at 1.5 seconds and it has been removed at 2 seconds. So, there is a reactive load increase in the system for 0.5 seconds causing an average voltage drop, and the controller performance is analyzed for this situation.

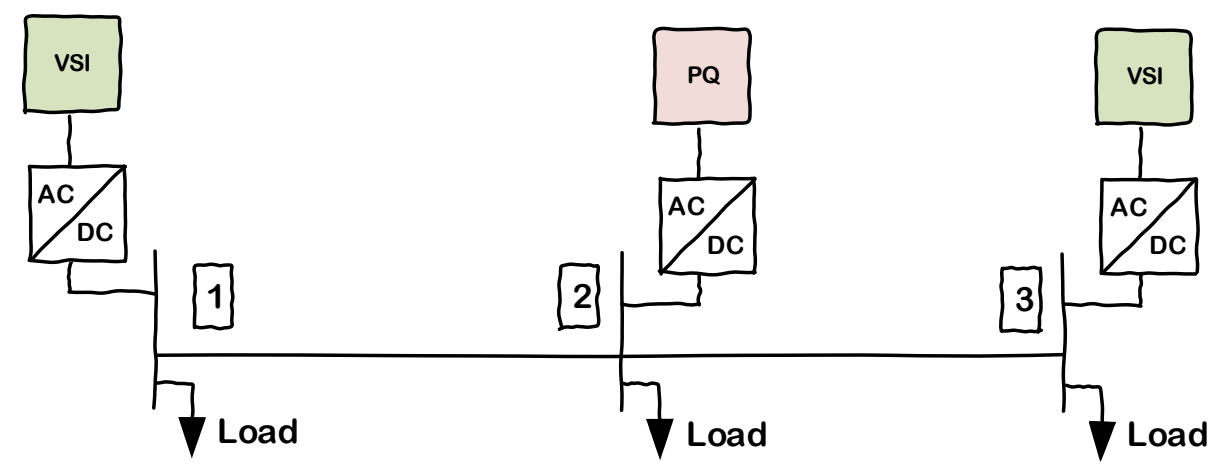

Figure 5.11: Three bus microgrid single line diagram

Figure 5.12 describes the control section that exists for each VSI inverter. They have the inner control loop working in high-frequency bandwidth to track the command voltage and current. Then the primary control, which is slower than inner control, helps to avoid voltage instability by implementing artificial droop characteristics, and finally, at the secondary control section, the voltage and frequency can be regulated to the proper point separately. 


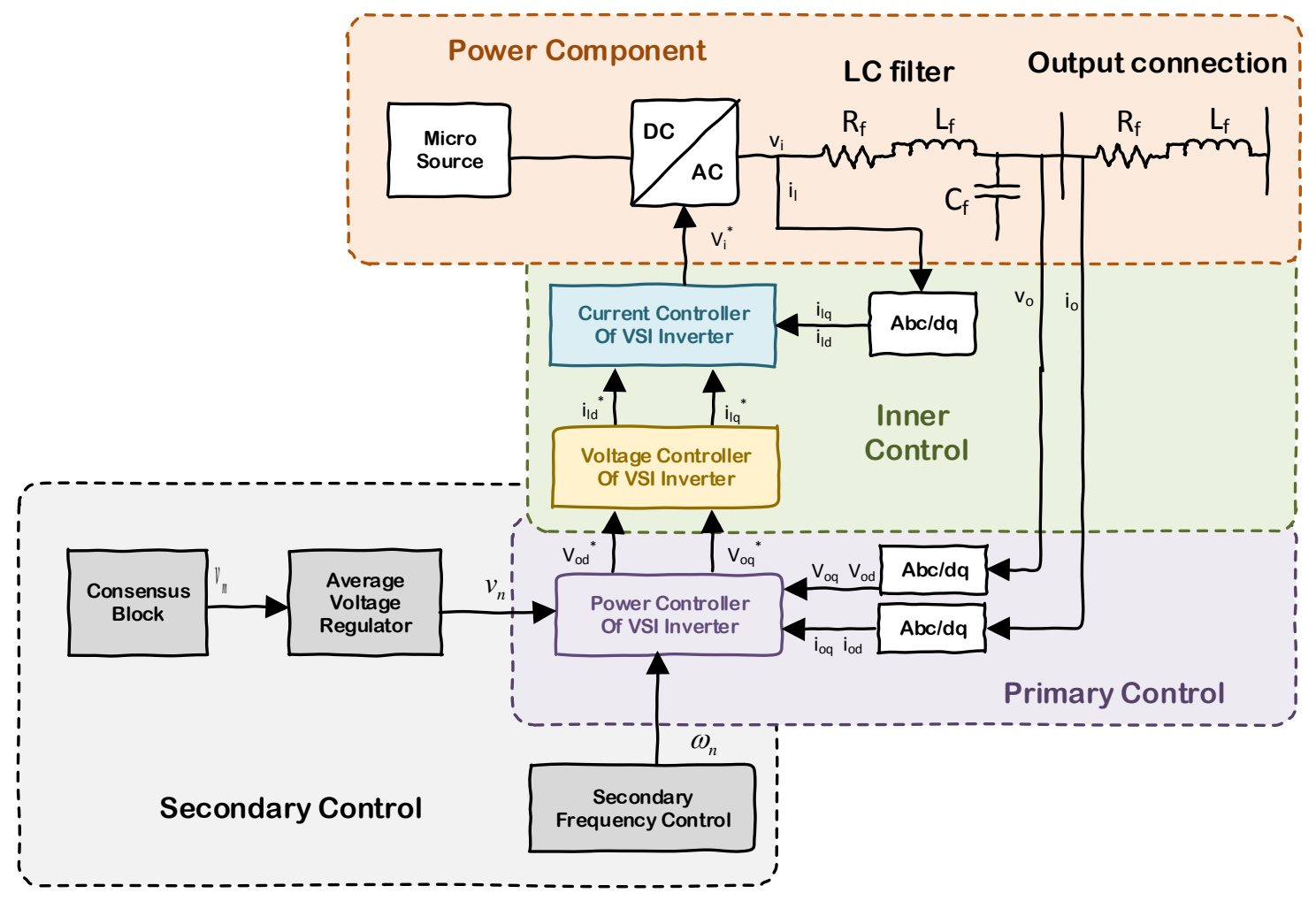

Figure 5.12: Primary and secondary control sections of one VSI inverter

\subsubsection{AVERAGE VOLTAGE REGULATION CONSIDERING IDEAL AGREEMENT (NO DELAY)}

At the remaining part, the effect of having average consensus protocol is compared to the situation in which the controller is not activated. Figure 5.13a shows the voltage at inverters terminal when the average voltage controller uses an average consensus algorithm and when it is deactivated. In figure $5.13 \mathrm{~b}$, the average voltage controller is not activated; So, the voltage of the VSI terminal drops a bit due to the droop gain of the power controller. However, the voltage drops drastically at the terminal of the PQ inverter. Now, comparing this situation to the case that we have the average voltage controller by use of ACA, the voltage drop at PQ inverter terminal is avoided by an increase of the VSI inverter's terminal voltage. So, considering all the voltages together, the average remains the same at the according buses.

Figure 5.14 shows the voltage at bus 1, 2 and 3 in the microgrid. By the use of ACA, the average voltage controller is able to bring back the average of voltages to the predetermined value. The average voltage drop is caused by the reactive load connected at 1.5 seconds and disconnected at 2 seconds to bus 1 . This can be observed by comparing figure 5.14a and 5.14b. After that, figure 5.15 and 5.16 show the produced active and reactive power by inverters during the load change. The dotted lines show the case withoutis no average voltage controller and the solid lines show the case with activated average voltage controller. 

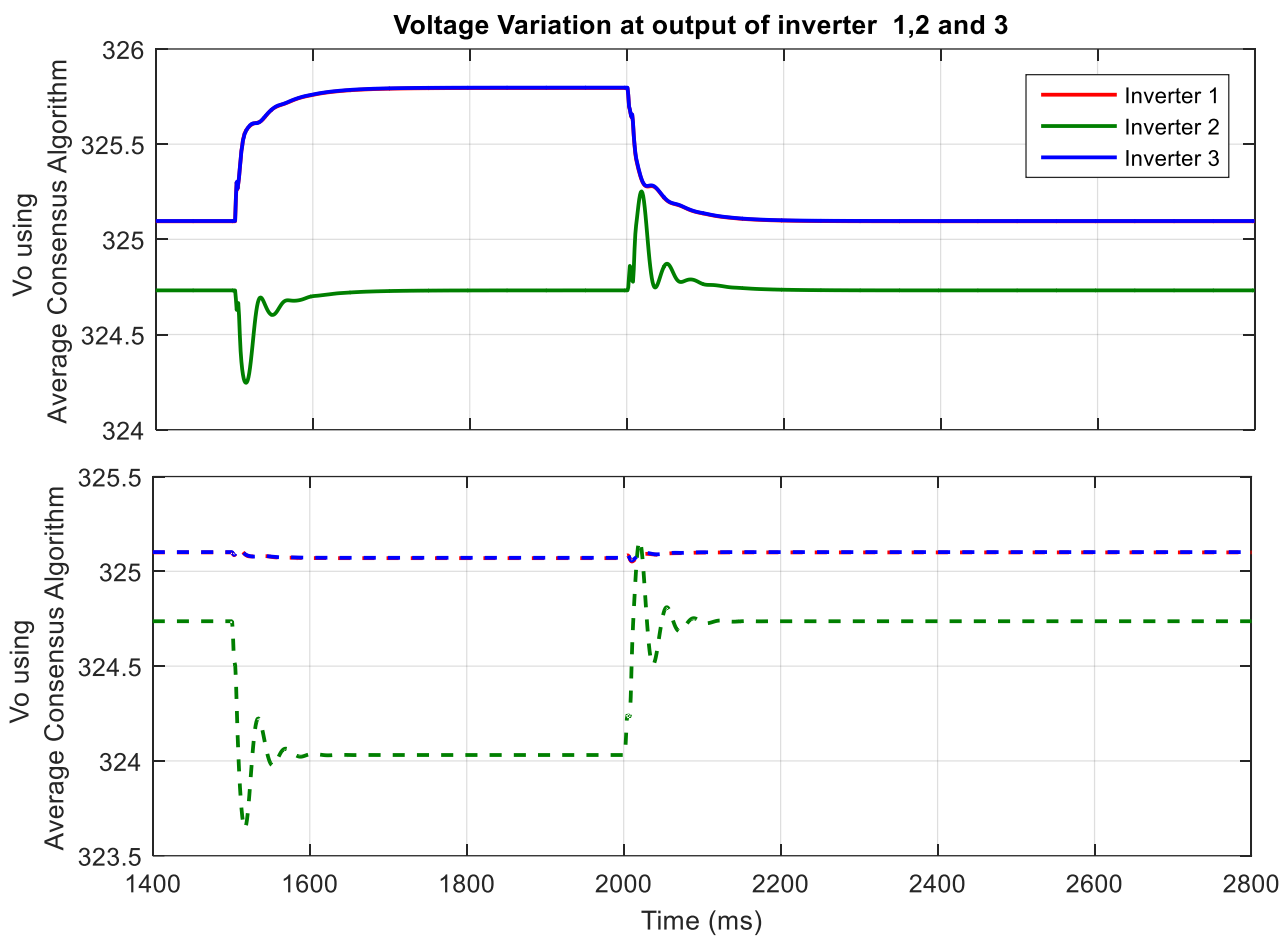

Figure 5.13: Voltages at inverters terminal with (a) and without (b) using the consensus-based average voltage controller
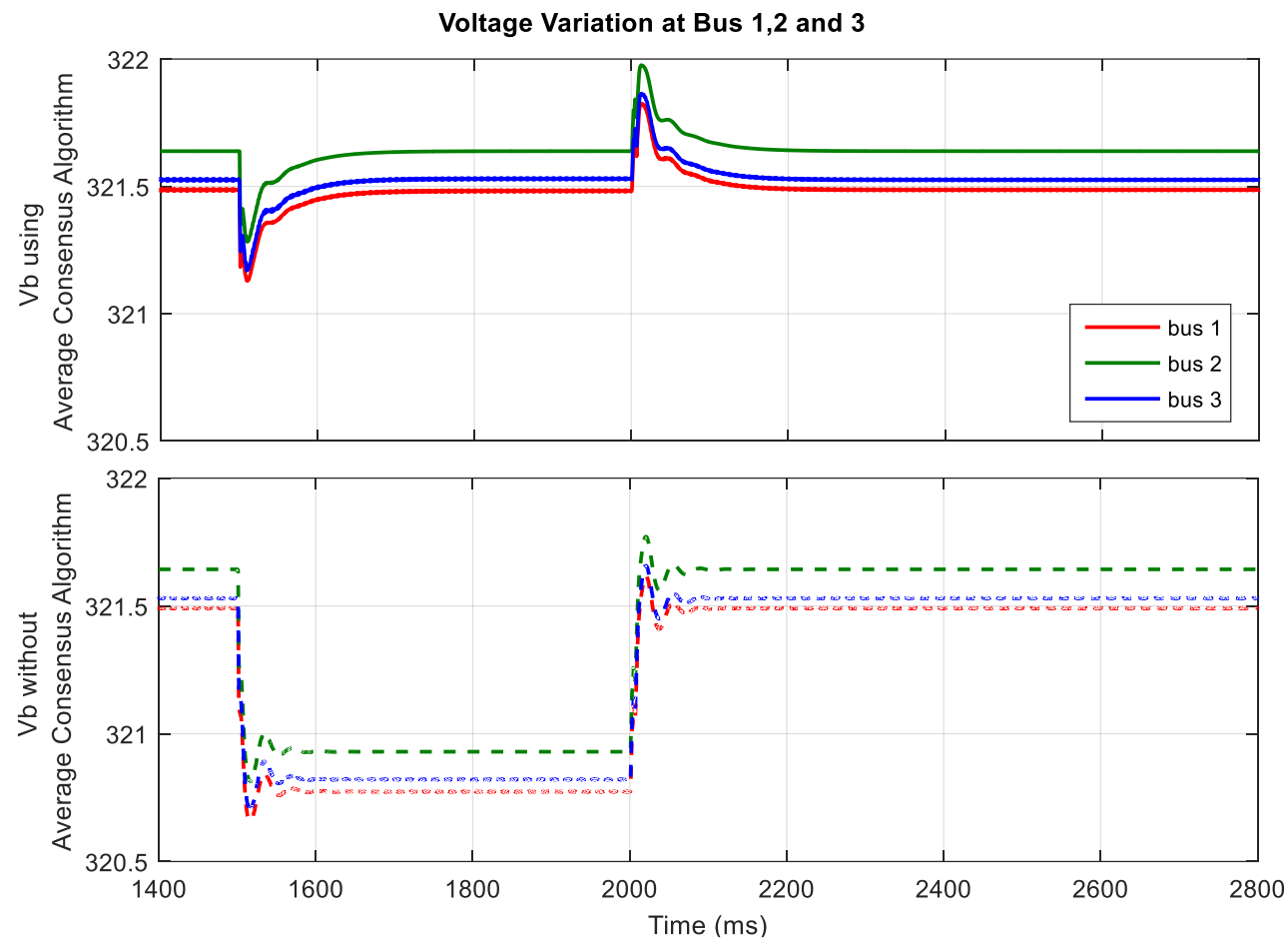

Figure 5.14: Voltages of buses with (a) and without (b) using the consensus-based average voltage controller 
Reactive power produced by inverters at bus 1,2 and 3
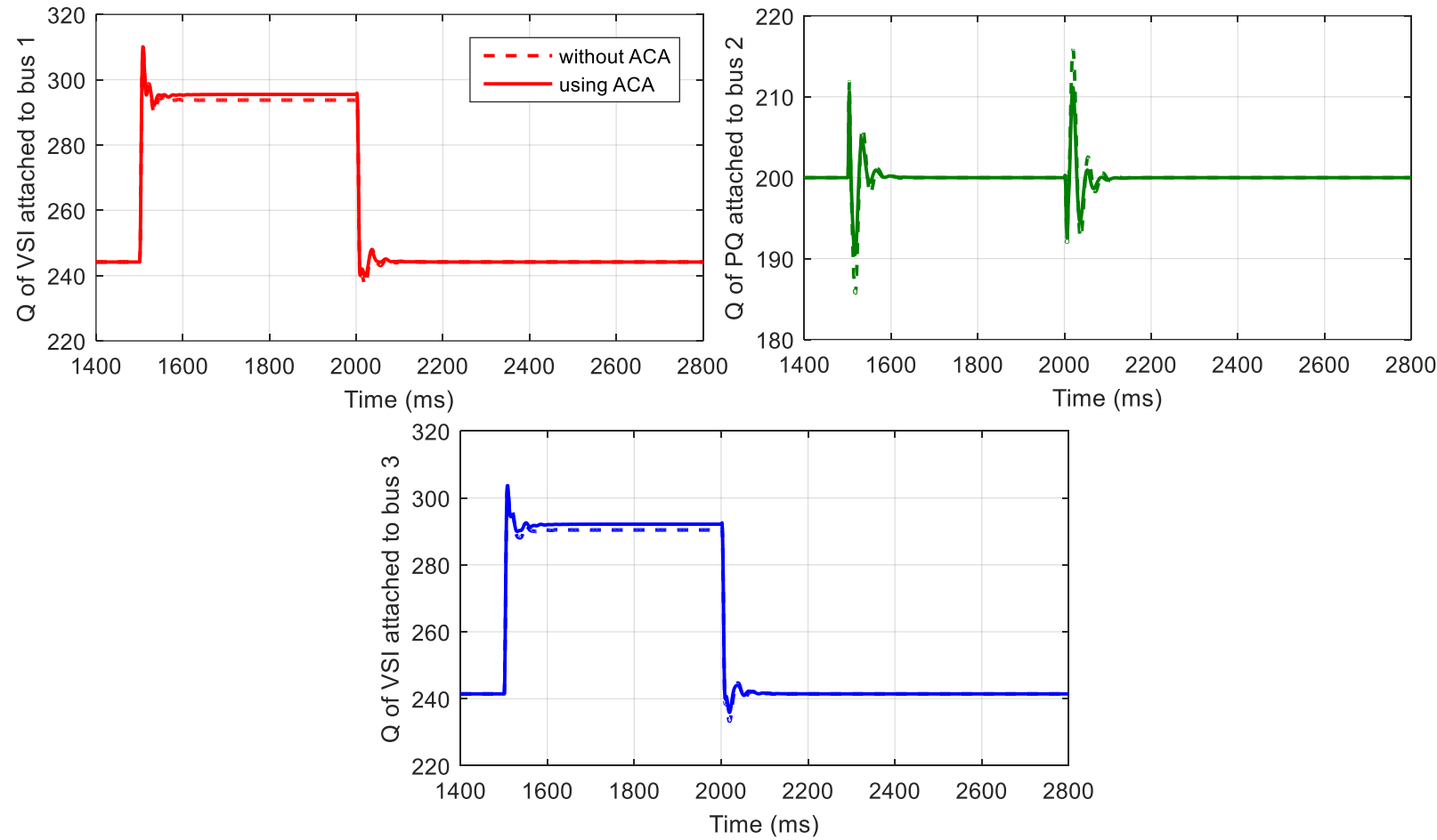

Figure 5.15: Active power produced by VSI and PQ inverters with and without using the consensus-based average

voltage controller (solid lines are representing the ACA based solution and dotted line is without ACA)
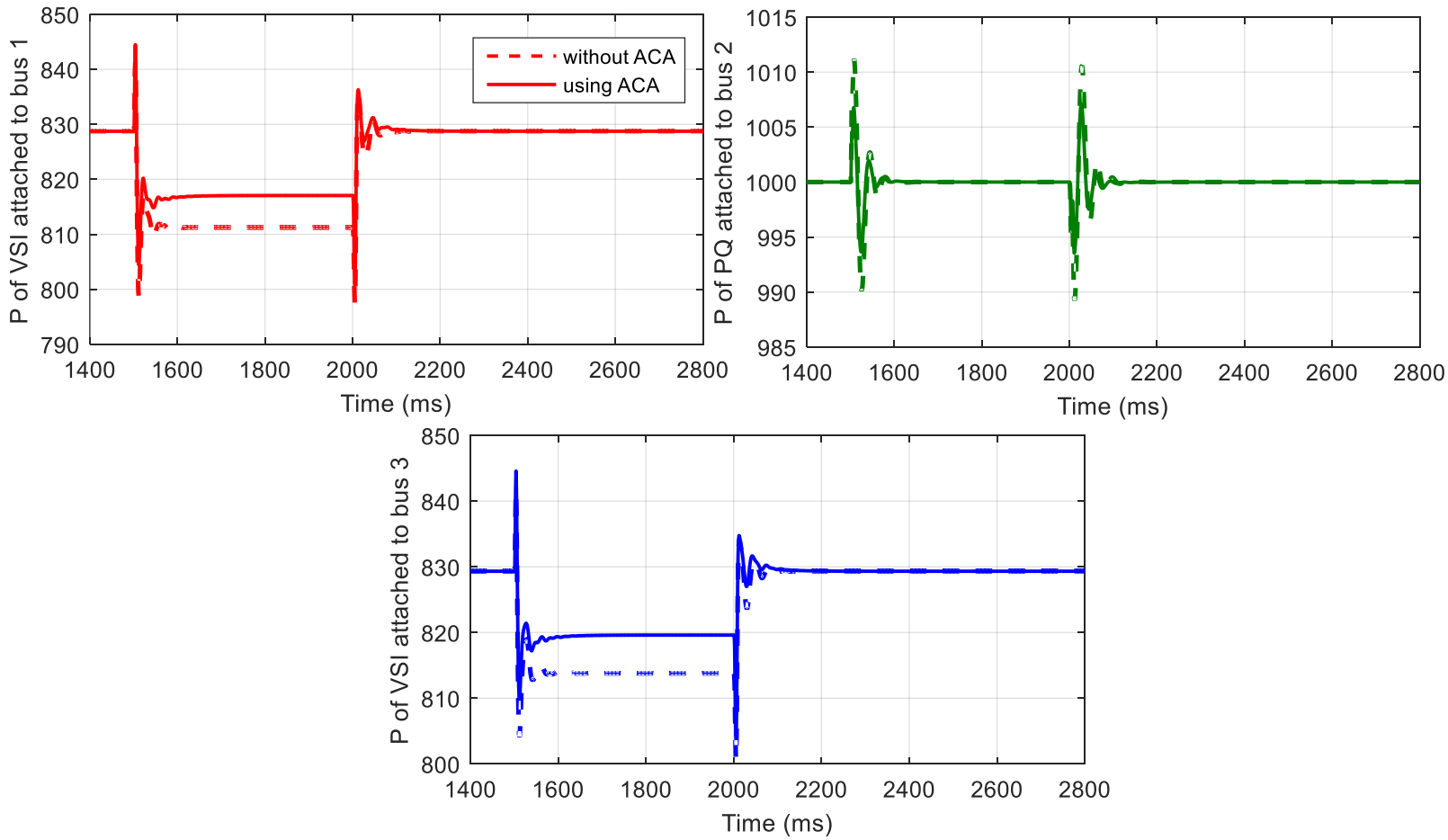
Figure 5.16: Reactive power produced by VSI and PQ inverters with and without using the consensus-based average voltage controller (solid lines are representing the ACA based solution and dotted line is without ACA)

\subsubsection{EFFECT OF CONSENSUS AGREEMENT TIME DELAY ON AVERAGE VOLTAGE REGULATION}

After deciding on the structure of the communication network, the time delay caused by consensus protocol applied to the network of agents can be calculated. This delay is directly related to the number of iteration required to reach an agreement and subsequent awareness between the agents. It is important to know the maximum delay that can be tolerated by the system. Below, the effect of the time delay in controlling the voltage of buses is investigated. In figure 5.17, it is been assumed that all the voltage buses are satisfying the minimum and maximum threshold, and only the average voltage profile needs to be improved and increased by the related controllers located where VSI inverters are.

The error signal passes through a low-pass filter with time constant equal to 0.01 seconds. This filter makes the controller action a bit slower; however, at the same time, it helps the controller to tolerate more delay due to damping of fast and sharp variations.

The time delay is the key issue for online voltage control of a microgrid. Usually, the time delay has the component of communication and calculation time delay. Here, the calculation time delay in MATLAB can be ignored. Figure 5.17 shows the voltage of three buses of the system respectively in different cases formed to analyze the effect of awareness delay on the average voltage regulation. The delay of approximately 1 millisecond does not affect the results in a considerable way, but by increasing the delay, it clearly causes more oscillation, and finally, there would be a threshold after which would make the controller unstable. 


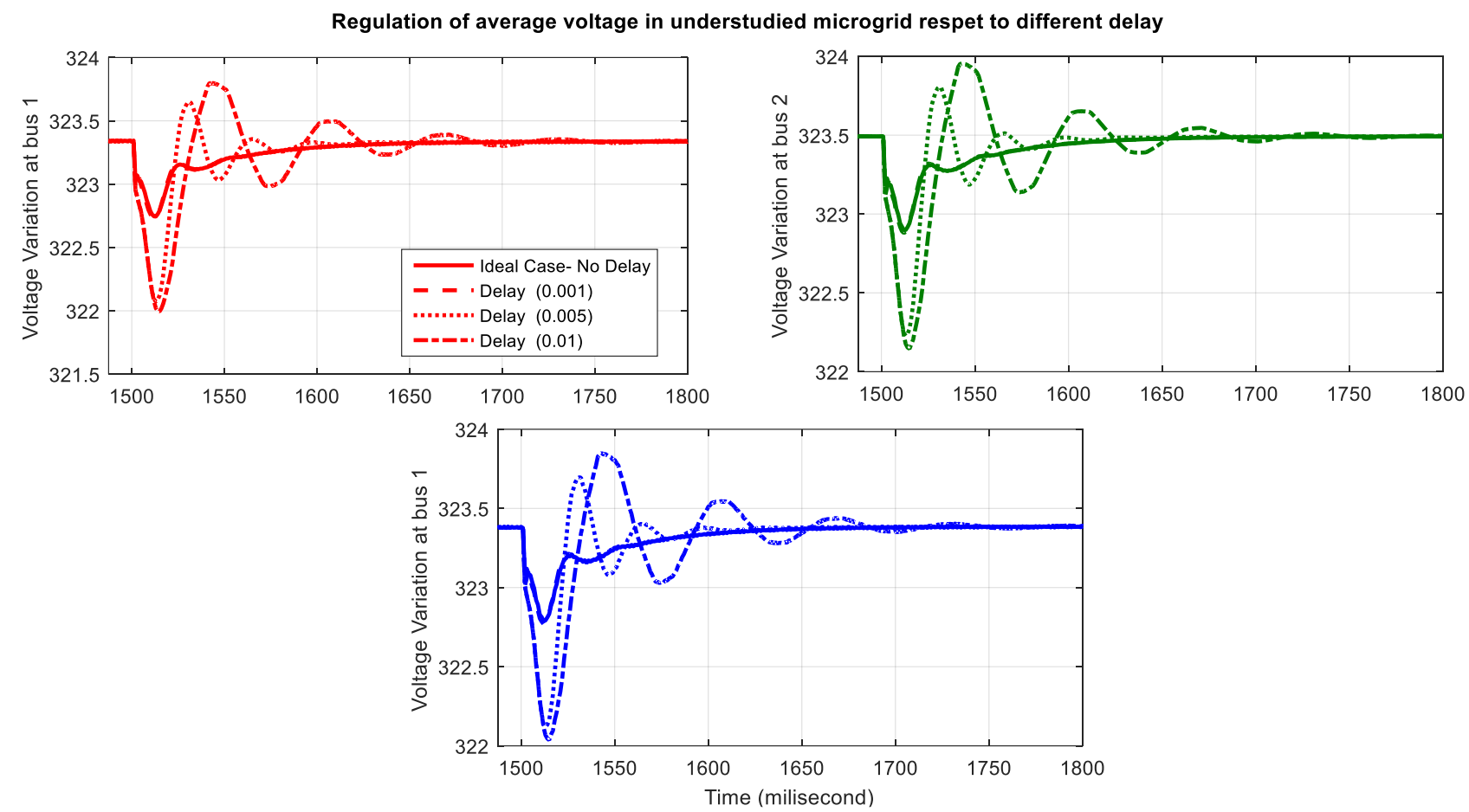

Figure 5.17: Voltages of buses with different agreement time delays using the ACA-based average voltage controller

Then figure 5.18 shows the voltage of all three buses together in one plot. It is been considered that the awareness delay is zero. The picture shows that the average voltage is kept at a predetermined value for the controllers of VSI inverters.

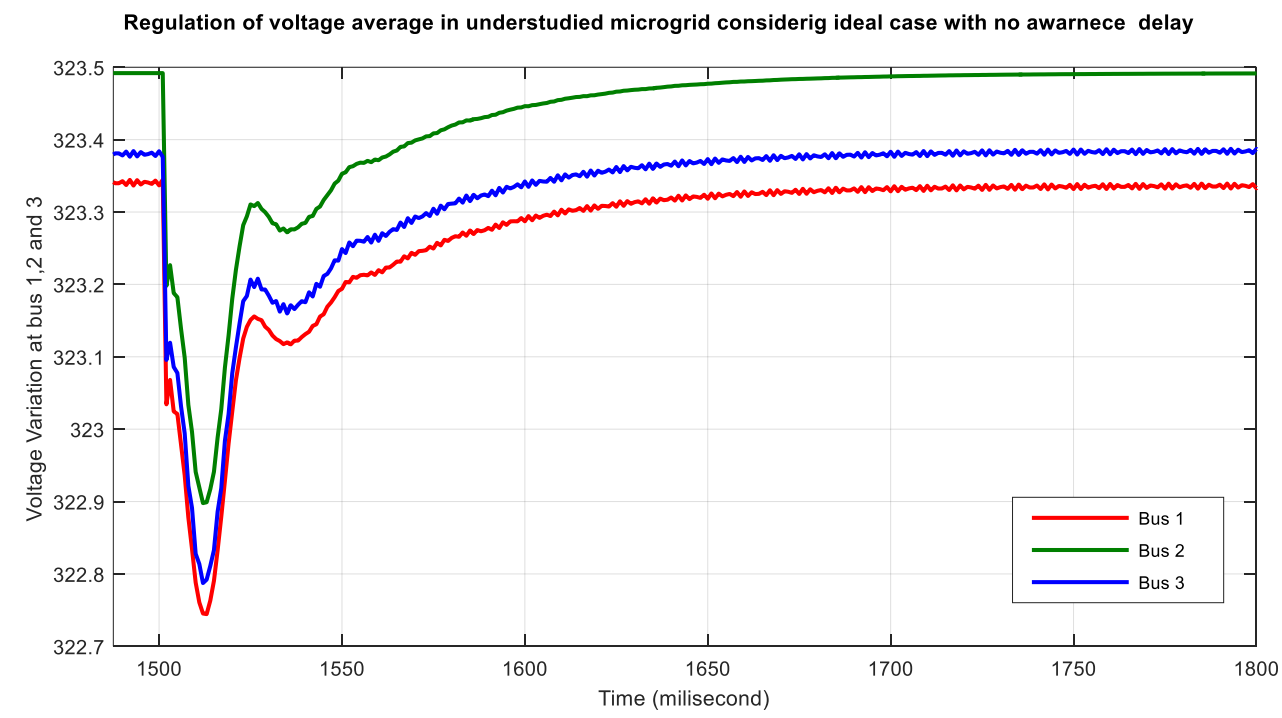

Figure 5.18: Voltages of buses plotted on the same graph using consensus-based average voltage controller 


\subsection{ZONE DEDICATION TO PQ INVERTERS}

The consensus-based voltage control introduced in section 5.5, involved the VSI inverters and there was not any contribution from the PQ inverter's side. In fact, they provided a constant amount of active and reactive power no matter what happened in the system. Here, in the remaining part of this chapter, PQ inverters will also be involved in fixing the voltage profile by dedicating zones to them. Different zones will be defined in a way that includes one of the inverters inside each one. After dedicating a zone to an inverter (whether it is PQ or VSI), that inverter would be responsible for load changes inside the defined zone.

Figure 5.19 shows the understudied 13 bus microgrid consisting of four inverters; two of them are VSI inverters and the other two are PQ inverters. The zone definition is based on the sensitivity of the bus voltage to the power produced by each inverter, assuming that there is one unit of load change in that specific bus. Figure 5.20 shows the zone's definition in the understudied microgrid. 


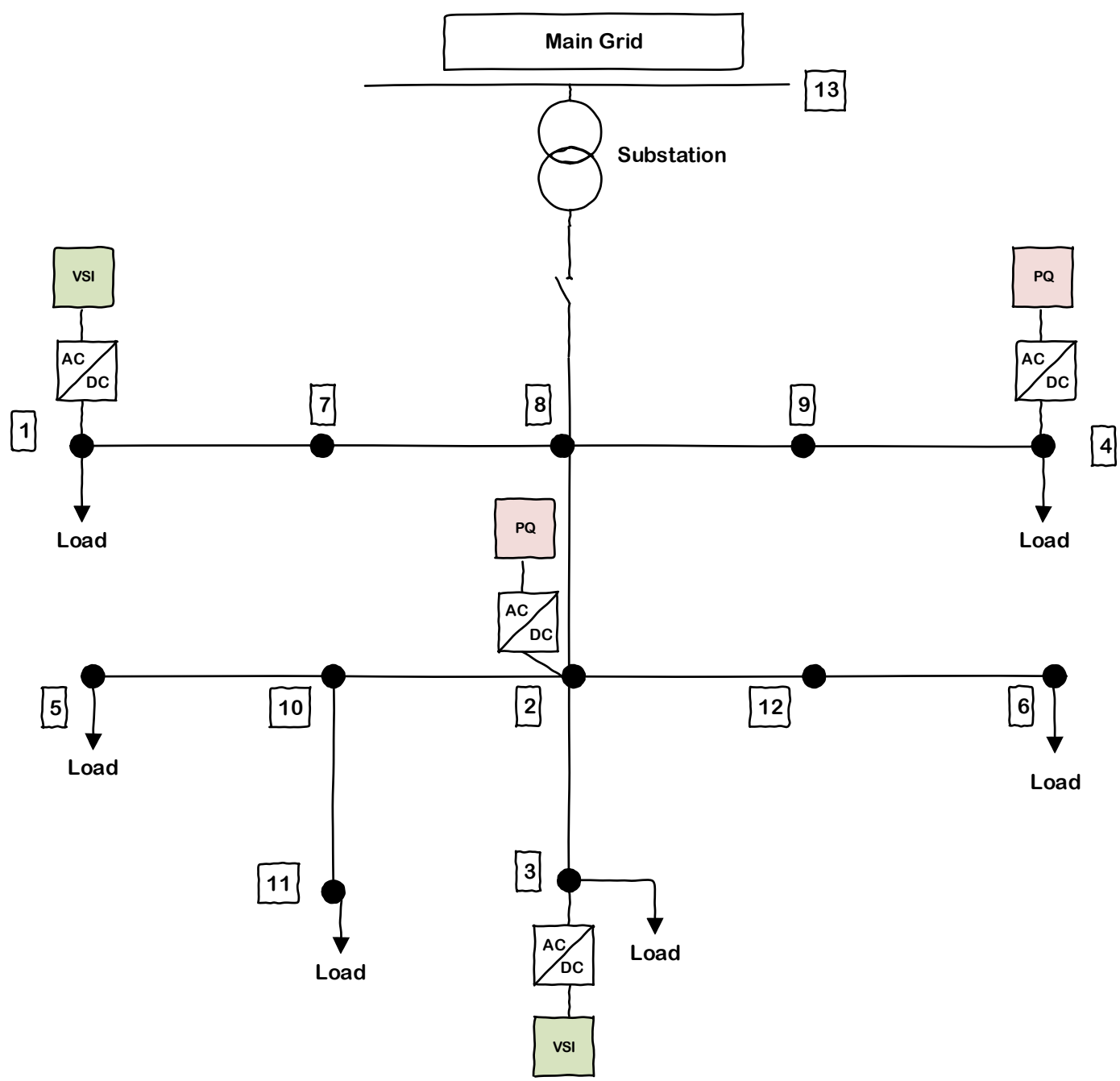

Figure 5.19: Understudied 13 bus microgrid with two PQ inverters and two VSI inverters

As discussed earlier, there are four inverters attached to this microgrid so there would be 4 zones inside the microgrid (since each load is more sensitive to one of the inverters, the number of zones would be equal to the number of inverters). Figure 5.20 shows how these zones include different buses in the system to improve the microgrid's voltage quality. These zone dedications are defined based on experimental results, however, the sensitivity analysis for coming up with the zones definitions in a systematic way is discussed later around in 5.6.1. When a zone includes a specific bus inside, it means it better supports the added load to that bus by the corresponding inverter for having a smoother voltage profile.

The zone dedication approach will help improve the voltage profile by reducing the variance of nodes voltages from the desired average value. In fact, the average voltage of all nodes would be equal to the desired average value by the use of the mentioned consensus algorithm among VSI inverters. However, the 
variance of bus voltages from the average value may not be equal in different situations - and, in some cases, they even deteriorate the minimum and maximum thresholds of the voltage profile. .

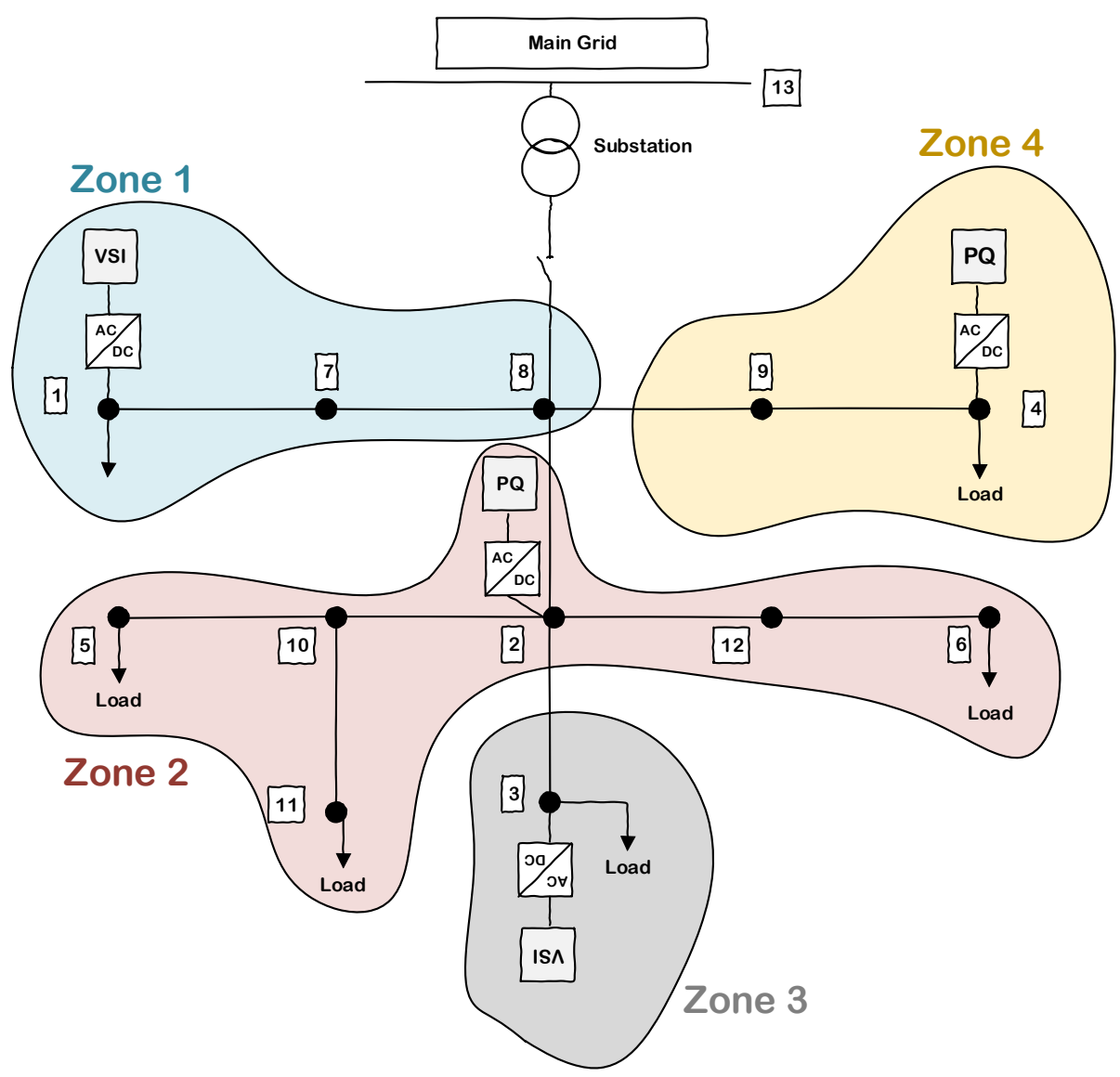

Figure 5.20: Zone dedication to $P Q$ and VSI inverters of the Understudied 13 bus microgrid considering the bus voltage sensitivity to the power production of inverters

The implemented droop-based primary and consensus-based average voltage control in secondary of VSI inverters make them responsible for all the load changes in the system no matter where it is located. They all participate to supply the demanded active and reactive power and keep the average voltage at a desired level. This control is necessary and inevitable because it helps to avoid voltage and frequency instability. In other words, each islanded microgrid needs to have at least one inverter working at VSI mode and working in this mode means that they do not decide how much power they are producing; instead, they have to produce power until the voltage and frequency reach the predefined states. So, in order to implement the zoning scheme above, only PQ inverters have to take actions when they see a demand in their zone. It is good to notice that each PQ inverter will understand the power demand inside its territory by measuring the power flow of tie lines, which connect it to other zones. 
Based on the discussion above, it is clear that the VSI inverters do not need to measure their tie lines flow and if only PQ inverters do their job regarding the supply of the demanded load in their area, it means the VSI inverters automatically supply the rest of system that is their own areas. This fact is shown in figure 5.21 by removing the VSI zone definition from the picture. Also, the tie line of each zone in the microgrid is shown with the same color as the zone. Zone 4 has only one tie line to consider while zone 2 has 2 tie lines.

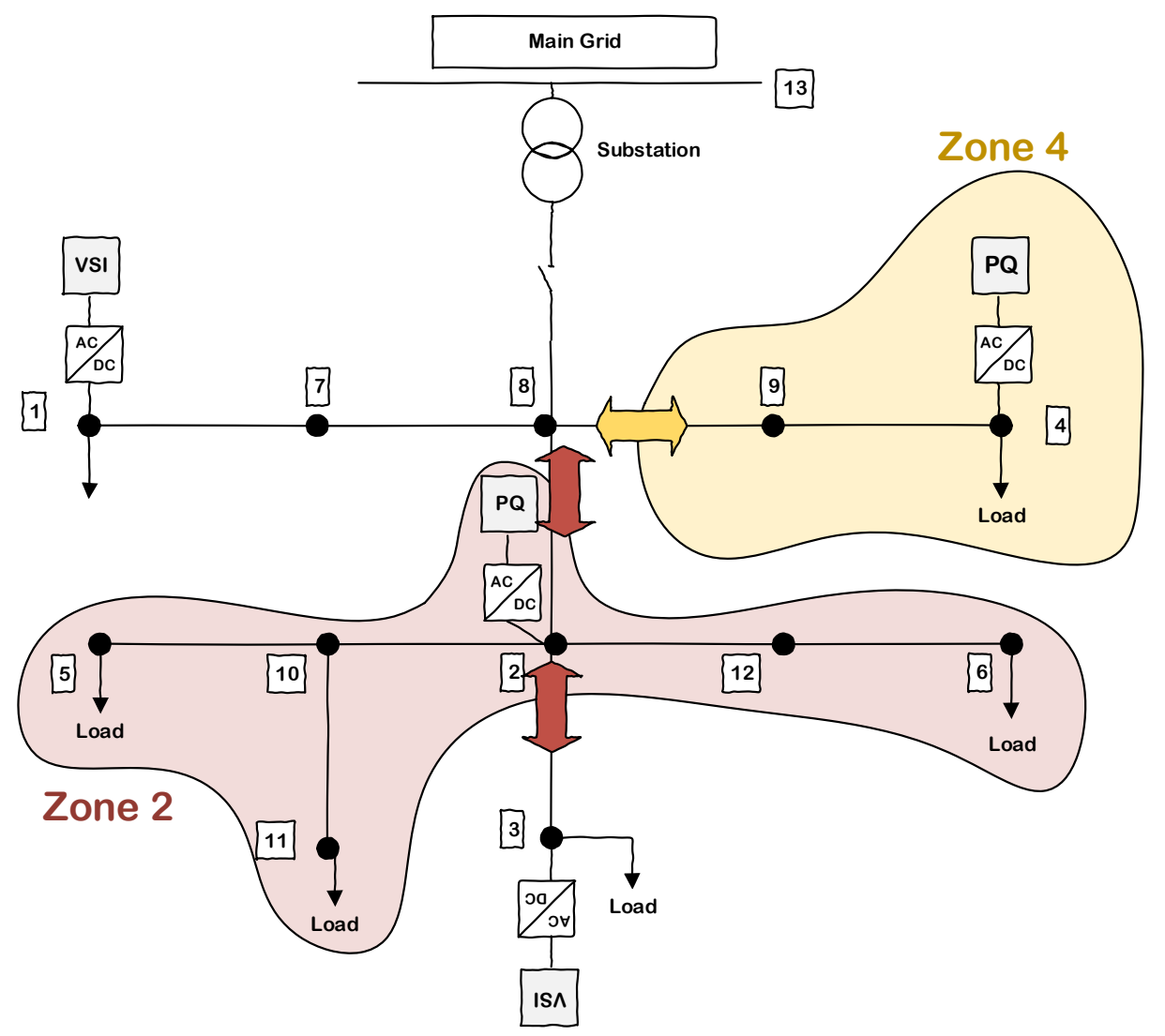

Figure 5.21: Remained zones related to PQ inverters of the Understudied 13 bus microgrid along with their tie lines

\subsubsection{REQUIRED SENSITIVITY ANALYSIS FOR ZONE DEFINITIONS}

In this section the voltage sensitivity in microgrid is analyzed to achieve better voltage regulation in case of any changes in the load demand. Two methods are introduced and discussed in this dissertation.

One of the most common method in the literature such as [43] is the Jacobian Based method. In this method, first the load flow is solved to find out the values of $\mathrm{P}$ and $\mathrm{Q}$. Then the inverse of Jacobian matrix at that operating point is used to find the sensitivity of the system. The procedure is as follows: 


$$
\begin{aligned}
& {\left[\begin{array}{l}
\Delta P \\
\Delta Q
\end{array}\right]=\left[\begin{array}{ll}
\frac{\partial P}{\partial \delta} & \frac{\partial P}{\partial V} \\
\frac{\partial Q}{\partial \delta} & \frac{\partial Q}{\partial V}
\end{array}\right] \cdot\left[\begin{array}{c}
\Delta \delta \\
\Delta V
\end{array}\right]} \\
& {\left[\begin{array}{c}
\Delta \delta \\
\Delta V
\end{array}\right]=\left[\begin{array}{ll}
{\left[S_{\delta p}\right]} & {\left[S_{\delta q}\right.} \\
{\left[S_{v p}\right]} & {\left[S_{v q}\right]}
\end{array}\right] \cdot\left[\begin{array}{l}
\Delta P \\
\Delta Q
\end{array}\right]} \\
& \left.\Delta V=S_{v p} \Delta P+S_{v q} \Delta Q\right)
\end{aligned}
$$

Since $S_{v p}$ 's is mostly smaller than $S_{v q}$, the first term could be ignored to use the simpler format which is

$$
\Delta V=S_{v q} \Delta Q
$$

In other words, we could find the effect of changing $\Delta Q$ on $\Delta V$ from the matrix of $S_{v q}$. However, this method is not always working very well since it is dependent on the load flow calculation and operating points as well. In fact, if the Jacobian matrix is ill-conditioned which it is the case in most of distribution systems, then, it is hard to find the solution of power flow by using Newton-Raphson method. That is why here two other methods of finding the sensitivity analysis in distribution system is introduced and used.

\section{1) First Method}

For balanced systems such as microgrids, which also assumed to have smaller $G_{i j}$ than $B_{i j}$, Fast Decoupled Load Flow (FDLF) is chosen as the first method. This method is very quick and also it is not dependent on the operating point; those are two important reasons of using this method. In this method, the Jacobian matrix is only derived once and it is not needed to be calculated for each iteration. We can find the Jacobian Matrix in Fast Decoupled Load Flow as follows: 


$$
\left[\begin{array}{l}
{\left[\begin{array}{l}
\frac{\partial f_{p k}}{\partial \delta_{k}}=\sum_{\substack{i=1 \\
i \neq k}}^{N} V_{k} B_{k i} \\
\frac{\partial f_{p k}}{\partial \delta_{i}}=-V_{k} B_{k}(i \neq k)
\end{array}\right] \quad\left[\begin{array}{l}
\frac{\partial f_{p k}}{\partial V_{k}}=0 \\
\frac{\partial f_{p k}}{\partial V_{i}}=0(i \neq k)
\end{array}\right]} \\
{\left[\begin{array}{l}
\frac{\partial f_{p k}}{\partial V_{k}}=0 \\
\frac{\partial f_{p k}}{\partial \delta_{i}}=0(i \neq k)
\end{array}\right]\left[\begin{array}{l}
\frac{\partial f_{Q k}}{\partial V_{k}}=-2 V_{k} B_{k k}-\sum_{\substack{i=1 \\
i \neq k}}^{N} V_{i} B_{k i} \approx V_{k}\left(-2 B_{k k}-\sum_{\substack{i=1 \\
i \neq k}}^{N} B_{k i}\right) \\
\frac{\partial f_{Q K}}{\partial V_{i}}=-V_{i} B_{k i} \approx-V_{k} B_{k i}(i \neq k)
\end{array}\right]}
\end{array}\right]
$$

As seen, the off-diagonal elements of the Jacobian matrix are zeros based on the mentioned assumptions. The diagonal elements can be rewritten so that two new parameters called $B^{\prime}$ and $B^{\prime \prime}$ are defined as follows:

$$
\begin{aligned}
& B_{k k}^{\prime}=\sum_{\substack{i=1 \\
i \neq k}}^{N} B_{k i} ; B_{k i}^{\prime}=-B_{k i}(i \neq k) \\
& B^{\prime \prime}=-2 B_{k k}-\sum_{\substack{i=1 \\
i \neq k}}^{N} B_{k i} ; B_{k i}^{\prime \prime}=-B_{k i}(i \neq k)
\end{aligned}
$$

Here is the simpler format for Jacobian matrix by using $B^{\prime}$ and $B^{\prime \prime}$ :

$$
\left[\begin{array}{c}
{\left[\begin{array}{c}
\frac{\partial f_{p k}}{\partial \delta_{k}}=\sum_{\substack{i=1 \\
i \neq k}}^{N} V_{k} B_{k i}=V_{k} \sum_{\substack{i=1 \\
i \neq k}}^{N} B_{k i}=V_{k} B_{k k}^{\prime} \\
\frac{\partial f_{p k}}{\partial \delta_{i}}=-V_{k} B_{k i}=V_{k} B_{k i}^{\prime}(i \neq k)
\end{array}\right]} \\
{[0]} \\
{[0]} \\
{\left[\begin{array}{c}
\frac{\partial f_{Q K}}{\partial V_{k}}=V_{k}\left(-2 B_{k k}-\sum_{\substack{i=1 \\
i \neq k}}^{N} B_{k i}\right. \\
\frac{\partial f_{Q k}}{\partial V_{i}}=-V_{k} B_{k i}=V_{k} B_{k i}^{\prime \prime}(i \neq k)
\end{array}\right]=V_{k} B_{k i}^{\prime \prime}}
\end{array}\right]
$$


Now by defining $[V]=\left[\begin{array}{cccc}V_{1} & 0 & 0 & 0 \\ 0 & V_{2} & 0 & 0 \\ 0 & 0 & \ldots & 0 \\ 0 & 0 & 0 & V_{n}\end{array}\right]$, we can write $\frac{\partial f_{Q k}}{\partial V_{i}}=V_{k} B_{k i}^{\prime \prime}$ in a matrix format as follows:

$$
\left[\frac{\partial f_{Q}}{\partial V}\right]=\left[V \cdot B^{\prime \prime}\right]
$$

Therefore, for finding the sensitivity of the voltages of the system nodes with respect to the reactive power, we just need to find the inverse of equation 5.39, which is:

$$
\left[\frac{\partial f_{Q}}{\partial V}\right]^{-1}=\left[V B^{\prime \prime}\right]^{-1}=B^{\prime \prime-1} V^{-1}
$$

In the following, the procedure is explained for the understudying microgrid in details. At first, it is been assumed that the bus1 of DG1 is the slack bus so its row and column are eliminated from the $\mathrm{Y}_{\text {bus }}$ matrix to form the B". The result of zone definition based on this consideration is shown in table 5.5.

TABLE 5.5

ZONE CALCULATION CONSIDERING BUS 1 AS SLACK

\begin{tabular}{|l|l|l|l|l|l|l|l|l|l|l|l|}
\hline Bus \# & 2 & 3 & 4 & 5 & 6 & 7 & 8 & 9 & 10 & 11 & 12 \\
\hline Zone & 2 & 3 & 4 & 2 & 2 & 4 & 4 & 4 & 2 & 2 & 2 \\
\hline
\end{tabular}

As it can be seen, there is no bus assigned to zone 1 since bus 1 is assumed to be the slack bus and was eliminated from the $B^{\prime \prime}$. Therefore, we need to repeat this process by assuming another inverter bus except bus 1 to be the slack bus. So, by assuming that the bus 2 is now the slack bus, we can find out which buses are sensitive to the bus 1,3, and 4; but again the buses that are sensitive to bus 2 are not specified.

In other words, the result of sensitivity for each iteration does not reflect the effect of the eliminated bus in the defined zones. Therefore, to solve this problem, we need to repeat this process until all of the inverter buses have been once assigned to be slack bus. Then, we need to take a look at each bus individually to find out the most repeated zone in different cases. For example, for bus 5, zone 2 is repeated the most which shows that it is more sensitive to that DG. The total result is shown in the last row of table 5.6.

TABLE 5.6

ZONE CALCULATION CONSIDERING EACH INVERTER Bus AS SLACK ONCE 


\begin{tabular}{|c|l|l|l|l|l|l|l|l|l|l|l|l|}
\hline Bus \# & 1 & 2 & 3 & 4 & 5 & 6 & 7 & 8 & 9 & 10 & 11 & 12 \\
\hline $\begin{array}{c}\text { Zones } \\
\text { (bus } 1 \text { is eliminated) }\end{array}$ & ----- & 2 & 3 & 4 & 2 & 2 & 4 & 4 & 4 & 2 & 2 & 2 \\
\hline $\begin{array}{c}\text { Zones } \\
\text { (bus } 2 \text { is eliminated) }\end{array}$ & 1 & ----- & 3 & 4 & 1 & 1 & 1 & 1 & 4 & 1 & 1 & 1 \\
\hline $\begin{array}{c}\text { Zones } \\
\text { (bus } 3 \text { is eliminated) }\end{array}$ & 1 & 2 & ----- & 4 & 2 & 2 & 1 & 1 & 4 & 2 & 2 & 2 \\
\hline $\begin{array}{c}\text { Zones } \\
\text { (bus } 4 \text { is eliminated) }\end{array}$ & 1 & 2 & 3 & ----- & 2 & 2 & 1 & 1 & 1 & 2 & 2 & 2 \\
\hline \begin{tabular}{c} 
Accurate Zone \\
\hline
\end{tabular} & 1 & 2 & 3 & 4 & 2 & 2 & 1 & 1 & 4 & 2 & 2 & 2 \\
\hline
\end{tabular}

\section{2) Second Method}

As a second method to find the sensitivity in the electrical system we can easily use the Ohm's Law. By using the equation of $V=Z I$, we will see the effect of increasing in the voltages by increasing the currents of DG's in the network. Therefore, in this method unlike the previous one, we saw the effect of both P and Q simultaneously since we are working with the current which has both $\mathrm{P}$ and Q in it. In this method, after deriving the $\mathrm{Z}$ matrix, by increasing the current of each DG's once at a time, we can see its effect on the voltages of the buses. The one that its voltage has increased the most, relates to the zone of that DG that its current has been increased. In other words, in this method by easily looking at the values of the elements of $\mathrm{z}$ matrix we can find out the corresponding zone. For example, for bus1 we should take a look at the $\mathrm{z}_{11}$, $\mathrm{z}_{12}, \mathrm{z}_{13}$, and $\mathrm{z}_{14}$, which are the corresponding impedances of DGs and can be used for marking the one with the highest impact. In other words, the highest element (the highest impedance) of the first four columns shows that the bus is belonged to which zone.

This procedure can be shown as follows:

$$
\left[\begin{array}{c}
V_{1} \\
V_{2} \\
V_{3} \\
V_{4} \\
\cdots \\
V_{n}
\end{array}\right]=\left[\begin{array}{ccccc}
z_{11} & z_{12} & z_{13} & z_{14} & \cdots \\
z_{21} & z_{22} & z_{23} & z_{24} & \cdots \\
z_{31} & z_{32} & z_{33} & z_{34} & \cdots \\
z_{41} & z_{42} & z_{43} & z_{44} & \cdots \\
\cdots & \cdots & \cdots & \cdots & \cdots \\
z_{n 1} & z_{n 2} & z_{n 3} & z_{n 4} & \cdots
\end{array}\right]\left[\begin{array}{l}
I_{V S I 1} \\
I_{V S I 2} \\
I_{P Q 1} \\
I_{P Q 2} \\
\cdots \\
0
\end{array}\right]
$$




$$
\begin{aligned}
& \Delta V_{1}=Z_{11} \cdot \Delta I_{1} \\
& \Delta V_{1}=Z_{12} \cdot \Delta I_{2} \\
& \Delta V_{1}=Z_{13} \cdot \Delta I_{3} \\
& \Delta V_{1}=Z_{14} \cdot \Delta I_{4}
\end{aligned}
$$

\subsection{SimUlATION AND RESULTS DEMONSTRATION}

The islanded 13 bus microgrid is used to verify the effectiveness of zone dedication in reducing the variance of nodes voltages from the desired average value. Figure 5.21 shows the single line diagram of the microgrid test system along with the zone dedications to PQ inverters. The nominal frequency and voltage are $50 \mathrm{~Hz}$ and $380 \mathrm{~V}$, respectively. The loads are modeled as typical RL loads and the RL branch is also used for the lines between buses. Bus 13 in figure 5.21 shows the point of common coupling. The simulation results are presented in three different scenarios. In the first two cases, the load change is happening in zone 4 ; it is a demand increase for case one and then a demand decrease for case two. In case three, the demand change will happen in zone 2. In all three cases, the average voltage of main buses (PQ buses, VSI buses

and critical load buses including bus 4 and 5) are regulated to the predetermined value of 456 Volt at peak, or 323.4 Volt at rms. It has been assumed that a $2 \%$ mismatch from the average is acceptable. The resulting demonstration will effectively show that by use of average consensus control for VSI inverters, the average voltage is regulated completely but sometimes it is possible that the minimum and maximum requirement is not met by only involving the VSI inverters in voltage control.

\section{1) Case one}

In the first scenario, the islanded microgrid is facing a demand increase in bus 4 located in zone 4 . The line between bus 8 and 9 is the only tie line where its power flow should be considered for this zone. It is assumed that the DG attached to bus 4 is communicating with the agent located at the mentioned tie line to sense the power flow and change its set-point based on that. In this scenario, the active and reactive load increase is equal to $4.5 \mathrm{k}$ watt and $5 \mathrm{k}$ var respectively and it happens at $\mathrm{t}=1.5 \mathrm{sec}$. In order to show the effectiveness of zone dedication of the PQ inverters, the results for both having and not having zones is presented here. 


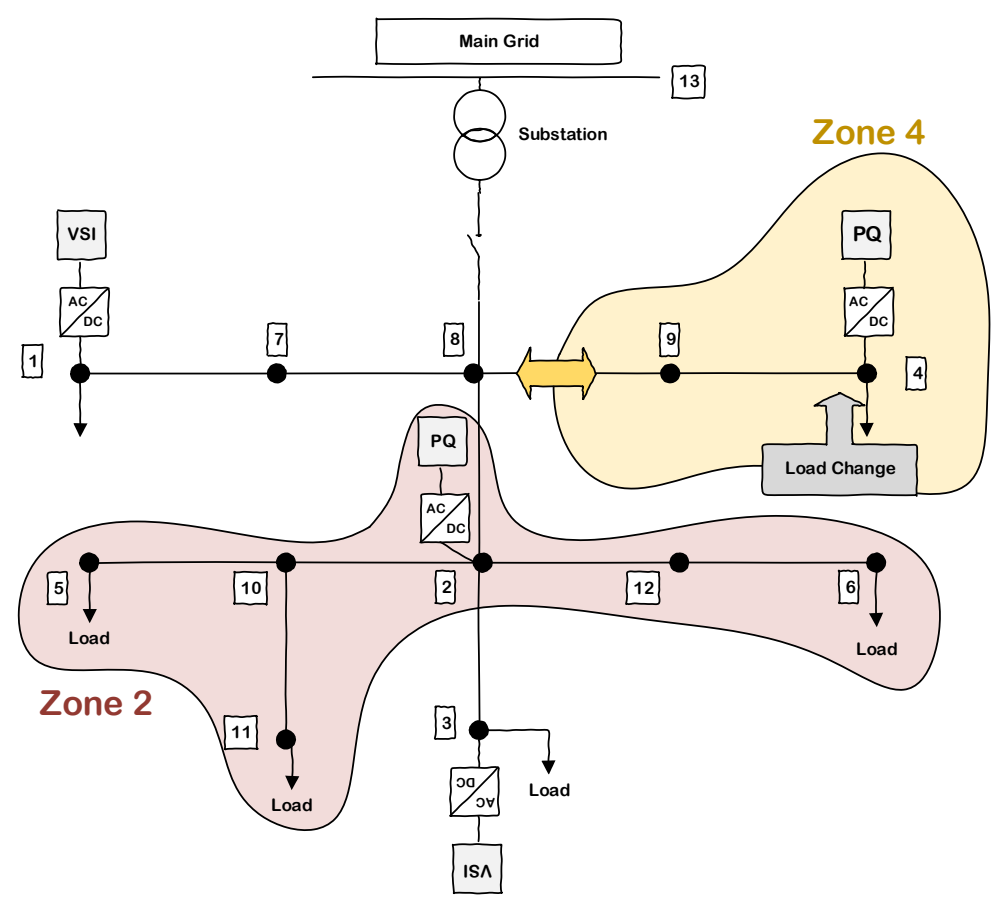

Figure 5.22: Load change location in case one and two

Figure 5.23 shows the main six bus voltages when the traditional voltage control is applied. In traditional voltage control, only the voltages of VSI buses are controlled to be fixed at a specific set-point - for example, $323.4 \mathrm{rms}$. Then, in figure 5.24, the consensus-based average voltage control is implemented through the VSI inverter. In this situation, PQ inverters are not involved yet. Then, by dedicating zones to them, they would have a contribution in voltage control and the result of this involvement is shown in figure 5.25 . 


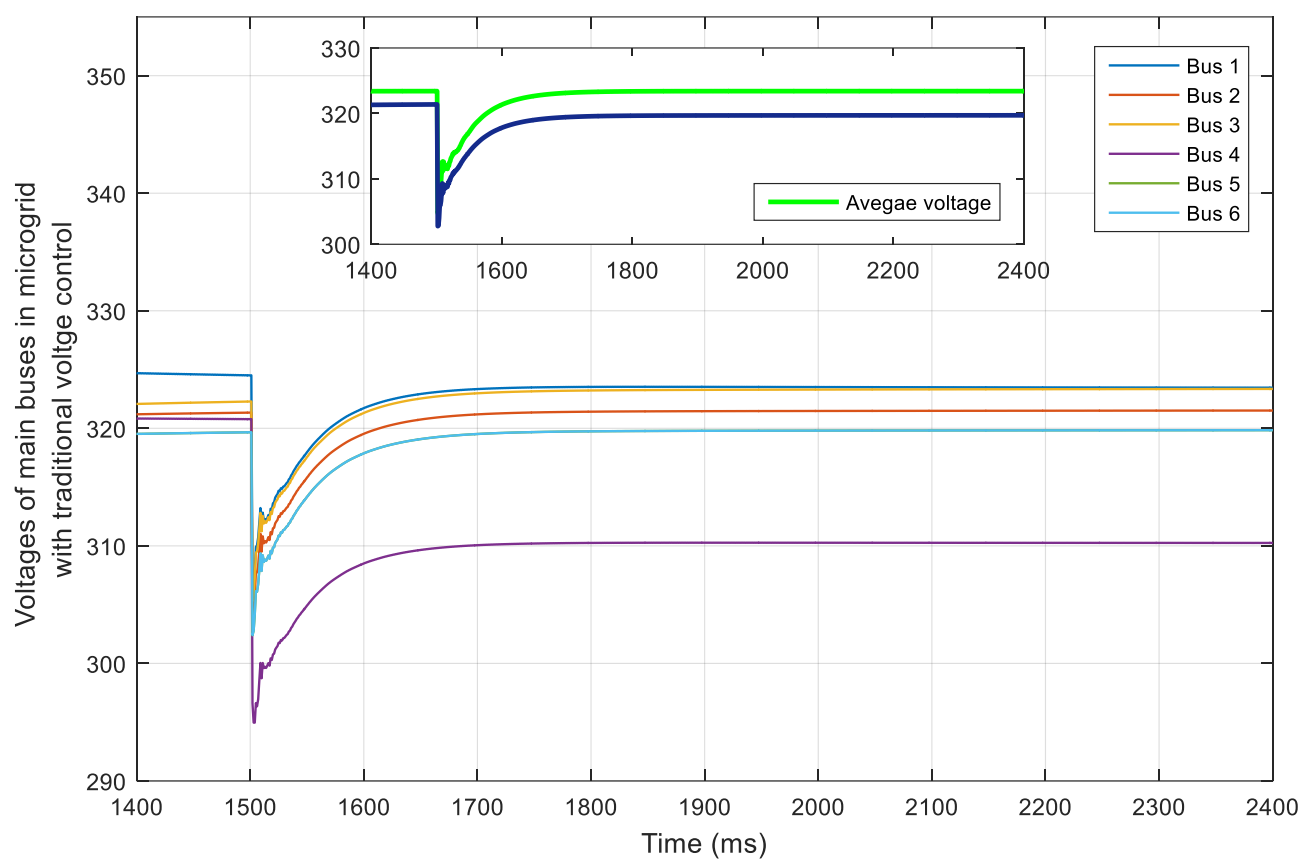

Figure 5.23: Voltages of main 6 buses with traditional voltage control (keeping VSI node voltages at 323.4 volt rms fixed) in case 1

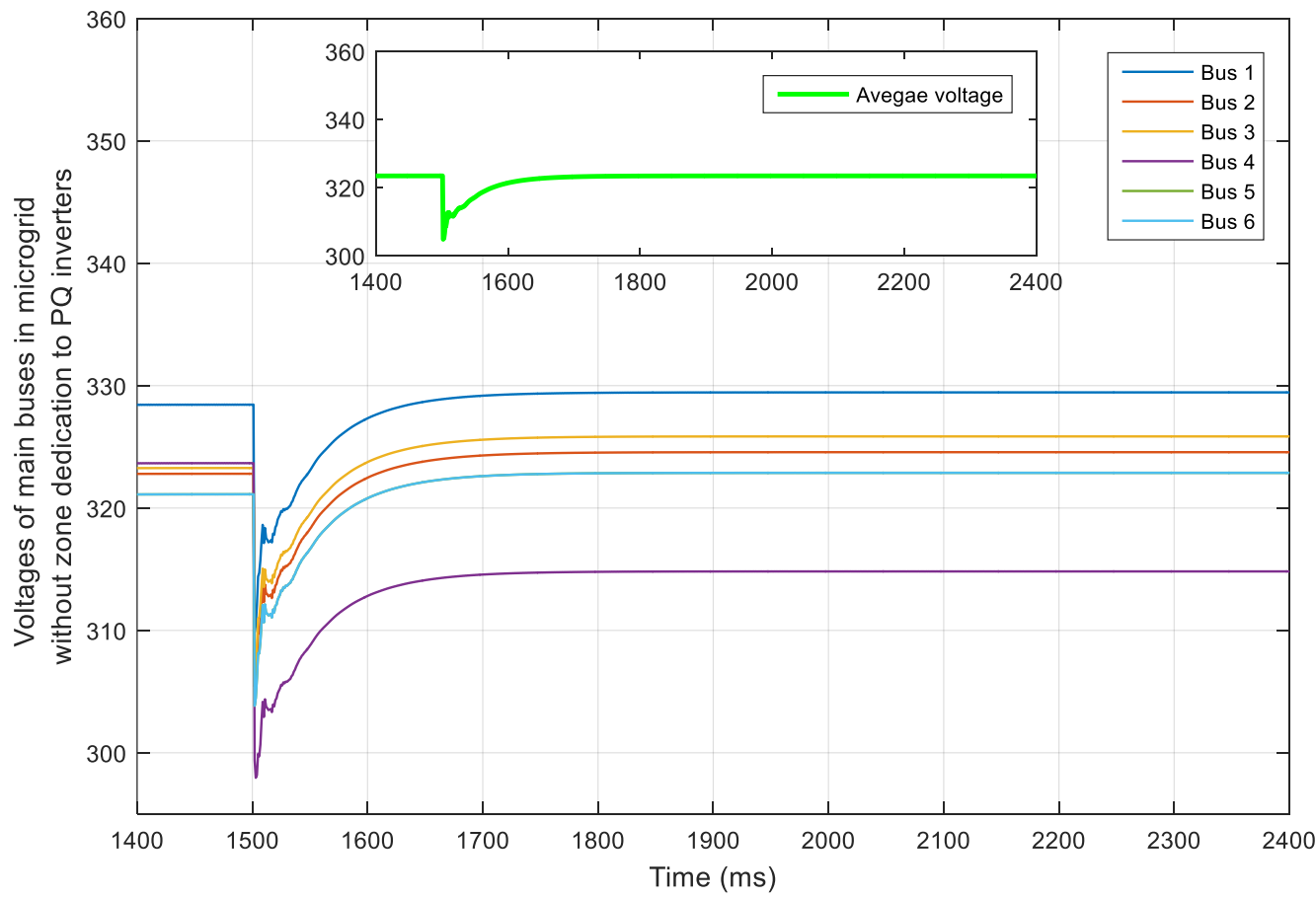

Figure 5.24: Voltages of main 6 buses with consensus-based voltage control (keeping the average voltages at 323.4 volt rms fixed) in case 1 


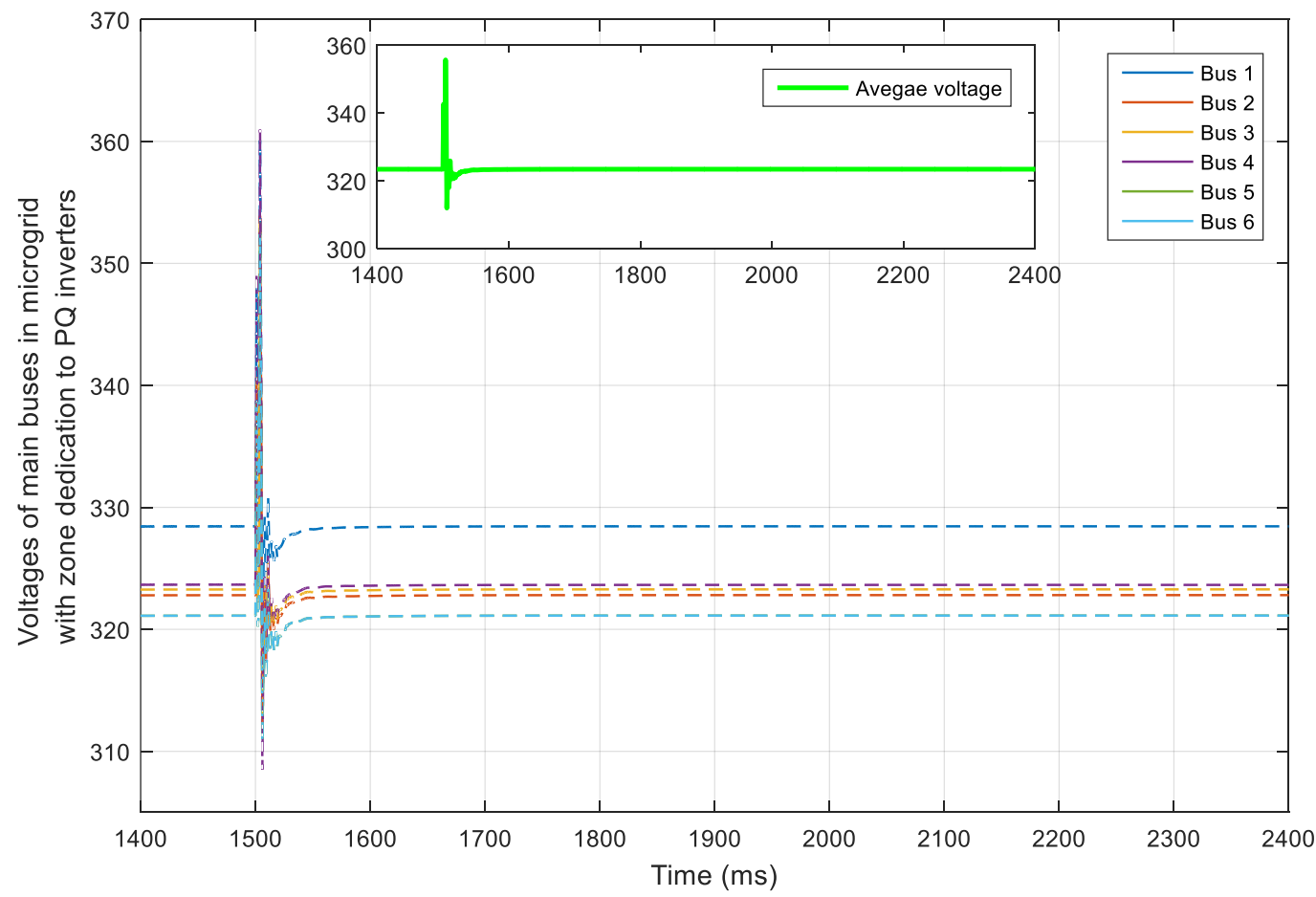

Figure 5.25: Voltages of main 6 buses with consensus-based voltage control along with zone dedication to PQ inverters

in case 1 

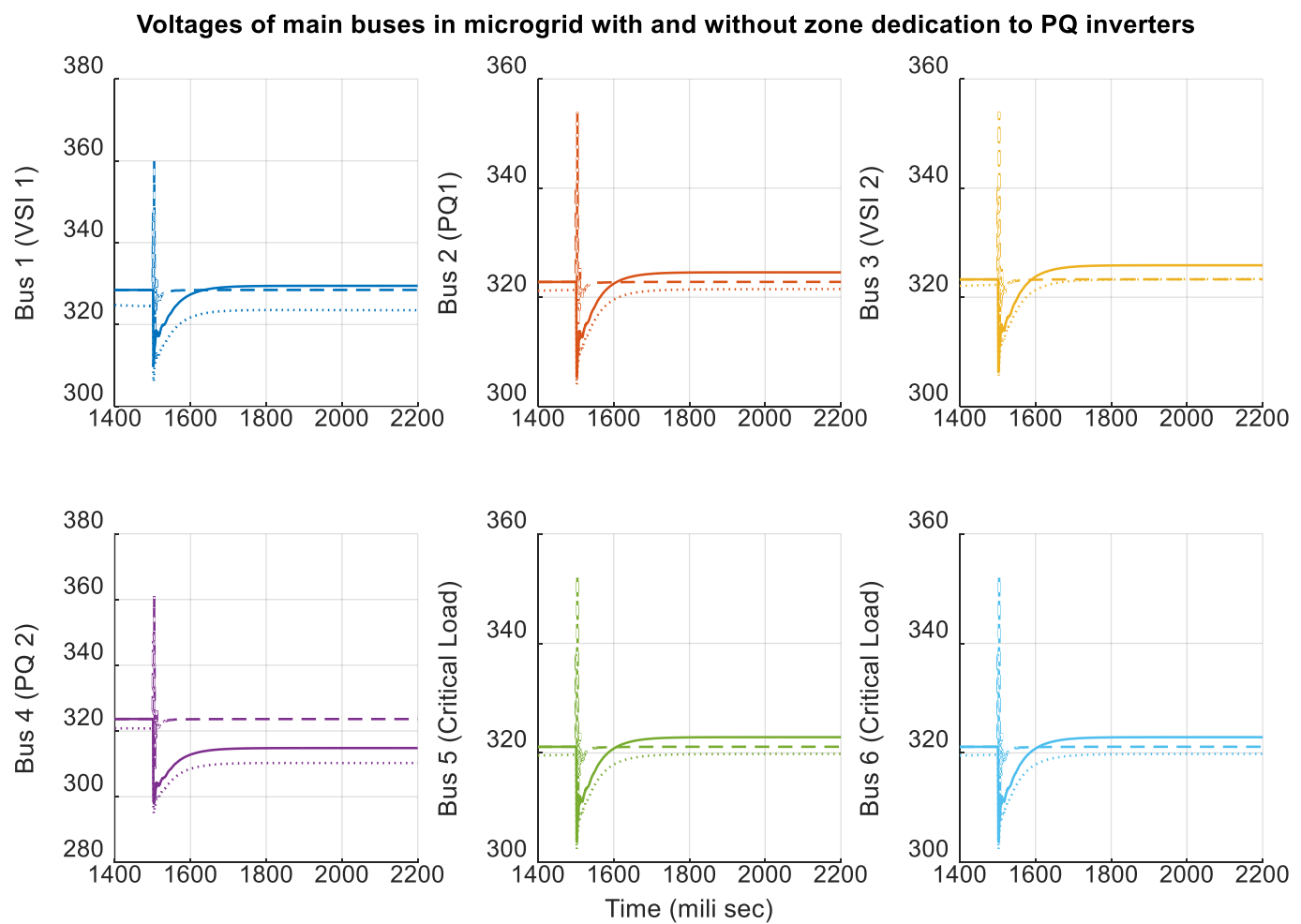

Figure 5.26: main bus voltages under different controller in case 1(dotted lines: traditional controller, solid line: consensus-based average voltage controller, broken line: zone dedicated method)

By comparing figures 5.24 and 5.25, it can be seen that the voltage at bus 4 experienced a drop due to the load change even though the average voltage regulator is doing its job correctly. Then, in figure 5.25, the zone dedication has been considered and the PQ inverters try to collect information from tie line agents and make sure that the load demand in their own area is fed through themselves, not the other inverters. This fact can be seen in figures 5.27 and 5.28, which show the active and reactive power produced by the four available inverters in the microgrid. Before implementing the zone definition method, both PQ inverters are providing constant power and the other two VSI inverters are taking care of the load demand; however, after giving the responsibility to the PQ attached to bus 4, it would be the only inverter changing its output after the demand increase. Figure 5.26 compares the voltages one-by-one to demonstrate the difference of all three controllers' effect in voltages. 
Active power of Inverters in microgrid with and without zone dedication to $P Q$ inverters
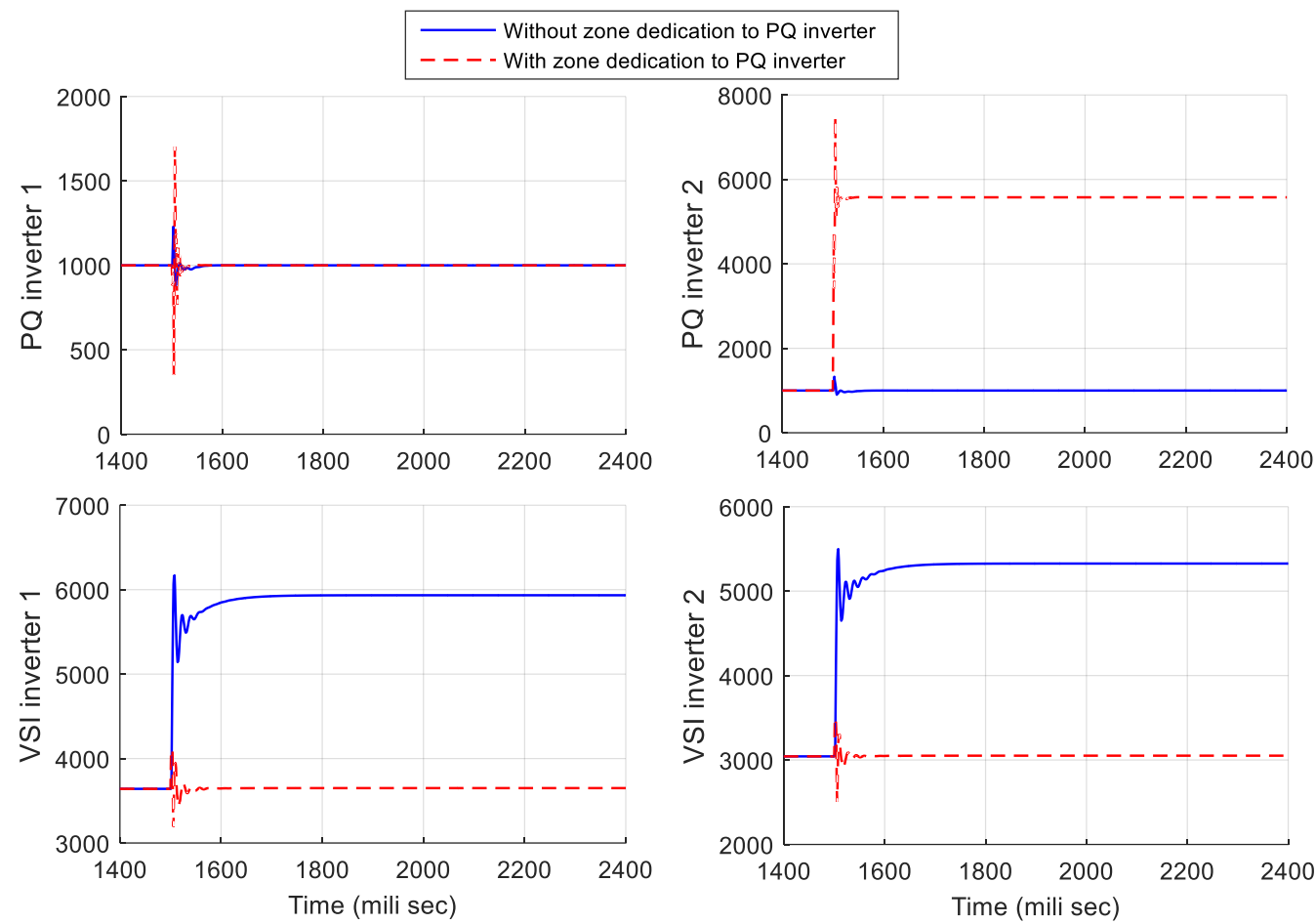

Figure 5.27: Active power produced by the four available inverters in the microgrid in case 1 
Reactive power of Inverters in microgrid with and without zone dedication to $P Q$ inverters

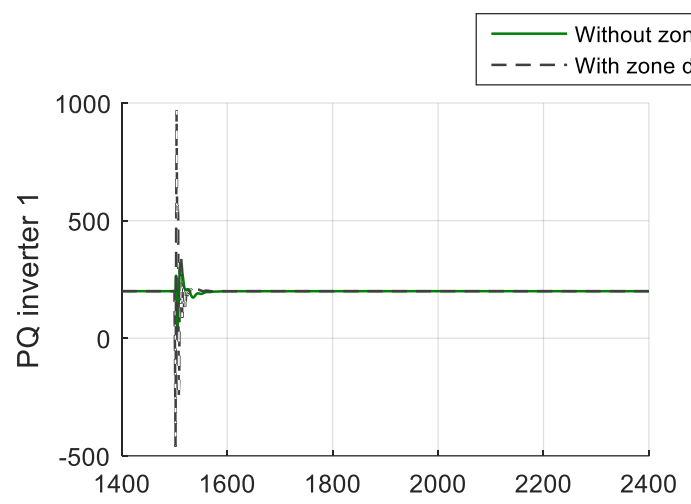
ededication to $P Q$ inverter
edication to $P Q$ inverter
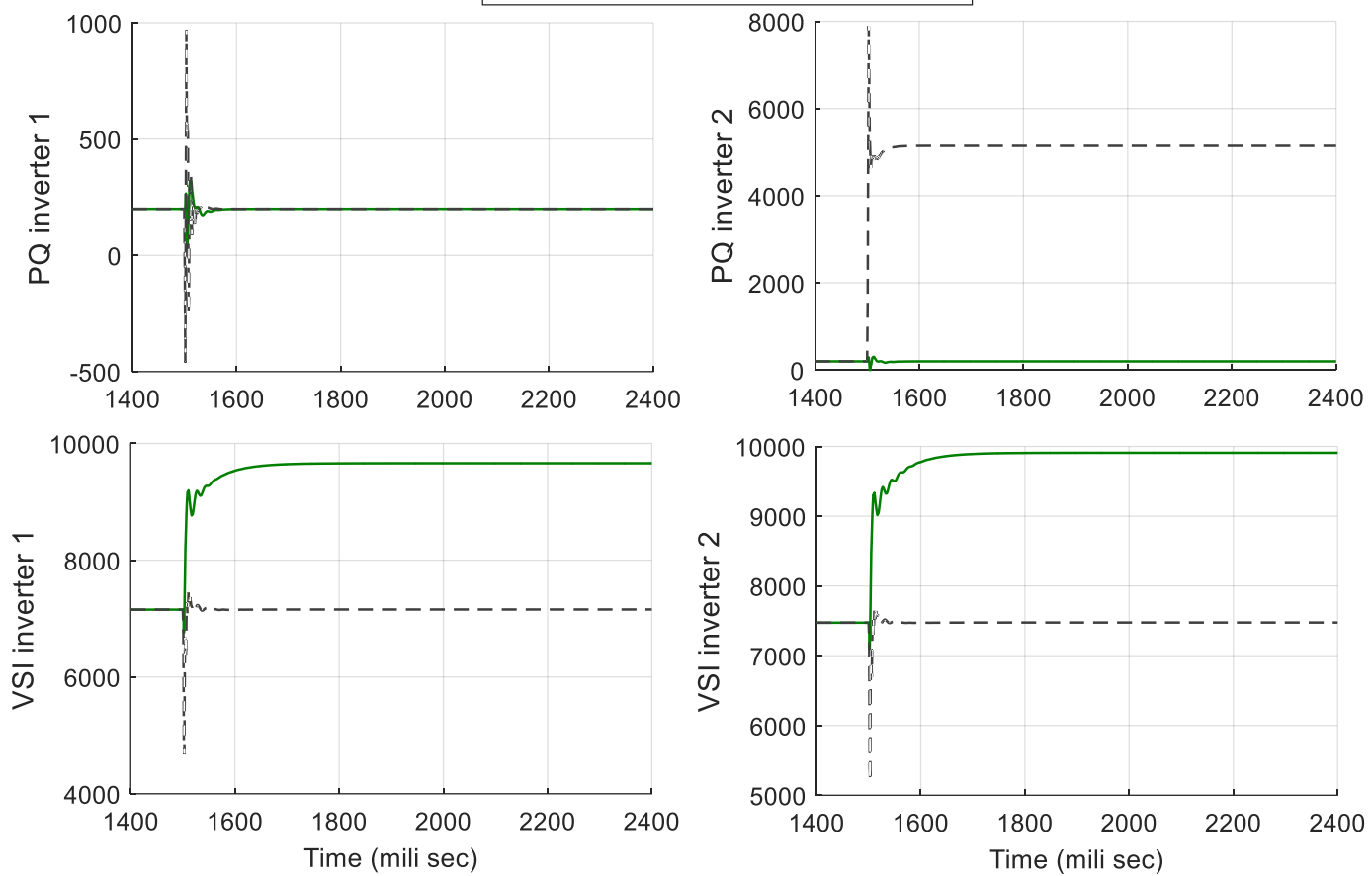

Figure 5.28: Reactive power produced by the four available inverters in the microgrid in case 1

Table 5.7 shows the steady state voltages at all six buses in all three situations of using different kinds of control schemes including a traditional controller and consensus-based controller with and without zone dedication. The mean value and variance are calculated for all circumstances. It can be seen that the variance of voltages is reduced significantly through the contribution of PQ inverters in supplying the load demand comparing to the consensus-based control alone. Figure 5.29 shows the mentioned table on a graph. So, it could be more sensible as a voltage profile curve.

TABLE 5.7

STEADY STATE VOLTAGE VALUE WITH AND WITHOUT ZONE DEDICATION TO PQ INVERTERS FOR LOAD INCREASE IN ZONE 4

\begin{tabular}{|c|c|c|c|c|c|c|c|c|}
\hline & $\begin{array}{c}\text { BUS } 1 \\
\text { VOLTAGE }\end{array}$ & $\begin{array}{c}\text { BUS } 2 \\
\text { VOLTAGE }\end{array}$ & $\begin{array}{c}\text { BUS } 3 \\
\text { VOLTAGE }\end{array}$ & $\begin{array}{c}\text { Bus } 4 \\
\text { VOLTAGE }\end{array}$ & $\begin{array}{c}\text { Bus } 5 \\
\text { VOLTAGE }\end{array}$ & $\begin{array}{c}\text { Bus } 6 \\
\text { vOLTAGE }\end{array}$ & MEAN & VARIANCE \\
\hline TRADITIONAL CONTROL & 323.4 & 321.5 & 323.4 & 310.25 & 319.83 & 319.83 & 319.70 & 120.2 \\
\hline $\begin{array}{c}\text { WITHOUT ZONE DEDICATION TO } \\
\text { PQ INVERTERS }\end{array}$ & 329.44 & 324.56 & 325.85 & 314.81 & 322.87 & 322.87 & 323.40 & 118.179 \\
\hline $\begin{array}{l}\text { WITH ZONE DEDICATION TO PQ } \\
\text { INVERTERS }\end{array}$ & 328.44 & 322.81 & 323.27 & 323.64 & 321.127 & 321.126 & 323.40 & 36.16 \\
\hline
\end{tabular}




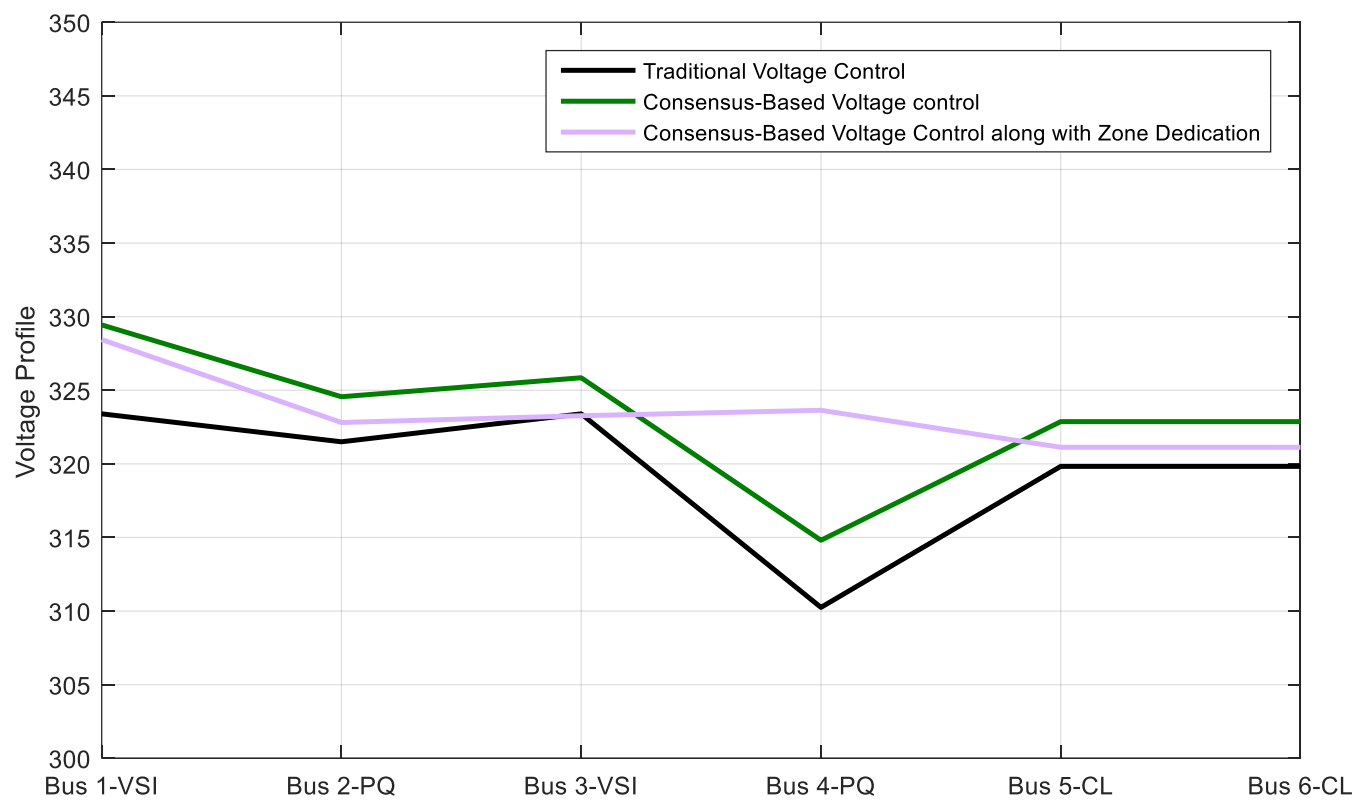

Figure 5.29: Voltage profile of main buses with three different control scheme (traditional, consensus-based with and

without zone dedication) in case 1

\section{2) Case two}

The second case is almost similar to the first case, only the load increase has been changed to load decrease. The purpose of this scenario is to examine the applicability of zone dedication when the load demand is decreased by the same value as case one. In this scenario, the active and reactive load decrease is equal to $4.5 \mathrm{k}$ watt and $5 \mathrm{k}$ var respectively and it happens at $\mathrm{t}=2.5 \mathrm{sec}$. The results for both having and not having zones is presented here in figures 5.31 and 5.30 respectively. Figure 5.32 compares the voltages one-byone to demonstrate the difference of both controllers' effect on voltages.

Figure 5.33 and 5.34 shows the active and reactive power produced by the four available inverters in the microgrid before and after implementing the zone definition method. 


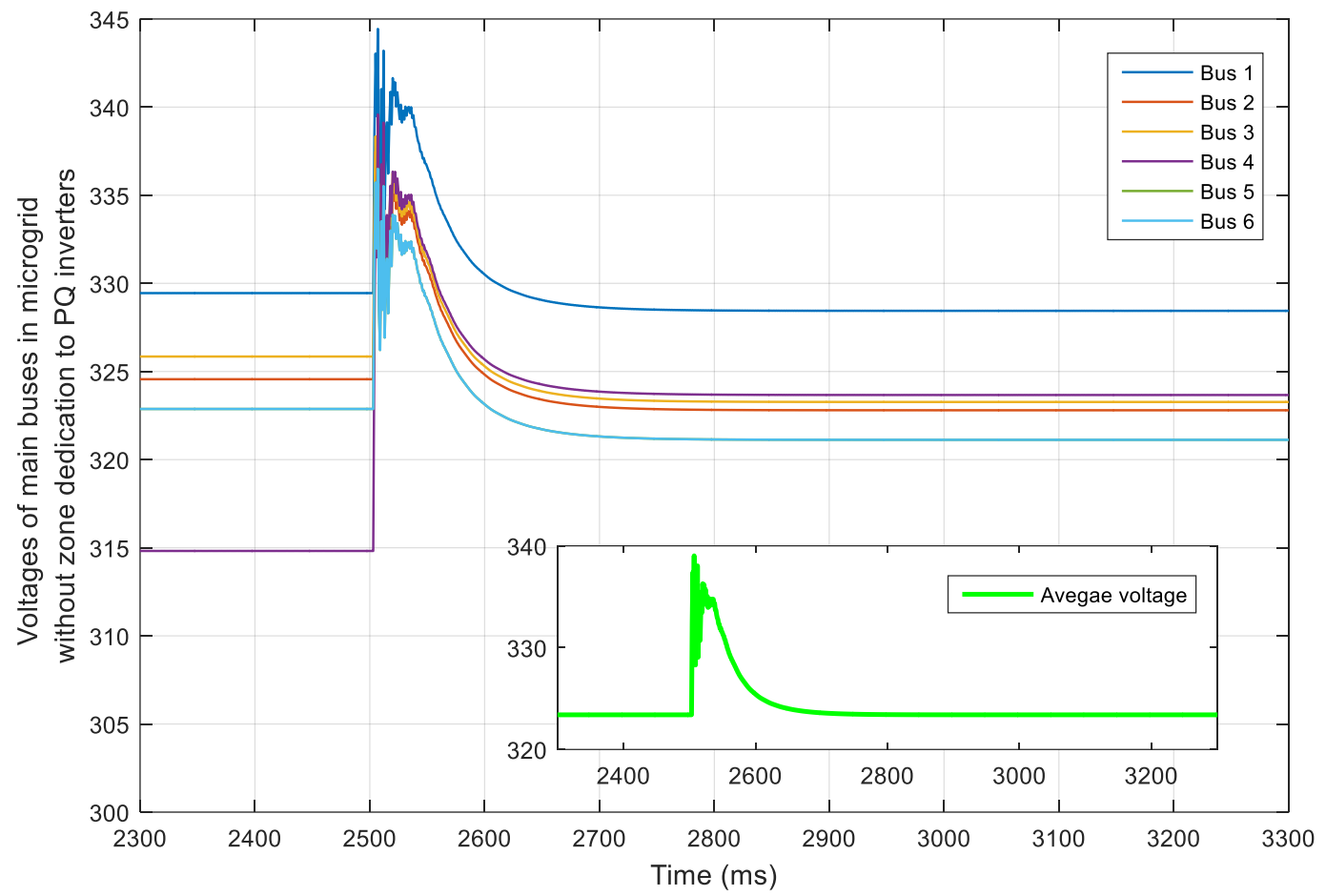

Figure 5.30: Voltages of main 6 buses with consensus-based voltage control (keeping the average voltages at 323.4 volt rms fixed) in case 2

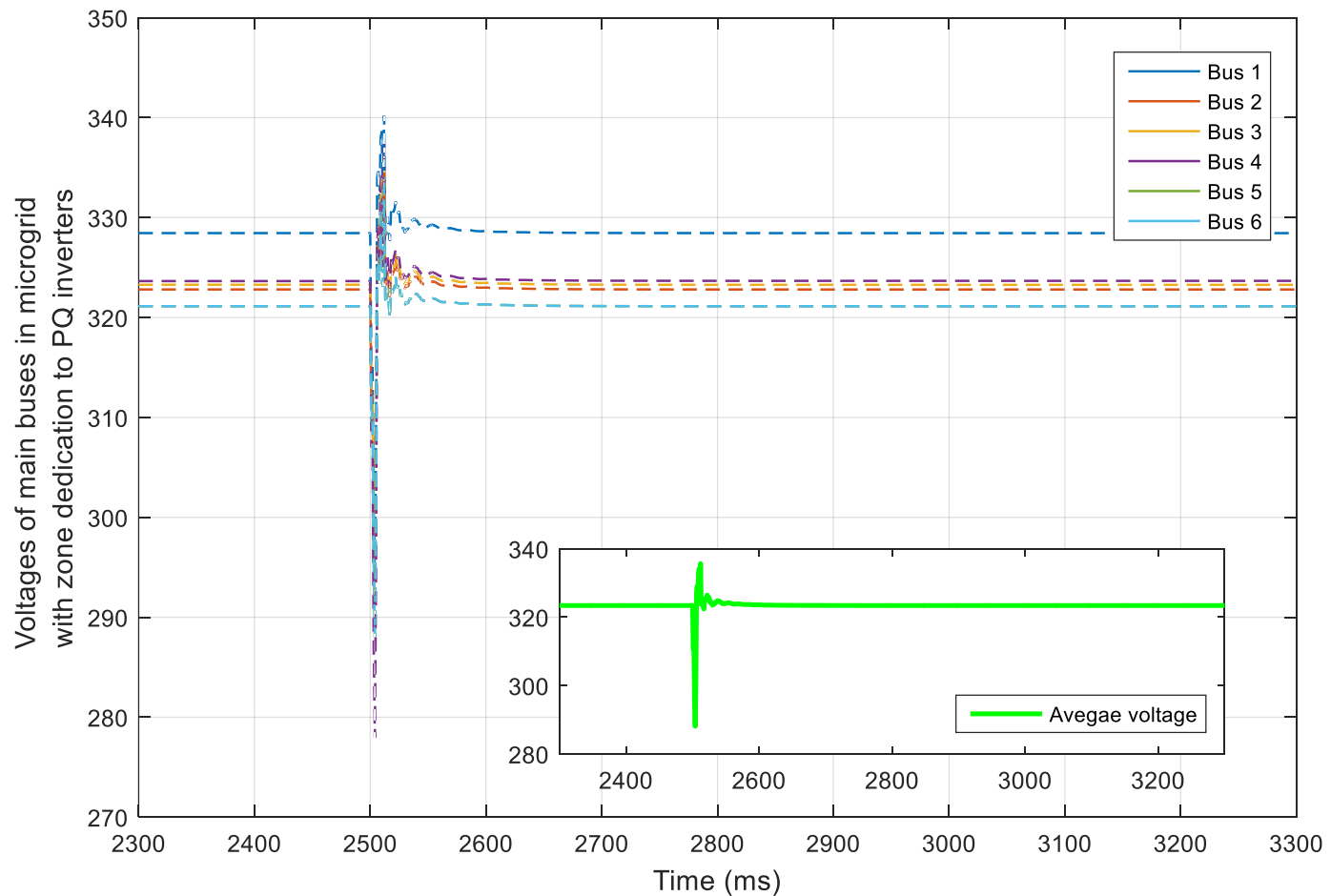

Figure 5.31: Voltages of main 6 buses with consensus-based voltage control along with zone dedication to PQ inverters in case 2 

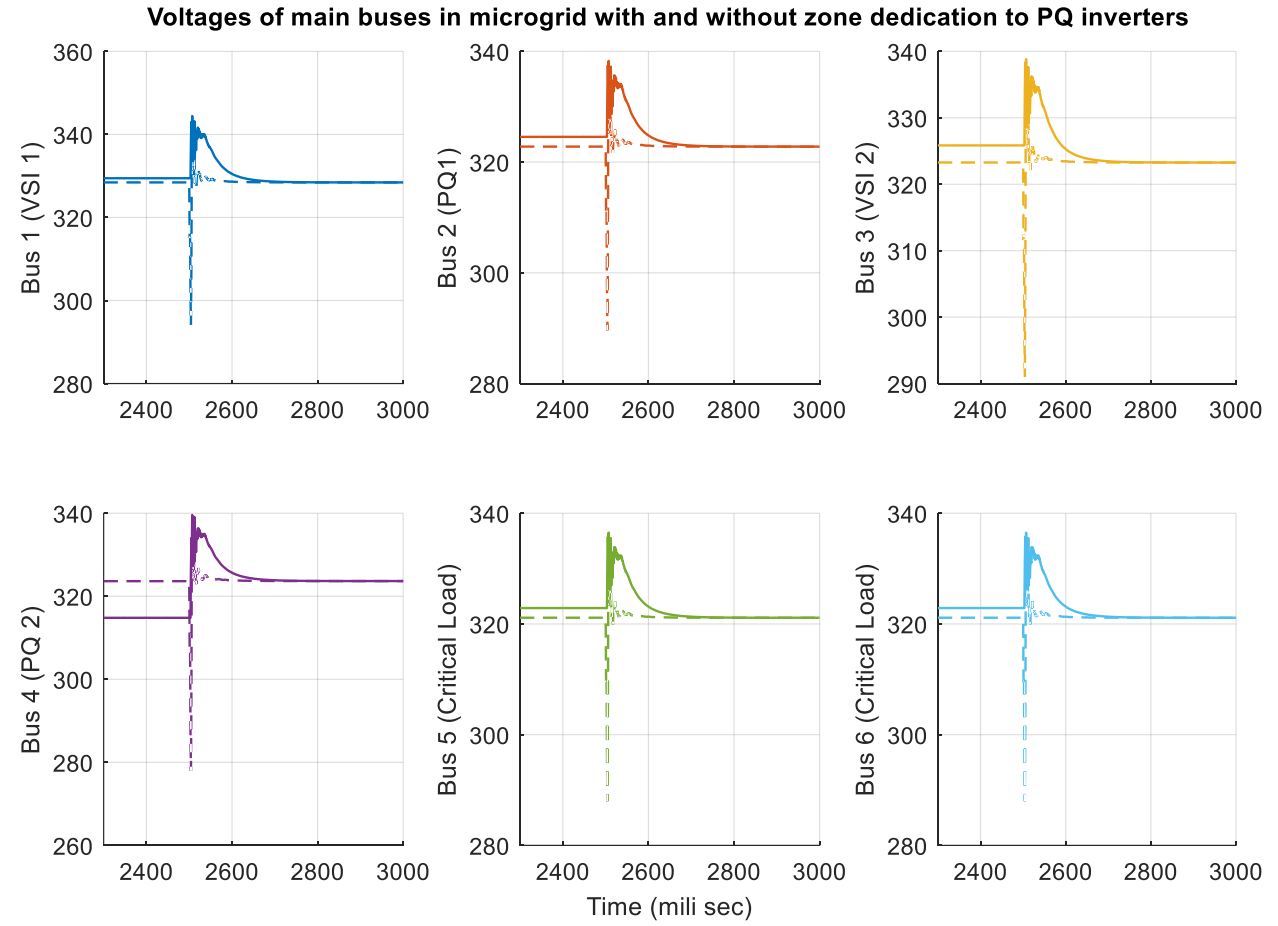

Figure 32: main bus voltages under different controller in case 2 (solid line: consensus-based average voltage controller, broken line: zone dedicated method) 
Active power of Inverters in microgrid with and without zone dedication to $P Q$ inverters

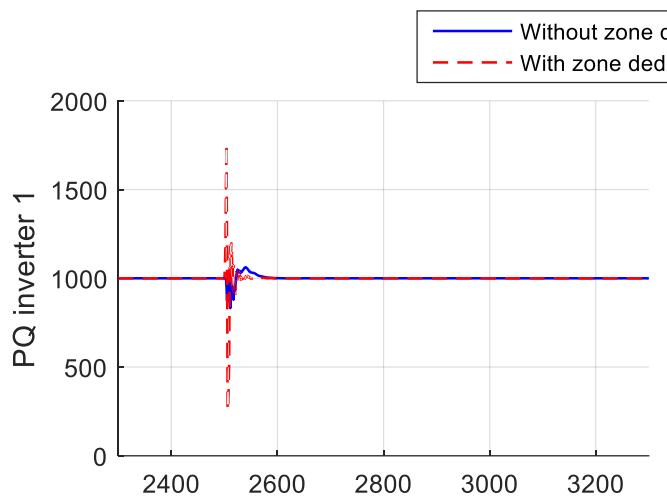
dication to $P Q$ inverter
cation to $P Q$ inverter
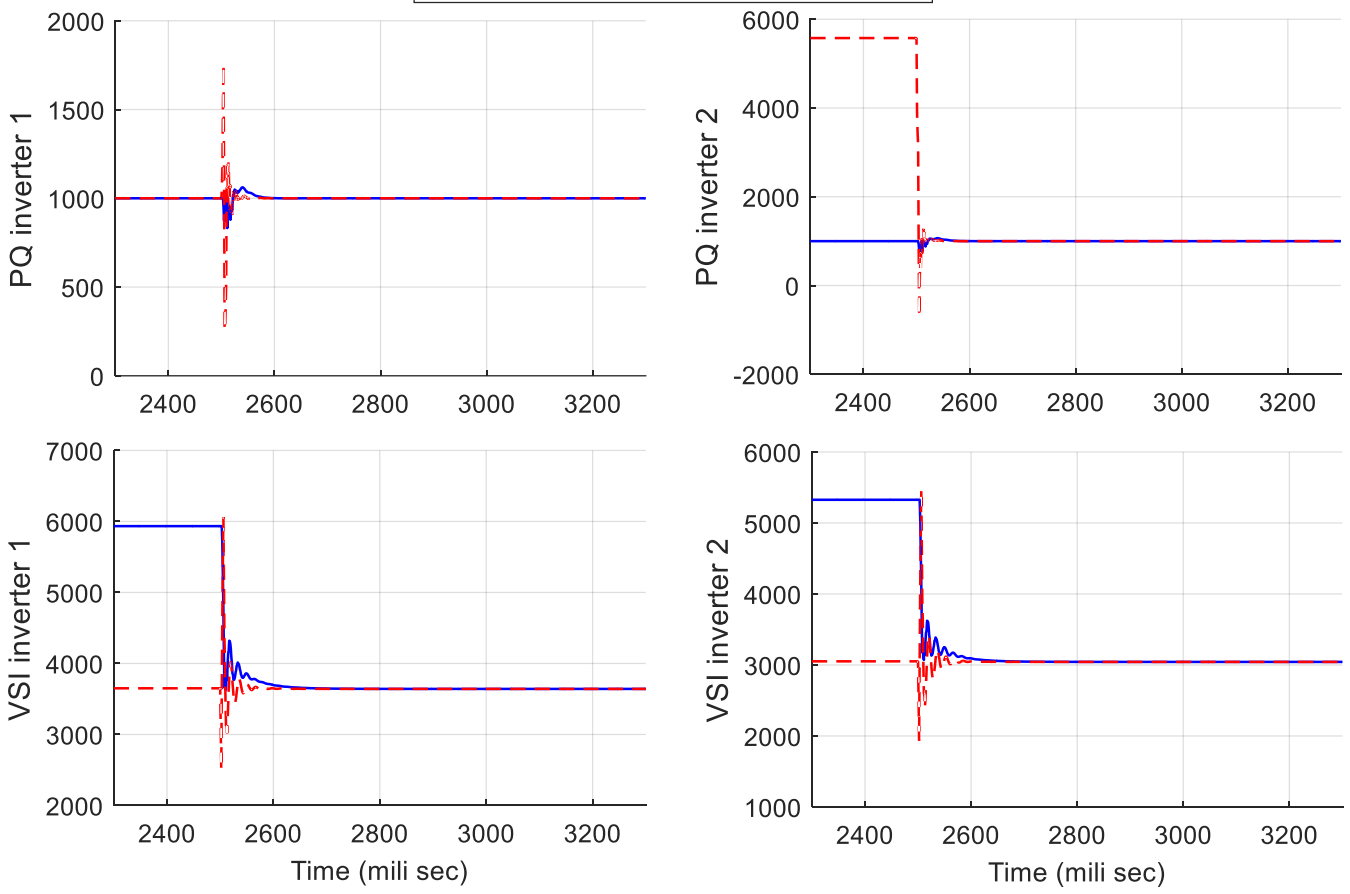

Figure 5.33: Active power produced by the four available inverters in the microgrid in case 2 

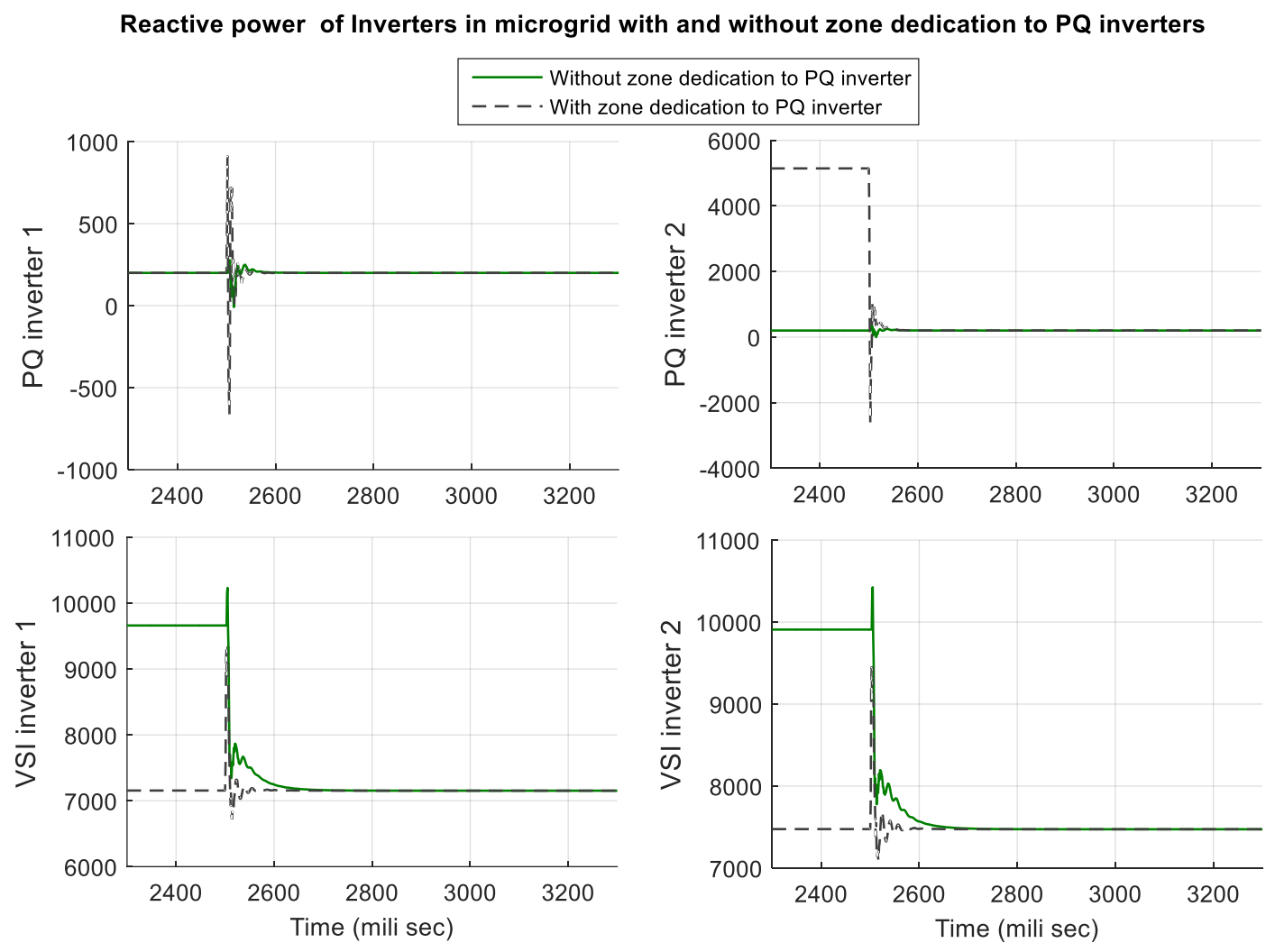

Figure 5.34: Reactive power produced by the four available inverters in the microgrid in case 2

\section{3) Case three:}

The third case is about a load change in zone 2. The difference in this case is related to the distance between the location of the demanded load and the responsible PQ inverter of the zone. In previous cases, the load was attached to the same bus as the PQ inverter; consequently, it had a high sensitivity to that specific inverter. However, if there is a long distance between the demanded load and the PQ inverter, the zone dedication might not be as effective as previous cases but it would still be better than the traditional or the consensus-based only algorithm. The reason is obvious; the bus voltage with the load change still has the most sensitivity to the corresponding PQ inverter even though it is not too much.

Figure 5.35 shows the system and load change scenario inside. This load has been added to the system at $\mathrm{t}=1.5 \mathrm{sec}$ and then it is being disconnected at $\mathrm{t}=2.5 \mathrm{sec}$. The results for all three control scheme-namely traditional control, consensus-based only average control and consensus-based average control with zone dedication to PQ inverters - are presented here in figures 5.36, 5.37 and 5.38 respectively. 
Figure 5.39 compares the voltages one-by-one to demonstrate the difference of all of these controllers' effect on voltages, then figures 5.40 and 5.41 show the active and reactive power produced by the four available inverters in the microgrid before and after implementing the zone definition method.

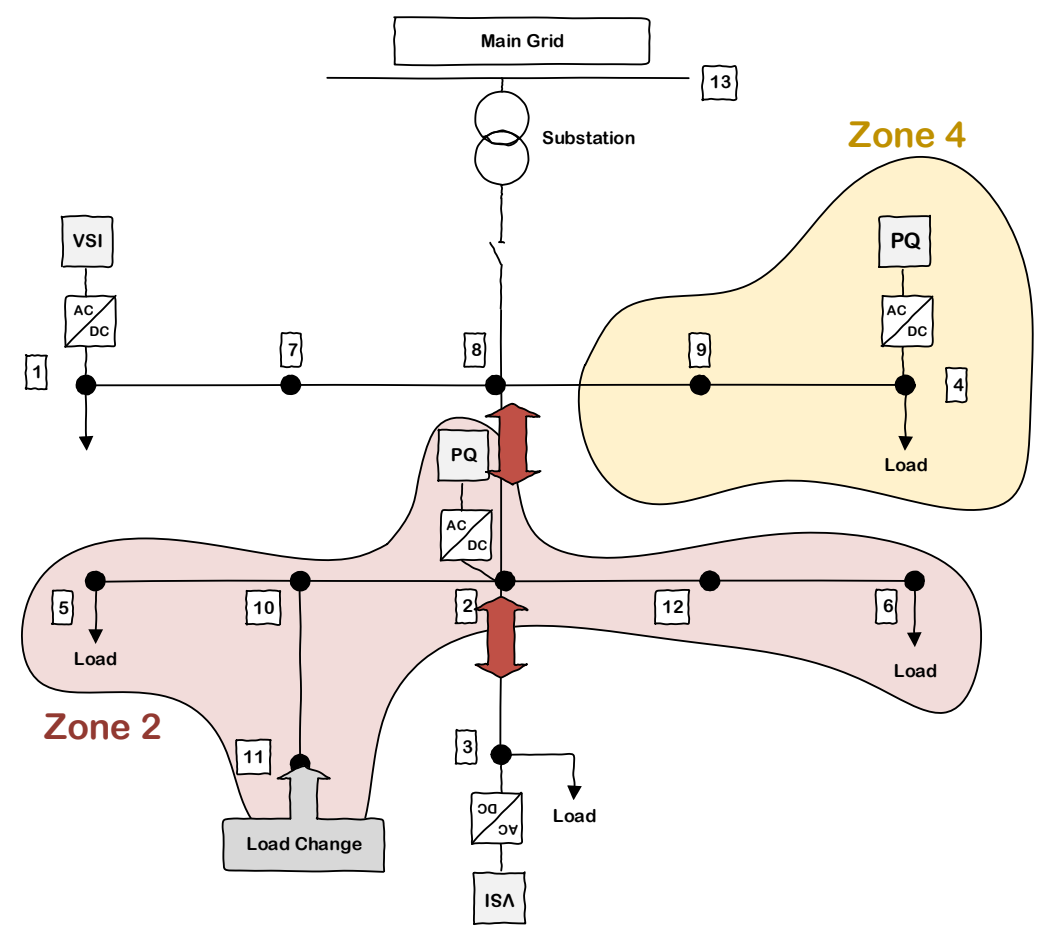

Figure 5.35: Load change location in case three 


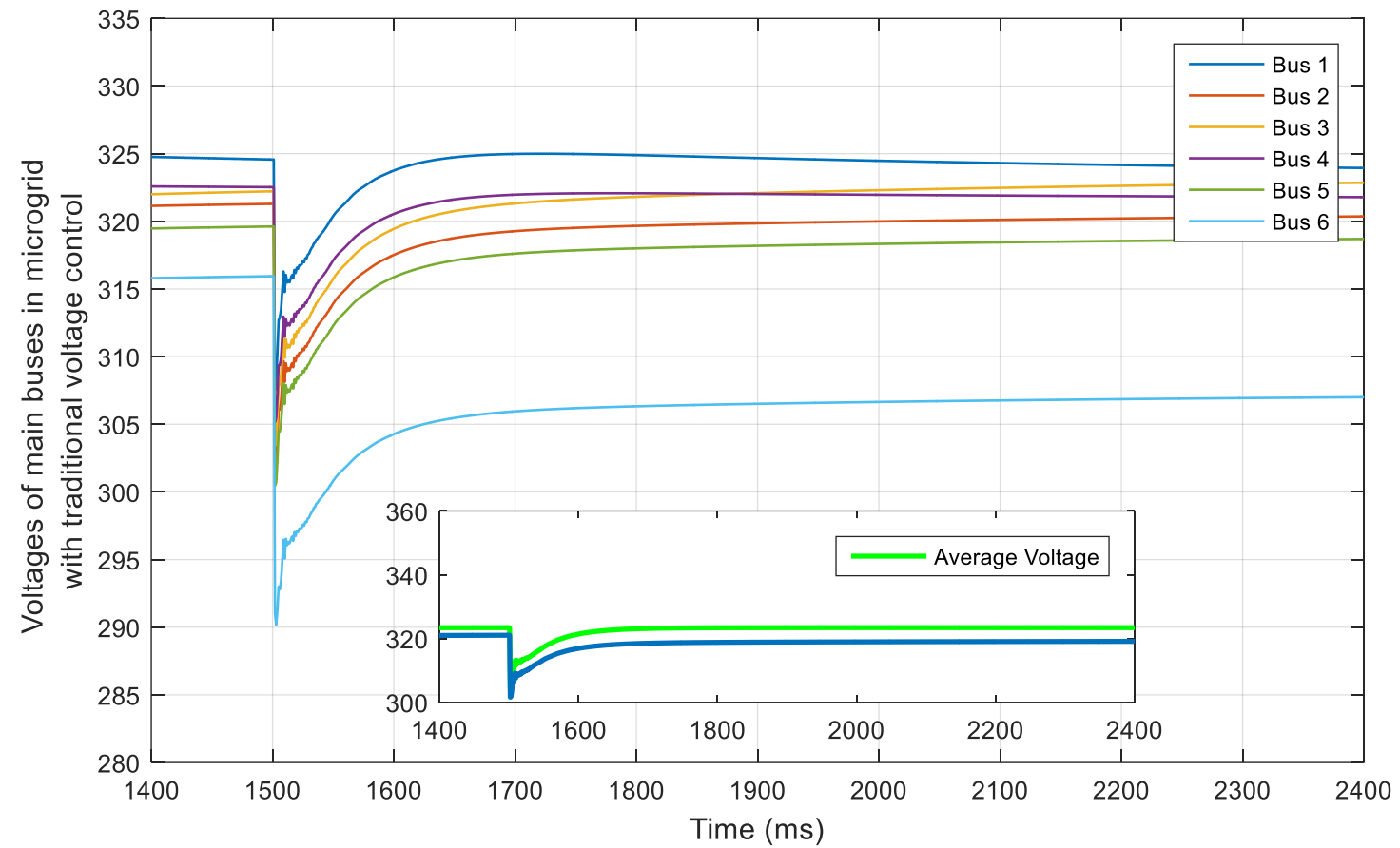

Figure 5.36: Voltages of main 6 buses with traditional voltage control (keeping VSI node voltages at 323.4 volt rms fixed) in case 3 


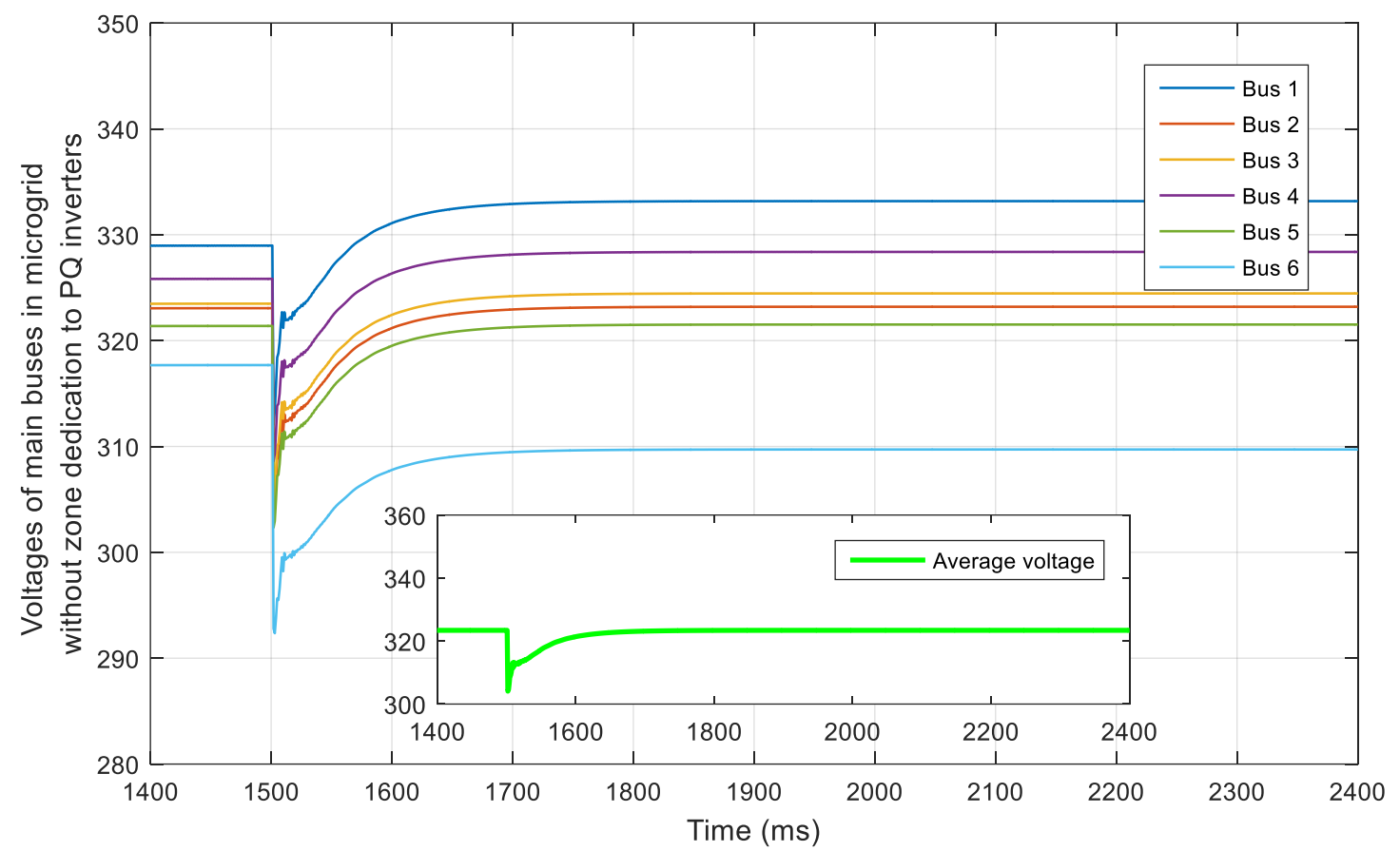

Figure 5.37: Voltages of main 6 buses with consensus-based voltage control (keeping the average voltages at 323.4 volt rms fixed) in case 3 


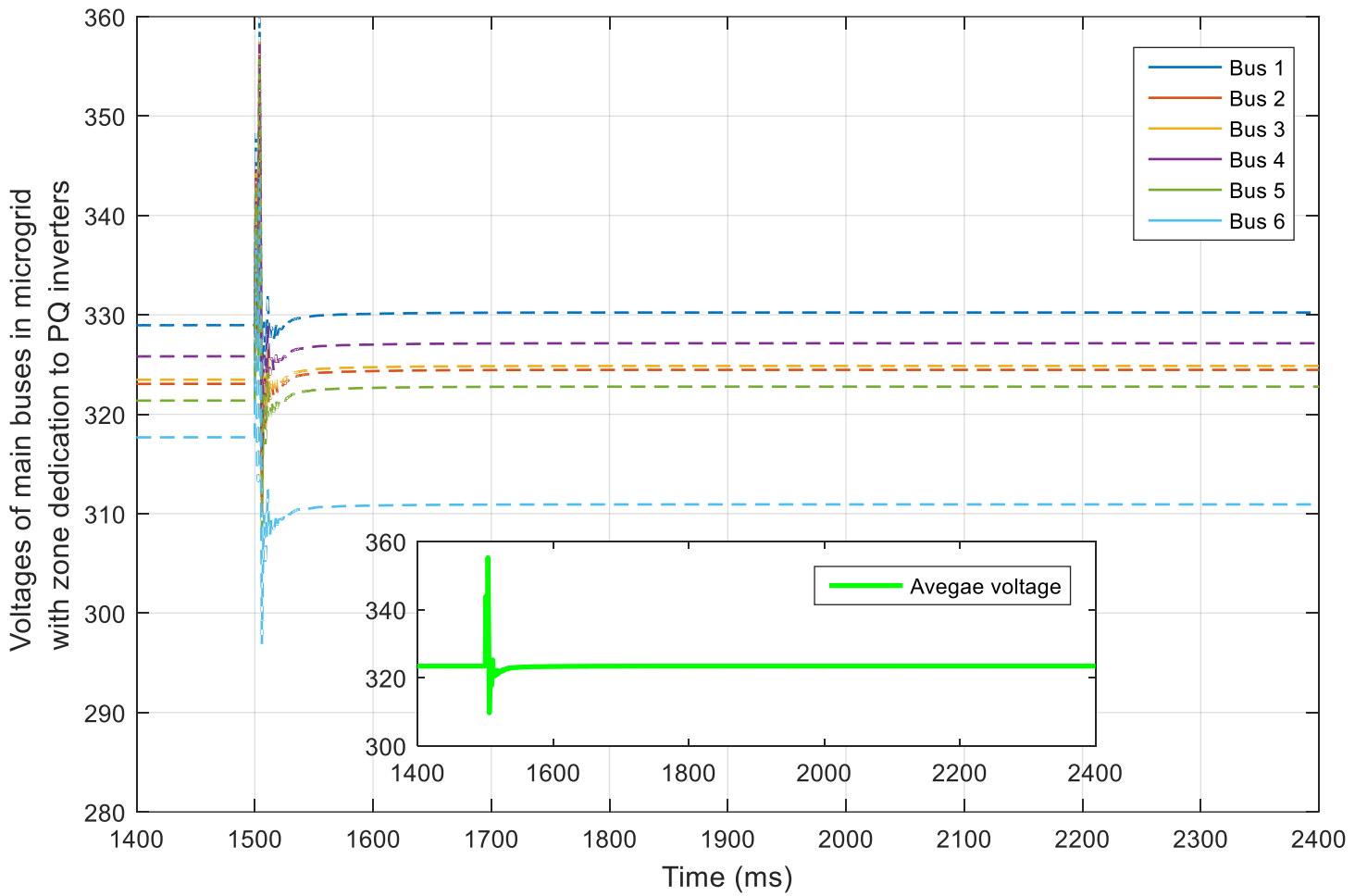

Figure 5.38: Voltages of main 6 buses with consensus-based voltage control along with zone dedication to PQ inverters in case 3 

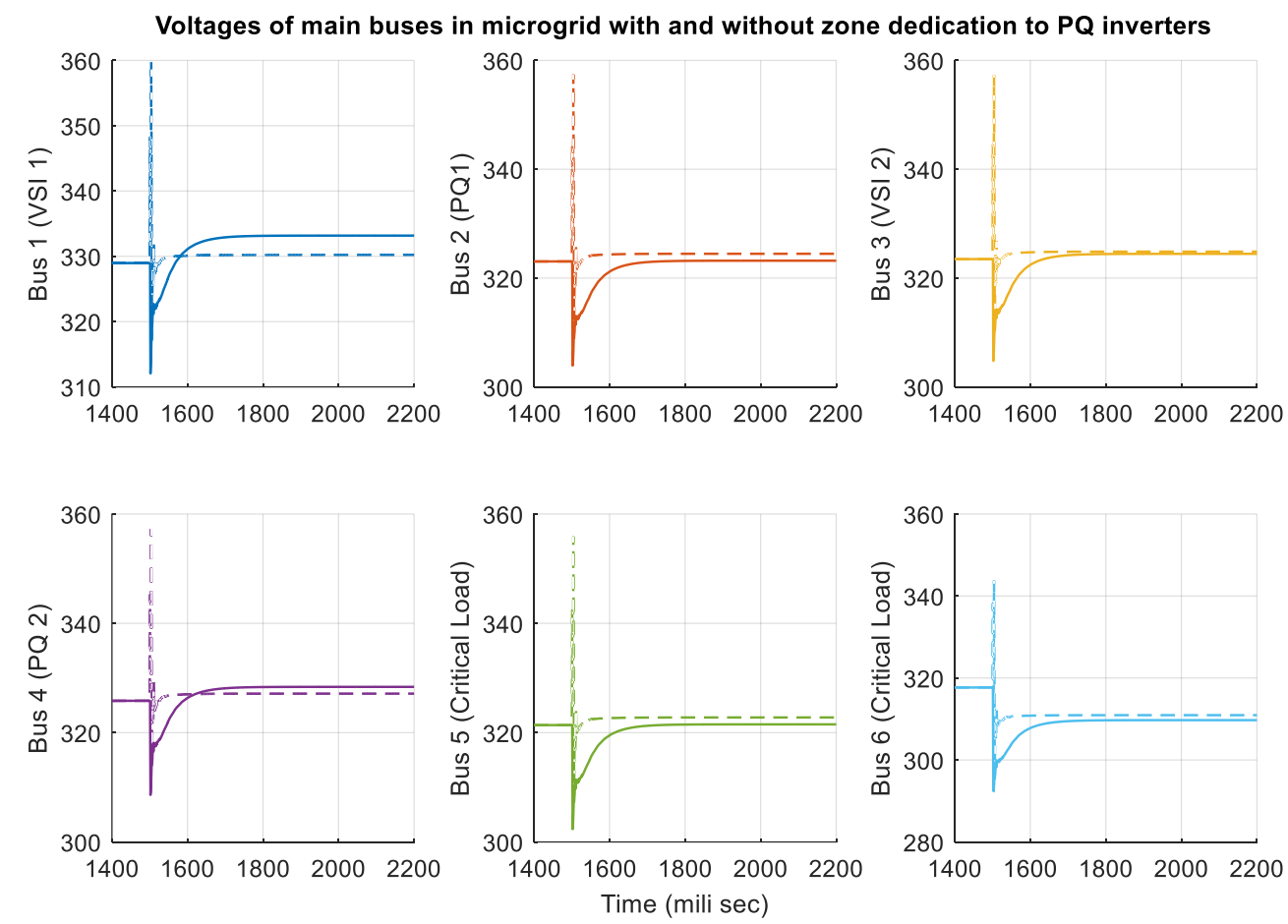

Figure 39: main bus voltages under different controller in case 3 (solid line: consensus-based average voltage controller, broken line: zone dedicated method)

Active power of Inverters in microgrid with and without zone dedication to $P Q$ inverters

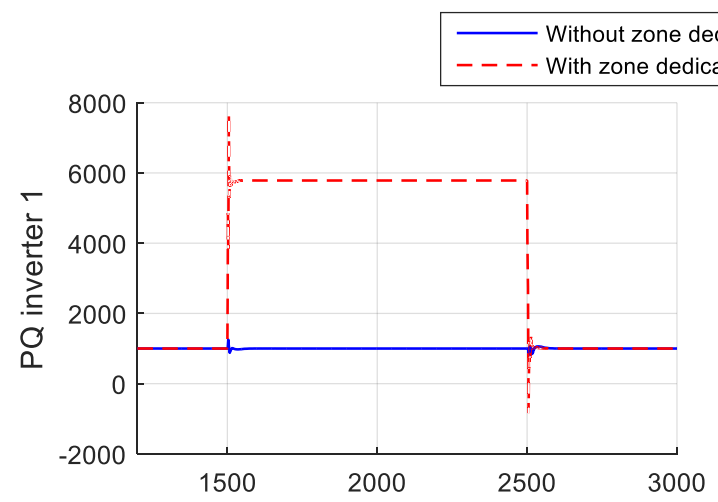

$$
\begin{aligned}
& \text { dication to } P Q \text { inverter } \\
& \text { ation to } P Q \text { inverter }
\end{aligned}
$$
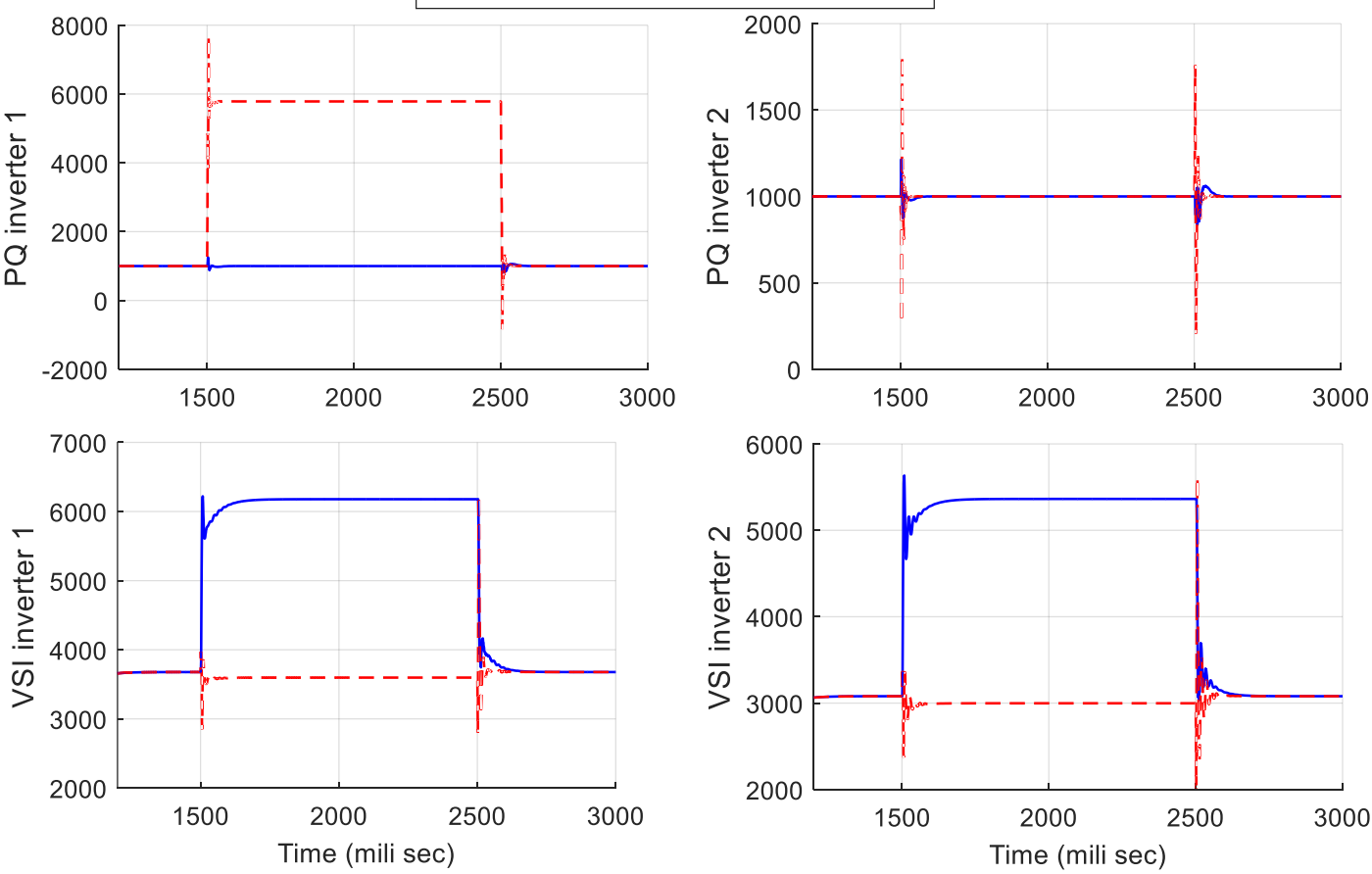

Figure 5.40: Active power produced by the four available inverters in the microgrid in case 3 


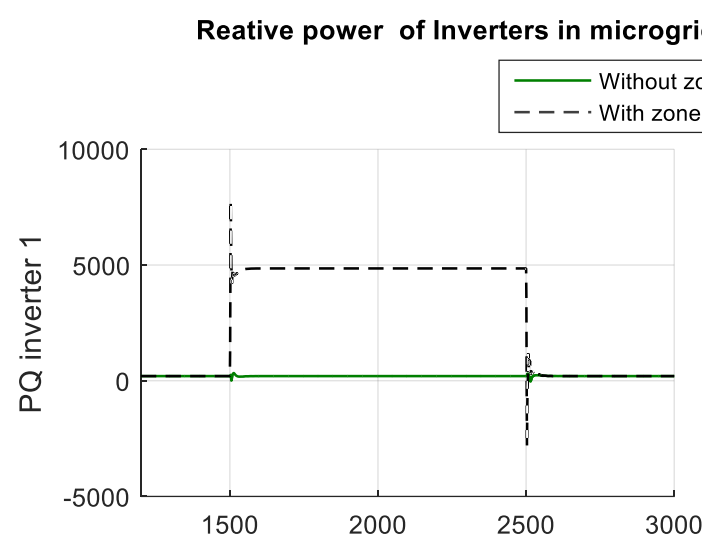

with and without zone
dedication to $P Q$ inverter
dedion to $P Q$ inverter
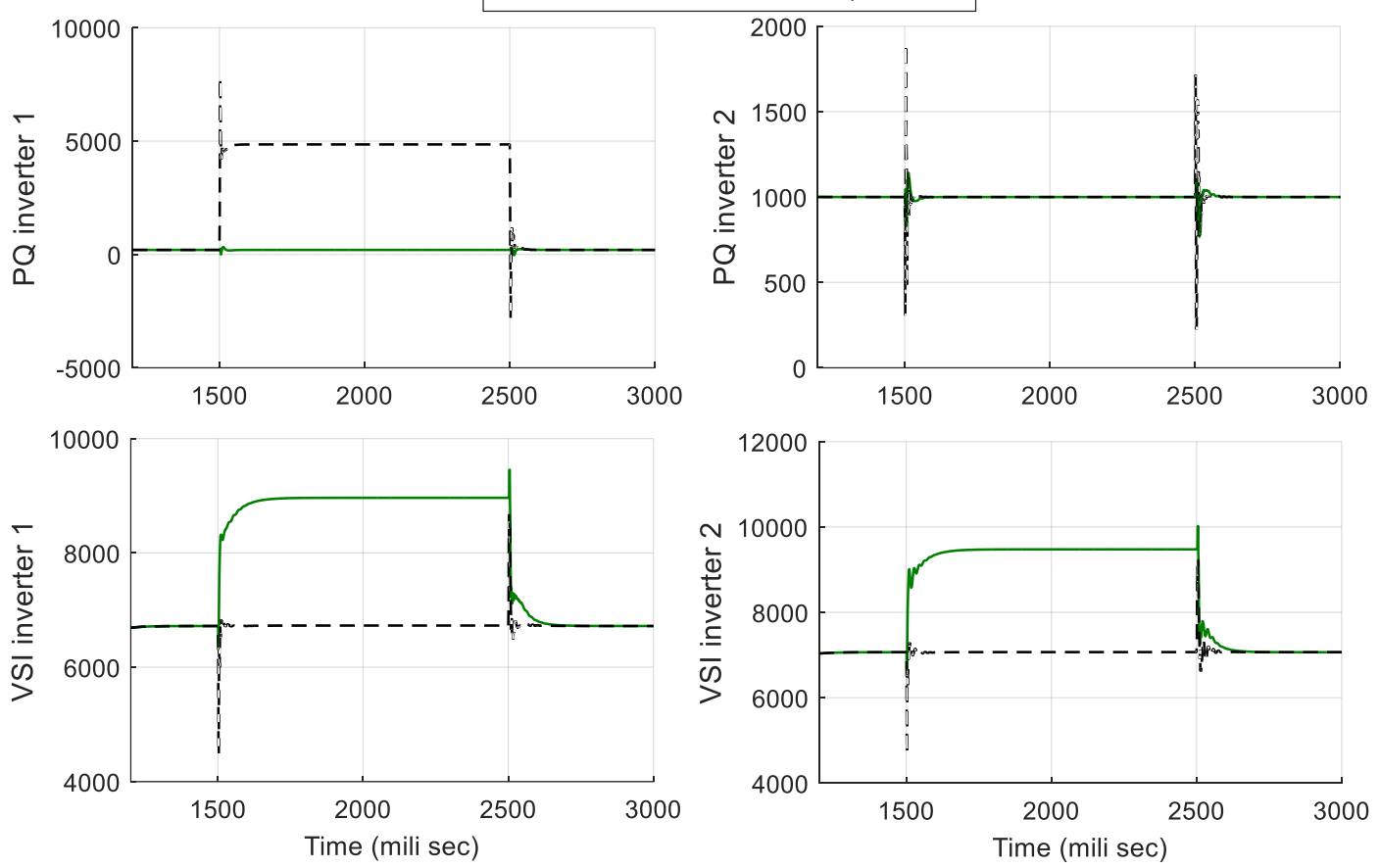

Figure 5.41: Reactive power produced by the four available inverters in the microgrid in case 3

Table 5.8 shows the steady state voltages at all six buses in all three situations of using different kinds of control schemes including a traditional controller and consensus-based controller with and without zone dedication. The mean value and variance are calculated for all circumstances.

By comparing the consensus-based voltage control with and without zone dedication, it can be observed that the variance of voltages is reduced slightly and is not as significant of the first case, but, it still improves the variance, which means it can increase the lowest voltage of microgrid. Figure 5.42 shows the mentioned table on a graph. So, it could be more sensible as a voltage profile curve.

TABLE 5.8

STEADY STATE VOLTAGE VALUE WITH AND WITHOUT ZONE DEDICATION TO PQ INVERTERS FOR LOAD INCREASE IN ZONE 2

\begin{tabular}{|c|c|c|c|c|c|c|c|c|}
\hline & $\begin{array}{c}\text { BUS } 1 \\
\text { VOLTAGE }\end{array}$ & $\begin{array}{c}\text { BUS } 2 \\
\text { VOLTAGE }\end{array}$ & $\begin{array}{c}\text { Bus } 3 \\
\text { VOLTAGE }\end{array}$ & $\begin{array}{c}\text { Bus } 4 \\
\text { VOLTAGE }\end{array}$ & $\begin{array}{c}\text { Bus } 5 \\
\text { VOLTAGE }\end{array}$ & $\begin{array}{c}\text { Bus } 6 \\
\text { VOLTAGE }\end{array}$ & MEAN & VARIANCE \\
\hline TRADITIONAL CONTROL & 323.4 & 320.42 & 323.4 & 321.75 & 318.7 & 306.91 & 319.09 & 194.5 \\
\hline $\begin{array}{c}\text { WITHOUT ZONE DEDICATION TO } \\
\text { PQ INVERTERS }\end{array}$ & 333.17 & 323.195 & 324.45 & 328.375 & 321.52 & 309.71 & 323.40 & 312.29 \\
\hline $\begin{array}{l}\text { WITH ZONE DEDICATION TO PQ } \\
\text { INVERTERS }\end{array}$ & 330.23 & 324.47 & 324.86 & 327.14 & 322.78 & 310.94 & 323.40 & 219.54 \\
\hline
\end{tabular}




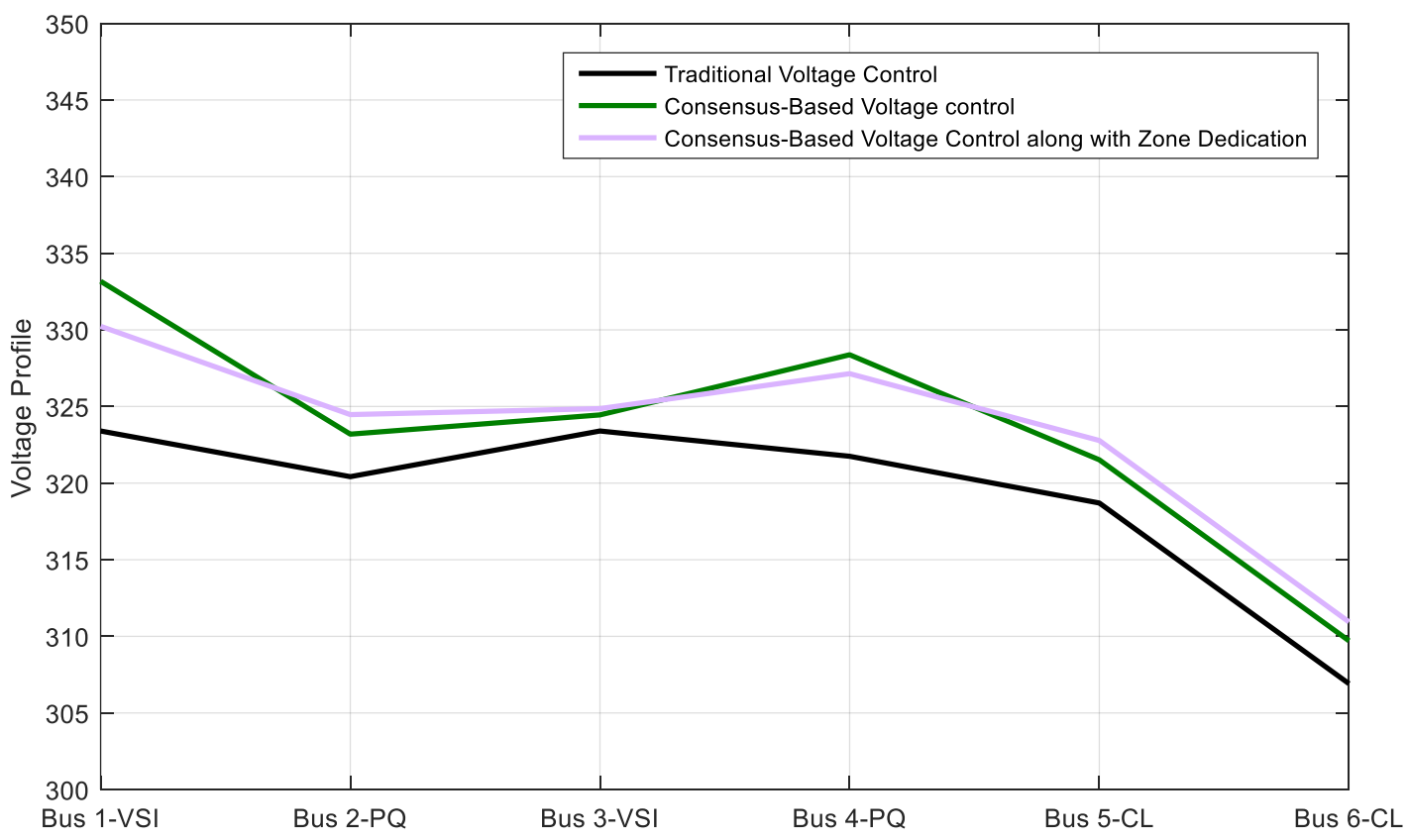

Figure 5.42: Voltage profile of main buses with three different control scheme (traditional, consensus-based with and without zone dedication) in case 3 


\section{Chapter 6}

Appendices 


\subsection{PSCAD MODELING}
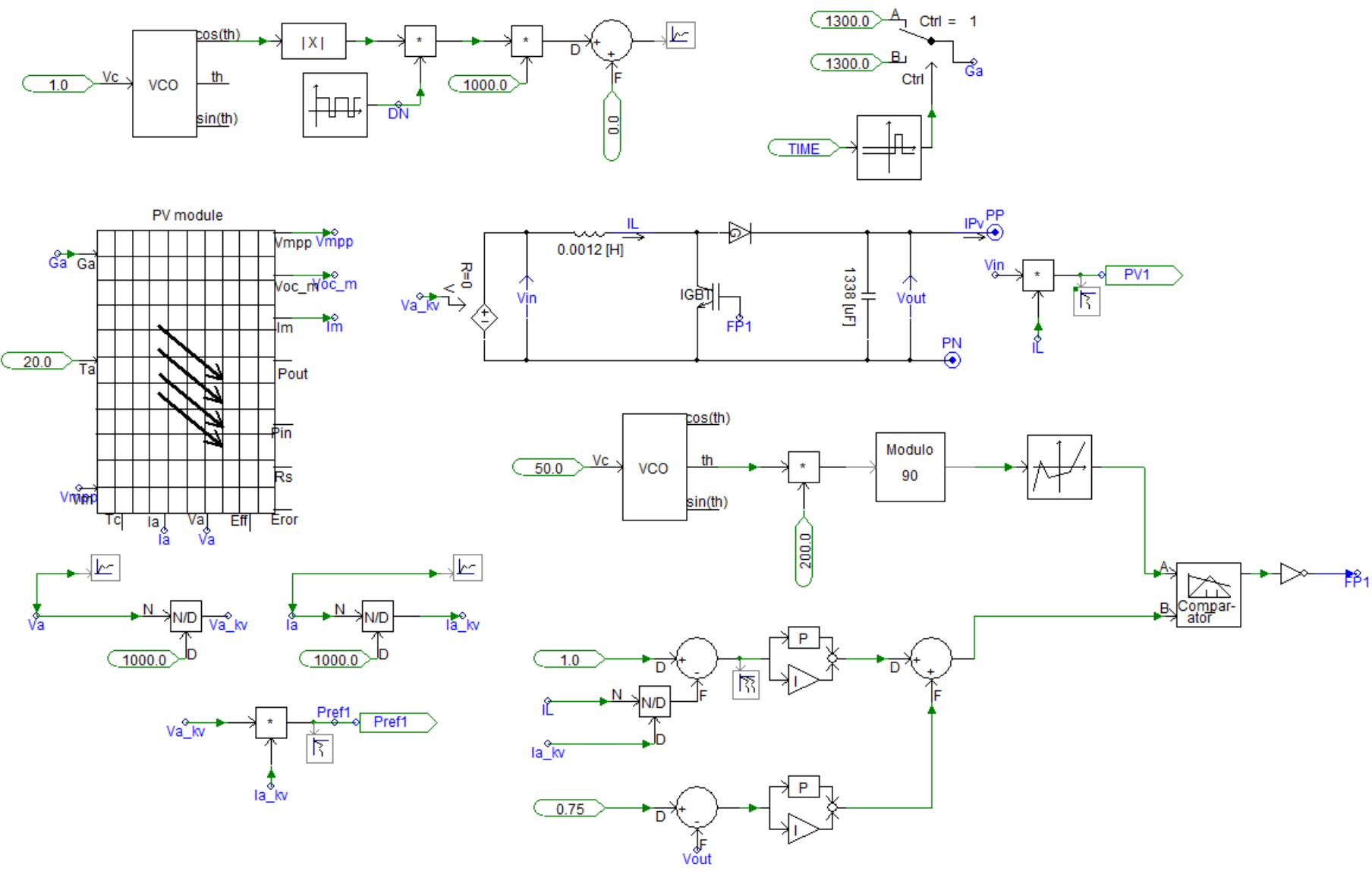

Figure 6.1: Modeling of $P V$ and $D C / D C$ 


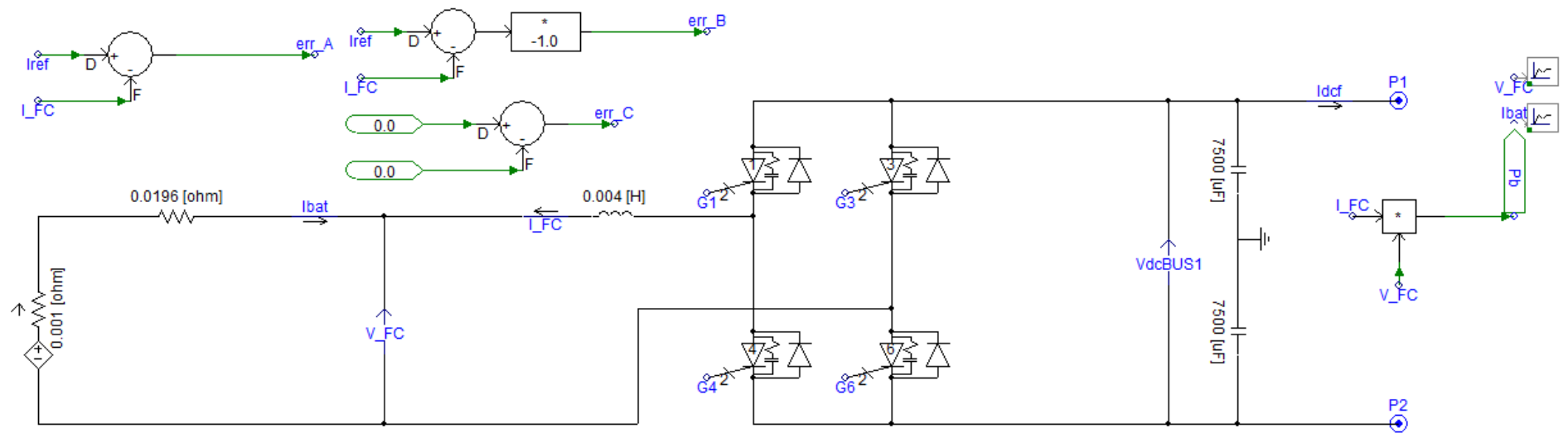

Figure 6.2: Battery Simulation Modeling
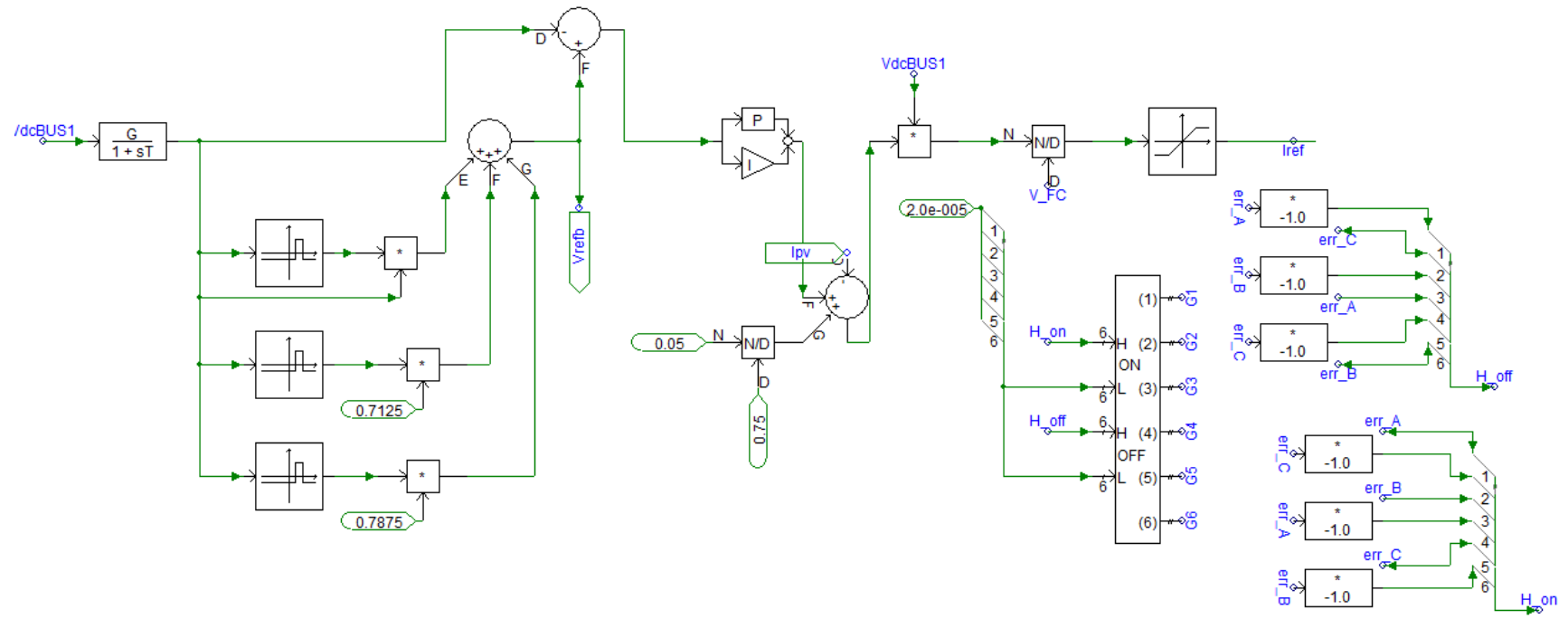

Figure 6.3: Battery Control System 


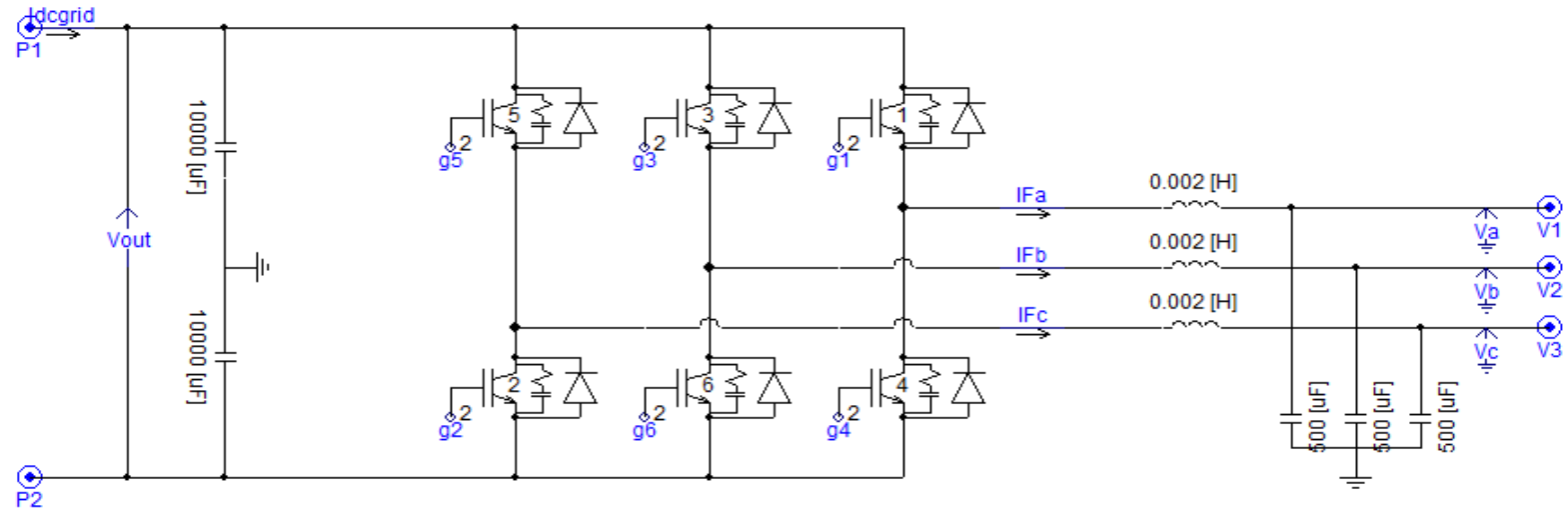

Figure 6.4: System's Inverter 

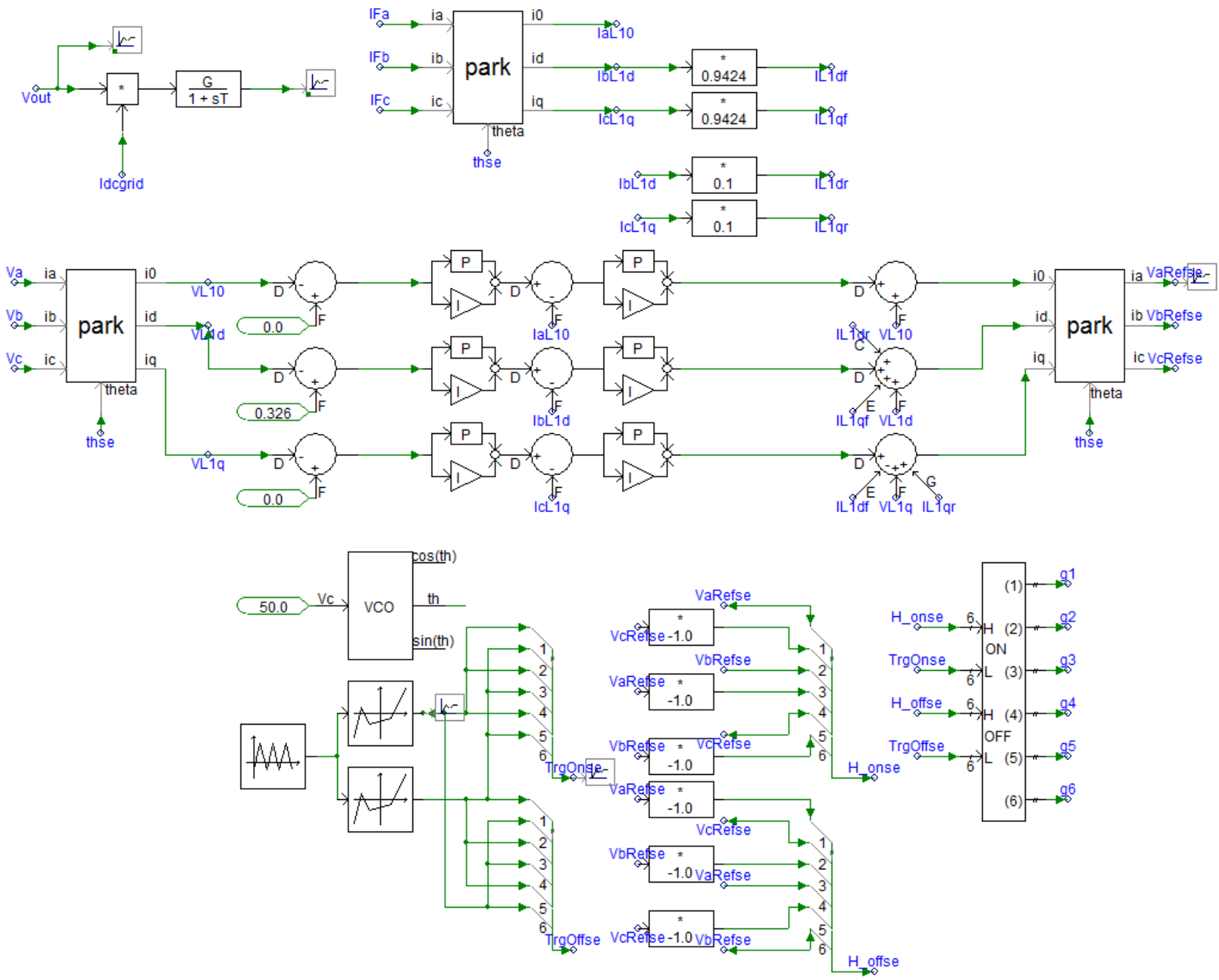

Figure 6.5: Control Strategy of Inverter

\subsection{Matab Modeling}

1. $\mathrm{ClC}$

2 .

3. $k=0.001$

4. $m p 1=9.4 e-5 ; m p 2=9.4 e-5 / 2 ; m p 3=9.4 e-5 / 2$;

5. $\mathrm{fS}=8000 ; \mathrm{Lf}=1.35 \mathrm{e}-3 ; \mathrm{nq}=1.3 \mathrm{e}-4 ; \mathrm{Cf}=50 \mathrm{e}-6 ; \mathrm{Kpv}=0.05 ; \mathrm{Kiv}=390 ; \mathrm{Kpc}=10.5 ;$ $\mathrm{KiC}=16000 ; \mathrm{F}=0.75 ; \mathrm{wC}=31.41 ; \mathrm{rC}=0.03 ; \mathrm{LC}=0.35 e-3 ; \mathrm{rf}=0.1 ; \mathrm{w} 0=314$;

6. $\mathrm{wn}=314 ; ; \circ \frac{0}{0} \div \circ \%$

7. $\mathrm{n}=3$;

8. ㅇverter one 111111111111111111111111111111111111111111

9. $\mathrm{mp}=\mathrm{mp} 1$;

10. $\quad$ Vod=380.8;

11. Voq=0;

12. Iod=11.4;

13. Ioq=0.4;

14. $\quad$ Ild $=11.4$;

15. Ilq $=-5.5$;

16. $\operatorname{delta} 0=0$; 


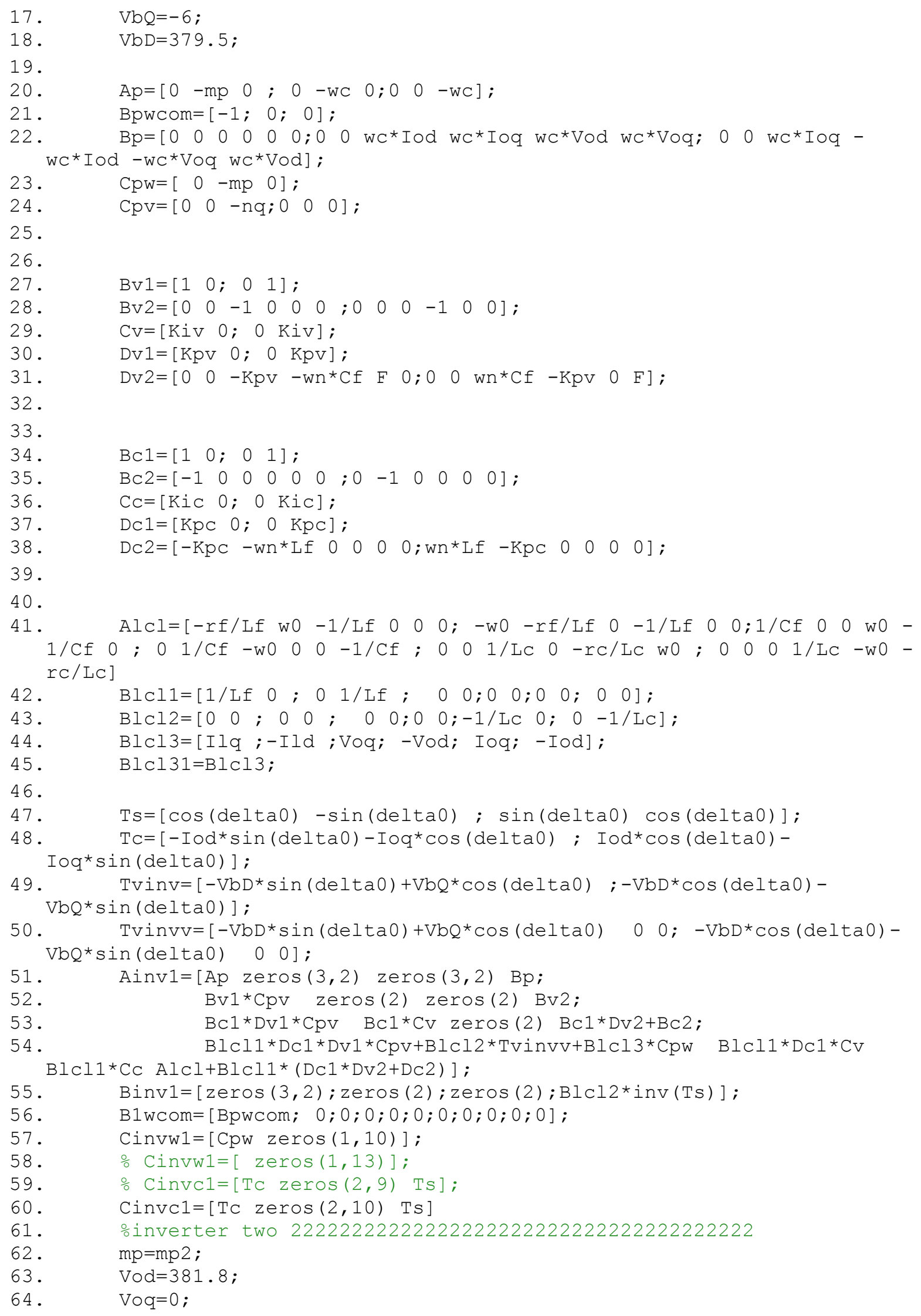




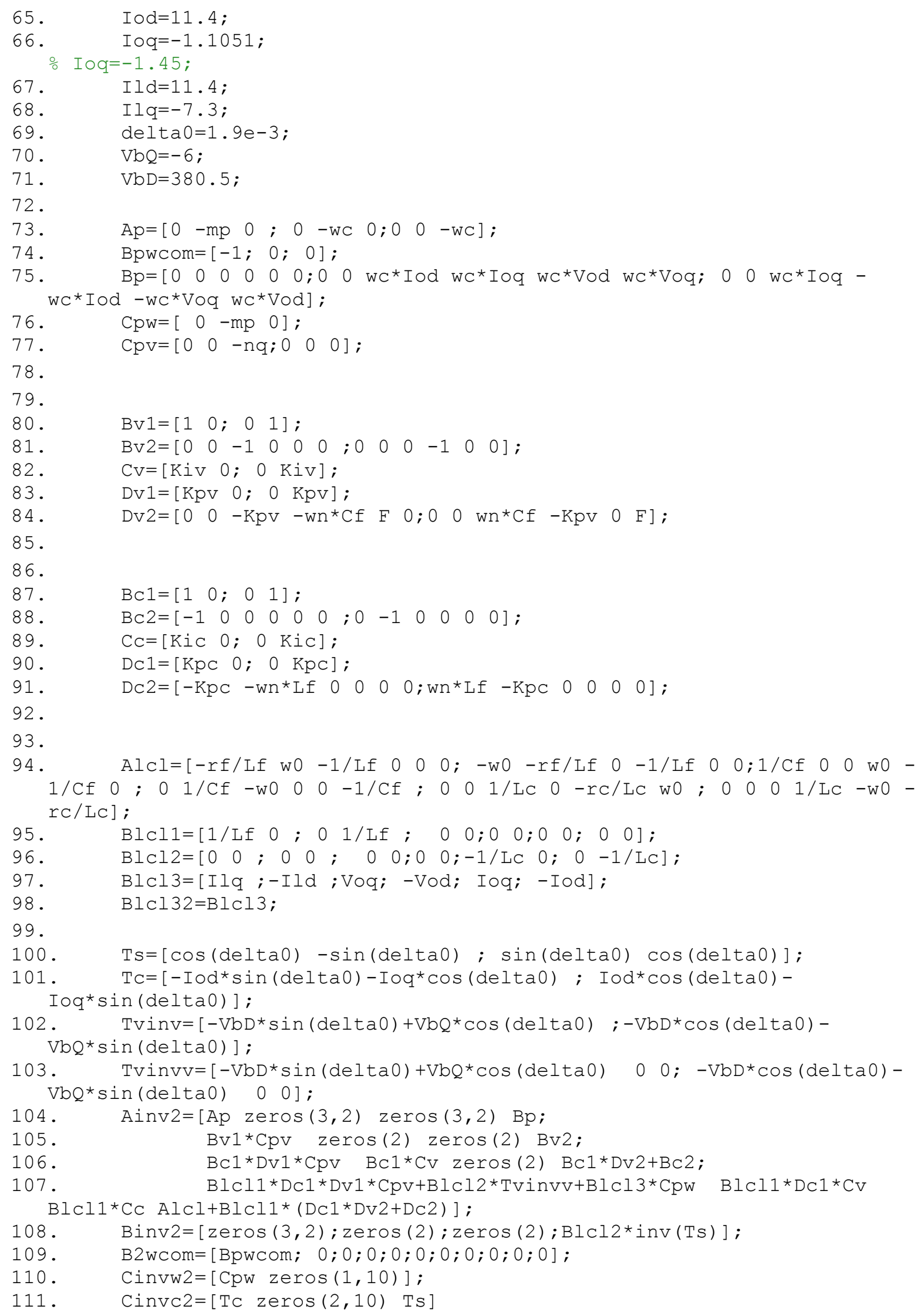




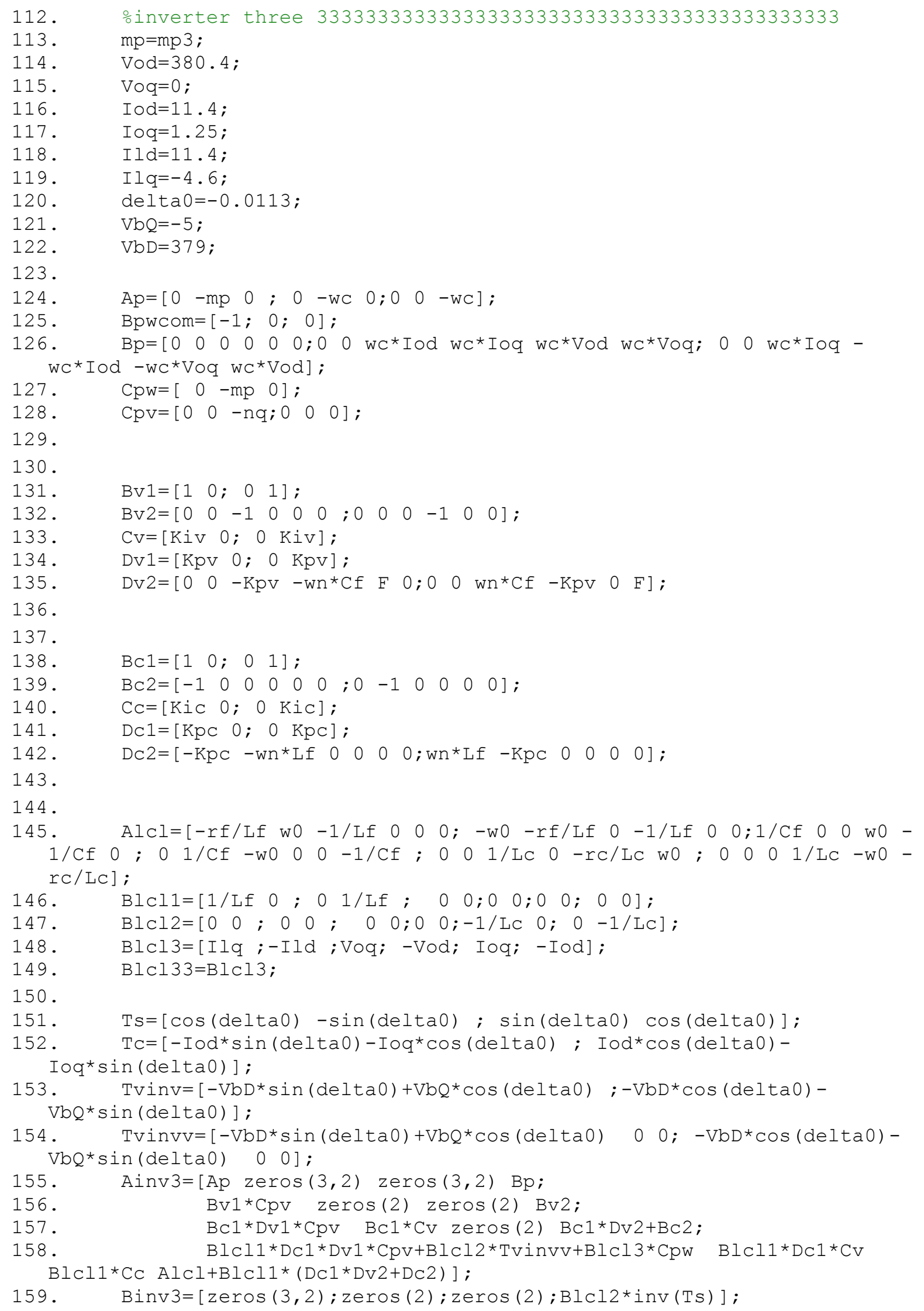




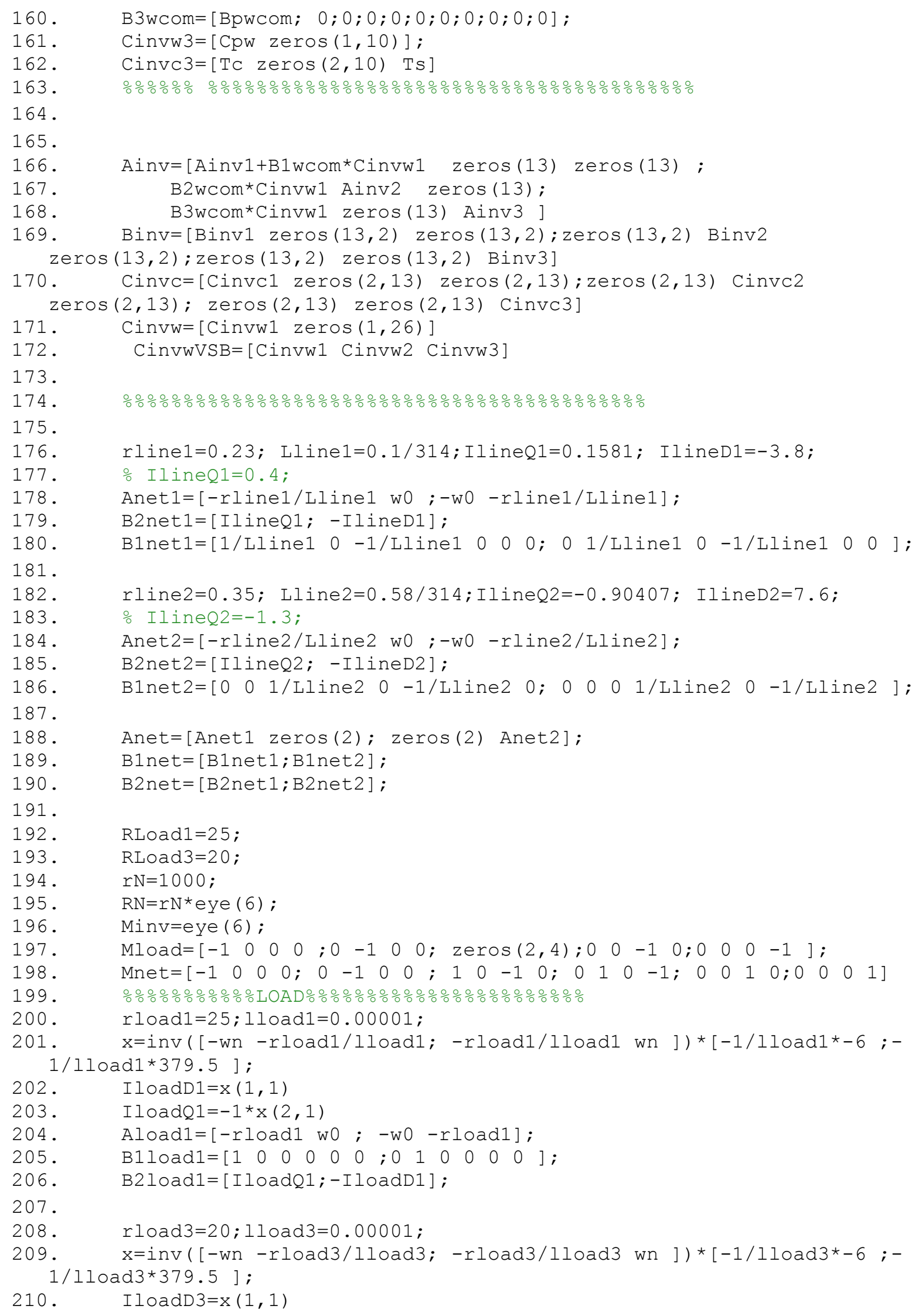




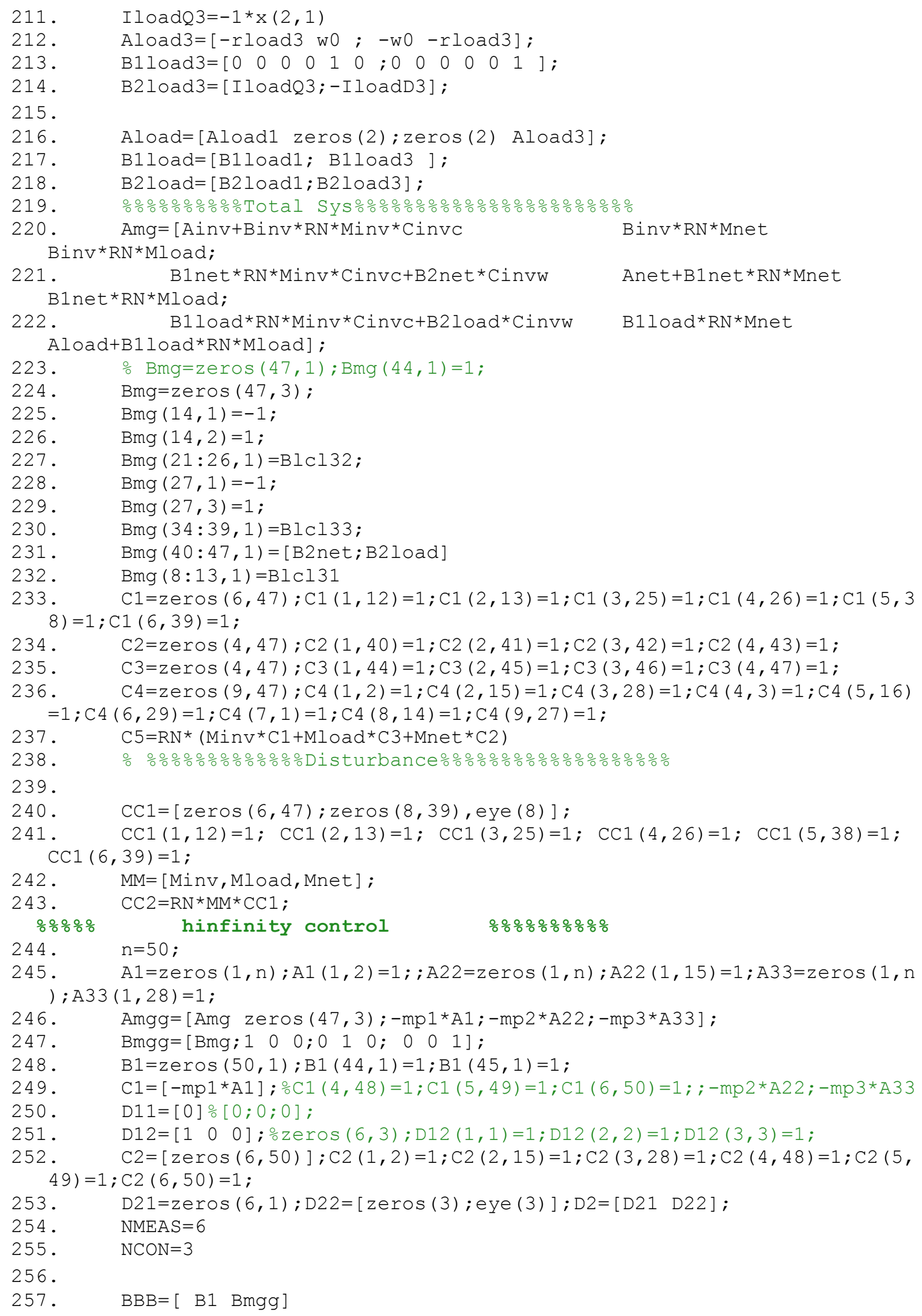


258. $\quad \mathrm{CCC}=[\mathrm{C} 1 ; \mathrm{C} 2]$

259. $\mathrm{DDD}=\left[\begin{array}{ll}\mathrm{D} 11 & \mathrm{D} 12 ; \mathrm{D} 2\end{array}\right]$

260. $\mathrm{P} 3=\mathrm{sS}(\mathrm{Amgg}, \mathrm{BBB}, \mathrm{CCC}, \mathrm{DDD})$ 


\section{CONCLUSION}

In this thesis, a comprehensive state space model of a microgrid consisting of PQ and VSI inverters is presented. The effect of changing the nominal frequency set-point for VSI inverters and active and reactive power set-points for PQ inverters is taken into account all through the modeling which is then used to investigate the small signal stability and the effect of proposed secondary control. An 11 bus microgrid is used to illustrate the effectiveness of the proposed approach. The stability results presented at chapter 4 indicate that the modeling method can be used to analyze the stability of the microgrid primary control. In addition, the simulation results show the secondary controller successfully coordinates the multiple source types under challenging load conditions while managing their individual constraints. In chapter 5, a consensus algorithm is used for the secondary voltage control of an islanded microgrid. The chosen control is totally distributed, applied on a network of agents which has been optimally designed based on the required connectivity. After defining the best communication network and the proper updating matrix for the agents, the ACA is used as the decision-making algorithm. The main purpose of the ACA is to keep the average voltage of all pilot buses at a desired level during islanding. Consequently, not only the DG buses are providing power at proper voltage but also the loads are being fed at the appropriate voltage level. This algorithm along with the primary control is simulated on a system with 3 pilot nodes in MATLAB/ Simulink and the results guarantee proper voltage stability as well as voltage quality by establishing consensus and coordination between the distributed agents. Finally, at the end of chapter 5, a secondary cooperative voltage control has been proposed in which both VSI and PQ inverters will work together to alleviate any load change in the system. This cooperative method is based on the zone dedication which is calculated based on the sensitivity analysis to assign specific loads to each DG in the system. The above method has been applied to a microgrid with 13 buses and 4 DGs. The simulation result in this chapter shows the effectiveness of the algorithm in case of load changes in the system. 


\section{FUTURE WORK}

In this research, comprehensive modular state-space modeling, control development and management strategies of microgrids containing inverter-based distributed energy resources are studied, in which small signal stability enhancement for small-scale power systems is achieved and a novel decentralized secondary frequency and voltage control for microgrids are introduced by decomposing the system into virtual control areas. Working on specifications of DERs itself and its application when integrated in the microgrids are inevitably attached to each other and a research could be successful which follows both of them simultaneously. Also, employing advanced optimization methods, signal processing techniques (such as reduced-order models which manage system complexity, and system identification methods which determine system dynamics), and non-classical control methods (such as model predictive control and game theoretic control which address emerging operational theories of the power system) can be considered as a future development.

During my research, I have developed a simple, yet elaborate distributed control strategy for the secondary level control. I conceptualized a distributed framework for the microgrid control as the building block of the future power system. I developed a novel secondary control to devise set points that ensure feasibility of the microgrid operation subsequent to a major change or disturbance. The aforementioned novel concept replace a new vision for the microgrid's control with the common solution of considering the whole microgrid as one united electric power system in which all the micro sources have the same contribution based on their participation factors. In fact, the novel approach, tries to improve the transient response of system by dividing the microgrid to multi area systems and using an artificial tie-line power error between them as a new term in Area Control Error (ACE) named Virtual Area Control Error (VACE). More than improving the transient response, there are also other reasons, which make this research necessary to be pursued. By deregulation in distribution power market, the priority of micro sources for feeding loads will vary. So, as a result of this developing deregulation in distribution power market, the investigation of MultiArea-Microgrid (MAM) seems important. This formation will make deregulation much more applicable by improving the performance, economical and reliability aspects. It also makes the communication more practical by limiting the required communication links. Even though I have worked on these approaches during my $\mathrm{PhD}$, still lots of research activities are required to consider each and every aspect in details and make the Multi-Area-Microgrid idea possible for any further technical implementation.

\section{Probable future works:}

$\checkmark$ Improving the microgrid's intelligent operation by integrating smart devices into the system and developing smart grids by utilizing communication-based multi-agent systems 
$\checkmark$ Different computational approaches can be employed in order to create intelligent agents for power system operations

$\checkmark$ Developing programming tools for modeling and analysis of the electric system which can visualize the theoretical concept of smartgrids control and implementation

$\checkmark$ Adding the state equations of other component such as synchronous machines.

$\checkmark$ Analyzing the stability of the microgrid in case of having fault in the system.

$\checkmark$ Considering the DC bus properties instead of assuming ideal situation for it.

$\checkmark$ Considering the switching effect of inverter on the modeling in order to have more accurate results.

$\checkmark$ Analyzing the systems which are not balanced.

$\checkmark$ Using learning algorithm to improve the voltage and frequency control approaches.

$\checkmark$ Considering min and max consensus for voltage regulation. 


\section{REFERENCES}

[1] Y. Riffonneau, S. Bacha, F. Barruel, A. Delaille, "Energy flow management in grid-connected PV systems with storage - A deterministic approach", IEEE International Conference on Industrial Technology, pp. 1-6, 2009.

[2] F. Doost Mohammadi, A. Feliachi, "Adaptive Price Management in Hybrid Microgrid in Presence of PV and Battery Energy Storage System”, IEEE PES T\&D Conference, Chicago, IL, April 14-18, 2014.

[3] H. Keshtkar, J. Solanki, S.K. Solanki, "Application of PHEV in load frequency problem of a hybrid microgrid", North American Power Symposium (NAPS), 2012, IEEE, pp. 1-6.

[4] F. Doost Mohammadi, M. Ghorbani, A. Feliachi, M.A. Choudhry "Novel Load Frequency Control Approach Based on Virtual Area Error in a Microgrid including PV and Battery Energy", IEEE PES General Meeting, Washington, D.C., July 26-29, 2014.

[5] M. C. Chandorkar, D. M. Divan and R. Adapa "Control of parallel connected inverters in standalone AC supply systems", IEEE Trans.Ind. Appl., vol. 29, pp.136-143 1993

[6] F. Katiraei and M. R. Iravani "Power management strategies for a microgrid with multiple distributed generation units", IEEE Trans. Power Syst., vol. 21, pp.1821 -1831 2005

[7] A. Feliachi, "On the Control of Re-Structured Electric Power systems," International Journal of Control, Automation, and Systems, Special Issue on Recent advances in Power System Control, Vol. 3, No. 2, pp. 363$375,2005$.

[8] M. Amin, "Powering the 21st Century," IEEE Power \& Energy, pp. 93-96, March-April, 2005.

[9] A.P. Lopes, C. L. Moreira, A. G. Madureira, "Defining control strategies for MicroGrids islanded operation", IEEE Transactions on Power Systems, vol.21, no.2, May 2006.

[10] S. Ahn, J.Choi, "Power Sharing and Frequency Control of an Autonomous Microgrid Considering the Dynamic Characteristics of Distributed Generations", Journal of International Council on Electrical Engineering, vol. 2, no. 1, pp. 39 44, 2012.

[11]Z. Jiang, X. Yu, "Active power - Voltage control scheme for islanding operation of inverterinterfaced microgrids" Power \& Energy Society General Meeting, PES '09, pp. 1-7, 2009.

[12] T. Ota, K. Yukita, H. Nakano, Y. Goto, K. Ichiyanagi, "Study of load frequency control for a microgrid", Australasian Universities Power Engineering Conference, AUPEC 2007, pp. 1-6, Dec. 2007.

[13] K. Sedghisigarchi, A. Feliachi, "Impact of fuel cells on load-frequency control in power distribution systems", IEEE Trans on Energy Conversion, vol. 21, no. 1, March 2006.

[14] A. Dimeas and N. D. Hatziagyriou, "A multiagent system for microgrids, " in Proc. IEEE PES General Meeting, Denver, CO , Jun. 2004, PESGM2004-001244.

[15] A. Dimeas and N. D. Hatziagyriou, "A MAS architecture for microgrids control," in Proc. 2005, The 13th International Conference on Intelligent Systems Application to Power Systems, pp. 402-405.

[16] A. Dimeas and N. D. Hatziargyriou, "Operation of a multiagent system for microgrid control", IEEE Trans on Power System, vol. 20, no. 3, Aug. 2005, pp. 1447-1455.

[17] A. Dimeas and N. D. Hatziagyriou, "Agent based control for microgrids,"2007, IEEE Power Engineering Society General Meeting. 
[18] J. M. Guerrero, J. C. Vasquez, J. Matas, L. G. de Vicua and M. Castilla, "Hierarchical control of droop-controlled AC and DC microgrids: A general approach toward standardization," IEEE Trans. on Industrial Electronics, vol. 58, no. 1, pp. 158-172, Jan. 2011.

[19] W. Liu, W. Gu, "Decentralized Multi-Agent system-Based cooperative Frequency Control for Autonomous Microgrids with Communication Constraints" IEEE Transaction on Sustainable Energy, April 2014.

[20] A. Bidram, A. Davoudi, F. L. Lewis, J.M.Guerrero, "Distributed Cooperative Secondary Control of Microgrids Using Feedback Linearization" IEEE Transaction on Power system, August 2013.

[21] W. Gu, W. Liu, Z.Wu, B. Zhao, W, Chen "Cooperative Control to Enhance the Frequency Stability of Islanded Microgrid with DFIG-SMES ” Journal Energies, April 2013.

[22] P. Massioni and M. Verhaegen, "Distributed control for identical dynamically coupled systems: A decomposition approach, IEEE Trans. On Automatic Control, vol. 54, no. 1, pp. 124-135, Jan. 2009.

[23] N. Pogaku, M. Prodanovic T. C. Green, "Modeling, Analysis and Testing of Autonomous Operation of an Inverter-based Microgrid" IEEE Transaction on Power Electronics, March 2007.

[24] Mohamed, Y.A.-R.I.; El-Saadany, E.F., "Adaptive Decentralized Droop Controller to Preserve Power Sharing Stability of Paralleled Inverters in Distributed Generation Microgrids," in Power Electronics, IEEE Transactions on , vol.23, no.6, pp.2806-2816, Nov. 2008

[25] Bidram, A.; Davoudi, A.; Lewis, F.L., "A Multiobjective Distributed Control Framework for Islanded AC Microgrids," in Industrial Informatics, IEEE Transactions on, vol.10, no.3, pp.1785-1798, Aug. 2014

[26] Schiffer, J.; Seel, T.; Raisch, J.; Sezi, T., "Voltage Stability and Reactive Power Sharing in Inverter-Based Microgrids With Consensus-Based Distributed Voltage Control," in Control Systems Technology, IEEE Transactions on , vol.PP, no.99, pp.1-1

[27] G. D'ýaz, C. Gonz'alez-Mor'an, J. G'omez-Aleixandre and A. Diez, "Complex-valued state matrices for simple representation of large autonomous microgrids supplied by PQ and Vf generation," IEEE Trans. on Power Systems, vol. 24, no. 4, pp. 1720-1730, Nov. 2009.

[28] F. Katiraei, M. R. Iravani, and P. W. Lehn, "Small-signal dynamic model of a micro-grid including conventional and electronically interfaced distributed resources," IET Gen., Transm., Distrib, 2007.

[29] W. Zheng, J. Cai, "A multi-agent system for distributed energy resources control in microgrid", 2010 5th International Conference on Critical Infrastructure (CRIS), pp. 1-5, Sept. 2010.

[30] C. Min and G. A. Rincon-Mora, "Accurate electrical battery model capable of predicting runtime and I-V performance," IEEE Transactions on Energy Conversion, vol. 21, pp. 504-511, 2006.

[31] Mathworks. (2012). Battery. Available at :

http://www.mathworks.com/help/toolbox/physmod/powersys/ref/battery.html

[32] A. Keyhani, "Design of Smart Power Grid Renewable Energy Systems,” Wiley 2011

[33] Mathworks. (2012) Simplified Synchronous Machine. Available at: http://www.mathworks.com/help/toolbox/physmod/powersys/ref/simplifiedsynchronousmachine.html

[34] Polycrystalline PV Module 60 cells. Available at http://www.upsolar.com/usa/products/monocrystalline. aspx $?$ type=4\#135 
[35] K. Ishaque, Z. Salam, and H. Taheri, “Accurate MATLAB Simulink PV System Simulator Based on a Two-diode Model," Journal of Power Electronics, In Press, Corrected Proof

[36] T. Esram and P. L. Chapman, "Comparison of Photovoltaic Array Maximum Power Point Tracking Technique," IEEE Transaction on Energy Conversion, Vol. 22, pp. 439-449, 2007.

[37] Vieira, J.A.B., Mota, A.M., "Implementation of a stand-alone photovoltaic lighting system with MPPT battery charging and LED current control", Proc. IEEE International Conference on Control Applications, pp. 185 190, Pacifico Yokohama, Yokohama, Japan, Sept. 8-10, 2010.

[38] Olfati-Saber, R.; Murray, R.M., "Consensus problems in networks of agents with switching topology and timedelays," in Automatic Control, IEEE Transactions on, vol.49, no.9, pp.1520-1533, Sept. 2004

[39] D. Rerkpreedapong, "Novel Control Design and Strategy for Load Frequency Control in Restructured Power Systems" PhD Dissertation at West Virginia University

[40] M. Fiedler, "Algebraic connectivity of graphs," Czech. Math. J., vol. 23, no. 98, pp. 298-305, 1973.

[41] Y. Xu and W. Liu, "Novel Multiagent Based Load Restoration Algorithm for Microgrids," in IEEE Transactions on Smart Grid, vol. 2, no. 1, pp. 152-161, March 2011.

[42] N. E. Leonard and A. Olshevsky, "Cooperative learning in multi-agent systems from intermittent measurements," 2nd IEEE Conference on Decision and Control, Firenze, 2013, pp. 7492-7497.

[43]D. K. Khatod, V. Pant and J. Sharma, "A novel approach for sensitivity calculations in the radial distribution system," in IEEE Transactions on Power Delivery, vol. 21, no. 4, pp. 2048-2057, Oct. 